\title{
Genetic factors associated with variation in abundance of the invasive yellow crazy ant (Anoplolepis gracilipes)
}

Monica Alexandra Maria Gruber

\author{
A thesis submitted to \\ Victoria University of Wellington \\ in fulfilment of the requirements for the degree of \\ Doctor of Philosophy \\ in Ecology and Biodiversity
}

Victoria University of Wellington

Te Whare Wānanga o te Ūpoko o te Ika a Māui 

This thesis was conducted under the supervision of

Associate Professor Phil J. Lester (primary supervisor)

Victoria University of Wellington

Wellington, New Zealand

and

Dr. Benjamin D. Hoffmann

Ecosystem Sciences, Tropical Ecosystems Research Centre

Winnellie, Northern Territory, Australia

and

Dr. Peter A. Ritchie

Victoria University of Wellington

Wellington, New Zealand 



\section{Abstract}

A key component of successful invasion is the ability of an introduced population to reach sufficient abundance to persist, spread, and alter or dominate the recipient biological community. Genetic diversity is one of many factors that may contribute to population dynamics, but has important ramifications for biological fitness, and thus invasion success in the long term. I explored genetic factors associated with variation in abundance (i.e., differential invasion success) of the yellow crazy ant Anoplolepis gracilipes in the Indo-Pacific region, primarily focussing on Arnhem Land in Australia's Northern Territory.

I explored five aspects that I hypothesised could contribute to variation in the abundance of this ant: 1) I investigated the unusual reproductive mode of $A$. gracilipes, and tested whether it involved dependent-lineage genetic caste determination (DL GCD) in Arnhem Land. In DL GCD systems populations require hybridisation between genetically distinct groups to produce both reproductive and worker castes. Asymmetry in the ratio of different lineages may result in low abundance and population collapse. I found no evidence for a DL GCD system in A. gracilipes, and thus its abundance in Arnhem Land does not appear to be constrained by any lineage ratio asymmetry. Worker reproduction (either of males or asexual production of other workers) also appeared unlikely. The reproductive mode of the species remains fascinating but enigmatic; 2) I explored whether multiple source populations were responsible for the observed variation in abundance in Arnhem Land (i.e., is abundance associated with propagule pressure, or populations from different sources), and if the population has diverged since introduction. The A. gracilipes population in Arnhem Land stemmed from a single source, and thus propagule pressure was apparently not responsible for variation in abundance. In contrast to many invasive ants, population divergence has occurred since introduction; 3) I tested the hypotheses that genetic variation was associated with variation in abundance in Arnhem Land, and that ecological success was density-dependent. While the population divergence found in Chapter 3 was not related to variation in abundance, genotypic diversity was higher in more abundant nest clusters. These more abundant nest clusters were in turn associated with lower native ant species diversity, and a difference in 


\section{Abstract}

composition of the invaded ant community (i.e., greater ecological success); 4) I revisited the invasion of the yellow crazy ant in Tokelau to determine whether a haplotype that was linked to greater abundance and dominance of the ant community has increased in distribution. Although ants of the inferred dominant haplotype were implicated in most new invasions, their abundance was substantially lower than previously observed in Tokelau; 5) I conducted a preliminary analysis of the metagenomic diversity of $A$. gracilipes endogenous parasites and symbionts among populations from Christmas Island, Okinawa, Samoa and Arnhem Land. Bacterial community composition and diversity differed between the study populations. Variation in abundance among $A$. gracilipes populations in Arnhem Land was not due to parasite load on populations with low abundance. However, low abundance of A. gracilipes was correlated with lower microbial diversity overall, and higher prevalence of some groups, notably two that confer antibiotic properties.

Together, my findings suggest that propagule pressure, reproductive mode and haplotype-specific effects do not appear to be associated with variation in A. gracilipes abundance. Other genetic factors I investigated do appear to be associated with the variation in A. gracilipes abundance and effects on the invaded ant communities. Genotypic diversity was positively related to the abundance of A. gracilipes in Arnhem Land, and this relationship may be affected by population divergence through population bottlenecks. In addition, differences in bacterial diversity among populations highlighted several candidate bacteria that could be associated with variation in abundance, which would be a topic of future work. Although genetic factors are often implicated in the successful establishment of invasive species, my thesis demonstrates that genetic factors may also be associated with post-establishment population dynamics. 


\section{Acknowledgements}

I have been very fortunate to have had the most extraordinary supervisor, Phil Lester. Phil's good humour, sensible advice, support and willingness to send me to places he would rather go is truly appreciated beyond words. Everyone should be so lucky to have a supervisor like Phil!

The phenomenal work ethic that Ben Hoffmann demonstrates inspires me with awe. I benefitted greatly from Ben's positive attitude, tremendous capacity for field work, and knowledge of ant ecology. Without Ben's work, this project would have been impossible.

Pete Ritchie has mentored and encouraged me since I was an undergraduate, for which I am very grateful. Pete's inspiring second, third and fourth year courses in population genetics and evolution initiated my interest in the synthesis between population genetics, evolution and ecology.

Many people provided assistance with data collection. In Australia, Alan Andersen's expert ant identification skills were indispensable. Thanks to Ben Hoffmann and Daryl Lacey for help with pitfall trapping in Arnhem Land, and Claire Tailly for help sorting the traps in Darwin. Many thanks also to Ben and Debbie Hoffmann for their hospitality in Humpty Doo. Harvey, Maya and Samuel Buchman were great field assistants in Samoa. Chris Boland and his Parks Australia North team provided awesome hospitality and field assistance on Christmas Island. My Tokelau work was only possible thanks to Ray Pierce. Many thanks also to Allan Burne for helping with collections in Tokelau, haplotyping, and ant identifications. Thanks to Ben Hoffmann for samples from Christmas Island, Kazuki Tsuji and Hiroyuki Shimoji for samples from Okinawa, and Phil Lester for historical samples from Tonga and Tokelau. 


\section{Acknowledgements}

I am grateful to my collaborators and others who made suggestions that improved my critical thinking, analysis and writing. In particular I thank my supervisors for their patience and many insightful suggestions. Many discussions with Sébastien Rioux Paquette enhanced my understanding of population genetics. Early drafts of Chapter 2 benefitted from comments by Theo Evans, Joe Zuccarello, Julien Grangier, Alexandra Sebastien, Allan Burne, Claudie Doums, Kazuki Tsuji and Christian Peeters. Discussions with Allan Burne, Elizabeth Heeg and Sébastien Rioux Paquette, and suggestions from Julien Grangier and Jochen Drescher improved Chapter 3. Comments by anonymous editors and reviewers on drafts of Chapters 2, 3, 4 and 5 provided critical insights that improved these chapters. My examiners Rob Cruickshank, Andy Suarez and Joe Zuccarello also provided comments and suggestions that improved the thesis and manuscripts. Thanks to Allan Burne and Harvey Buchman for proofreading the thesis.

Chapter 6 would not have been possible without high performance computing, and I am grateful to Pete Ritchie, Cameron Jack, John Hine, and particularly Kevin Buckley for facilitating this. Thanks to Cameron Jack for writing the SolexaStats java package. Anna Friedlander wrote the taxon4blast.pl Perl script, which saved me much time, and I greatly appreciated her enthusiasm for the project. Sincere thanks also to the University of Auckland's Nick Jones, Richard Hosking and Sina Masoud-Ansari for facilitating the Summer of eResearch project that enabled Anna's contribution. Macrogen's Ryan Jeong and Jong-so Kim conducted Illumina sequencing, and Illumina's Karl Sluis and Derek Campbell supplied planning advice. Lesley Collins' Perl workshop and the Massey University bioinformatics course run by Lesley and Patrick Biggs were very useful introductions to bioinformatics for next generation sequencing.

I also would like to acknowledge Nicky Nelson, whose support through Allan Wilson Centre studentships enabled me to gain valuable basic skills in genetic analysis and advanced statistics as an undergraduate. Through this I was also very fortunate to have been introduced to $\mathrm{R}$ and generalised linear models by Shirley Pledger. Thanks also to Murray Williamson for a free GIS introduction course that proved tremendously useful. 
Thanks to Alan Hoverd and Lesley Thompson and their teams for the many things they do that facilitate research in SBS. Neville Higgison, Adrian Pike, Mary Murray, Sandra Taylor, Patricia Stein, Delwyn Carter-Jarratt and Paul Marsden were particularly helpful. Barry Lewis of the Scholarships Office was always prompt and helpful in resolving my queries.

My project was facilitated by the financial and / or logistic support of the VUW Research Trust, CSIRO Australia, Parks Australia North, Rio Tinto Alcan, Dhimurru Aboriginal Corporation, Conservation International and the Government of Tokelau. All field and laboratory work complied with all the permit requirements, ethical laws and guidelines of the countries and landowners involved. I am very grateful for the financial support of a VUW PhD Scholarship, VUW Hunter Scholarship, TEC Top Achiever Doctoral Scholarship and NZ Postgraduate Study Abroad Award.

Most importantly, my eternal gratitude goes to my lovely family - Harvey, Maya and Samuel Buchman - who coped with my numerous physical absences, mental distractions and bewildering tales of ants, genes, and the joys and frustrations of postgraduate study. I couldn't (and probably wouldn't) have done it without you. I'm also extremely grateful for the support of my mother Dorothea Gruber and mother-in-law Miriam Buchman. I wish that my late father Matthaeus Gruber, and my dear uncle Claude Hellberg, who were such positive influences on my early life, could also have been here to share this journey. 


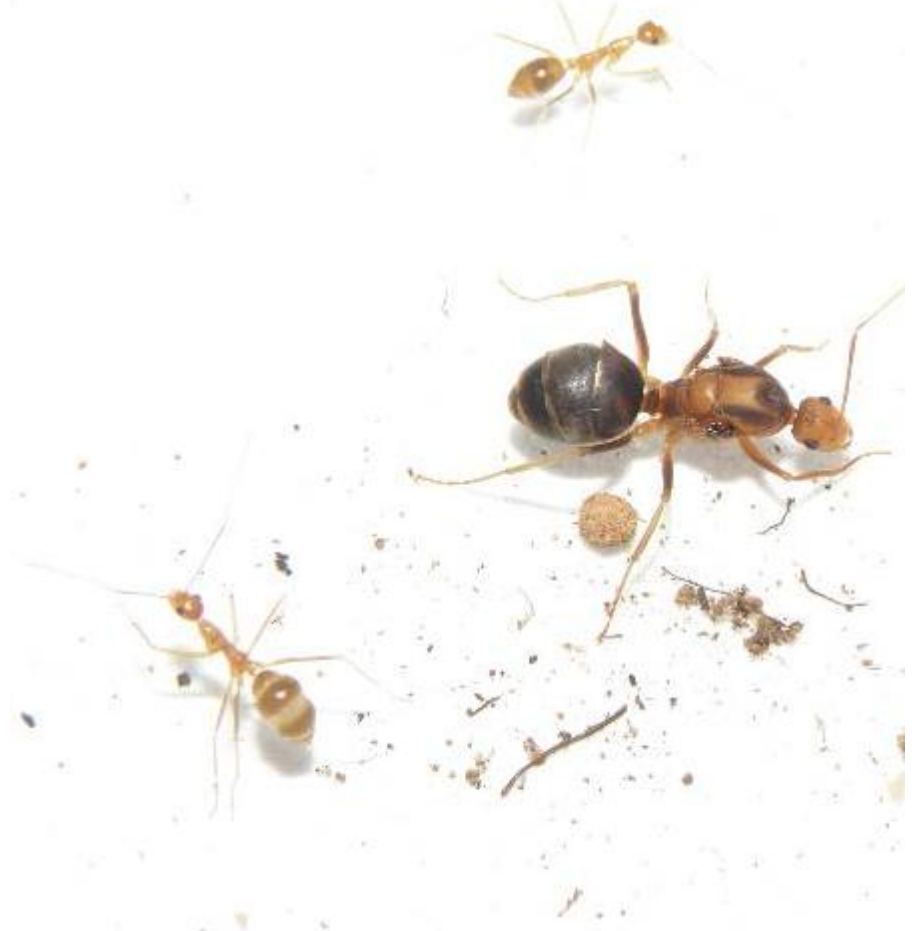




\section{Contents}

Abstract

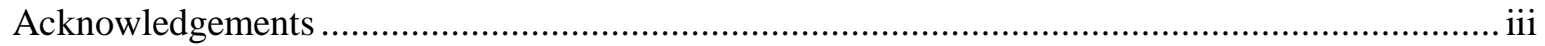

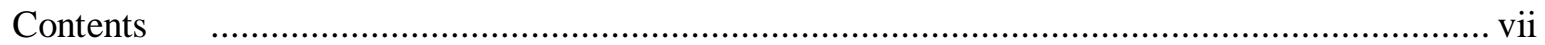

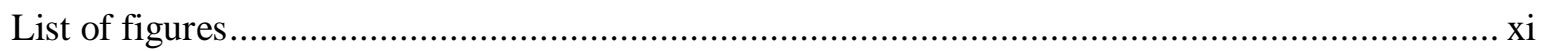

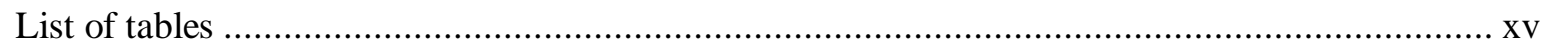

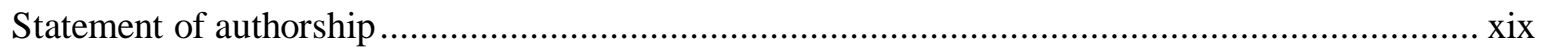

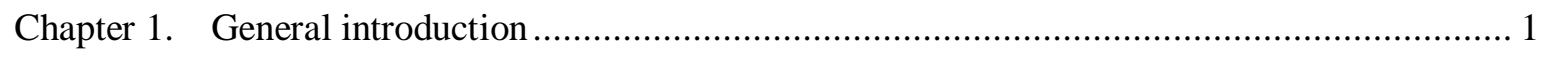

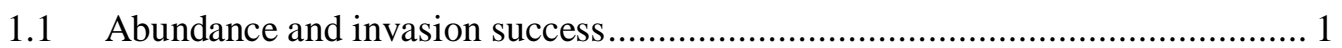

1.2 Genetic factors and the success of invasive ants ......................................... 3

1.3 Ecological factors also contribute to the invasion success of ants .................... 8

1.4 Focal study system: Anoplolepis gracilipes in Arnhem Land ......................... 10

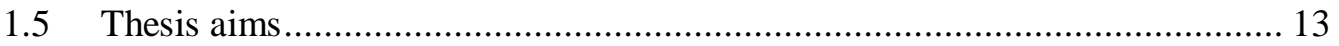

Chapter 2. The conundrum of the Anoplolepis gracilipes reproductive mode: no evidence for dependent lineage genetic caste determination ..................................................... 15

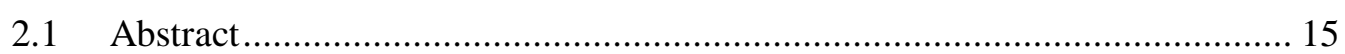

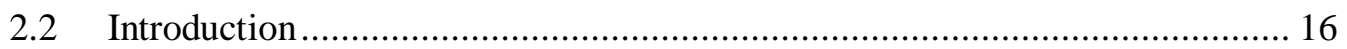

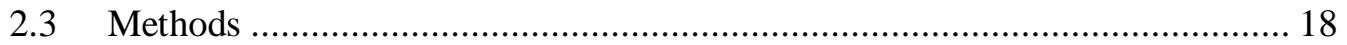

2.3.1 Study site and sampling …..................................................... 18

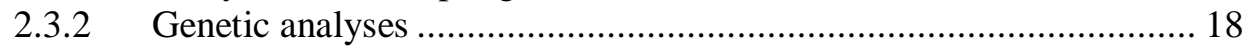

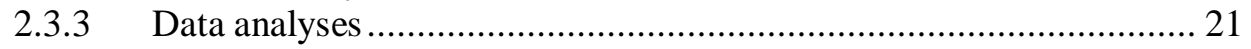

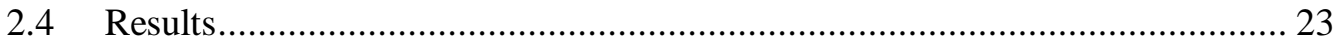

2.4.1 Population genetic parameters...................................................... 23

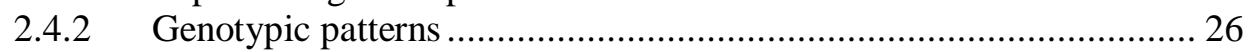

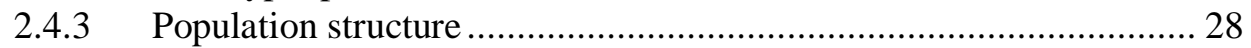

2.4.4 Worker reproduction........................................................................ 30

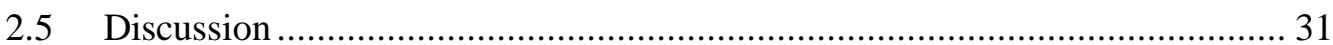

2.5.1 Dependent lineage genetic caste determination............................. 31

2.5.2 Potential alternative reproductive modes ........................................ 32

2.5.3 The enigma of diploid males .......................................................... 34

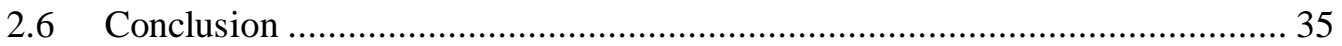




\section{Contents}

Chapter 3. Recent behavioural and population genetic divergence of an invasive ant in a novel environment

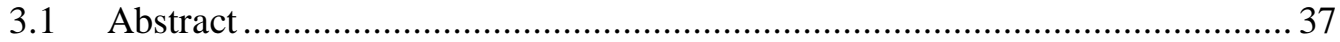

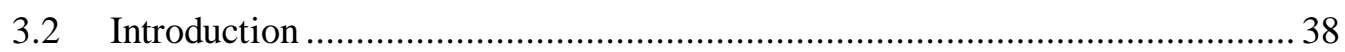

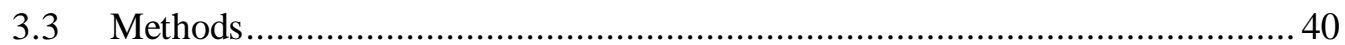

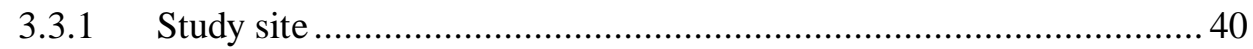

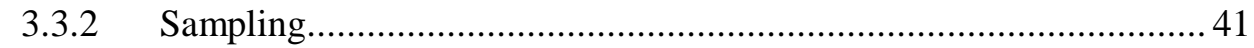

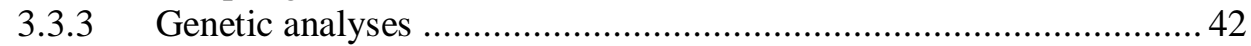

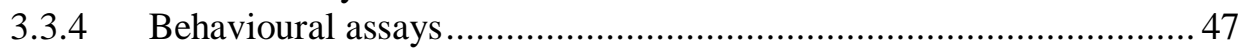

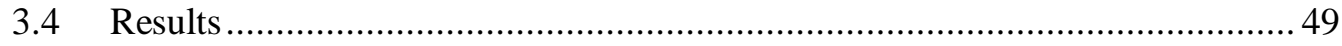

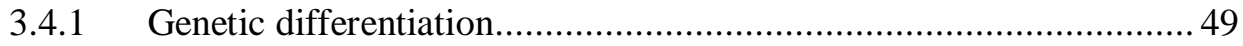

3.4.2 Genetic diversity ..................................................................... 51

3.4.3 Behavioural differentiation in relation to population genetic structure...

3.4.4 Abundance in relation to population genetic structure ..........................5 54

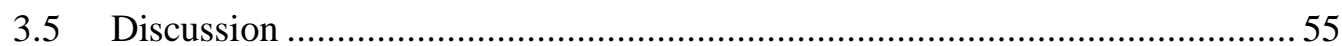

3.5.1 The relationship between population structure and abundance ..........55

3.5.2 Divergence of Anoplolepis gracilipes in Arnhem Land .................... 56

3.5.3 Nestmate recognition and aggression................................................5 57

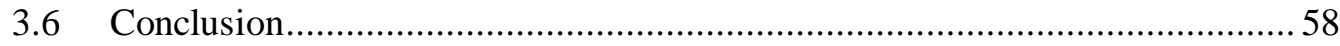

Chapter 4. Higher genetic diversity is associated with fine-scale momentary abundance of an

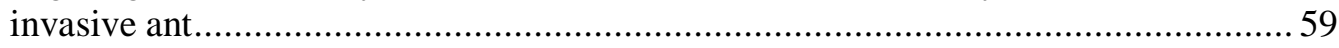

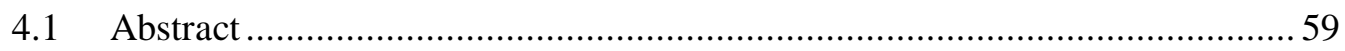

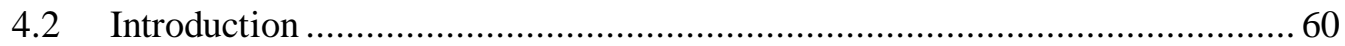

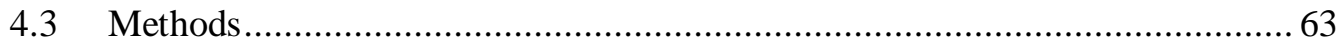

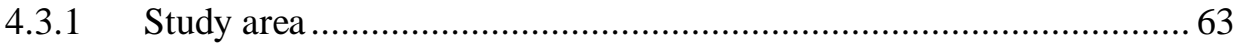

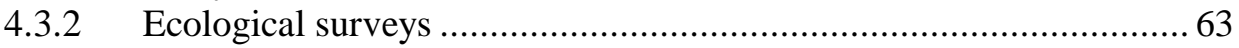

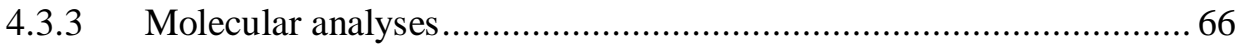

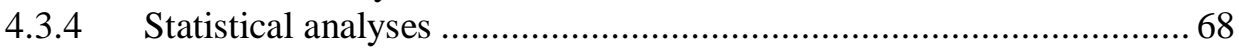

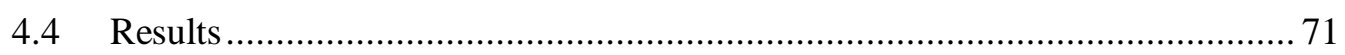

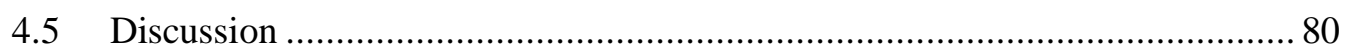

4.5.1 The functional benefits of higher genetic diversity .......................... 81

4.5.2 Mechanisms underlying the relationship between genetic diversity

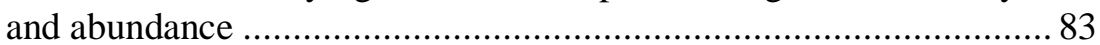

4.5.3 Density-dependent relationships between Anoplolepis gracilipes abundance and native ant community structure and diversity ............ 84

4.5.4 Habitat characteristics may be less important than genetic diversity.. 85

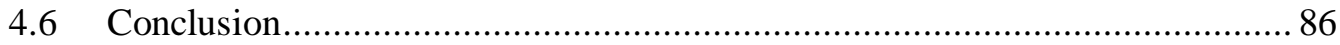


Chapter 5. Population decline but increased distribution of an invasive ant genotype on a Pacific atoll

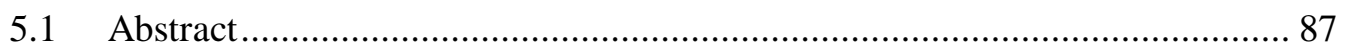

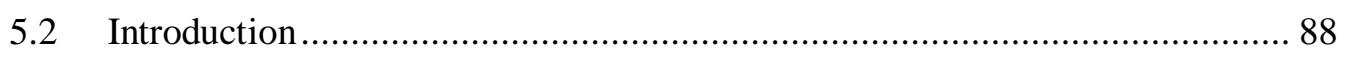

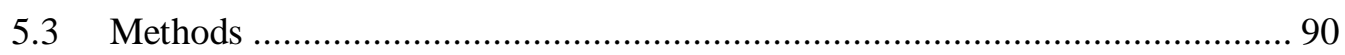

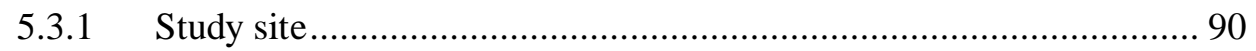

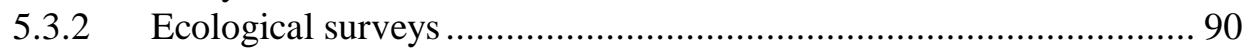

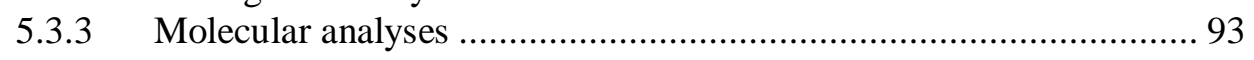

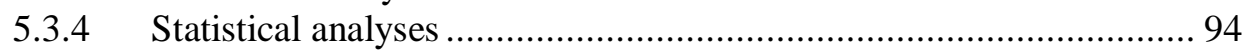

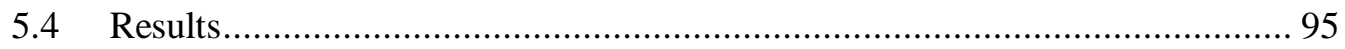

5.4.1 Changes in the distribution of Anoplolepis gracilipes in Tokelau...... 95

5.4.2 Changes in the abundance of Anoplolepis gracilipes on Nukunonu atoll ............................................................................... 98

5.4.3 The association between Anoplolepis gracilipes abundance and effects on the resident ant communities .......................................... 101

5.4.4 Tokelau residents perceptions of the Anoplolepis gracilipes invasion ...

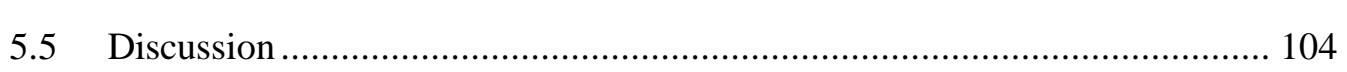

5.5.1 Changes in Anoplolepis gracilipes distribution on Tokelau............. 104

5.5.2 Density-dependent effects of Anoplolepis gracilipes invasion .......... 105

5.5.3 Anoplolepis gracilipes population dynamics................................... 106

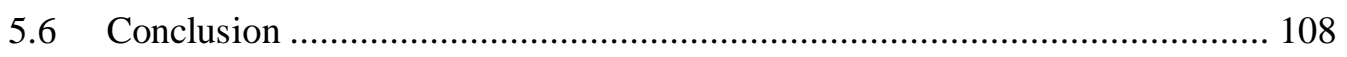

Chapter 6. A preliminary metagenomic survey of Anoplolepis gracilipes endogenous parasite

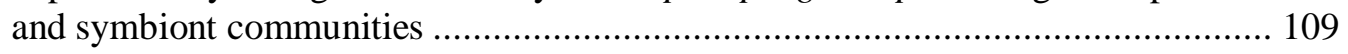

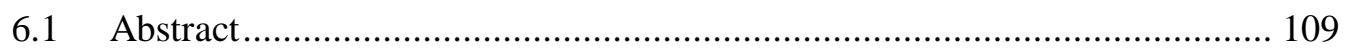

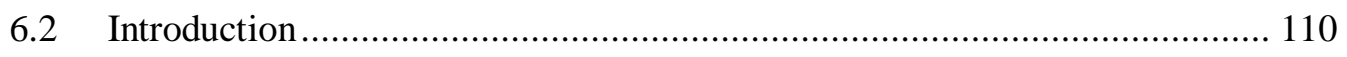

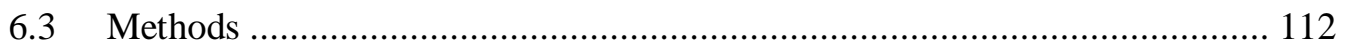

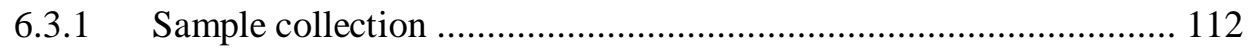

6.3.2 Illumina GAIIx sequencing ................................................ 113

6.3.3 Quality assessment of Illumina GAIIx sequencing ....................... 116

6.3.4 Genomic sequence assembly ................................................ 118

6.3.5 BLAST sequence similarity searching and post-processing ........... 119

6.3.6 Community composition and diversity ....................................... 121

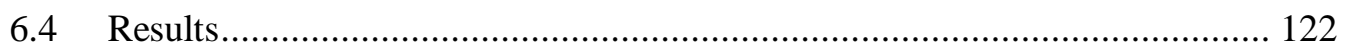

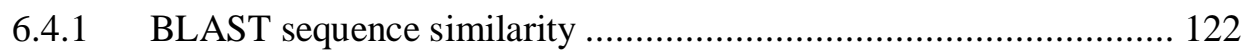

6.4.2 Community composition and diversity ...................................... 123

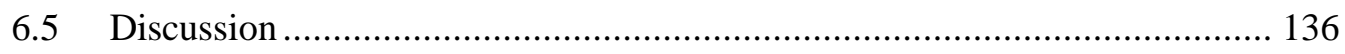

6.5.1 Community composition differences between databases................. 136

6.5.2 The role of Burkholderiales and associated symbionts .................... 137

6.5.3 The role of Rickettsiales........................................................... 139

6.5.4 Enterobacteriales and other common bacterial groups .................. 140

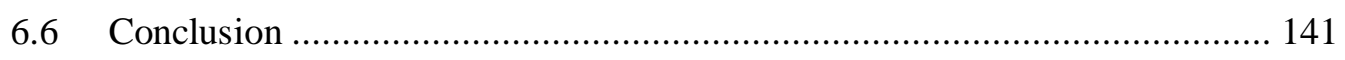




\section{Contents}

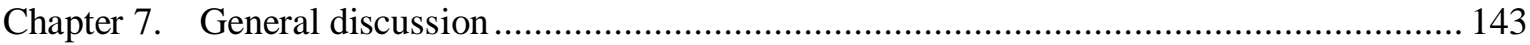

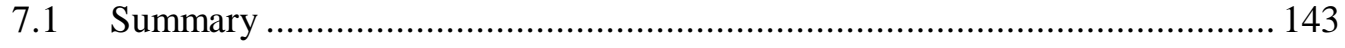

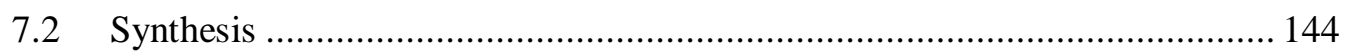

7.3 Constraints and further research ........................................................... 151

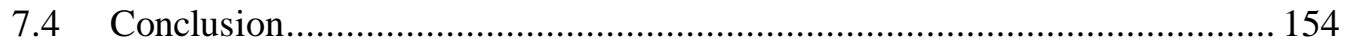

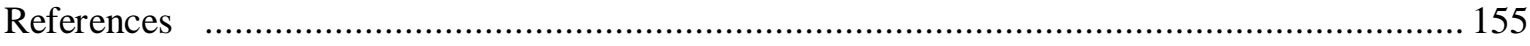

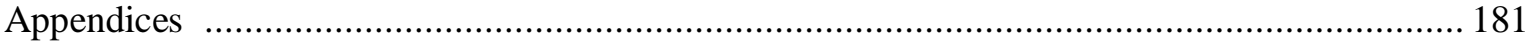

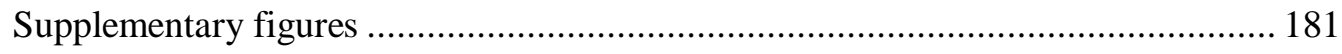

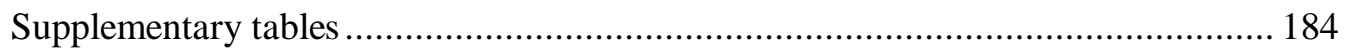

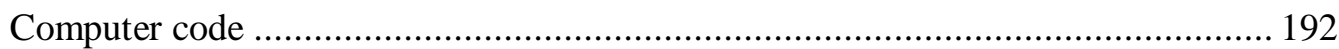

Taxon4blast.pl README .................................................................... 192

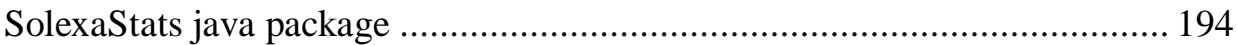




\section{List of figures ${ }^{1}$}

Fig. 2.1: Sampling sites of Anoplolepis gracilipes in Arnhem Land. Grey circles ( $\bigcirc$ ) indicate known locations of $A$. gracilipes. Open circles $(O)$ indicate sites where workers only were sampled in October 2007. Black circles ( ) indicate sites where queens, workers and male brood were sampled in August 2008 and March 2009. One nest was sampled at each site, with the exception of SC, where seven nests were sampled. The black square ( $\square$ ) indicates the site (SH) where workers for dissection and males and were collected in October 2009.

Fig. 2.2: Allele frequencies for the five microsatellite loci (Ano1, Ano3, Ano4, Ano7 and Ano8) used in this study of $A$. gracilipes queens, sperm, males and workers. The vertical dashed lines indicate potentially disjoint allele distributions that typically separated putatively paternal and maternal alleles

Fig. 2.3: Population structure of A. gracilipes queens, workers, sperm and males in Arnhem Land. The hierarchical plot was based on Allele Sharing Distance calculated with Ward's minimum variance algorithm. Queens were found in cluster A, and workers were found in cluster B. Sperm and males were found in both clusters. Details of cluster membership are provided in Fig 2.4....28

Fig. 2.4: Detailed population structure of A. gracilipes queens (Q), workers (W), sperm (S) and males (M) in Arnhem Land. The hierarchical plot was based on Allele Sharing Distance calculated with Ward's minimum variance algorithm, and shows details of cluster membership.

Fig. 2.5: Gaster dissection of A. gracilipes worker, showing ovarioles with no evidence of yolky oocyte development or yellow bodies

Fig. 3.1: Anoplolepis gracilipes distribution and sample sites in Arnhem Land in the Northern Territory, Australia. Pale circles $(O)$ represent the known extent of the invasion at the time of sampling. Black circles (O) are sites sampled in October 2007 for analysis of population structure. Black squares ( $)$ represent the 13 sites sampled in July 2009 for genetic analysis and behavioural assays .42

\footnotetext{
${ }^{1}$ In some instances captions are abbreviated from those in the figures within the chapters
} 
Fig. 3.2: Population genetic structure of A. gracilipes in Arnhem Land. (a) STRUCTURE identified two genetic clusters. (b) Geographical representation of the genetic clusters identified by STRUCTURE analysis. (c) Principal components analysis in PCAGEN revealed no strong clustering of sites. .50

Fig. 3.3: Relationship between Nei's distance and geographical distance among A. gracilipes sites included in behavioural analyses only (a) and all sites (b), with Mantel correlation co-efficients $(r)$, Spearman's correlation co-efficients $\left(r_{s}\right)$ and their respective significance values $(P)$ .51

Fig. 3.4: Boxplot comparing aggression scores of A. gracilipes workers within and between sites. Aggression scores were significantly higher between than within sites (Wilcoxon rank sum test, $Z$ $=-3.80, P<0.001)$.......

Fig. 3.5: Relationships between aggression score and Nei's distance (a) and geographical distance (b) among nests of A. gracilipes Spearman's correlation co-efficients $\left(r_{s}\right)$ and significance values $(P)$ of tests are shown for each plot. 54

Fig. 4.1: Our hypothesised relationships between the genetic diversity (e.g., allelic richness or genotypic richness) and abundance of the invading population, and relative influence on invaded communities under scenarios of: (a) a positive relationship between genetic diversity and abundance; and (b) a negative relationship between genetic diversity and abundance

Fig. 4.2: Anoplolepis gracilipes distribution in Arnhem Land in the Northern Territory, Australia at the time of sampling in July 2009 (grey circles), and sampling sites (black squares with site codes)

Fig. 4.3: The relationship between A. gracilipes abundance and genetic diversity measures: (a) ${ }^{0} D$ (allelic richness); (b) ${ }^{1} D$ (exp[Shannon's H']); (c) ${ }^{2} D$ (1/Simpson's index); (d) genotypic diversity.

Fig. 4.4: Three-dimensional MDS plot of A. gracilipes invaded and uninvaded plots based on Bray-Curtis similarity of $\log (\mathrm{X}+1)$ transformed abundance data. Anoplolepis gracilipes were excluded from the analysis

Fig. 4.5: Relationships between A. gracilipes abundance and native ant: (a) ${ }^{0} D$ (species richness); (b) ${ }^{1} D$ (exp[Shannon's H']); (c) ${ }^{2} D(1 /$ Simpson's index... .79 
Fig. 5.1: Map of the location of: (a) the Tokelau atolls in the Pacific Ocean; and (b) Nukunonu atoll, showing the detections of Anoplolepis gracilipes in 2011, and the invasion status of each islet with reference to the earlier survey in 2004.

Fig. 5.2: Parsimony network describing the relationships between A. gracilipes haplotypes detected in Tokelau in 2004 and 2011. The number of samples included for each haplotype is given in brackets. Nucleotide substitution differences between haplotypes and their position in the sequence fragment are indicated...

Fig. 5.3: Interaction plot comparing differences in mean pitfall trap abundance (per trap) of $A$. gracilipes haplotype A (open circles) and D (closed circles) in 2004 and 2011 on Nukunonu atoll, Tokelau. .98

Fig. 5.4: The relationships between A. gracilipes abundance (total number of ants per invaded plot) and native ant species richness in: (a) 2004; and (b) 2011.

Fig. 5.5: Two-dimensional MDS plots of Nukunonu, Tokelau ant communities comparing differences between: (a) sites in 2004 and 2011; (b) Anoplolepis gracilipes invaded and uninvaded sites in 2011; and (c) Anoplolepis gracilipes haplotypes in invaded sites in 2011...100

Fig. 6.1: Anoplolepis gracilipes sampling sites in Okinawa, Samoa, Christmas Island, and Arnhem Land in the Northern Territory, Australia. In Arnhem Land and on Christmas Island black circles (-) indicate sites that were sampled with high A. gracilipes abundance, and white circles $(O)$ indicate sites that were sampled with low ant abundance. .114

Fig. 6.2: Two-dimensional MDS plots of differences in A. gracilipes endogenous bacterial community composition based on BLAST search results.

Fig. 6.3: Summary of BLAST matches to bacterial orders in the 16SMicrobial database for five $A$. gracilipes samples....

Fig. 6.4: Summary of BLAST matches to bacterial orders in the wgs database for five $A$. gracilipes samples.

Fig. 6.5: Summary of BLAST matches to bacterial orders in the other_genomic database for five A. gracilipes samples

Fig. 6.6: Summary of BLAST matches to bacterial orders in the nt database for five A. gracilipes samples. 
Supplementary Fig. 4.1: Allele discovery curves for the most polymorphic locus (Ano8) for all nine Anoplolepis gracilipes invaded sites (18 plots) in the study, based on the complete dataset of 539 ants.

Supplementary Fig. 4.2: Species accumulation curves based on pitfall trap catches for $A$. gracilipes: (a) invaded; and (b) uninvaded sites in Arnhem Land, Australia...... 182

Supplementary Fig. 4.3: Principal component analysis of A. gracilipes invaded and uninvaded plots based on Euclidean distance of standardised values for the availability of nest sites, canopy cover, presence of Acacia spp. and leaf litter depth 183 


\section{List of tables ${ }^{1}$}

Table 2.1: Genetic diversity estimates for Anoplolepis gracilipes queens (Q), workers (W), sperm (S) and males (M) in Arnhem Land. Values given for heterozygote excess (E) or deficiency (D) represent $P$ values. NA indicates values could not be determined as the locus showed no variation for the group. .22

Table 2.2: Multi-locus genotypic patterns among A. gracilipes queens, sperm, males and workers. Alleles have been encoded to letters for ease of interpretation. A, $a=A n o 1, B, b=$ Ano3, $C, c=$ Ano4, $D, d=$ Ano $7, E, e=A n o 8,-1=$ failed to amplify. .25

Table 2.3: Examples of genotypes of males, queens, workers and sperm for three A. gracilipes nests sampled in Arnhem Land, indicative of the genotypic patterns found among nests....

Table 3.1: Relatedness $r$ values (lower left diagonal, plain font) and mean aggression scores (upper right diagonal, italic font) of within and between site behavioural trials of A. gracilipes workers.

Table 3.2: Abundance and relatedness estimates for A. gracilipes from the 13 sites included in behavioural trials. Abundance, mean $\pm \mathrm{SE}$ and co-efficient of variation $(\mathrm{CV})$, measured using card counts. Values of zero reflect non-detection due to low density, rather than absence; N, number of individuals genotyped; Wang's $r$, mean within nest relatedness \pm SD (range)

Table 3.3: Microsatellite marker diversity estimates for A. gracilipes workers from 20 sites. N, number of individuals genotyped; Alleles, number of alleles detected per locus; $\mathrm{AR}_{10}$, Allelic richness adjusted to the smallest sample size $(\mathrm{n}=10) ; H_{\mathrm{E}}$, expected heterozygosity; $H_{\mathrm{O}}$, observed heterozygosity; $P_{\mathrm{HW}}$, significance values for tests of Hardy Weinberg proportions (heterozygote excess

Table 4.1: Abundance and genetic diversity parameters for Anoplolepis gracilipes in 18 invaded plots in Arnhem Land. Abundance = total number of ants caught in pitfall traps in the plot, $\mathrm{N}=$ number of workers genotyped, Allelic richness = rarefied allelic richness for each locus based on the smallest sample size $(21),{ }^{0} D=$ rarefied allelic richness for the three informative loci, ${ }^{l} D=$ $\exp \left[\right.$ Shannon's H']), ${ }^{2} D=(1 /$ Simpson's index $), G=$ multi-locus genotypes, $R_{U}=$ unbiased genotypic diversity. .73

\footnotetext{
${ }^{1}$ In some instances captions are abbreviated from those in the tables within the chapters
} 


\section{List of tables}

Table 4.2: Differences in the abundances (log-transformed) of the ant species and functional groups that contributed to $\sim 90 \%$ of the dissimilarity between A. gracilipes invaded sites compared with uninvaded sites in Arnhem Land, Australia..................................76

Table 5.1: Comparison of the genetic haplotypes and total pitfall trap abundance per site of Anoplolepis gracilipes on invaded islets on Nukunonu Atoll, Tokelau in 2004 (Abbott et al. 2007) and 2011.

Table 5.2: Comparison of the ant species caught in pitfall traps on Nukunonu Atoll, Tokelau in 2004 (Abbott et al. 2007) and 2011 102

Table 6.1: Sample name, locations sampled, number of sites per location, and total number of Anoplolepis gracilipes ants from which DNA was extracted for next generation Illumina GAIIx sequencing.

Table 6.2: Results from Illumina GAIIx sequencing and quality filtering of A. gracilipes samples

Table 6.3: Velvet assembly results for Illumina GAIIx sequencing data generated from five pooled samples of $A$. gracilipes populations.

Table 6.4 (a): BLAST results for bacterial genera sequences in the GenBank 16SMicrobial and wgs databases for five A. gracilipes samples including number of matches, sum of the lengths of matches and number of genera.

Table 6.4 (b): BLAST results for bacterial genera sequences in the GenBank other_genomic and nt databases for five A. gracilipes samples including number of matches, sum of the lengths of matches, and number of genera.

Table 6.5: The 14 most common genera matched by BLAST searches in the GenBank 16SMicrobial database for the five A. gracilipes samples, the total number of matches, and total length of matches for each sample, ordered by their prevalence in the Arnhem Land 4 sample.

Table 6.6: The 14 most common genera matched by BLAST searches in the GenBank wgs database for five A. gracilipes samples, the total number of matches, and total length of matches for each sample.

Table 6.7: The 14 most common genera matched by BLAST searches in the GenBank other_genomic database for five A. gracilipes samples, the total number of matches, and total length of matches for each sample. 
Table 6.8: The 14 most common genera matched by BLAST searches in the GenBank nt database for five A. gracilipes samples, the total number of matches, and total length of matches for each sample.

Supplementary Table 2.1: Allele frequencies and sample sizes for Chapter 2

Supplementary Table 3.1 (a): Allele frequencies and sample sizes for Chapter 3. Plots A1 D2.

Supplementary Table 3.1 (b): Allele frequencies and sample sizes for Chapter 3. Plots D3 K4 186

Supplementary Table 3.1 (c): Allele frequencies and sample sizes for Chapter 3. Plots K6 $\mathrm{P} 2$.

Supplementary Table 4.1 (a): Allele frequencies and sample sizes for Chapter 4. Plots A1B3.

Supplementary Table 4.1 (b): Allele frequencies and sample sizes for Chapter 4. Plots D2 G1 189

Supplementary Table 4.1 (c): Allele frequencies and sample sizes for Chapter 4. Plots G2 K6. 190

Supplementary Table 4.1 (d): Allele frequencies and sample sizes for Chapter 4. Plots M2 Y3. 


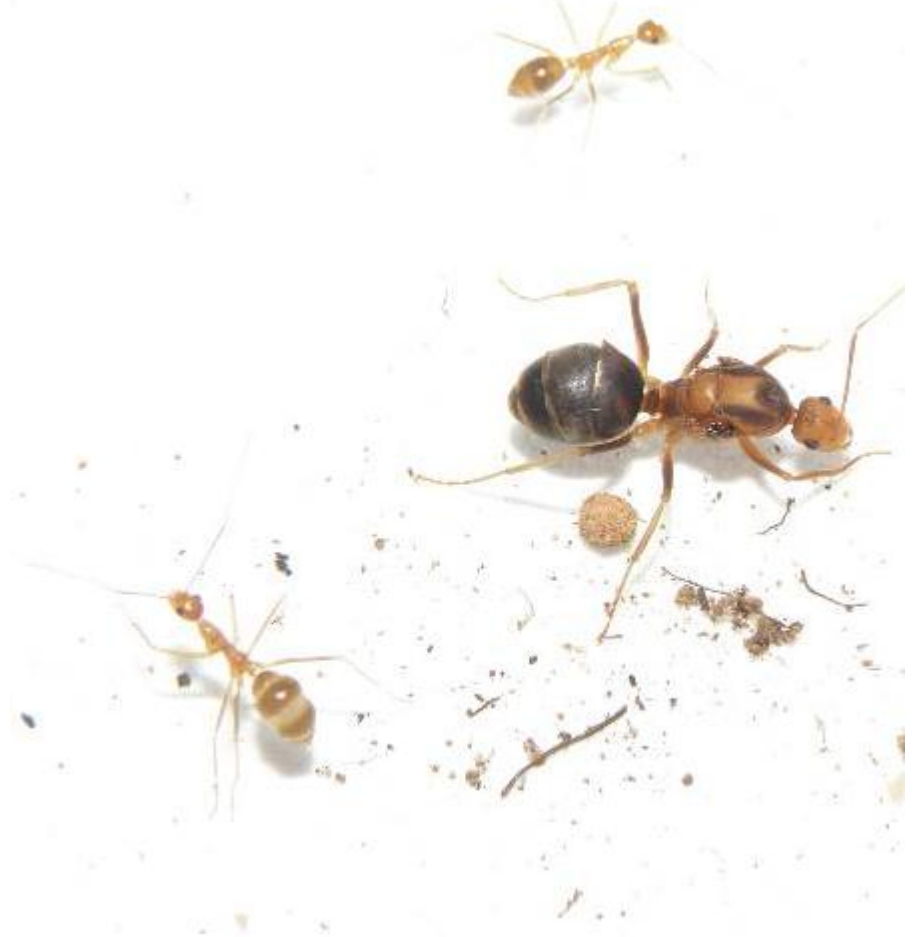




\section{Statement of authorship}

Chapters 2 to 6 of this thesis were written as independent manuscripts intended for publication. As these chapters included contributions of collaborators, they were written in first person plural tense. In all cases I wrote the manuscripts. All three supervisors provided advice throughout and contributed to editing of the manuscripts, and are therefore all co-authors, with the exception of Chapter 5. The contributions of other coauthors to each chapter are outlined below, indicated by author initials ${ }^{3}$.

Chapter 2 author contributions: MG designed the research and formulated the research questions. MG and $\mathrm{BH}$ collected the samples, and $\mathrm{BH}$ conducted initial dissections of workers. MG conducted the genetic analysis, remainder of dissections, analysed the data, and interpreted the results.

Chapter 3 was accepted for publication on June $2^{\text {nd }} 2011$ and is published in Diversity and Distributions 18:323-333. Author contributions: MG, PL and BH designed the research and formulated the research questions. MG and $\mathrm{BH}$ collected the samples. MG conducted the behavioural assays, genetic analysis and data analyses, and interpreted the results.

Chapter 4 was accepted for publication in Ecology and Evolution with minor revisions on May $16^{\text {th }}$ 2012. Author contributions: MG and PL designed the research. MG formulated the research questions. $\mathrm{MG}$ and $\mathrm{BH}$ undertook the field survey. MG conducted genetic analyses, ant identifications, analysed the data, and interpreted the results.

\footnotetext{
${ }^{3}$ Author initials and names: AB Allan Burne; AF Anna Friedlander; BH Ben Hoffmann; CJ Cameron Jack;
} KA Kirsti Abbott; MG Monica Gruber; PL Phil Lester; PR Peter Ritchie; RP Ray Pierce 
Chapter 5 was accepted for publication in Biological Invasions with minor revisions on May $17^{\text {th }}$ 2012. Author contributions: MG formulated the research questions and designed the research based on the earlier work of KA and PL. MG, AB and RP undertook the field study. KA and PL provided data from the earlier work. AB and MG conducted genetic analysis and ant identifications. MG analysed the data and interpreted the results.

Chapter 6 author contributions: MG designed the research and formulated the research questions. AF wrote Perl scripts as specified by MG. CJ wrote the function SolexaStats as specified by MG. MG, PL and BH collected samples. MG conducted lab work and analysis, and interpreted the results. 


\section{Chapter 1. General introduction}

Populations colonising a new area, whether they are invasive species, natural arrivals, or populations translocated for conservation, face similar challenges. Founding population sizes are often small, and the new environment is likely to differ in some way from the environment in which the organism evolved, which together may present major obstacles. Extrinsic factors such as climate, habitat and ecological interactions, and intrinsic factors such as genetic variation and life history traits, may influence not only the successful establishment and persistence of the population, but also on-going population dynamics. It has long been speculated that genetic variation and evolutionary changes may contribute significantly to the success of invasive species (Baker 1965; Lewontin 1965; Mayr 1965). My thesis focuses on how genetic factors may affect the variation in abundance (i.e., differential invasion success) of the yellow crazy ant, Anoplolepis gracilipes (Smith 1857).

\subsection{Abundance and invasion success}

Ideally, invasions are best studied in the context of how populations in the introduced range differ from those in the native range (Elton 1958; Hierro et al. 2005; Suarez and Tsutsui 2008; Sanders and Suarez 2011). This enables inferences to be made regarding how ecological differences and evolutionary changes between the ranges may have contributed to invasion success. Invasive ants exhibit many differences in ecology, population structure and behaviour between their native and introduced ranges, which have made it possible to identify factors that have contributed to their success in novel environments (Ross et al. 1996; Porter et al. 1997; Suarez et al. 1999; Tsutsui and Case 2001; Tsutsui et al. 2003a; Foucaud et al. 2006; LeBrun et al. 2007). However, comparisons between the ranges are impossible when the native range is not known. This is unfortunately the case for A. gracilipes. Although its congeners are African, the ant may have Asian origins (Wetterer 2005), which is supported by some evidence (Drescher 2011; Sebastien et al. 2012). For example, the closest relatives of some endosymbionts of A. gracilipes or the hosts of those endosymbionts appear to be from Asia (Sebastien et al. 2012), and lower diversity at mitochondrial markers in Indo-Pacific populations suggests 


\section{Chapter 1}

that these may have arisen from populations in Asia (Drescher 2011). However, the entire range is not known and has not been sampled. In particular, no information exists regarding the population genetic structure or behaviour of the ant in India (Drescher 2011; H. Feldhaar, personal communication).

So without reference to the native range how can we define and measure invasion success? Without the ability to compare with the native range, it is not possible to determine changes that may have facilitated invasion. In addition, merely the fact that a species is apparently successful in the introduced range is not, on its own, a sufficient criterion to judge any specific characteristics as factors contributing to invasion success. Although invasion success is difficult to definitively quantify, and impossible to predict over the long term, the ability to reach sufficient abundance to persist, spread, and alter or dominate the recipient biological community is a key component to the success of many invasive species (Ehrlich 1986; Crawley 1987; Mack et al. 2000). This is clearly true of invasive ants. Overwhelming evidence suggests that the ecological success of invasive ants is owing to their ability to attain significantly high numbers, and thus dominate the novel ecological community (Macom and Porter 1996; Ross et al. 1996; Holway 1999; Human and Gordon 1999; Morrison 2000; Hill et al. 2003; O'Dowd et al. 2003; Abbott 2005; Le Breton et al. 2005; Rowles and O'Dowd 2007; Bos et al. 2008; Krushelnycky and Gillespie 2008; Lester et al. 2009; Sagata and Lester 2009; Krushelnycky et al. 2010). Mechanistically, higher abundance of invasive ants confers stronger direct competitive ability relative to native ants (e.g., Human and Gordon 1999; Morrison 2000), typically through reduced intra-specific aggression (e.g., Holway et al. 1998; Suarez et al. 1999; Steiner et al. 2007). Thus, for the purpose of this thesis I define invasion success at a given point in time as the ability of the invading population to reach sufficient abundance to cause negative ecological effects in the community to which it is introduced.

At very high abundances the effects of invasive ants can be severe. Disassembly of resident ant communities may occur (Porter and Savignano 1990; Sanders et al. 2003; Lester et al. 2009), and in extreme cases invasions can negatively affect ecosystem functioning (O'Dowd et al. 2003; Davis et al. 2008). However, when in low abundance, invasive ants may not be able to compete successfully with native ants or dominate 
resources (Oliver et al. 2008; Sagata and Lester 2009). Hence, any factors that positively or negatively affect the abundance of the invading ant population, and thus its competitiveness, will affect invasion success. The comparison of more and less abundant populations in the invaded range may provide insight into why some populations persist while others fail (or decline).

A number of factors contribute to the ability of invasive ants to reach high abundance (reviewed by Krushelnycky et al. 2010). One of the key drivers of the ecological success of ants is their advanced social organization (Wilson 1987). This social organisation can be viewed as a continuum from multicoloniality, where colonies are made up of a single nest, whose members behave aggressively to all outsiders, to unicoloniality, where ants display little or no aggression among many nests over the entire population, over a wide geographical area (Bourke and Franks 1995; Pedersen et al. 2006; Suarez et al. 2008). Although unicoloniality is found among ants that are not invasive, many invasive ants are unicolonial. Indeed, unicoloniality is considered to be one of the most important traits promoting the abundance and ecological success of many invasive ants (Helanterä et al. 2009; Krushelnycky et al. 2010).

\subsection{Genetic factors and the success of invasive ants}

Introduced populations face significant genetic challenges, and it has long been speculated that genetic variation and evolutionary changes may contribute to the success of invasive species (Baker 1965; Lewontin 1965; Mayr 1965). The success of invasive species has been called a genetic paradox (Allendorf and Lundquist 2003). This is because initial founding populations may experience extreme genetic bottlenecks, or founder effects, owing to a limited number of colonisers carrying only a small proportion of the genetic variation of the source population (Mayr 1942). Small founding populations are susceptible to the effects of a random change in allele frequencies over several generations (i.e., genetic drift), which most often leads to the rapid loss of rare, but potentially important genetic variation (Fisher 1930; Wright 1931; Nei et al. 1975). Founder effects and strong or repeated bottlenecks have a number of ecological and evolutionary consequences (Frankel and Soulé 1981; Sakai et al. 2001). In the short term, the probability of inbreeding, which potentially leads to reduced fitness owing to 


\section{Chapter 1}

inbreeding depression (Keller and Waller 2002), increases with a loss of allelic diversity as individuals become more closely related (Lande 1988; Frankham 2005). Susceptibility to disease may also increase through a loss of resistance conferred by variation at immune-related loci (Piertney and Oliver 2005; Luquet et al. 2012), and genetic diversity is known to enhance disease resistance among invertebrates (O'Brien and Evermann 1988; Altermatt and Ebert 2008; Lefêvre et al. 2011). The rate of evolutionary change in response to natural selection is proportional to the amount of additive genetic variance in the population (Fisher 1930). As bottlenecks are expected to decrease additive genetic variance (Chakraborty and Nei 1982; Lynch and Hill 1986), the population's adaptive potential is hindered (Willi et al. 2006), and extinction risk is increased (Frankham 2005). Despite these apparent disadvantages, invasive populations sometimes even undergo adaptive evolution (reviewed by Lee 2002; Dlugosch and Parker 2008), or potentially experience greater success owing directly to genetic bottlenecks (Suarez et al. 1999; Tsutsui et al. 2000).

Genetic changes in populations in the introduced range of invasive ants have been hypothesised to promote or magnify unicoloniality, whereby ants that are genetically similar perceive each other as related, although they are not. This may occur through a general loss of genetic diversity (Tsutsui et al. 2000), a specific loss of variation at recognition loci (Giraud et al. 2002; Ross et al. 2003), or the action of green beard (Hamilton 1964; Dawkins 1976) alleles (Keller and Ross 1998). For example, it has been suggested that in the invasive Argentine ants (Linepithema humile), a general loss of genetic diversity owing to population bottlenecks has promoted large-scale unicoloniality, and thus the ecological success of these ants (Suarez et al. 1999; Tsutsui et al. 2000; Tsutsui and Case 2001; Tsutsui et al. 2003b; Brandt et al. 2009). Alternatively it has been suggested that bottlenecks have resulted in genetic cleansing at specific recognition alleles, which may have led to unicoloniality (Giraud et al. 2002). Others disagree that a loss of genetic diversity has stimulated unicoloniality in this species (Vogel et al. 2010), based on the fact that unicoloniality also occurs in the native range (Pedersen et al. 2006). However, there are clear differences in the scale of colony size, genetic diversity, behaviour and ecology of this species throughout the introduced range (Suarez et al. 1999; Giraud et al. 2002; Corin et al. 2007; Brandt et al. 2009; Suhr et al. 2009; van Wilgenburg et al. 2010). Such changes or plasticity in social structure are not limited to 
Argentine ants (Ross et al. 1996; Cremer et al. 2008; Suarez and Tsutsui 2008). In a group such as ants, where genetic factors may govern sociality and aggression (Hamilton 1964), it is intuitive that genetic and evolutionary factors should play an important role in the invasion success of these species (Ross et al. 1996; Cremer et al. 2008; Suarez and Tsutsui 2008).

A loss of genetic diversity may provide a number of other positive benefits in the invaded range. Typically, rare alleles are most likely to be lost through genetic drift following the founder event (Nei et al. 1975), which may be of benefit to the population if deleterious alleles are purged (Schmid-Hempel et al. 2007). Bottlenecks may also result in an increase in adaptive potential by disrupting the evolutionary inertia of co-adapted gene networks, which has been suggested as a mechanism for a number of models of speciation and adaptation, including Wright's shifting balance theory (Wright 1931), and founder flush speciation models (Mayr 1963; Carson 1968; Templeton 1980). These models predict that bottlenecks and genetic drift enable populations to reach new adaptive peaks, but they are controversial (Cheverud and Routman 1996; van Heerwaarden et al. 2008). In addition, depending on whether serial bottlenecks occur, the effects of additive variance and epistatic variance may differ (Goodnight 1987), as may the conversion from non-additive to additive variance (Goodnight 1988).

Genetic diversity may also be unimportant if asexual reproduction enhances invasion success. In asexually reproducing species, highly adapted clonal lineages can be shielded from potentially harmful sexual recombination events by population bottlenecks (Liu et al. 2006; Mergeay et al. 2006). Clonal reproduction is found in some populations of the invasive little fire ant (Wasmannia auropunctata), while other populations reproduce sexually (Fournier et al. 2005; Foucaud et al. 2006; Foucaud et al. 2007). As clonal populations of this ant are more prevalent in the introduced range, this reproductive mode may contribute to invasion success (Foucaud et al. 2010). It has been suggested that the ecological success of other invasive ants may also be facilitated by clonal reproduction (Kellner and Heinze 2010; Pearcy et al. 2011), although these studies have not investigated the native and introduced ranges, often an important comparison to make when assessing invasion success (Elton 1958; Hierro et al. 2005; Sanders and Suarez 2011). 


\section{Chapter 1}

Among the mechanisms invoked to explain why there is frequently no genetic paradox is propagule pressure, which is one of the most commonly observed factors associated with invasion success generally (Von Holle and Simberloff 2005; Colautti et al. 2006; Dlugosch and Parker 2008; Simberloff 2009; Wilson et al. 2009b). Propagule pressure owing to a large initial founding population size or multiple introduction events (Simberloff 2009; Wilson et al. 2009b) may result in higher genetic diversity in the introduced range than the native range, and thus promote invasion success (Dlugosch and Parker 2008). Multiple introduction events can increase the overall population size, thereby reducing the potential negative effects of genetic drift (Allendorf and Lundquist 2003; Roman and Darling 2007; Facon et al. 2008). Moreover, in some cases, multiple introductions can increase the genetic diversity of the introduced population relative to native populations through inter- or intra-specific hybridisation (Ellstrand and Schierenbeck 2000; Kolbe et al. 2004; Petit 2004; Facon et al. 2008). Although propagule size may affect the establishment of invasive ants (Holway et al. 2002; Sagata and Lester 2009; Yang et al. 2012), populations may also require only a few founders (Hee et al. 2000; Corin et al. 2007; Ross and Shoemaker 2008; Foucaud et al. 2010). To date, no studies have suggested that inter- or intra-specific hybridisation contributes to the success of invasive ants. By contrast, hybridisation may negatively affect invasion success. A number of ant species are known to hybridise, often with negative fitness consequences for the hybrid population (Feldhaar et al. 2008). Some ant species appear to avoid these fitness costs via dependent lineage genetic caste determination (DL GCD) (Feldhaar et al. 2008). The DL GCD mode of reproduction (Anderson et al. 2006) requires obligate hybridisation of two lineages (typically species) to produce workers, while pure lineages become queens (Helms Cahan et al. 2002; Volny and Gordon 2002; Helms Cahan and Vinson 2003). Queens must therefore mate with males from each lineage to produce both workers and reproductives. If the ratio of the two lineages present in the population is skewed, colony failure rates increase (Anderson et al. 2006; Schwander et al. 2006). Ants with DL GCD may thus have lower chances of invasion success if one lineage is underrepresented. 
In some cases, specific biotypes or genotypes may be more invasive than others, while genetic diversity per se does not influence invasion success (Perring 2001; Saltonstall 2002; Petit 2004; Abbott et al. 2007). Non-native, genotypically distinct populations of the reed grass Phragmites australis in North America have faster rates of spread, and are able to outcompete native populations (Saltonstall 2002). Similarly, the whitefly (Bemisia tabaci species complex) has a number of genetic biotypes, of which only two have attained pest status (Perring 2001). Although invasive and non-invasive Bemisia biotypes carry different communities of endosymbionts, including the sex-manipulating Wolbachia, Rickettsia and Arsenophonus (Gueguen et al. 2010; Thierry et al. 2011), it is not known how these might affect fitness in these Bemisia study populations (Gueguen et al. 2010; Thierry et al. 2011). In other invasive whitefly populations Rickettsia has positive effects on both its own and its host fitness through host sex-ratio manipulation towards females, which increases the rate of host reproduction and spread (Himler et al. 2011). Genotype-specific invasion success may also be experienced by the invasive yellow crazy ant $A$. gracilipes, in which one distinct haplotype has been implicated in the high abundance of specific populations of the ant in Tokelau (Abbott et al. 2007). In addition, A. gracilipes harbours a number of endosymbionts, including the sex-ratio manipulators Arsenophonus and Wolbachia (Sebastien et al. 2012), although their function in ants is unknown.

Finally, the molecular markers typically used to estimate genetic diversity might not always directly relate to establishment success and evolutionary potential, as they might not accurately represent the total diversity in the genome, particularly regarding quantitative traits (Väli et al. 2008). The variation in quantitative traits may not be affected by bottlenecks because the distributions of quantitative variation are less sensitive to the loss of rare alleles than molecular markers (Lande 1980; Barton and Charlesworth 1984). 


\section{Chapter 1}

\subsection{Ecological factors also contribute to the invasion success of ants}

Ecological differences in the novel environment, or demographic and environmental stochasticity may be stronger influencers of population persistence than genetic factors (Lande 1988). While my thesis research does not focus on the many ecological mechanisms that might or might not affect the invasion success of A. gracilipes, the role of endogenous diversity (symbionts, parasites and pathogens) is of interest, as these endogenous organisms may affect variation in abundance, their effects may be affected by genetic diversity of the host (O'Brien and Evermann 1988; Shykoff and SchmidHempel 1991; Altermatt and Ebert 2008; Lefèvre et al. 2011), and they are often inherited through vertical transmission from parent to offspring (Zientz et al. 2005).

The relationship between ants, their parasites and symbionts is complex and not well understood compared to other ecological interactions. Although endogenous bacteria may be harmful to their insect hosts (Wenseleers et al. 2002; Schlüns and Crozier 2009), many are also beneficial (Dillon and Dillon 2003; Dale and Moran 2006; Feldhaar and Gross 2009). A number of bacteria play a role in nitrogen metabolism (Kikuchi et al. 2005; Stoll et al. 2007; Russell et al. 2009), and immune defense (de Souza et al. 2009), protecting insects from attack by pathogens, including viruses (Dillon and Charnley 2002; Hedges et al. 2008; Teixeira et al. 2008), and eukaryotic parasites (Currie et al. 1999; Santos et al. 2004; Moran et al. 2005). Parasites may even mediate kin recognition in ants (Martin et al. 2011), and their loss may be associated with invasion success (Cremer et al. 2008; Martin et al. 2011). Invasive ants may lose endosymbionts (e.g., Wolbachia) in the introduced range (Tsutsui et al. 2003a), but the function of common insect endosymbionts such as Wolbachia in ants is unclear and may vary (Zientz et al. 2005). 
Mutualisms, including those with endosymbionts, are often associated with the ecological success of ants, and may contribute to invasion success. Carbohydrate resources obtained through extra-floral nectaries or tending scale insects are known to enhance ant abundance (Davidson 1997 and references therein), and the appropriation of these resources may often involve novel mutualistic interactions in the invaded range (Helms and Vinson 2002; Abbott 2004; Abbott and Green 2007; Wilder et al. 2011). Even ants that are not strictly herbivorous may use nitrogen-fixing endosymbionts to 'upgrade' their nutrition, gaining valuable amino acids that would be otherwise unavailable to them (Feldhaar et al. 2007). Ants may carry a suite of such mutualists, and if their function has a large effect on population dynamics, their loss could also result in population declines (Russell et al. 2009). In the invaded range symbiotic mutualists in particular may be easily lost as their persistence can depend on the host population size (Sachs and Simms 2006). Thus bottlenecks that affect the host may also affect the symbionts. Higher genetic diversity of the host can also enhance parasite resistance (Sherman et al. 1988; Shykoff and Schmid-Hempel 1991; Keller 1995; Tarpy 2003; Ugelvig et al. 2010), so as well as losing mutualists a genetic bottleneck may result in reduced immune function. The changes in the dynamics of these endogenous populations may affect stress tolerance and parasite resistance, and ultimately invasion success (Feldhaar 2011).

Ecological and genetic factors are not necessarily mutually exclusive causes of invasion success, and different contexts may result in different interactions between these mechanisms (i.e., variation in ecological conditions in different parts of the invaded range, variation in propagule size, traits that assist introduction and establishment versus those that assist spread). For example in some contexts a loss of genetic diversity in Spartina sp. is associated with invasion success, while in other cases the opposite is the case (Petit 2004). In Argentine ants, for example, although unicoloniality may have enhanced the success of the ant throughout its invaded range (Suarez et al. 1999; Giraud et al. 2002), perhaps assisted by a loss of natural enemies (Suarez et al. 1999; Tsutsui et al. 2003a), abiotic factors also contribute to its success at the community scale (Menke and Holway 2006), predict its distribution and spread (Krushelnycky et al. 2005; Hartley et al. 2010), and likelihood of persistence (Cooling et al. 2011). 


\section{Chapter 1}

\subsection{Focal study system: Anoplolepis gracilipes in Arnhem Land}

The yellow crazy ant Anoplolepis gracilipes appears among a 'selection of 100 of the world's worst invasive species' (Lowe et al. 2000), and is considered one of the most widespread, abundant and damaging invasive ants (Holway et al. 2002). There is no doubt that $A$. gracilipes can be a serious pest. The ant can occur at very high densities, and its most extreme effects include ecosystem change on Christmas Island, owing to a classic case of 'ecological meltdown', promoted by a mutualism with a secondary introduction of a scale insect (O'Dowd et al. 1999; O'Dowd et al. 2003; Abbott 2006b). However, the abundance of the ant varies spatially and temporally throughout its range (Greenslade 1971; Baker 1976; Abbott 2006b; Abbott et al. 2007; Suwabe et al. 2009), and population declines have also been observed (Haines and Haines 1978b).

Anoplolepis gracilipes was first detected in Northeast Arnhem Land in Australia's Northern Territory around rehabilitated mine sites in 1984 (Majer 1984). By 1989 the ants distribution was estimated to patchily span an area of 2,500 $\mathrm{km}^{2}$ in Arnhem Land (Young et al. 2001), which increased to around $16,000 \mathrm{~km}^{2}$ by 2009 (B. Hoffmann, unpublished data). Anoplolepis gracilipes exhibits varying nest densities and abundance over this wide region. The abundance of $A$. gracilipes in Arnhem Land also appears relatively low compared to populations elsewhere. Carbohydrate resources obtained through novel mutualisms are not an obvious driver of the variation in abundance in Arnhem Land (B. Hoffmann, personal communication), unlike A. gracilipes invasions in Samoa (Savage et al. 2009), and on Christmas Island (O'Dowd et al. 2003). Nor are there obvious associations between some of the more commonly observed factors that contribute to the variation in abundance and success of invasive ants, such as abiotic factors, anthropogenic disturbance, or ecological release. Thus the invasion in Arnhem Land offered the opportunity for me to investigate whether genetic factors might contribute to the variation in abundance of $A$. gracilipes. 
The potential contributions of genetic factors to the invasion success of A. gracilipes have not attracted much study compared to other invasive ants. Studies on this ant have overwhelmingly focussed on the potential ecological causes and effects of its invasion (e.g., Haines and Haines 1978b; Haines et al. 1994; Hill et al. 2003; Gerlach 2004; Green et al. 2004; Lester and Tavite 2004; Abbott 2005; Lach 2005; Ward and Beggs 2007; Bos et al. 2008; Davis et al. 2008; Neville et al. 2008; Pfeiffer et al. 2008; Lester et al. 2009; Matsui et al. 2009; Savage et al. 2009; Brühl and Eltz 2010; Davis et al. 2010; Hoffmann and Saul 2010; Drescher et al. 2011; Lach and Hoffmann 2011; Tanaka et al. 2011). Only recently have studies begun to investigate the genetic aspects of the ants' biology, and these studies have focussed primarily on population structure and behaviour, typically in relation to supercolony structure (Drescher et al. 2007; Drescher et al. 2010; Thomas et al. 2010). One notable exception is the finding that populations of the ant on the Tokelau atolls belonged to either of two common and behaviourally distinct mitochondrial haplotypes (A and D). Haplotype D was associated with more abundant populations and strong negative effects on the resident ant community (Abbott et al. 2007), indicating a potential association between genetic factors and invasion success.

As is often the case with invasive ants, density-dependent invasion success is likely to be generally true also of $A$. gracilipes. On Christmas Island management of the ant is only initiated once abundance has reached a threshold above which ecological effects are observed (Boland et al. 2011), and on Tokelau only more abundant populations have negatively affected resident ant species (Abbott et al. 2007; Lester et al. 2009). Some evidence suggests this is the case in Arnhem Land also (Hoffmann and Saul 2010). Many studies of $A$. gracilipes have focussed on the ant when it is at high abundance (e.g., Hill et al. 2003; Gerlach 2004; Lester and Tavite 2004; Abbott 2005; Bos et al. 2008; Davis et al. 2008; Neville et al. 2008; Lester et al. 2009; Savage et al. 2009; Brühl and Eltz 2010; Drescher et al. 2011). However, the abundance of the ant can vary temporally and spatially, and a few studies have documented this variation in a number of different places (Christmas Island: Abbott 2006b; Tokelau: Abbott et al. 2007; Japan: Suwabe et al. 2009). Reasons suggested for this variation included seasonal differences in ant activity (Abbott 2006b; Suwabe et al. 2009), or haplotype-specific abundance (Abbott et al. 2007). I hypothesised that the association Abbott et al. (2007) found between variation 


\section{Chapter 1}

in abundance and different haplotypes of the ant on Tokelau might also explain the variation in abundance in Arnhem Land.

Populations of $A$. gracilipes also appear to experience unexplained declines or local extinctions. Haines and Haines (1978) noted that A. gracilipes had inexplicably declined on Mahé in the Seychelles. Declines have also been anecdotally reported on Christmas Island (P. Green, personal communication). In both cases the reasons for the declines have not been empirically investigated. In Arnhem Land A. gracilipes has also experienced declines which have no obvious explanation (B. Hoffmann, personal communication). Sudden declines such as these occur occasionally in other invasive species also, and their causes are often unexplained (Simberloff and Gibbons 2004). Populations of $A$. gracilipes are known to harbour a number of endogenous bacteria, including sex-ratio manipulating Arsenophonus, Wolbachia, and herbivory-associated Rhizobiales (Sebastien et al. 2012), although other bacteria and endogenous eukaryotes have not been investigated in the species. The role of these endogenous bacteria in $A$. gracilipes is not known. However, it is possible that variation in microbial communities or bacterial associations with ants influence these population dynamics. Determining which, if any, bacteria are responsible for population declines may take considerable work and time. Here I undertook a study to gain preliminary insights into differences in the microbial communities of A. gracilipes populations. 


\subsection{Thesis aims}

In this thesis I investigate the relationships between the variation in abundance of $A$. gracilipes populations and genetic factors that may affect invasion success. My overall objective is to determine if genetic factors are associated with the observed variation in abundance of A. gracilipes, primarily focussing on populations in Arnhem Land. Each chapter of my thesis addresses a single factor in relation to this objective.

Chapter 2 is concerned with how the reproductive mode might influence the variation in the abundance of $A$. gracilipes, and primarily investigates the possibility of lineageratio asymmetry within dependent lineage genetic caste determination, along with worker reproduction, and discussion regarding asexual reproduction.

In Chapter 3 I investigate the population structure and behaviour of A. gracilipes, to determine if introductions from multiple, different sources contribute to the variation in abundance of the ant in Arnhem Land (i.e., propagule pressure or potentially differential abundance of genetically distinct populations).

Chapter 4 investigates whether there is a positive or negative relationship between genetic diversity and invasion success of A. gracilipes via density-dependent effects on the invaded ant community, and whether selected ecological characteristics are also correlated with variation in abundance.

I revisit the study of Abbott et al. (2007) on Tokelau in Chapter 5 to determine if the A. gracilipes genetic haplotype identified earlier maintains its dominance, and whether the association between high abundance and this dominant haplotype persists. 
Chapter 1

In Chapter 6 I conduct a preliminary investigation of the diversity of endogenous parasites and symbionts of $A$. gracilipes, to identify candidates that may be associated with the variation in abundance of A. gracilipes populations.

Chapter 7 summarises and synthesises the main findings of my thesis, outlines the constraints faced, and opportunities for further research, including additional genetic factors that may affect abundance and invasion success. 


\section{Chapter 2. The conundrum of the Anoplolepis gracilipes reproductive mode: no evidence for dependent lineage genetic caste determination}

\subsection{Abstract}

Asexual reproduction and hybridisation are often found among highly invasive plants and marine invertebrates. Recently, it has been suggested that clonality may enhance the success of invasive ants. By contrast, obligate hybridisation (dependent lineage genetic caste determination or DL GCD in ants) may decrease the chances of population persistence if one lineage is less prevalent than the other (asymmetry in lineage ratio). Genetic characteristics of the invasive yellow crazy ant (Anoplolepis gracilipes) suggest that it has an unconventional mode of reproduction that may involve asexual reproduction by workers or queens, or DL GCD. Anoplolepis gracilipes shows considerable spatial variation in abundance where it has invaded in Arnhem Land in Australia's Northern Territory. Here, we investigated whether A. gracilipes reproduction involved DL GCD, and if so, whether lower abundance could be owing to asymmetry in lineage ratio. The potential for worker reproduction was also assessed. We used microsatellite markers to assess the population structure of $A$. gracilipes workers, males, queens and sperm in queen spermathecae, from field collections in Arnhem Land. We found that a single queen lineage is present in Arnhem Land. The presence of a single lineage of queens discounts the possibility of DL GCD. Population structure separated queens and workers into different lineages, suggesting that these castes are determined genetically in $A$. gracilipes. Evidence for worker reproduction was weak. We conclude that the reproductive mode of $A$. gracilipes does not involve DL GCD, and thus the variation in abundance that we observe in the Arnhem Land population of A. gracilipes is not owing to asymmetry in lineage ratio. The resolution of the reproductive mode of $A$. gracilipes is complicated by a high prevalence of diploid males. The determination of the A. gracilipes reproductive mode remains a fascinating research question, and its resolution may improve our understanding of the contribution of the reproductive system to invasion success. 


\section{Chapter 2}

\subsection{Introduction}

Biological invasions are one of the chief threats to biodiversity, and affect ecosystem function, native organisms and economic well-being (Mack et al. 2000; Pimentel 2005). Although there is little consensus on the mechanisms and predictability of invasiveness, clonal reproduction and hybridisation are commonly observed traits in successful invaders of terrestrial plant and aquatic communities (Ren et al. 2005; Facon et al. 2006; Mergeay et al. 2006). It has become apparent that ant reproductive systems are more diverse than previously thought (Keller 2007; Heinze 2008; Schwander et al. 2010), and recently it has been suggested that the ecological success of some invasive ants may also be facilitated by clonal reproduction (Foucaud et al. 2009; Kellner and Heinze 2010; Pearcy et al. 2011). In clonal genetic caste determination (GCD) systems workers are produced sexually by mated queens, while queens and males are produced clonally. Variations on this system have been found in four species, Cataglyphis cursor (Pearcy et al. 2004), Wasmannia auropunctata (Fournier et al. 2005), Vollenhovia emeryi (Ohkawara et al. 2006; Kobayashi et al. 2008), and Paratrechina longicornis (Pearcy et al. 2011), although the exact mechanisms differ between species. In addition, unmated workers (gamergates) of many species reproduce clonally (reviewed in Heinze 2008).

A number of ant species are known to hybridise, often with negative fitness consequences for the population (Feldhaar et al. 2008). Some ant species avoid the fitness costs of hybridisation via dependent lineage genetic caste determination (DL GCD) (Anderson et al. 2006) The DL GCD system requires obligate hybridisation of two lineages (typically species) to produce workers, while matings within lineages result in queens (Helms Cahan et al. 2002; Volny and Gordon 2002; Helms Cahan and Vinson 2003). Queens must mate with multiple males to produce both workers and other reproductives. Thus the ratio of the two lineages present in the population has consequences for colony fitness. The chance of a queen acquiring sperm from only one lineage increases as the lineage ratio increases in skew, resulting in higher colony failure rates (Anderson et al. 2006; Schwander et al. 2006). Clearly, while clonal reproduction could enhance the chance of successful colonisation and persistence, DL GCD in invasive ants could result in a lower likelihood of invasion success if one lineage is underrepresented. 
The yellow crazy ant (Anoplolepis gracilipes), considered to be one of the most damaging invasive ant species (Holway et al. 2002), has an unresolved reproductive mode. Although the reproductive mode remains elusive, and the ants native range is not known (Wetterer 2005), studies in the invaded range have consistently found that workers are typically heterozygous, queens are typically homozygous, and diploid males are common (Borneo: Drescher et al. 2007; Christmas Island: Thomas et al. 2010). High heterozygote frequencies in workers, and a prevalence of homozygous queens has been interpreted as possibly owing to DL GCD or clonal GCD (Drescher et al. 2007), or asexual reproduction by unmated workers (Heinze 2008). In addition, on Christmas Island at least two mitochondrial lineages of the ant are known to co-exist, although they appear to be reproductively isolated from each other (Thomas et al. 2010). In our study area, Arnhem Land in the Northern Territory of Australia, A. gracilipes worker populations vary spatially in abundance, and we wished to determine if the mode of reproduction contributed to this variation. For example, if $A$. gracilipes reproduction involves a DL GCD system, low abundance could be owing to a persistent skew in lineage ratio.

Here we use molecular markers to test the hypothesis that the reproductive mode of $A$. gracilipes involves DL GCD. If DL GCD was involved we would expect the population genetic structure to reflect queens and males resulting from pure lineages, and workers arising as a result of hybridisation between these pure lineages. It would have been desirable to also investigate the possibility of clonal production of queens and males, however field samples alone are insufficient to conduct the parentage analyses necessary to distinguish clonal versus sexual production of the different castes. We did, however, dissect workers and their ovaries to investigate the possibility of worker reproduction. 


\section{Chapter 2}

\subsection{Methods}

\subsubsection{Study site and sampling}

Anoplolepis gracilipes is patchily distributed throughout $16,000 \mathrm{~km}^{2}$ in Arnhem Land (Fig 2.1). Workers were collected from ten sites throughout the ant's geographical range in October 2007, and queens, workers and brood were collected from an additional ten sites between August 2008 and March 2009 (Fig. 2.1). Samples were collected from a single nest at each site except site SC where we sampled seven nests, spaced 5-100 m apart, on different dates. This sampling regime allowed analysis at a broad (regional) scale, as well as the finer scale at which groups of ants are likely to interact. Nests contained between one and 16 queens (mean $4.1 \pm 0.9 \mathrm{SE}$ ). Ants were stored in 95\% ethanol at $4^{\circ} \mathrm{C}$ until DNA extraction. No adult males were found during the sampling, but four male brood were collected from a nest at site YI3 and one from a nest at site SC. In October 2009 (coinciding with the emergence of A. gracilipes sexuals), 24 males from a single nest (site $\mathrm{SH}$ ) were sampled for genotyping. Workers from 13 nests at this site were also sampled for dissection to investigate the possibility of worker reproduction.

\subsubsection{Genetic analyses}

To determine the genotypes of males contributing to reproduction, we extracted sperm from the spermathecae of queens. Queen gasters were dissected, and the sperm contents extracted from the spermathecae and placed in $95 \%$ ethanol. Contamination by queen tissue from the spermatheca was considered unlikely as the sperm DNA content was likely to be greater than that of the spermathecal wall. Thus, sperm cell DNA would have preferentially amplified under PCR (Krieger and Keller 2000). This assumption was upheld by an earlier test of the method on four queens and their spermathecal contents from Christmas Island (unpublished data) for another project. Three of the four sperm samples had one allele (i.e., were likely to be haploid), which did not match an allele of the queen from which the spermatheca had been dissected. The fourth sperm sample had two alleles, one of which matched an allele of the queen from which it had been dissected. 


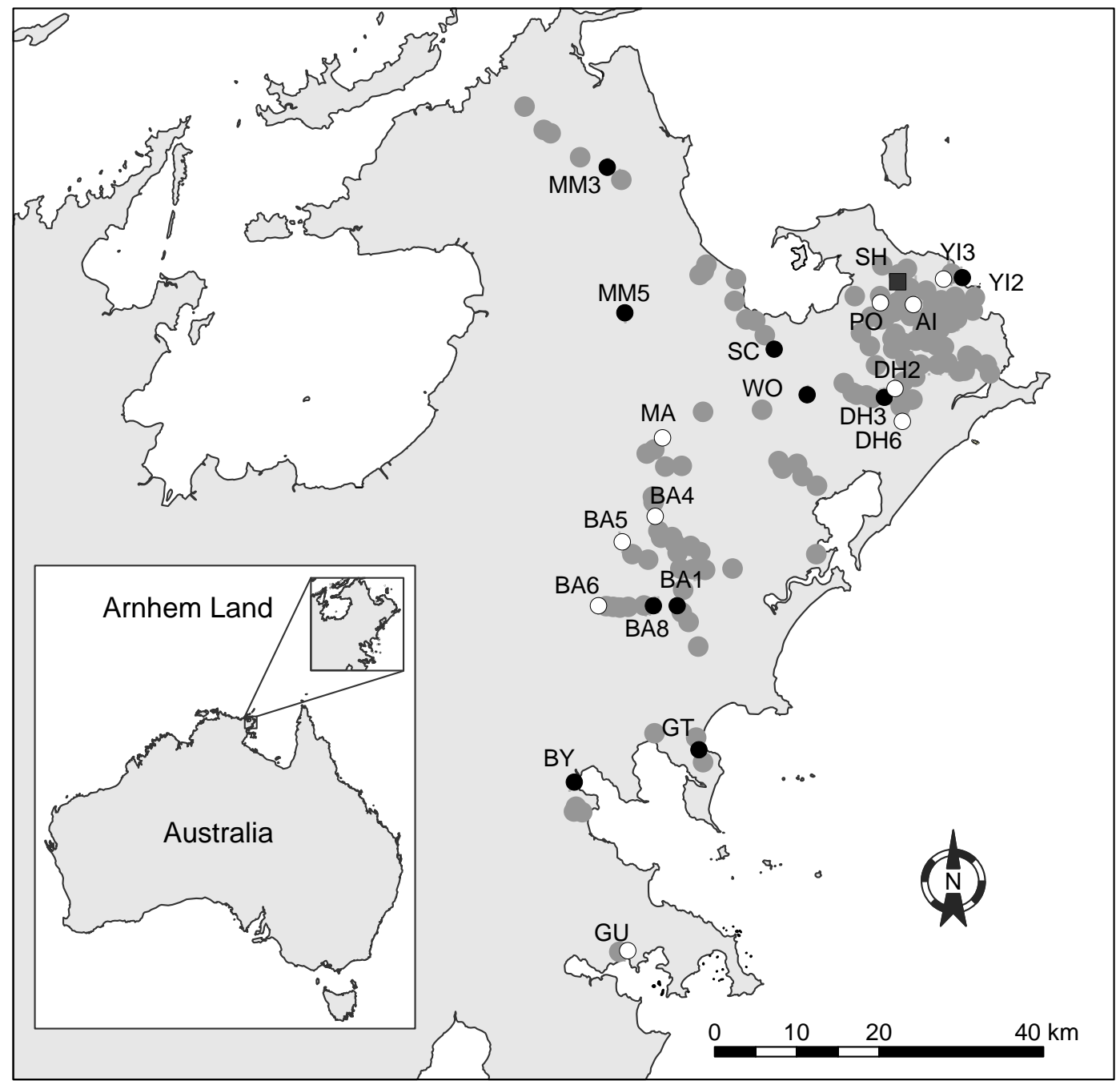

Fig. 2.1: Sampling sites of Anoplolepis gracilipes in Arnhem Land. Grey circles ( $\odot$ ) indicate known locations of $A$. gracilipes. Open circles $(\bigcirc)$ indicate sites where workers only were sampled in October 2007. Black circles ( ) indicate sites where queens, workers and male brood were sampled in August 2008 and March 2009. One nest was sampled at each site, with the exception of SC, where seven nests were sampled. The black square ( $\square$ ) indicates the site (SH) where workers for dissection and males were collected in October 2009. 


\section{Chapter 2}

We extracted DNA using a modified Chelex protocol (Sepp et al. 1994). Spermathecal contents were placed in $1.7 \mathrm{~mL}$ microcentrifuge tubes with $5 \mu \mathrm{lof} 10 \mathrm{mg} / \mathrm{mL}$ Proteinase $\mathrm{K}$ and $60 \mu \mathrm{l}$ of $10 \% \mathrm{w} / \mathrm{v}$ Chelex-100 resin solution and heated for 2 hours at $60^{\circ} \mathrm{C}$. Individual workers, brood, or heads of queens were ground using sterile plastic pestles in microcentrifuge tubes containing $150 \mu \mathrm{l}$ of a $10 \% \mathrm{w} / \mathrm{v}$ Chelex-100 resin solution. Tubes were centrifuged briefly, boiled for 15 minutes, chilled on ice for five minutes and then centrifuged for 15 minutes at $15,000 \times g$ at $4^{\circ} \mathrm{C}$. The supernatant containing DNA was stored at $4^{\circ} \mathrm{C}$.

We used five microsatellite markers, Anol, Ano3, Ano4, Ano7 and Ano8 (Feldhaar et al. 2006), to investigate population structure and identify multiple lineages. Each $15 \mu \mathrm{l}$ PCR consisted of $\sim 20 \mathrm{ng}$ template DNA, 10× PCR Buffer, $0.4 \mathrm{mg} / \mathrm{mL}$ of bovine serum albumin (BSA), 1.5 - $2.5 \mathrm{mM} \mathrm{MgCl}_{2}, 0.2 \mathrm{mM}$ of each dNTP, $0.1 \mathrm{mM}$ of forward primer, $0.4 \mathrm{mM}$ of reverse primer, $0.4 \mathrm{mM}$ of universal fluorescent dye-labelled M13(-21) primer (Schuelke 2000) and 0.1 U of Taq DNA Polymerase (New England Biolabs). Thermal cycling was performed on an Eppendorf 2700 thermal cycler using conditions specified by Feldhaar et al. (2006), with modification for M13(-21) primers (Schuelke 2000). Amplified products were analysed on a 3730 Genetic Analyzer and genotypes scored using Genemapper v 3.7 (Applied Biosystems). Successful genotyping of 16 nests included three to 12 workers (mean $\pm \mathrm{SE}=6.1 \pm 0.7, \mathrm{n}=96$ ), one to 10 spermathecal contents (mean $\pm \mathrm{SE}=3.6 \pm 0.6, \mathrm{n}=68$ ), and one to 11 queens (mean $\pm \mathrm{SE}=4.1 \pm 0.9, \mathrm{n}$ $=73$ ). Fifteen to 20 workers (mean $\pm \mathrm{SE}=19.4 \pm 0.4, \mathrm{n}=214$ ) were genotyped from 11 nests without queens. Four male brood from one nest and one from a second nest were also genotyped, along with the 24 adult males from a single nest. We used Micro-Checker v 2.2.3 (van Oosterhout et al. 2004) to validate genotyping quality. Scoring errors, presence of null alleles and large allele dropout were not apparent.

Sperm with homozygous multi-locus genotypes were considered to be haploid (hemizygous). Sperm with heterozygous genotypes at one or more loci were either diploid, and / or the queen was multiply mated, or the sperm sample was contaminated by queen tissue. We visually inspected the sperm genotypes to detect features that could indicate contamination. It was decided that sperm samples with relatively low volume that had a multi-locus genotype matching the genotype of the queen from which they 
were dissected could be unreliable results and should be excluded from analysis. We excluded one sperm sample on this basis. In addition, sperm with genotype scoring peaks that were substantially lower for maternal alleles were also considered unreliable (i.e., we would expect maternal alleles to be non-preferentially amplified if the queen contribution of DNA from the spermatheca was much lower than the sperm contribution). No sperm samples were excluded on this basis.

\subsubsection{Data analyses}

\subsubsection{Population genetic parameters}

We tested for Hardy-Weinberg proportions (HW) and linkage disequilibrium (LD) using GENEPOP v 4.0.10 (Raymond and Rousset 1995; Rousset 2008). The close relatedness of workers within nests may result in non-independence of genotypes and thus pseudoreplication in HW and LD analyses (Drescher et al. 2007). We corrected for this by using a single randomly selected ant per nest.

We used Genclone v 2.0 (Arnaud-Haond and Belkhir 2007) to enumerate the multilocus genotypes (MLGs) of the ants sampled. To enable easier visualisation of MLGs we grouped and encoded alleles according to their observed distribution among queens, workers and sperm. Alleles that were not found in homozygous queens, but found in male brood, workers or sperm were designated as paternal alleles (i.e., putatively paternally inherited). The other of the pair of alleles found in heterozygous workers (i.e., putatively maternally inherited), which were found in homozygous queens, we designated as maternal alleles. We enumerated allele frequencies using GenAlEx v 6.3 (Peakall and Smouse 2006).

\subsubsection{Population structure}

We used AWclust (Gao and Starmer 2008) to estimate population structure. Unlike other methods (e.g., STRUCTURE: Pritchard et al. 2000), AWclust makes no assumptions about HW proportions or LD, and thus appears appropriate to use when an unusual reproductive mode is possible. In addition, the results enable easier identification of individuals on the resulting tree than STRUCTURE. 


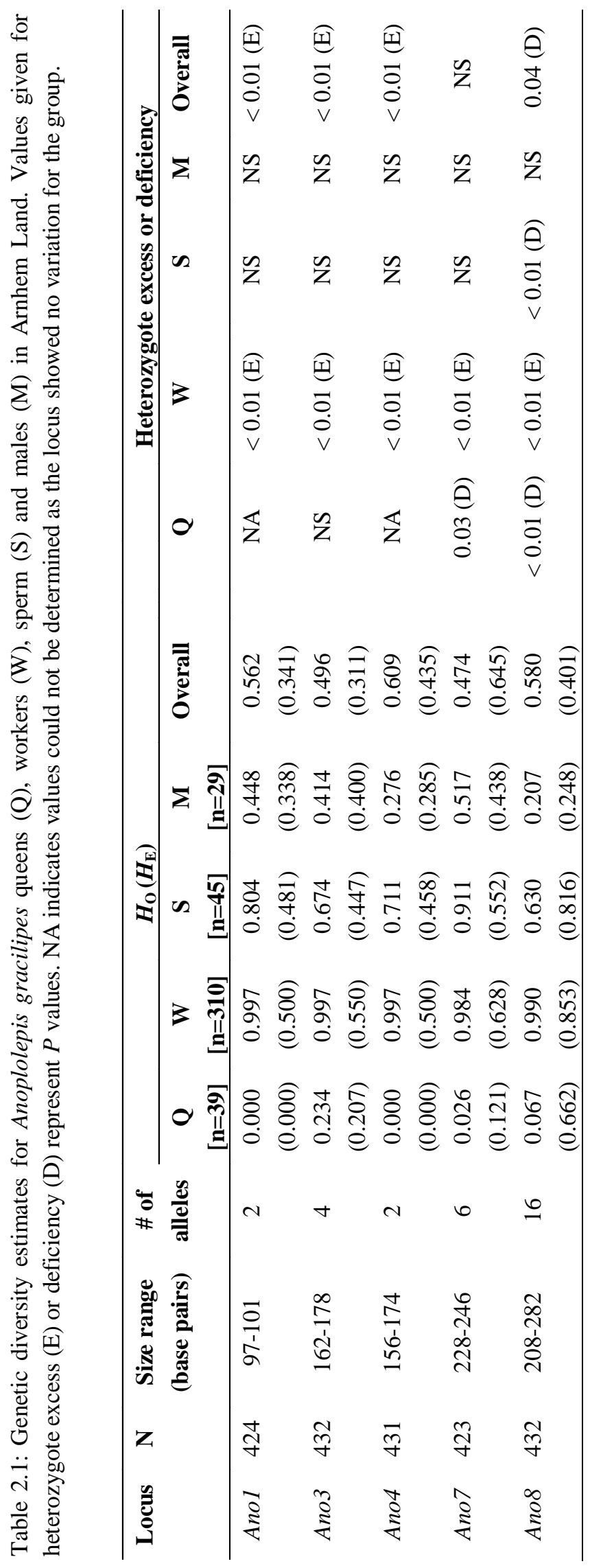




\section{$2.4 \quad$ Results}

\subsubsection{Population genetic parameters}

Microsatellite diversity was consistent with the levels of diversity seen in single populations of A. gracilipes (Drescher et al. 2007; Thomas et al. 2010). Microsatellite genetic diversity among sites was low in two loci (Anol and Ano4), which had the same 2 alleles in all heterozygotes, and one allele in all queens (Table 2.1). The remaining loci (Ano3, Ano7 and Ano8) had four, six and 16 alleles respectively (Table 2.1).

Departures from Hardy-Weinberg (HW) proportions and linkage disequilibrium (LD) were consistent with an unusual reproductive mode. Departures from HW proportions were significant (Table 2.1). Heterozygote deficiency could not be determined for queens for Anol and Ano4 as these loci were fixed at two alleles, but was significant for Ano 7 and Ano8. Heterozygote excess was significant for workers for all loci (Table 2.1). Heterozygote deficiency was significant for sperm for Ano8 (Table 2.1). Linkage disequilibrium could not be estimated for all 10 pairwise locus combinations, because of a lack of genetic variation in Anol and Ano4. Only one comparison was possible for queens, and LD was not significant $(P=1.000)$. For sperm LD was significant in three of 10 pairwise comparisons: Anol and Ano4 $(P=0.002)$; Ano4 and Ano8 $(P=0.011)$; and Anol and Ano3 $(P=0.036)$. For workers LD could only be determined for three of 10 pairwise comparisons and was not significant for any of these comparisons. 


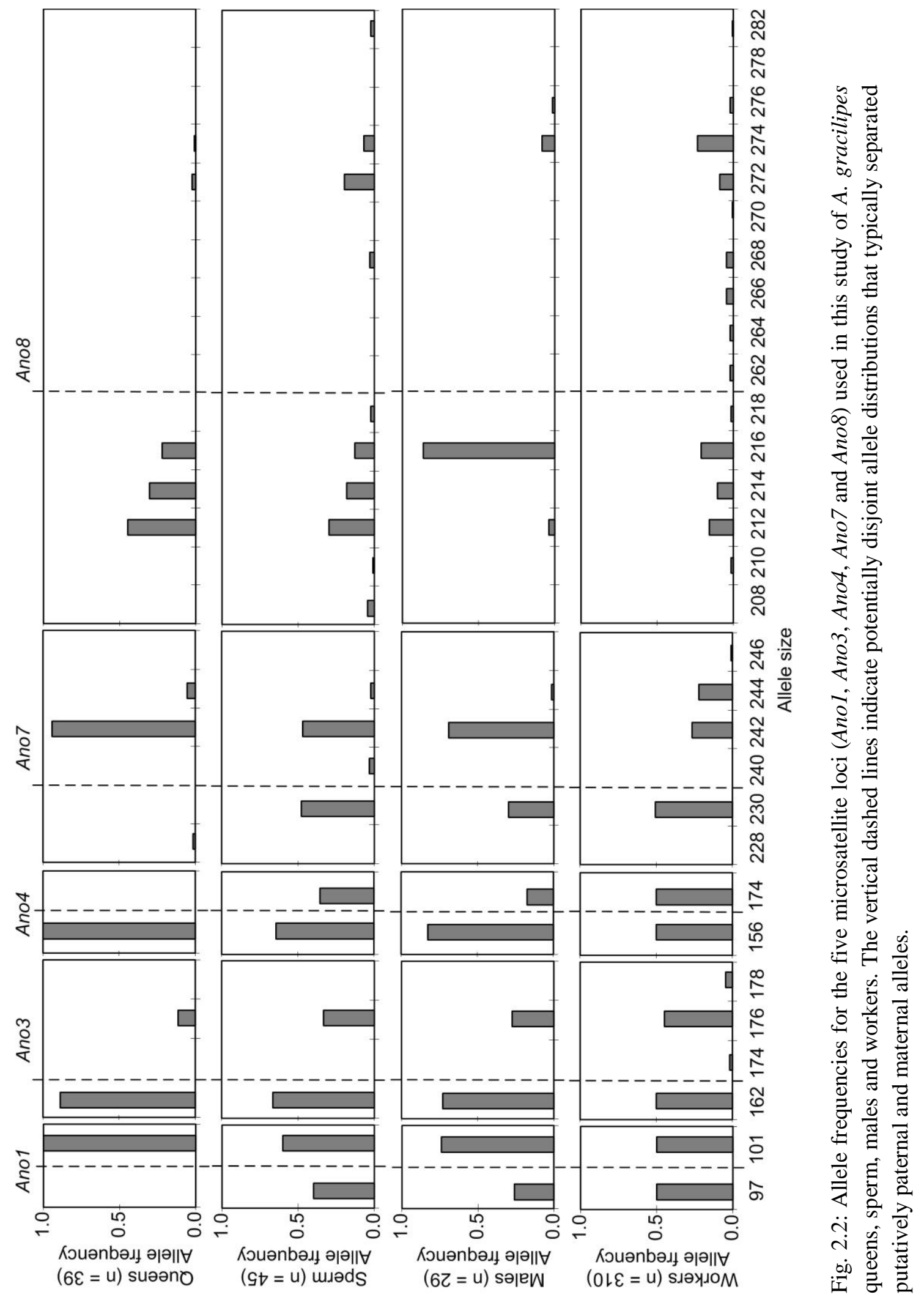


Table 2.2: Multi-locus genotypic patterns among A. gracilipes queens, sperm, males (and male brood) and workers. Alleles have been encoded as letters for ease of interpretation. $A, a$ $=$ Ano1, B, b = Ano3, C, $c=$ Ano4, D, $d=$ Ano7, E, $e=$ Ano8, $-1=$ failed to amplify. Lower case denotes alleles typically found in males, male brood, and workers and only occasionally in queens (putatively paternal). Upper case denotes alleles found in queens, workers and sperm (putatively maternal).

\begin{tabular}{|c|c|c|c|c|c|c|c|c|c|c|c|c|c|}
\hline \multicolumn{10}{|c|}{ Multi-locus genotype } & \multirow{2}{*}{$\frac{\text { Queens }}{25^{1,2,3}}$} & \multirow{2}{*}{$\frac{\text { Sperm }}{1^{1,2,3}}$} & \multirow{2}{*}{$\frac{\text { Males }}{11^{1,2}}$} & \multirow[t]{2}{*}{ Workers } \\
\hline$A$ & $A$ & $B$ & $B$ & $C$ & $C$ & $D$ & $D$ & $E$ & $E$ & & & & \\
\hline$A$ & $A$ & $B$ & $B$ & $C$ & $C$ & $D$ & $D$ & E & $e$ & 2 & & & \\
\hline$A$ & $A$ & $B$ & $B$ & $C$ & $C$ & $d$ & $D$ & $E$ & E & 1 & 5 & & \\
\hline$A$ & $A$ & $B$ & $B$ & $C$ & $c$ & $d$ & $D$ & $E$ & E & & 1 & & \\
\hline$A$ & $A$ & $B$ & $b$ & $C$ & $C$ & $D$ & $D$ & $E$ & $e$ & 1 & & 1 & \\
\hline$A$ & $A$ & $B$ & $b$ & $C$ & $C$ & $d$ & $D$ & $E$ & E & & 1 & & \\
\hline$A$ & $A$ & $B$ & $b$ & $C$ & $C$ & $D$ & $D$ & $E$ & E & 10 & 1 & & 1 \\
\hline$a$ & $a$ & $b$ & $b$ & $c$ & $c$ & $d$ & $d$ & $E$ & $e$ & & & 1 & \\
\hline$a$ & $A$ & $B$ & $B$ & $C$ & $c$ & $d$ & $D$ & $E$ & $e$ & & 5 & & \\
\hline$a$ & $A$ & $B$ & $b$ & $C$ & $C$ & $d$ & $D$ & $E$ & $E$ & & 3 & & \\
\hline$a$ & $A$ & $B$ & $b$ & $C$ & $c$ & $d$ & $d$ & $E$ & $e$ & & 1 & & 4 \\
\hline$a$ & $A$ & $B$ & $b$ & $C$ & $c$ & $d$ & $D$ & $E$ & $E$ & & & 1 & 1 \\
\hline$a$ & $A$ & $B$ & $b$ & $C$ & $C$ & $D$ & $D$ & $E$ & $E$ & & 1 & & \\
\hline$a$ & $A$ & $B$ & $B$ & $C$ & $c$ & $d$ & $D$ & $E$ & $E$ & & 2 & & 1 \\
\hline$a$ & $A$ & $B$ & $b$ & $C$ & $c$ & $d$ & $D$ & $E$ & $e$ & & $23^{4}$ & $34^{4}$ & $303^{4}$ \\
\hline$a$ & $A$ & $B$ & $B$ & $C$ & $C$ & $d$ & $D$ & $E$ & $E$ & & 1 & 1 & \\
\hline-1 & -1 & $B$ & $B$ & $C$ & $C$ & -1 & -1 & $E$ & $E$ & 6 & & & \\
\hline-1 & -1 & $B$ & $B$ & $C$ & $C$ & -1 & -1 & -1 & -1 & 2 & & & \\
\hline$a$ & $A$ & $B$ & $b$ & -1 & -1 & -1 & -1 & $E$ & $E$ & & 1 & & \\
\hline$A$ & $A$ & $B$ & $B$ & $C$ & $C$ & $D$ & $D$ & $e$ & $e$ & & & 1 & \\
\hline$A$ & $A$ & $B$ & $B$ & $C$ & $c$ & $d$ & $D$ & $E$ & $e$ & & & 1 & \\
\hline$a$ & $A$ & $B$ & $B$ & $C$ & $c$ & $D$ & $D$ & $E$ & $e$ & & & 4 & \\
\hline$a$ & $A$ & $B$ & $B$ & $C$ & $c$ & $D$ & $D$ & $E$ & $E$ & & & 2 & \\
\hline$a$ & $A$ & $B$ & $b$ & $C$ & $c$ & $D$ & $D$ & $E$ & $e$ & & & 1 & \\
\hline$a$ & $A$ & $B$ & $b$ & $C$ & $c$ & $D$ & $D$ & $E$ & $E$ & & & 1 & \\
\hline$A$ & $A$ & $B$ & $b$ & $C$ & $c$ & $d$ & $D$ & $E$ & $e$ & & & 1 & \\
\hline
\end{tabular}

\footnotetext{
${ }^{1}$ Homozygous multi-locus genotypes with maternal alleles at all loci

${ }^{2}$ Putative haploid male multi-locus genotypes. Heterozygous genotypes are assumed to be diploid

${ }^{3}$ Putative haploid sperm multi-locus genotypes with alleles that differed from the genotype of the queen from which they were extracted

${ }^{4}$ Heterozygous multi-locus genotypes with paternal and maternal alleles at all loci
} 


\section{Chapter 2}

\subsubsection{Genotypic patterns}

If the reproduction mode of $A$. gracilipes was a DL GCD system we would have expected to see genotypic patterns among workers that reflected a combination of queen and male genotypes, or complementary queen genotypes. Genotype and allele frequencies did differ considerably among queens, workers, sperm, and male brood. Queens were typically homozygous at most loci (Fig. 2.2; Table 2.2), and shared the same (putatively maternal) allele at each of the two loci (Fig. 2.2). Queens were also never heterozygous for different maternal alleles. Queen MLGs from a single nest were not always identical. In 10 of the 12 nests for which two or more queens were genotyped, queen genotypes differed (e.g., Table 2.3). No queens were homozygous for putatively paternal alleles at any locus, which indicates a single lineage of queens is present in Arnhem Land.

In almost all cases workers harboured both a paternal and maternal allele at all loci. Paternal alleles that were found in workers were rarely found in queens at loci Ano3, Ano7 and Ano8. In all nests except BA8 and BY multiple worker MLGs were present, and often worker genotypes could not be assigned back to a queen and / or sperm from the same nest, which suggests that worker exchange among nests is common.

We found no evidence of males belonging to a lineage that corresponded with a putatively paternal lineage (i.e., no males had genotypes that would be expected if paternal MLGs were complementary to maternal MLGs [i.e., aabbccddee MLGs, Table 2.2]). Sperm samples displayed genotypic patterns that were similar to workers, typically being heterozygous at all loci, and possessing both maternal and paternal alleles (Fig. 2.2, Table 2.2). More than $60 \%$ of males and $90 \%$ of sperm genotypes were heterozygous. In addition, all heterozygote genotypes showed a bimodal distribution, with putatively paternal and maternal alleles clearly distinct from each other (Fig. 2.2). Eighteen of 46 sperm samples had alleles that did not match the maternal allele of the queen from which they had been dissected in at least one locus, which indicates the genotype of the mother of the males that produced the sperm differed from the queen genotype from which they had been dissected. The genotypic pattern of male brood and adult males was somewhat similar to that of sperm, although more male genotypes were similar to queen genotypes (Table 2.2). 
Table 2.3: Examples of genotypes of males, queens, workers and sperm for three $A$. gracilipes nests sampled in Arnhem Land, indicative of the genotypic patterns found among nests.

\begin{tabular}{|c|c|c|c|c|c|c|c|c|c|c|c|}
\hline Site & Type & & no1 & & no3 & & 04 & & no7 & & $n 08$ \\
\hline $\mathrm{SC} 3$ & Male & 101 & 101 & 162 & 162 & 156 & 156 & 242 & 242 & 212 & 212 \\
\hline $\mathrm{SC} 3$ & Queen & 101 & 101 & 162 & 162 & 156 & 156 & 242 & 242 & 216 & 216 \\
\hline $\mathrm{SC} 3$ & Queen & 101 & 101 & 162 & 176 & 156 & 156 & 242 & 242 & 216 & 216 \\
\hline $\mathrm{SC} 3$ & Queen & 101 & 101 & 162 & 176 & 156 & 156 & 242 & 242 & 216 & 216 \\
\hline SC3 & Queen & 101 & 101 & 162 & 162 & 156 & 156 & 242 & 242 & 216 & 216 \\
\hline $\mathrm{SC} 3$ & Queen & 101 & 101 & 162 & 176 & 156 & 156 & 242 & 242 & 216 & 216 \\
\hline SC3 & Sperm & 101 & 101 & 162 & 162 & 156 & 156 & 230 & 242 & 216 & 216 \\
\hline SC3 & Sperm & 101 & 101 & 162 & 176 & 156 & 156 & 230 & 242 & 216 & 216 \\
\hline SC3 & Sperm & 97 & 101 & 162 & 176 & 156 & 156 & 230 & 242 & 216 & 216 \\
\hline SC3 & Sperm & 97 & 101 & 162 & 176 & 156 & 156 & 242 & 242 & 226 & 226 \\
\hline $\mathrm{SC} 3$ & Worker & 97 & 101 & 162 & 176 & 156 & 174 & 230 & 242 & 216 & 274 \\
\hline SC3 & Worker & 97 & 101 & 162 & 176 & 156 & 174 & 230 & 242 & 212 & 274 \\
\hline SC3 & Worker & 97 & 101 & 162 & 176 & 156 & 174 & 230 & 242 & 216 & 274 \\
\hline SC3 & Worker & 97 & 101 & 162 & 176 & 156 & 174 & 230 & 242 & 212 & 274 \\
\hline $\mathrm{SC} 3$ & Worker & 97 & 101 & 162 & 176 & 156 & 174 & 230 & 242 & 212 & 274 \\
\hline YI2 & Male & 97 & 101 & 162 & 176 & 156 & 174 & 230 & 242 & 216 & 276 \\
\hline YI2 & Male & 97 & 101 & 162 & 162 & 156 & 156 & 230 & 242 & 216 & 216 \\
\hline YI2 & Male & 97 & 101 & 162 & 176 & 156 & 174 & 230 & 244 & 216 & 216 \\
\hline YI2 & Queen & 101 & 101 & 162 & 162 & 156 & 156 & 242 & 242 & 214 & 214 \\
\hline YI2 & Sperm & 97 & 101 & 162 & 176 & 156 & 174 & 230 & 242 & 214 & 274 \\
\hline YI2 & Worker & 97 & 101 & 162 & 176 & 156 & 174 & 230 & 242 & 212 & 274 \\
\hline YI2 & Worker & 97 & 101 & 162 & 176 & 156 & 174 & 230 & 242 & 212 & 274 \\
\hline YI2 & Worker & 97 & 101 & 162 & 176 & 156 & 174 & 230 & 242 & 214 & 274 \\
\hline YI2 & Worker & 97 & 101 & 162 & 176 & 156 & 174 & 230 & 242 & 216 & 274 \\
\hline MM3 & Queen & 101 & 101 & 162 & 162 & 156 & 156 & 242 & 242 & 212 & 212 \\
\hline MM3 & Queen & 101 & 101 & 162 & 162 & 156 & 156 & 244 & 244 & 212 & 212 \\
\hline MM3 & Queen & 101 & 101 & 162 & 162 & 156 & 156 & 242 & 242 & 212 & 212 \\
\hline MM3 & Sperm & 101 & 101 & 162 & 162 & 156 & 156 & 230 & 242 & 212 & 212 \\
\hline MM3 & Sperm & 97 & 101 & 162 & 176 & 156 & 174 & 230 & 244 & 212 & 272 \\
\hline MM3 & Sperm & 101 & 101 & 162 & 162 & 156 & 174 & 230 & 242 & 212 & 212 \\
\hline MM3 & Worker & 97 & 101 & 162 & 176 & 156 & 174 & 230 & 244 & 216 & 274 \\
\hline MM3 & Worker & 97 & 101 & 162 & 176 & 156 & 174 & 230 & 242 & 212 & 272 \\
\hline MM3 & Worker & 97 & 101 & 162 & 176 & 156 & 174 & 230 & 244 & 212 & 276 \\
\hline
\end{tabular}




\section{Chapter 2}

\subsubsection{Population structure}

The population structure among queens, workers, males and sperm was consistent with the genotypic patterns we observed. The population was divided into two main clusters: one consisting primarily of workers, and the other primarily of queens and males (Fig. 2.3). However, sperm and males were found in both clusters. Samples within sites did not cluster together (Fig. 2.4).

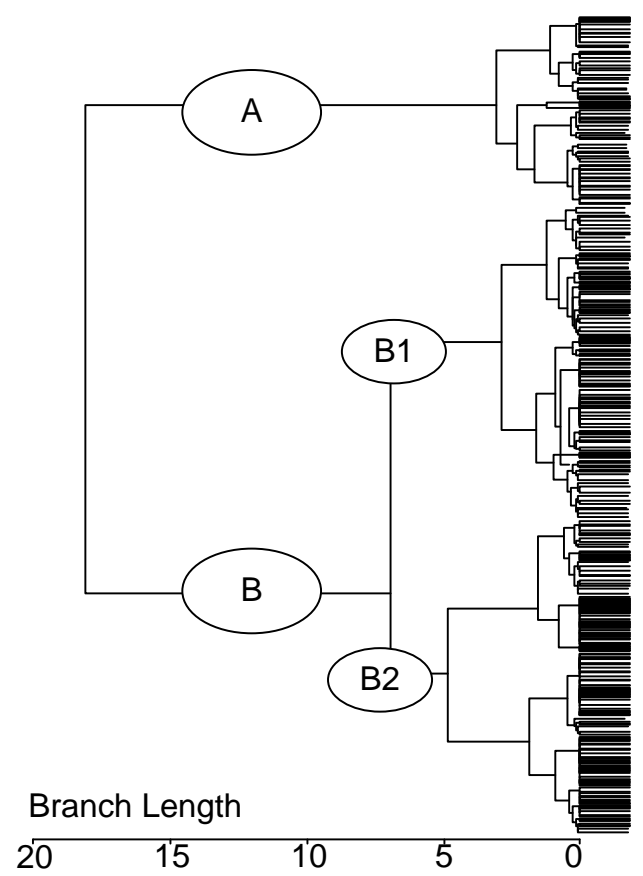

Fig. 2.3: Population structure of A. gracilipes queens, workers, sperm and males in Arnhem Land. The hierarchical plot was based on Allele Sharing Distance calculated with Ward's minimum variance algorithm. Queens were found in cluster A, and workers were found in cluster B. Sperm and males were found in both clusters. Details of cluster membership are provided in Fig 2.4. 


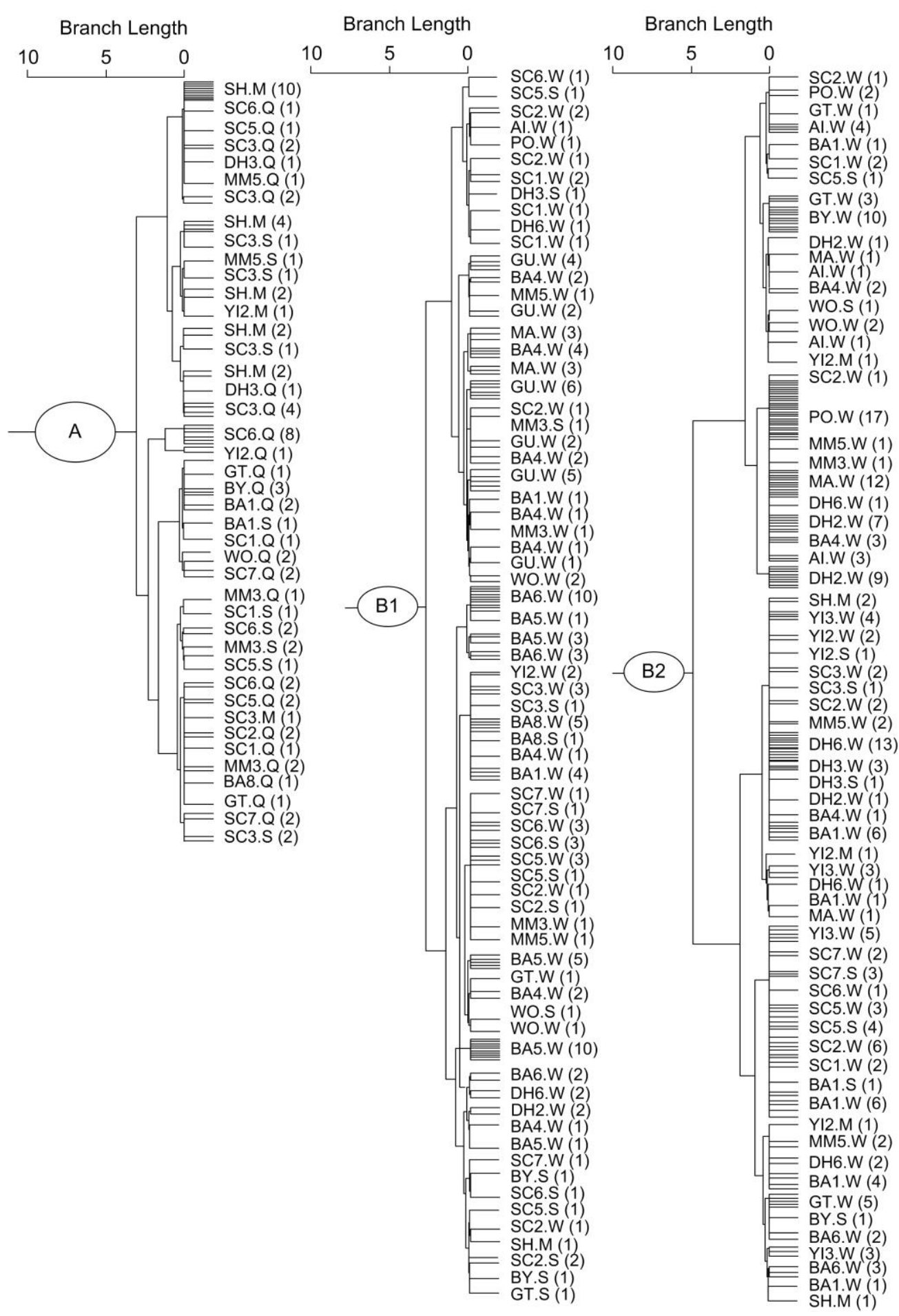

Fig. 2.4: Detailed population structure of A. gracilipes queens (Q), workers (W), sperm (S) and males (M) in Arnhem Land. The hierarchical plot was based on Allele Sharing Distance calculated with Ward's minimum variance algorithm, and shows details of cluster membership. Queens were found in cluster A, and workers were found in cluster B. Sperm and males were found in both clusters. The numbers in brackets summarise the number of ants on that branch for each caste within a site. Site codes refer to the locations in Fig. 2.1. 


\section{Chapter 2}

\subsubsection{Worker reproduction}

Dissections of workers indicated they were unlikely to be able to reproduce. Our dissections of 470 workers from 13 nests (mean $=36 \pm 5 \mathrm{SE}$ ) found ovaries in $2 \%$ to $15 \%$ of workers from seven nests (Fig. 2.5). No spermathecae were found, which indicates the workers did not mate with males. Vitellogenesis, yolky oocytes, and yellow bodies that are characteristic of fertile eggs (Peeters 1987) were not apparent.

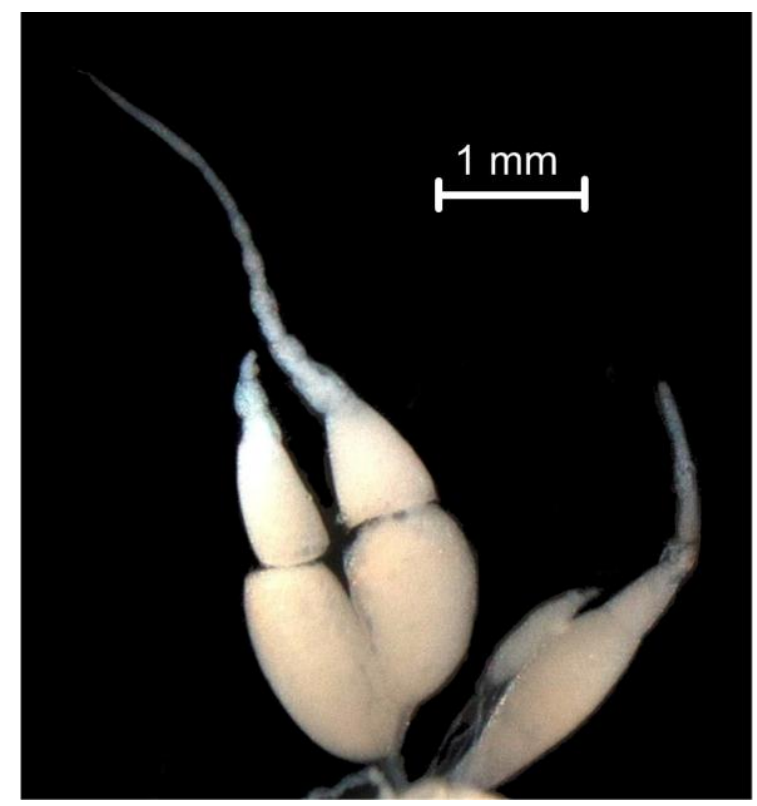

Fig. 2.5: Gaster dissection of A. gracilipes worker, showing ovarioles with no evidence of yolky oocyte development or yellow bodies. 


\subsection{Discussion}

Recently it has been suggested that the ecological success of some invasive ants may be facilitated by clonal reproduction (Foucaud et al. 2009; Kellner and Heinze 2010; Pearcy et al. 2011). By contrast, populations of ant species with DL GCD could have reduced chances of invasion success if one lineage is under-represented. Although it has been suggested that $A$. gracilipes reproduction could involve DL GCD (Drescher et al. 2007), we found clear evidence to reject this hypothesis in Arnhem Land. We found evidence of only one lineage of queens, to which most male genotypes also belonged. Although we found no physiological or genetic evidence of asexual worker reproduction, we cannot entirely discount this possibility, as workers may reproduce seasonally during times we did not assess.

\subsubsection{Dependent lineage genetic caste determination}

We found no evidence of multiple lineages of reproductives to support DL GCD. Our sampling covered a very broad area, so that it was likely that we would have discovered at least a few examples of multiple lineages if they existed. If heterozygous workers were produced through DL GCD, we would have expected our data to show three overlapping populations, with homozygous queen genotypes matching each of the alleles found in the heterozygous worker genotypes. These features were not apparent in the population structure of $A$. gracilipes in Arnhem Land. The absence of any queens homozygous for the putatively paternal alleles in workers, despite extensive sampling, strongly suggests that the reproductive mode of $A$. gracilipes does not involve DL GCD. However, the divergent genotypic pattern between workers and queens does suggest that caste determination is genetic, and the microsatellite loci we have used might be strongly linked to a caste-determining locus. The disjoint distribution we found among microsatellite alleles are possible indicators of ancient hybridisation events, or clonal reproduction, but also may reflect the mode of microsatellite evolution. 


\section{Chapter 2}

\subsubsection{Potential alternative reproductive modes}

The pattern of heterozygous workers, predominantly homozygous queens and often heterozygous males and sperm found in this study is consistent with the results of other studies of this species (Borneo: Drescher et al. 2007; Christmas Island: Thomas et al. 2010), which suggests that the mode of reproduction of A. gracilipes may not vary throughout the invaded range. However, the actual reproductive mode remains unclear. The consistently observed patterns of fixed heterozygote genotypes among workers suggest a number of possible underlying genetic mechanisms, including the strong linkage of microsatellite loci to a caste-determining locus (Volny and Gordon 2002), or asexual reproduction by unmated workers (Balloux et al. 2003). Queen genotypes that are primarily homozygous may also indicate asexual reproduction among queens (Simon et al. 2003; Pearcy et al. 2006), albeit by a different mechanism to workers.

\subsubsection{Asexual production of workers}

Our dissections of workers provided little support for worker reproduction. We found ovaries in a minority of $A$. gracilipes workers, but no spermathecae, which indicates that A. gracilipes workers are not capable of sexual reproduction. The presence of ovaries in workers is not sufficient evidence for asexual reproduction because workers in many ant genera produce unfertilised trophic eggs that do not develop (Hölldobler and Wilson 1990), or eggs that develop into haploid males (Bourke and Franks 1995). Despite the presence of ovaries, there were no oocytes or evidence that viable eggs had been laid. However, workers may only reproduce when they are isolated for long periods from the queen (Bourke 1988). Thus, if the ovaries were functional, worker reproduction is either rare, or may occur seasonally differing to our collection time, or only when workers are isolated from queens.

Consistent with the morphological evidence, asexual worker reproduction was also not supported by the genotypic patterns observed. The two possible forms of asexual production of female castes in ants (thelytoky) are ameiotic (apomixis) and meiotic (automixis). Apomixis results in offspring genotypes that are identical to the parent (barring mutations and gene conversions), and overall levels of heterozygosity in the 
offspring are maintained (Suomalainen et al. 1987). By contrast, automixis results in a steep loss of heterozygosity over a few generations (Pearcy et al. 2006). Thus, if reproduction by $A$. gracilipes workers occurs by automixis, it must be very rare as we found only five workers with homozygous loci, and only one locus at most was homozygous in any single worker. The alternative mode of clonality, apomixis, would appear to be a more likely mode of parthenogenesis among A. gracilipes workers (if it occurs) because apomixis retains heterozygosity and offspring are identical to their mother. Low recombination rates, reflected in departures from HW expectations and significant LD, are typical indicators of asexual reproduction by apomixis (Balloux et al. 2003). Although we found departures from HW among workers, the degree of LD was not clearly resolved. In summary, there appear to be too many uncertainties to conclude that workers reproduce asexually. The disjoint bimodal allele distributions among heterozygous workers, together with the variability of loci Ano3, Ano7 and Ano8 could equally be interpreted as owing to sexual reproduction between queens and males with complementary alleles.

\subsubsection{Asexual production of queens}

Queen genotypes were almost exclusively homozygous with significant heterozygote deficiency, a pattern consistent with asexual reproduction via automixis (Simon et al. 2003; Pearcy et al. 2006). Automixis causes a rapid loss of heterozygosity over a few generations, but loci distant from the centromere retain heterozygosity (Suomalainen et al. 1987; Pearcy et al. 2006). We found heterozygote genotypes at the Ano3, Ano7 or Ano8 loci in 14 of 39 queens. The polymorphism in these loci, and occasional heterozygote genotypes, thus suggests that if automixis was occurring, these loci might undergo recombination. If workers reproduced asexually, the genotypic patterns suggest it would most likely be via apomixis. Thus, it is problematic to interpret how a system would evolve with different modes of asexuality between workers and queens. It seems simpler to conclude that if one of these castes is produced asexually, it is likely the other is not. 


\section{Chapter 2}

\subsubsection{The enigma of diploid males}

In haplo-diploid species, diploid males are diagnostic of complementary sex determination (CSD) (van Wilgenburg et al. 2006). Under CSD, individuals that are heterozygous at one or more sex loci develop into females, while individuals that are haploid or homozygous at one or more sex loci develop into males (Crozier 1971). Diploid males arise owing to a loss of variation at a CSD locus, are an indicator of inbreeding, are typically inviable or sterile, and are therefore rare (Hedrick and Parker 1997; Zayed and Packer 2005). However, a number of recent studies have found that diploid male Hymenopterans (including ants) can mate and produce viable offspring, although workers are triploid as a result (e.g., Liebert et al. 2005; Cournault and Aron 2009). If this was the case for A. gracilipes, we would perhaps expect to have found genotypes that indicated triploid workers. However, if matched matings occur, the maternal allele of the queen may mask the maternal allele of the diploid male and triploid worker genotypes would not be detectable.

The existence of heterozygous sperm genotypes in our results may not reflect diploid males. An alternative explanation for multiple sperm genotypes from a single queen is the gametes are haploid and that multiple matings occur. This pattern is similar to that seen in DL GCD, where queens mate once with a male of their own lineage and once with a male of the second lineage (Helms Cahan et al. 2002; Volny and Gordon 2002). While A. gracilipes may not require a second lineage, both paternal and maternal alleles are required to produce queens, workers and males, and caste fate may be controlled by a caste-determining locus. While sperm genotypes may be haploid, and queens may be mated to multiple males, this does not explain the presence of diploid male genotypes, or the origin of paternal alleles in workers and diploid males.

Another explanation for the presence of diploid male genotypes is that males may be mosaics of both maternal and paternal cells. Such sex mosaics (gynandromorphs) have been reported for more than 40 ant species among 22 genera (Jones and Phillips 1985), and matings involving mosaics can produce viable non-mosaic offspring (Yoshizawa et al. 2009). Importantly, sex mosaicism is known occur in species with clonal production of males and females (Dobata et al. 2012). Anoplolepis gracilipes males could thus be 
haploid but with cells from both paternal and maternal sources. These haploid males could produce sperm with alleles inherited from either the maternal cells or from the paternal cells. The production of viable offspring, and their caste, could be determined by a combination of male and female alleles. The occurrence of sex mosaics in A. gracilipes, together with caste determination based on the allele combination inherited may provide an explanation for the presence of male and sperm genotypes in both worker and queen clusters.

\subsection{Conclusion}

The range of reproductive systems among Hymenopterans is likely to be far more varied than currently known (Keller 2007; Heinze 2008), and despite the reproductive mode of A. gracilipes remaining unresolved, it is clear that when it is determined it will be novel. Of the many uncertainties, the prevalence of diploid males is most problematic. The production of males, and the source and fate of paternal alleles remain enigmatic. However, the resolution of this enigma will also help determine the mode of production of queens and workers. While it is interesting to speculate on the reproductive system based on our data, experimental laboratory nests that allow the control of the parental relationships between individuals are clearly required to understand this unusual reproductive mode. The variation in abundance that we observe in the A. gracilipes population in Arnhem Land appears not to be owing to a skew in lineage-ratio in a DL GCD system, although it does appear that caste determination has a genetic basis. Whether the unusual reproductive mode affects the abundance, fitness and invasion success of this ant remains an intriguing question. 


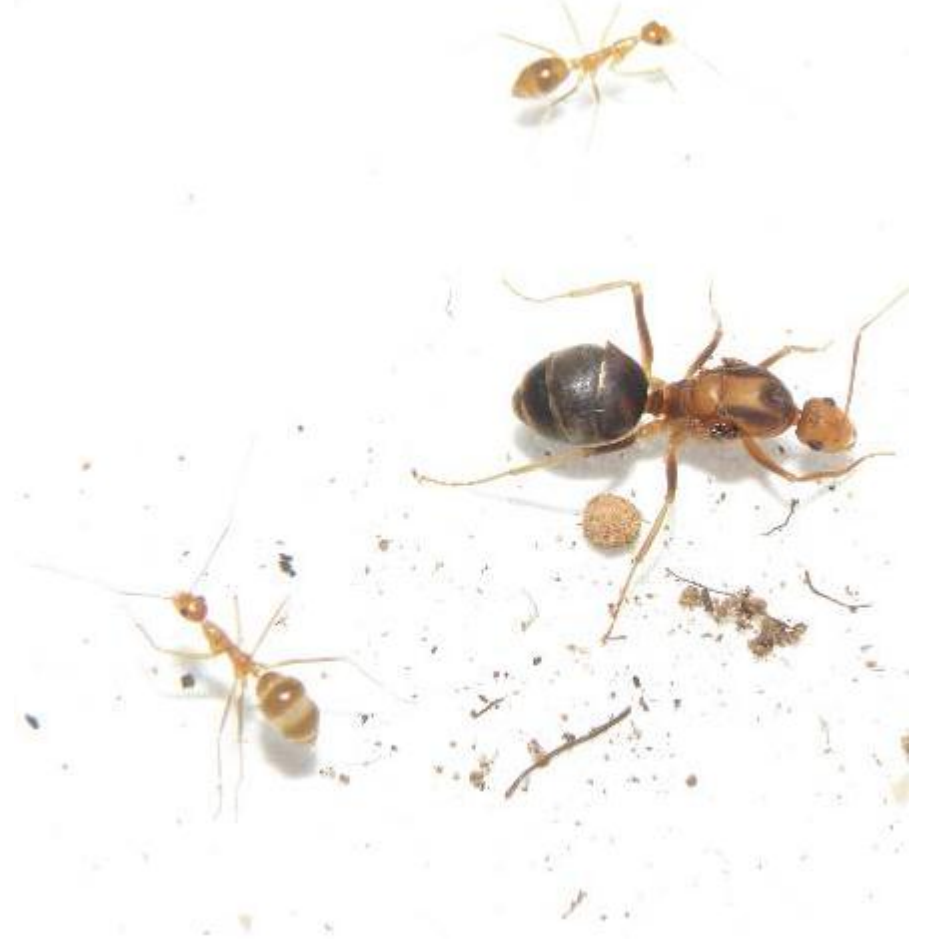




\section{Chapter 3. Recent behavioural and population genetic divergence of an invasive ant in a novel environment}

\subsection{Abstract}

Invasive species frequently exhibit high temporal and spatial variation in abundance. Although ecological aspects undoubtedly affect this variation, genetic factors may also play a part. The invasive unicolonial yellow crazy ant Anoplolepis gracilipes exhibits considerable variation in abundance throughout its extensive distribution in Arnhem Land in Australia's Northern Territory, where it was first detected in the 1980s. First, we aimed to determine if $A$. gracilipes variation in abundance was associated with behavioural and genetic differentiation of the population, and to determine whether one or more introductions occurred. Second, we investigated if the A. gracilipes population was genetically and behaviourally heterogeneous to determine if population divergence has occurred since introduction. Ant abundances were assessed at 13 sites throughout the study region. We used mitochondrial DNA sequences and microsatellite molecular markers to determine population genetic structure, which we correlated with abundance. Behavioural differentiation was assayed using aggression trials and analysed together with genetic data to investigate population divergence. Although we found considerable variation in abundance, we found no association between population structure and differences in abundance. These analyses suggest that A. gracilipes ants in Arnhem Land resulted from a single introduction. The population is not homogeneous, however, as aggression scores varied over both genetic and geographic distance. We also found a positive relationship between genetic and geographic distance. The variation in abundance in the Arnhem Land population of A. gracilipes is clearly not due to invasion by ants from different sources. The genetic and behavioural differentiation we observed is suggestive of incipient genetic and behavioral divergence, which may be expected over time when an invasive species enters in a new environment. 


\section{Chapter 3}

\subsection{Introduction}

The effects of invasive ant species on the recipient community are often densitydependent; therefore, elucidating the causes of variation in abundance is central to understanding invasive potential. The negative ecological effects of invasive ants on the invaded community typically increase in severity as the abundance of the invader increases (e.g., Macom and Porter 1996; Ross et al. 1996; O'Dowd et al. 2003; Le Breton et al. 2005; Abbott et al. 2007; Krushelnycky and Gillespie 2008; McNatty et al. 2009). Although ecological aspects undoubtedly affect variation in abundance, genetic factors may also contribute (e.g., Abbott et al. 2007). For example, genetic and behavioural differences among yellow crazy ant (Anoplolepis gracilipes) populations among islands of the Tokelau archipelago were associated with differences in population densities. More recently invading populations were genetically and behaviourally differentiated from populations that had been in Tokelau longer, and had substantially higher densities (Abbott et al. 2007).

The high population densities attained by invasive ants is often associated with unicoloniality. Although not all unicolonial ants are invasive, the ants listed among the 100 most damaging invasive species (Lowe et al. 2000) are unicolonial (Helanterä et al. 2009). Unicolonial ant species form supercolonies that exhibit behavioural and genetic differentiation between supercolonies, but no genetic or behavioural differentiation (i.e., aggression) within individual supercolonies (Pedersen et al. 2006). At the most extreme, individual supercolonies of the Argentine ant (Linepithema humile) that span different continents are entirely behaviourally, genetically and chemically undifferentiated, despite over 100 years of separation (van Wilgenburg et al. 2010). Not all Argentine ant supercolonies are undifferentiated, however. Ants from the Corsican supercolony are chemically and behaviourally different from their source supercolony on the European mainland, despite only 60 years of separation. The genetic relationships between the two colonies have not been investigated, however (Blight et al. 2010). 
Populations of A. gracilipes present as mosaics of genetically and behaviourally differentiated supercolonies (Abbott et al. 2007; Drescher et al. 2007; Drescher et al. 2010). In fact, Drescher et al. (2010) suggested that the divergence between A. gracilipes supercolonies in Borneo may have resulted in incipient speciation. Although Drescher et al. (2010) focussed on divergence between, rather than within supercolonies, they extended their discussion to divergence within colonies that may occur in species where mating is intranidal (within the nest). This scenario entails fragmentation of the colony followed by spatial segregation. Isolation would result in population divergence through genetic drift, and the homogenising effects of gene flow would be eliminated as mating is locally restricted. As the origin of supercolonies may be a developmental process rather than an evolutionary one (Helanterä et al. 2009), the breakdown of individual supercolonies may occur in an observable timeframe, and be more common than has been reported to date.

Populations of A. gracilipes vary in size and density (Lewis et al. 1976; Hill et al. 2003; Abbott 2005; Abbott et al. 2007; Drescher et al. 2011). The native range of this ant is undetermined (Wetterer 2005), so its natural patterns of abundance, colony structure, and influence on the native ant community outside the invaded range are not known. It is clear, however, that in the invaded range its population densities, behaviour, colony structure, and ecological effects vary (Lewis et al. 1976; Hill et al. 2003; Abbott 2005; Abbott et al. 2007; Drescher et al. 2007; Drescher et al. 2011). Although habitat and ecological interactions fundamentally influence differences in abundance (O'Dowd et al. 2003), genetic differences may also be a contributing factor (Abbott et al. 2007). Understanding of the links between population density, behaviour and genetic population structure in A. gracilipes would improve our knowledge of its invasive potential, and the nature of unicoloniality in this species.

In Arnhem Land in Australia, A. gracilipes has a fragmented distribution over a wide region, and also exhibits varying nest and population densities. Unlike A. gracilipes invasions in Samoa (Savage et al. 2009) and on Christmas Island (O'Dowd et al. 2003), carbohydrate resources are not an obvious driver of differences in abundance (authors' unpublished data), nor are there clear associations with anthropogenic disturbance (Hoffmann and Saul 2010). Our study focuses on the relationships between abundance 


\section{Chapter 3}

and population differentiation of $A$. gracilipes in Arnhem Land. First, we test the hypothesis that spatially segregated populations with different densities are also behaviourally and genetically differentiated. Second, we investigate if Arnhem Land's A. gracilipes colony or colonies are genetically and behaviourally heterogeneous to test the hypothesis that divergence within colonies has occurred, and if divergence is related to patterns of abundance.

\subsection{Methods}

\subsubsection{Study site}

Arnhem Land is located in the Australia's Northern Territory. The regional climate is tropical monsoonal with temperatures ranging between 17 and $33^{\circ} \mathrm{C}$ throughout the year, and seasonal rainfall (December to July) of approximately $1200 \mathrm{~mm}$. The vegetation is primarily savanna woodland. Although it is not known when A. gracilipes arrived in the region, it was first detected in 1982 (Majer 1984). Thorough surveys in 2009 found the ant to be patchily distributed throughout $16,000 \mathrm{~km}^{2}$ in Arnhem Land (Fig. 3.1).

Sites throughout the geographical range of A. gracilipes in Arnhem Land were chosen in October 2007 based on our preliminary observations of differing population densities and abundance, and ecological similarity (a canopy dominated by Eucalyptus tetrodonta, an understorey primarily of Acacia spp., grasses and leaf litter, and similar drainage and topography). These preliminary observations were achieved through extensive surveys of the region, where all occurrences of the ant were recorded (Fig. 3.1). 


\subsubsection{Sampling}

Approximately 30 workers from a single nest at each of seven sites were collected in October 2007 for genetic analyses (Fig. 3.1). Based on these earlier observations, between July 6 and 172009 we made additional collections of 100 - 200 workers from a single nest at 13 sites for genetic and behavioural analyses (Fig. 3.1). We recorded the location of each site using a GPS (Garmin), and measured the distance between sites using ArcMap v 9.3 (Esri). To test for genetic and behavioural differences relative to population densities, we measured the abundance of A. gracilipes at each site based on forager activity.

Counts of ant abundance were conducted before 10am when ambient temperatures do not prevent $A$. gracilipes from foraging. The site level abundance of $A$. gracilipes was assessed using card counts, which measure forager activity using a count of the number of ants crossing a white $10 \times 10 \mathrm{~cm}$ laminated card (Green et al. 2004). At each site we measured A. gracilipes activity at 11 stations spaced $5 \mathrm{~m}$ apart on $50 \mathrm{~m}$ transects, with three replicate transects spaced $10 \mathrm{~m}$ apart. At each station the vegetation was scraped away to reveal bare ground. A $20 \times 20 \mathrm{~cm}$ laminated card, divided into four quadrants, was placed on the ground and observed for $20 \mathrm{~s}$. The first quadrant crossed by an $A$. gracilipes worker was used as the focal quadrant, and for the following $30 \mathrm{~s}$ all ants crossing that quadrant were counted. If no ants were observed on the card during the first $20 \mathrm{~s}$, the first quadrant visited by an ant in the following $30 \mathrm{~s}$ was used. An individual walking across the quadrant multiple times was counted only once. Counts at all stations within a transect were summed, and the mean value of the three replicate transects was used as an index of relative abundance between sites. We also calculated the coefficient of variation $(\mathrm{CV})$ of card counts as a measure of variability in abundance within and among sites. 


\section{Chapter 3}

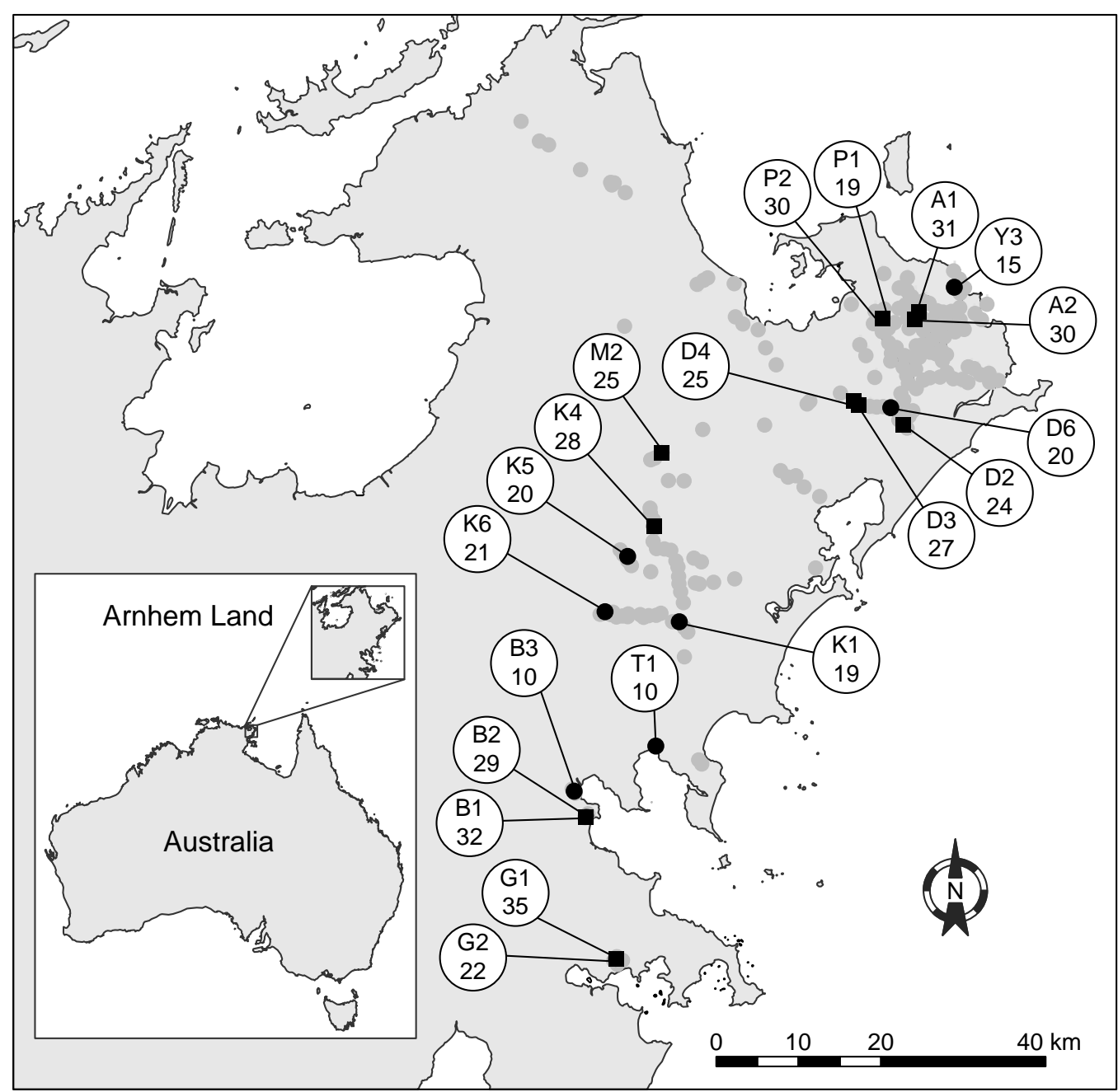

Fig. 3.1: Anoplolepis gracilipes distribution and sample sites in Arnhem Land in the Northern Territory, Australia. Pale circles $(O)$ represent the known extent of the invasion at the time of sampling. Black circles $(\mathbf{O})$ are sites sampled in October 2007 for analysis of population structure. Black squares ( $\boldsymbol{\square}$ ) represent the 13 sites sampled in July 2009 for genetic analysis and behavioural assays. Numbers below site codes are the number of ants genotyped for the site.

\subsubsection{Genetic analyses}

Ants collected for genetic analysis were stored in $95 \%$ ethanol at $4{ }^{\circ} \mathrm{C}$. We extracted DNA using a modified Chelex protocol (Sepp et al. 1994). Individual workers were placed in microcentrifuge tubes, ground with sterile plastic pestles, and $150 \mu 1$ of a $10 \%$ w/v Chelex-100 resin solution was added. The tubes were centrifuged briefly, boiled for 
15 minutes in a water bath, chilled on ice for five minutes, and centrifuged at $15,000 \times g$ for 15 minutes at $4^{\circ} \mathrm{C}$. The supernatant containing DNA was stored at $4{ }^{\circ} \mathrm{C}$.

We used mitochondrial markers to determine if multiple haplotypes were present, targeting the COI (cytochrome oxidase I) region. We sequenced one ant per site with the exception of Y3 (one ant from each of five nests), G2, K4, M2, and K1 (one ant from each of two nests). PCR reagents and thermal cycling were the same as microsatellite analysis (see below), but with equal concentrations of only forward and reverse primers (CI-13 and CI-14, Quek et al. 2004), and with 40 cycles using a $40^{\circ} \mathrm{C}$ annealing temperature. Amplified products were purified using ExoSAP-IT (US Biochemicals) and sequenced on a 3730 Genetic Analyser (Applied Biosystems).

Microsatellite markers were used to assess the population genetic structure, genetic distance and relatedness between individuals within and among sites. Workers were genotyped using seven microsatellite loci: Ano1, Ano3, Ano4, Ano5, Ano7, Ano8 and Ano10 (Feldhaar et al. 2006), that are polymorphic in other populations (Drescher et al. 2007; Drescher et al. 2010; Thomas et al. 2010). Each $15 \mu 1$ PCR consisted of $\sim 20 \mathrm{ng}$ template DNA, 10× PCR Buffer, $0.4 \mu \mathrm{g} \mathrm{mL}^{-1}$ of bovine serum albumin (BSA), 1.5 - 2.5 $\mathrm{mM} \mathrm{MgCl} 2,0.2 \mathrm{mM}$ of each dNTP, $0.1 \mathrm{mM}$ forward primer (adapted with M13 tags, Schuelke 2000), $0.4 \mathrm{mM}$ reverse primer, $0.4 \mathrm{mM}$ universal fluorescent dye-labelled M13 primer (Schuelke 2000), and 0.3 U of Taq DNA Polymerase (either Bioline BIOTAQ, Invitrogen Platinum ${ }^{\circledR}$ or New England Biolabs). Fluorescent dyes used were FAM (Ano4, Ano5 and Ano8) and VIC (Ano1, Ano3, Ano7 and Ano10). Polymerase Chain Reactions were performed using the thermal cycling conditions specified by Feldhaar et al. (2006), with modification for M13 primers (Schuelke 2000). Amplified products were analyzed on a 3730 Genetic Analyzer using the LIZ size standard, and visualised and scored using Genemapper v 3.7 (both Applied Biosystems). Between 10 and 35 workers were genotyped per site (mean $\pm \mathrm{SE}=23 \pm 1.58, \mathrm{n}=460$ ). We used Micro-Checker $\mathrm{v}$ 2.2.3 (van Oosterhout et al. 2004) to validate genotyping quality (scoring errors, null alleles, and large allele dropout), and none of these features were observed in our dataset. 


\section{Chapter 3}

Hardy Weinberg (HW) proportions were tested using GENEPOP v 4.0.10 (Raymond and Rousset 1995). High relatedness among nestmates suggests non-independence of genotypes, and leads to pseudoreplication issues in HW analyses. Our relatedness estimates were relatively high (Table 3.1 ), so to be conservative we corrected for pseudoreplication by using a single randomly selected ant per site in our HW tests.

To estimate population structure we used STRUCTURE v 2.3.3 (Pritchard et al. 2000), together with a principal components analysis in PCAGEN (Goudet 2000). Unlike STRUCTURE, PCAGEN makes no assumptions about HW or linkage. We ran 15,000 permutations within PCAGEN to obtain significance values for the variation explained by each axis. STRUCTURE uses a Bayesian Markov Chain Monte Carlo (MCMC) method to assign individuals to one or several genetic clusters $(K)$. We performed 10 STRUCTURE runs for values of $K$ between one and 20, using a model that assumed admixture and correlated allele frequencies, with a burn-in of 100,000 simulations and $1,000,000$ subsequent simulations. The best estimate of $K$ was based on the run with the highest log likelihood, and confirmed using the $\Delta K$ method (Evanno et al. 2005). Using PCAGEN it is only possible to discriminate populations if samples clearly cluster together, and the $\Delta K$ method is able to discriminate two or more genetic clusters only. As we had 20 sites and also wished to determine if this was a single supercolony (i.e., $K=1$ ), to determine the number of clusters we also used FLOCK v 2.0 (Duchesne and Turgeon 2009). We followed the FLOCK software guidelines and used 50 runs, increasing $K$ until plateau lengths were zero for more than four consecutive values of $K$, or plateau lengths were $\geq 6$. In FLOCK analyses, if a plateau length of six is not reached, a single genetic cluster is assumed (i.e., $K=1$ ).

To investigate genetic divergence among sites we tested for genetic isolation by distance (Slatkin 1993) using 10,000 permutations of the relationship between Nei's Distance and geographic distance with a Mantel test in the 'ade4' package (Dray and Dufour 2007) in R (R Development Core Team 2011). We also tested this relationship with Spearman's rank correlation, as the Mantel test has less power and is therefore prone to Type II errors (Legendre and Fortin 2010). 


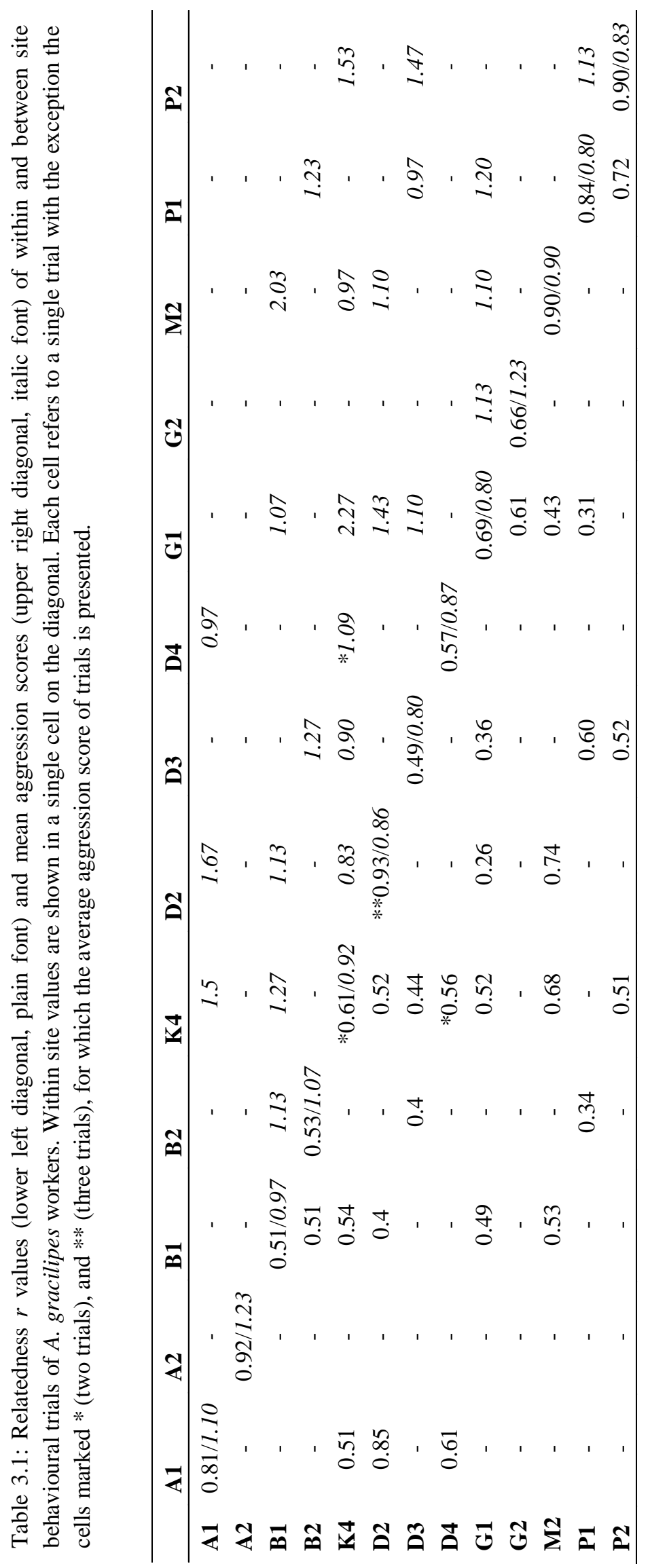




\section{Chapter 3}

To determine whether assignment of individuals to the genetic clusters identified by STRUCTURE had a geographical relationship, we used Spearman's rank correlation. We compared the relationship between proportional cluster assignments with distance along a north south axis. We used the northernmost site as a reference as this is the approximate location of a port, airport and only major human settlement, so could have been the initial invasion point. A Spearman's rank correlation was used to determine if the proportional cluster assignment of individuals was related to abundance. The standard error of mean abundance increased as abundance increased (Table 3.2). To test if there was a positive relationship between variability in abundance and genetic differentiation we used Spearman's rank correlation to compare the coefficient of variation and proportional cluster assignment.

Table 3.2: Abundance and relatedness estimates for A. gracilipes from the 13 sites included in behavioural trials. Abundance, mean $\pm \mathrm{SE}$ and co-efficient of variation $(\mathrm{CV})$, measured using card counts. Values of zero reflect non-detection due to low density, rather than absence; $\mathrm{N}$, number of individuals genotyped; Wang's $r$, mean within nest relatedness $\pm \mathrm{SD}$ (range).

\begin{tabular}{ccccc}
\hline Site & \multicolumn{2}{c}{ Abundance } & N & Wang's $\boldsymbol{r}$ \\
\cline { 2 - 3 } & \multicolumn{1}{c}{ Mean } & $\mathbf{C V}$ & & \\
\hline A1 & $42.3 \pm 9.0$ & 2.8 & 31 & $0.81 \pm 0.13(0.30-1.00)$ \\
A2 & $3.0 \pm 0.3$ & 3.4 & 18 & $0.92 \pm 0.07(0.85-1.00)$ \\
B1 & $38.7 \pm 3.8$ & 1.3 & 32 & $0.51 \pm 0.28(0.24-1.00)$ \\
B2 & $35.0 \pm 3.1$ & 1.2 & 29 & $0.53 \pm 0.26(0.26-1.00)$ \\
K4 & $15.0 \pm 1.8$ & 1.2 & 28 & $0.61 \pm 0.23(0.19-1.00)$ \\
D2 & $17.0 \pm 1.6$ & 1.2 & 24 & $0.93 \pm 0.15(0.74-1.00)$ \\
D3 & $1.0 \pm 0.2$ & 3.2 & 27 & $0.49 \pm 0.30(0.30-1.00)$ \\
D4 & $6.3 \pm 1.0$ & 2.2 & 25 & $0.57 \pm 0.27(0.30-1.00)$ \\
G1 & $3.7 \pm 0.3$ & 2.3 & 35 & $0.69 \pm 0.20(0.30-1.00)$ \\
G2 & $3.3 \pm 0.2$ & 1.9 & 22 & $0.66 \pm 0.21(0.30-1.00)$ \\
M2 & $0.0 \pm 0.0$ & 0.0 & 25 & $0.90 \pm 0.10(0.79-1.00)$ \\
P1 & $0.3 \pm 0.1$ & 5.7 & 19 & $0.84 \pm 0.11(0.30-1.00)$ \\
P2 & $2.3 \pm 0.4$ & 2.8 & 30 & $0.90 \pm 0.07(0.30-1.00)$ \\
\hline
\end{tabular}


Genetic diversity measures (observed and expected heterozygosities, allele frequencies) were calculated using GenAlEx v 6.3 (Peakall and Smouse 2006), and allelic richness was calculated using HP-RARE (Kalinowski 2005). We estimated relatedness using Wang's moment estimator of relatedness $(r)$ implemented in MER v 3.0 (Wang 2002). Wang's $r$ is comparable to other indices of relatedness but is a more reliable measure for biallelic loci, where allele frequencies are 0.5 or sample sizes are small, regardless of the allele frequency distribution, or number of alleles per locus (Wang 2002). Wang's $r$ ranges from $0-1$, although negative values are possible where individuals are unrelated. Relatedness values of 1 indicate identical multilocus genotypes. We conducted pairwise comparisons among all individuals, with 1000 bootstraps over loci, and then pooled individual results for each site. Pairwise genetic distances between sites were estimated using Nei's Distance (Nei and Roychoudhury 1974) calculated in GenAlEx v 6.3 (Peakall and Smouse 2006).

\subsubsection{Behavioural assays}

Behavioural assays were conducted to investigate the relationship between intraspecific aggression and geographic and genetic distance. Ants used in behavioural trials were collected from a single nest at each of 13 sites in July 2009 (Fig. 3.1; Table 3.1). Ants were kept at $\sim 23^{\circ} \mathrm{C}$, with a damp tissue for water but no food. Assays were completed within 72 hours of the ants being collected. We assessed aggression among pairs of sites (Table 3.1), using three replicates of five ants from each site for each trial, and controls of ants from the same site. The use of five ants ensures more consistent results across replicates than assays using a single ant (Roulston et al. 2003). Trials were conducted in a fluon-coated arena of $10 \mathrm{~cm}$ diameter with a removable barrier bisecting the arena. The arena floor was lined with a filter paper disc that was replaced after each replicate. Unmarked ants from two sites were placed into respective sides of the arena. Once ants had settled, after approximately 30 seconds, the divider was removed. A scan based approach was used to assess ant behaviour, checking the arena for 5 - 10 seconds every minute over a 10 minute period and recording the highest interaction score. We conducted 45 aggression trials of three replicates each (Table 3.1) over 22.5 hours of observation. As the trials were conducted over a period of two weeks, trials were repeated for some sites (Table 3.1). 


\section{Chapter 3}

Interactions were scored on a five point scale modified from Suarez et al. (1999): $0=$ "Ignore" was a contact in which neither showed interest; $1=$ "Contact" included antennation; 2 = "Avoidance" was a contact resulting in one or both ants retreating; $3=$ "Aggression" consisted of biting, pulling, mouth gaping, twitching; 4 = "Fighting" included lengthy aggression, carrying, or grappling. Aggression scores between 0 and 2 were considered non-aggressive, and above 2 were considered aggressive. The mean score for each replicate was averaged to give a mean aggression score for each trial. Ants from each site were retained for genetic analyses and were processed as described earlier.

The relationships between aggression scores and geographic or genetic distance were analysed using Spearman's rank correlation implemented in $\mathrm{R}$. We tested the relationships between mean aggression score and geographic distance (kilometres between sites), and genetic distance (Nei's Distance). To test if levels of aggression differed in trials within and between sites, we used Wilcoxon rank sum tests (with MonteCarlo resampling to estimate $P$ values), implemented in the 'coin' package in R (Hothorn et al. 2008). We applied a sequential Bonferroni correction (Rice 1989) across all statistical tests, and report Bonferroni corrected $P$ values. 


\section{$3.4 \quad$ Results}

\subsubsection{Genetic differentiation}

Our population genetic analyses indicated that A. gracilipes in Arnhem Land were derived from a single population, although the population structure was not homogeneous. Mitochondrial analyses of 28 samples found sequences of 533 base pairs of a single haplotype (GenBank Accession JN375978). The STRUCTURE analysis identified two genetic clusters using the $\Delta K$ method, which also had the highest mean log likelihood of -6267.04 (-6390.40 for $K=1$ and -6533.50 for $K=3)$. STRUCTURE detects genetic clusters in the data and assigns individuals to these clusters based on genotypes. If there is strong differentiation, sites and clusters should correspond to each other. No differentiation is detectable if an individual is assigned equally to each cluster. Our results showed that although the clusters were not identically correlated with sites, individuals within a site typically assigned to one cluster more than the other (Fig. 3.2(a), (b)). The clusters identified by STRUCTURE followed an approximate north-south distribution (Fig. 3.2(b)). Individuals from the northern and eastern sites were assigned mostly to a single cluster (white; Fig. 3.2(b)). Individuals from the most southern sites were generally, but not always, assigned more often to the second cluster (grey; Fig. 3.2(b)). The presence of both clusters at all sites is consistent with a recent shared history or contemporary gene flow. A clinal pattern of genetic divergence was suggested by a decline in the proportion of individuals in a site assigning to the white cluster as distance from the northernmost site increased (Spearman's $r_{s}=0.71, S=385.64, P=0.001$ ). 

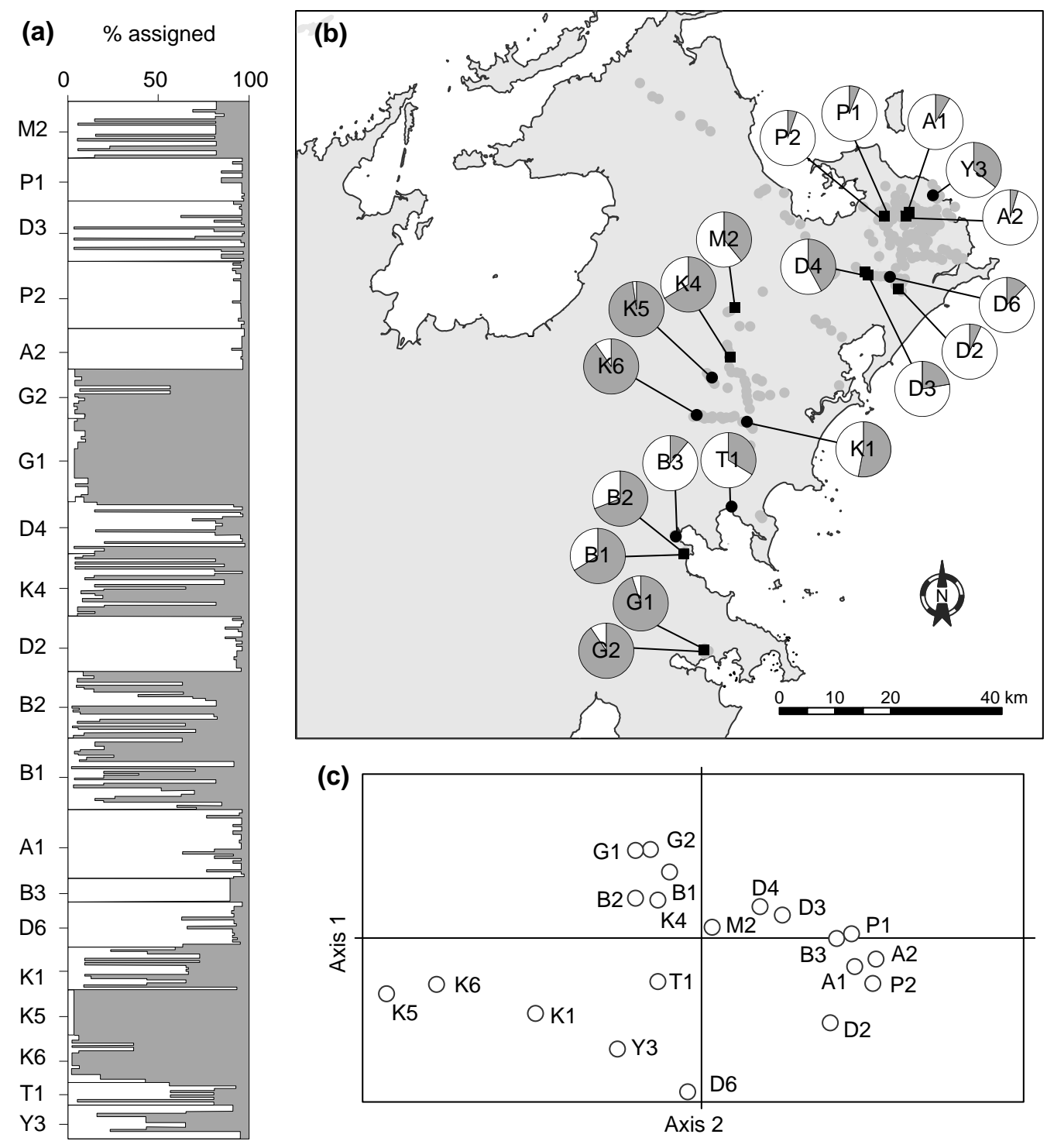

Fig. 3.2: Population genetic structure of A. gracilipes in Arnhem Land. (a) STRUCTURE identified two genetic clusters. Each individual ant is represented by a thin horizontal bar. The amount of each colour (grey or white) indicates the proportion of each inferred cluster to which the individual was assigned. Sites are presented in order of increasing abundance, followed by sites B3, D6, K1, K5, K6, T1, and Y3 for which abundance was not measured. Individuals within a site were typically assigned to both genetic clusters. (b) Geographical representation of the genetic clusters identified by STRUCTURE analysis. Pie graphs show the proportional assignment of individuals from each site to each cluster. The white cluster was more prevalent in the north, while the grey cluster was generally more prevalent in the west and south, a pattern that was significant (Spearman's $r_{s}=0.71, \mathrm{~S}=385.64, P=0.001$ ). (c) Principal components analysis in PCAGEN revealed no strong clustering of sites. PCA axis 1 explains $38 \%$ of the variance $(P=0.073)$, and axis 2 explains $26 \%(P=0.014)$. 
The first two components of the PCAGEN analysis explained 64\% (axis $1=38 \%$ of variance $[P=0.073]$; axis $2=26 \%[P=0.014]$ ) of the variability in the data and showed no definitive clustering of sampling sites (Fig. 3.2(c)). Consistent with this absence of defined population structure, the FLOCK analysis found plateau lengths of zero for values of $2<k<6$, and plateau lengths of 3 and 2 for $k=2$, indicating the best approximation of $K$ is 1 (i.e., a single population). However, we found a significant pattern of genetic isolation by distance between sites included in the behavioural assays (Fig 3.3(a); Mantel test, $r=0.37, P=0.002$; Spearman's $r_{s}=0.57, S=1725.96, P=$ $0.003)$, which indicates genetic divergence within the population. When all sites were included, the relationship was not significant using the Mantel test (Fig. 3.3(b); $r=-0.04$, $P=0.959)$, but was significant when tested using Spearman's rank correlation $\left(r_{s}=0.23\right.$, $S=875217.50, P=0.003)$.

\subsubsection{Genetic diversity}
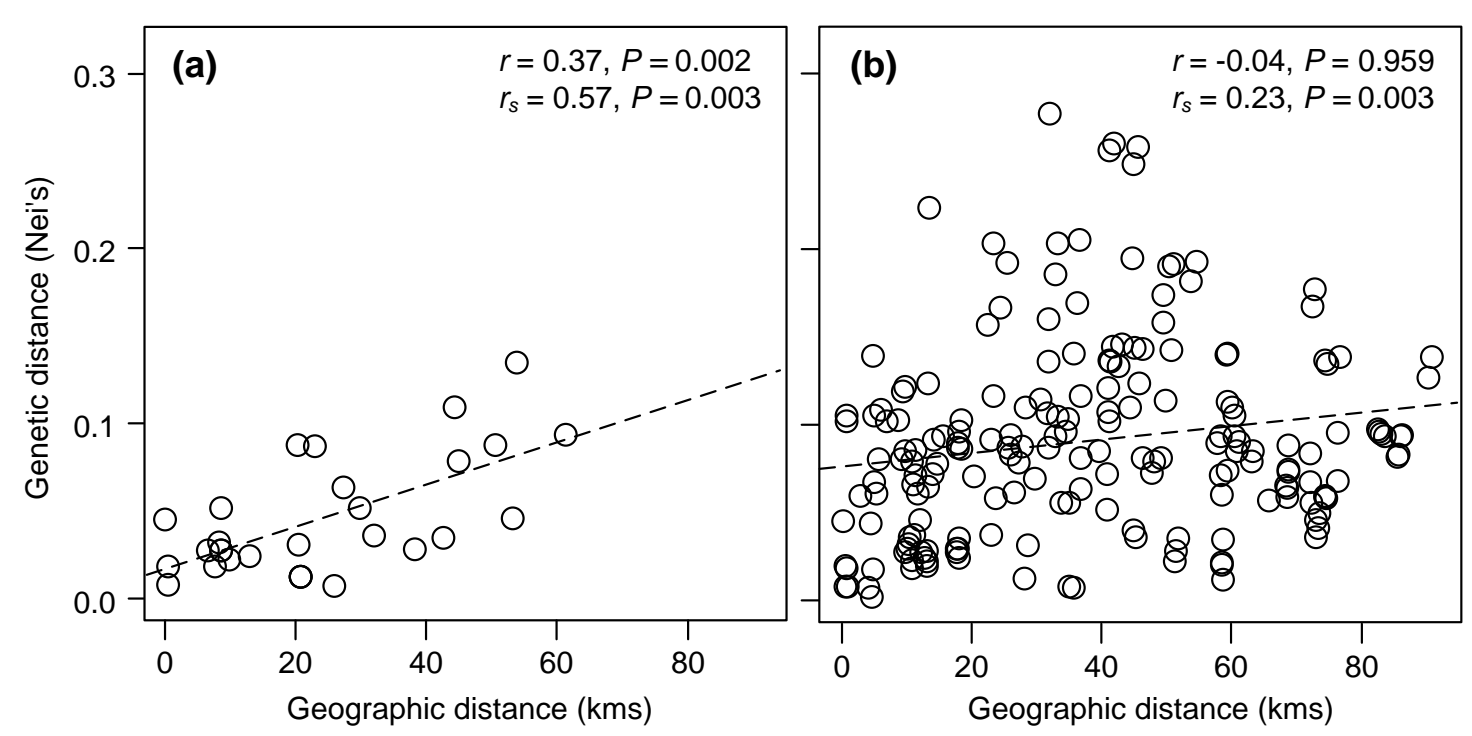

Fig. 3.3: Relationship between Nei's distance and geographical distance among A. gracilipes sites included in behavioural analyses only (a) and all sites (b), with Mantel correlation coefficients $(r)$, Spearman's correlation co-efficients $\left(r_{s}\right)$ and their respective significance values $(P)$. The dashed lines indicate the line of best fit under a linear model. 


\section{Chapter 3}

Estimates of genetic diversity were consistent with a single supercolony. We found low genetic diversity relative to other A. gracilipes studies of multiple supercolonies (Drescher et al. 2007; Thomas et al. 2010) in six of the seven microsatellite loci used. Genetic diversity estimates found between two and 17 alleles per locus, and rarefied allelic richness of between 2.00 and 8.29 alleles per locus per site. All individuals had the same two alleles for the Anol, Ano4, Ano5 and Ano10 loci. All loci except Ano8 (the most diverse locus) exhibited significant heterozygote excess (Table 3.3), consistent with prior findings for this species (Drescher et al. 2007; Thomas et al. 2010).

Relatedness values within and between sites were consistent with a single supercolony. Mean relatedness within sites was $0.73 \pm 0.04$ SE (range: $0.49-0.93$, Table 3.1). Mean relatedness between sites was $0.53 \pm 0.02 \mathrm{SE}$ (range: $0.26-0.93$, Table 3.1). In three cases, relatedness within sites was lower than relatedness between sites (Table 3.1). Within individual sites, relatedness between individuals spanned a wide range, typically from 0.30 to 1.00 (Table 3.2).

Table 3.3: Microsatellite marker diversity estimates for A. gracilipes workers from 20 sites. $\mathrm{N}$, number of individuals genotyped; Alleles, number of alleles detected per locus; $\mathrm{AR}_{10}$, Allelic richness adjusted to the smallest sample size $(\mathrm{n}=10) ; H_{\mathrm{E}}$, expected heterozygosity; $H_{\mathrm{O}}$, observed heterozygosity; $P_{\mathrm{HW}}$, significance values for tests of Hardy Weinberg proportions (heterozygote excess).

\begin{tabular}{cccccccc}
\hline Locus & $\mathbf{N}$ & Size range (base pairs) & Alleles & $\mathbf{A R}_{\mathbf{1 0}}$ & $\boldsymbol{H}_{\mathbf{O}}$ & $\boldsymbol{H}_{\mathbf{E}}$ & $\boldsymbol{P}_{\mathbf{H W}}$ \\
\hline Ano1 & 475 & $97-101$ & 2 & 2.00 & 1.00 & 0.50 & $<0.001$ \\
Ano3 & 475 & $162-178$ & 5 & 2.64 & 1.00 & 0.54 & $<0.001$ \\
Ano4 & 475 & $156-174$ & 2 & 2.00 & 1.00 & 0.50 & $<0.001$ \\
Ano5 & 475 & $116-118$ & 2 & 2.00 & 1.00 & 0.50 & $<0.001$ \\
Ano7 & 475 & $230-248$ & 7 & 3.22 & 0.99 & 0.61 & $<0.001$ \\
Ano8 & 475 & $208-284$ & 17 & 8.29 & 0.93 & 0.87 & 0.083 \\
Ano10 & 475 & $234-242$ & 2 & 2.00 & 1.00 & 0.50 & $<0.001$ \\
\hline
\end{tabular}




\subsubsection{Behavioural differentiation in relation to population genetic structure}

Overall aggression scores were relatively low (generally less than the aggression threshold of 2.00), although aggressive behaviours were observed. These aggressive behaviours typically included gaping of mandibles, twitching, and in one case, one ant subduing and carrying another. The mean aggression scores between ants from the same site did not exceed the aggression threshold, and ranged from 0.70 to 1.23 (Table 3.1; Fig. 3.4). The aggression scores in trials between ants from different sites ranged from 0.83 to 2.27, slightly exceeding the aggression threshold in three instances (Fig. 3.4). Aggression scores were significantly lower within sites than between sites (Fig. 3.4; Wilcoxon rank sum test, $Z=-3.80, P<0.001)$. Mean aggression scores were also positively correlated with genetic and geographic distance.

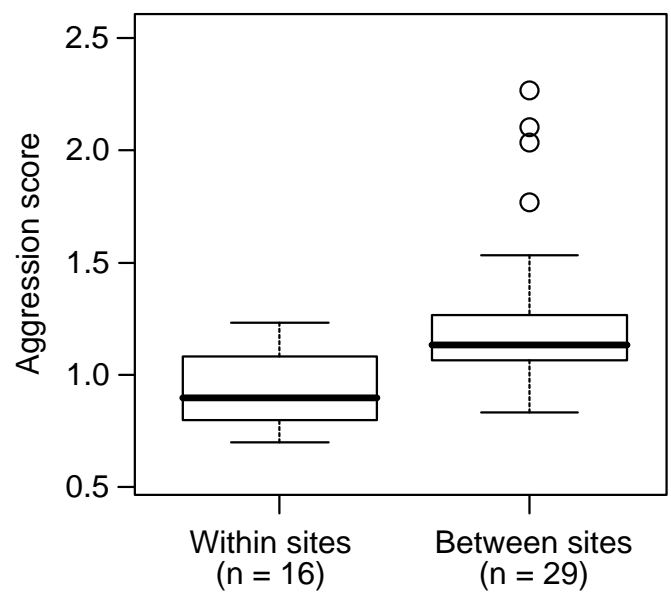

Fig. 3.4: Boxplot comparing aggression scores of A. gracilipes workers within and between sites. Aggression scores were significantly higher between than within sites (Wilcoxon rank sum test, $Z=-3.80, P<0.001)$. The rectangle shows the interquartile range $\left(25^{\text {th }}-75^{\text {th }}\right.$ percentile), the bold line is the median, the whiskers indicate the most extreme data points within 1.5 times the interquartile range from the median, and the open circles represent outliers (points greater than 1.5 times the interquartile range). 


\section{Chapter 3}

Bonferroni corrected Spearman tests were significant for Nei's Distance (Fig. 3.5(a); $r_{s}$ $=0.55, S=6896.92, P<0.001$ ), and geographic distance (Fig. 3.5(b); $r_{s}=0.57, S=$ 0.6467.50, $P<0.001)$. The most genetically or geographically distant sites were not the most behaviourally differentiated (Fig. 3.5). Although a few high scoring points drove the differences in aggression scores, the lowest scores were from within site comparisons.

\subsubsection{Abundance in relation to population genetic structure}

We observed considerable variation in abundance within and among sites (Table 3.2). The mean number of ants observed on card count transects ranged from zero at site M2 (i.e., ant numbers were too low for detection using card counts) to 42.3 at site A1 (Table 3.2). The co-efficient of variation of card counts within sites was 2.5 (range: 0 - 5.7, Table 3.2), and among all sites was 3.6.
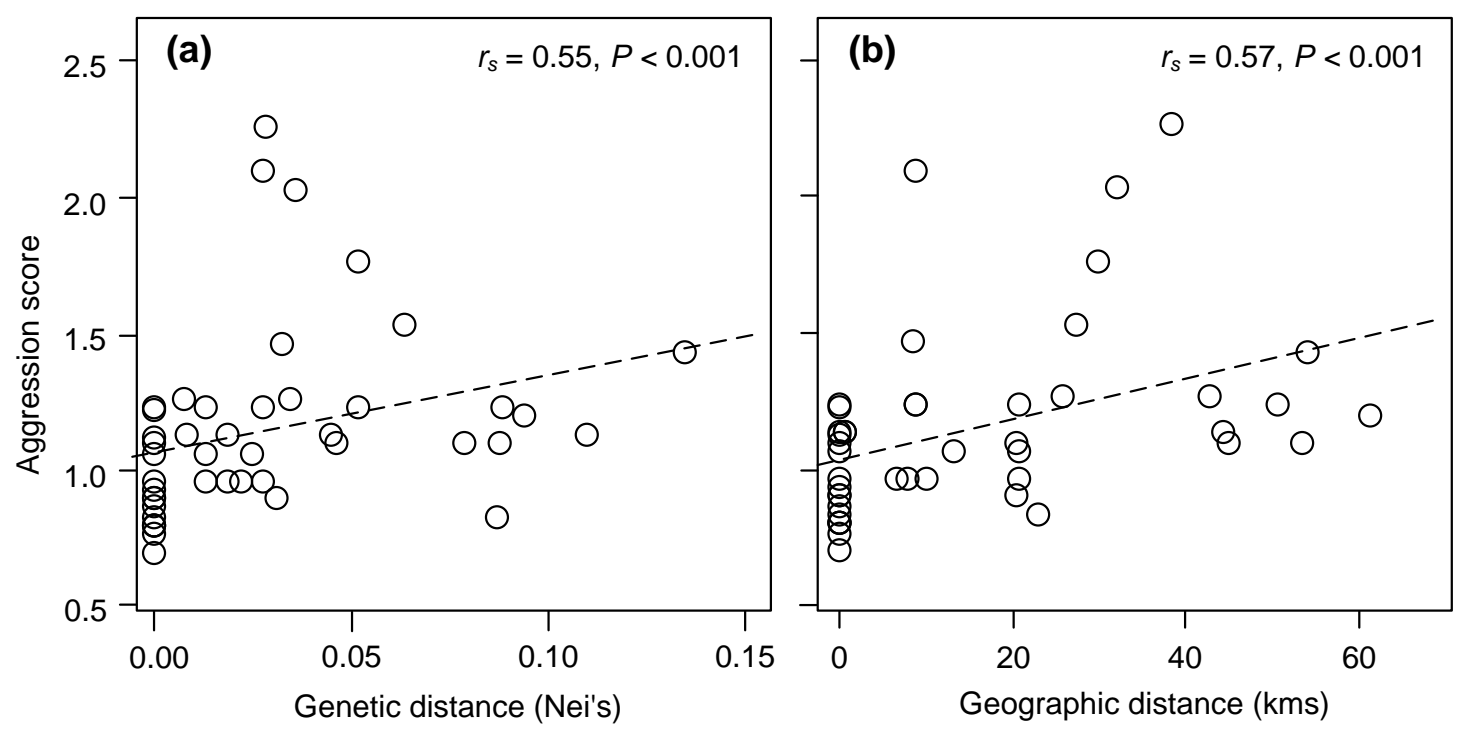

Fig. 3.5: Relationships between aggression score and Nei's distance (a) and geographical distance (b) among nests of A. gracilipes. Spearman's correlation co-efficients $\left(r_{s}\right)$ and significance values $(P)$ of tests are shown for each plot. The dashed lines indicate the line of best fit under a linear model. Aggression scores higher than 2.0 were considered aggressive. 
STRUCTURE, PCAGEN and FLOCK analyses indicated no strong genetic differentiation between sites of lower and higher abundance. The two genetic clusters identified by STRUCTURE were not associated with differences in abundance (Fig. 3.2(a)), and we found no relationship between cluster assignment and abundance (Spearman's $r_{s}=-0.34, S=490.17, P=0.246$ ). We also found no relationship between the variation in abundance within sites $(\mathrm{CV})$ and genetic differentiation (Spearman's $r_{s}=-$ $0.55, S=162, P=0.079)$.

\subsection{Discussion}

\subsubsection{The relationship between population structure and abundance}

The invasion of A. gracilipes in Arnhem Land appears to consist of a single supercolony. We found no association between differences in abundance and population genetic structure. Relatedness within and between sites was high, which is also indicative of a single supercolony, as high relatedness is only observed within supercolonies of $A$. gracilipes (Drescher et al. 2007; Thomas et al. 2010). Relatedness within sites was similar to within colony relatedness found in other A. gracilipes studies (0.37 - 1.00, Drescher et al. 2007; 0.55 - 1.00, Thomas et al. 2010). In contrast, relatedness between sites was higher than other studies, in which between colony relatedness was effectively zero (Drescher et al. 2007; Thomas et al. 2010). Moreover, analysis of mitochondrial DNA shows that A. gracilipes in Arnhem Land share the same haplotype, which provides further corroboration that this is genetically a single population, as individual supercolonies of this species share a single or few haplotypes (Drescher et al. 2007; Thomas et al. 2010). The population genetic differentiation we observed was not correlated with the variation in abundance that we observed within or between sites. The factors responsible for variation in abundance in the Arnhem Land population of $A$. gracilipes remain unclear, but the variation is clearly not due to invasion by ants from different source populations. 


\subsubsection{Divergence of Anoplolepis gracilipes in Arnhem Land}

Although we found that A. gracilipes in Arnhem Land has the genetic and behavioural characteristics of a single supercolony, and thus likely stemmed from a single source, the population is heterogeneous. In most tests we found higher genetic differentiation between distant sites, and higher aggression scores between sites than within sites. In contrast, many supercolonies of other invasive ants such as the L. humile show little or no intra-colonial genetic or behavioural differentiation (Giraud et al. 2002; Tsutsui et al. 2003b; Corin et al. 2007; Suhr et al. 2009), even in supercolonies that span multiple continents (van Wilgenburg et al. 2010). Similarly, workers of the big headed ant, Pheidole megacephala display little genetic or behavioural differentiation, and an absence of isolation by distance across much of the Australian continent (Fournier et al. 2009). A single apparent exception to this homogeneity is that of the Corsican colony of L. humile, which does appear to have differentiated from the main European colony (Blight et al. 2010).

Our results support the scenario proposed by Drescher et al. (2010) to explain divergence within A. gracilipes supercolonies. This scenario entails fragmentation of the colony followed by spatial segregation, which results in population differentiation over time. The mechanism assumes that intranidal mating prevents the homogenising effects of gene flow. Although the mode of reproduction of A. gracilipes is unconfirmed, relatively high within nest relatedness in the species suggests that mating is intranidal (Drescher et al. 2010). Flighted queens have not been observed in Arnhem Land (B. Hoffmann, personal communication), and as relatedness within sites is relatively high, it is likely that mating is intranidal in Arnhem Land. The population of A. gracilipes in Arnhem Land appears to be in the very early stages of divergence. Given sufficient time and spatial segregation we expect the differentiation of A. gracilipes in Arnhem Land to eventually resemble A. gracilipes in Borneo where divergence between populations has resulted in complete behavioural and genetic isolation (Drescher et al. 2010). 
Recent studies suggest that the abandonment of aggression is a plastic trait (Foitzik et al. 2007; Steiner et al. 2007; Cremer et al. 2008; Helanterä et al. 2009; Martin et al. 2011). Although we do not know the behavioural characteristics of the source population, aggression scores above 1.00 have not been observed between ants from the same supercolony in A. gracilipes (Abbott 2005; Abbott et al. 2007), nor has within colony aggression been detected using other methods (Drescher et al. 2007; Drescher et al. 2010). Thus, the three instances of mild aggression we recorded suggest a partial recovery of nestmate discrimination in A. gracilipes in Arnhem Land. The results of Drescher et al. (2010) together with the genetic and behavioural divergence we observed suggests unicoloniality may be a plastic trait in this species.

\subsubsection{Nestmate recognition and aggression}

We have assumed that the variation in behaviour that we found is a result of genetic differentiation, however a number of different factors can affect behaviour among ants. Nestmate recognition and aggressive behaviour are dependent on cuticular hydrocarbon profiles that define recognition cues (Vander Meer and Morel 1998). Nestmate recognition is affected by cuticular hydrocarbon similarity (Suarez et al. 2002), which in turn can be affected by diet (Silverman and Liang 2001; Corin et al. 2007). Although we cannot entirely discount the effect of diet or other environmental aspects, we selected sites based on ecological similarity to minimise the effects of the environment on our results. In addition, genetic, chemical and behavioural differentiation are correlated in geographically diverse A. gracilipes populations in Borneo (Drescher et al. 2010). The correlations found by Drescher et al. (2010) indicate recognition in A. gracilipes has a genetic association. We do acknowledge, however, that environmental cues may have also contributed to the results of our behavioural assays.

A loss of the cues needed for nestmate recognition has been cited as causing the lack of aggression among invasive Argentine ants, and a potential mechanism for the evolution of unicoloniality in the species (Holway et al. 1998; Tsutsui et al. 2000; Giraud et al. 2002). Recently, it has also been suggested that abandoning aggression while retaining nestmate discrimination may be an early stage in the establishment of unicolonial populations, as it enables tolerance of worker exchange among nests (Steiner 


\section{Chapter 3}

et al. 2007), which is a cornerstone of unicoloniality (Helanterä et al. 2009). Although aggression scores in our study typically did not exceed the aggression threshold, scores were clearly higher between ants from different sites, indicating that A. gracilipes in Arnhem Land may discriminate non-nestmates from nestmates. A tolerance of worker exchange within the supercolony is implied by our finding that relatedness within sites was occasionally lower than between sites. Our genetic evidence for tolerance of worker exchange is also consistent with the worker interchange that is observed between $A$. gracilipes nests in the field (B. Hoffmann, unpublished data; Abbott 2005).

\subsection{Conclusion}

Our population structure and behavioural analyses suggest that the numerous and spatially discrete occurrences of $A$. gracilipes ants in Arnhem Land are genetically and behaviourally a single supercolony, and likely stemmed from a single introduction. The variation in abundance in this population is not associated with population genetic differentiation. Unlike the majority of supercolonies of other invasive ants, the $A$. gracilipes supercolony in Arnhem Land is neither genetically nor behaviourally homogeneous. The incipient population differentiation in Arnhem Land, together with the complete differentiation found between supercolonies of the ant in Borneo (Drescher et al. 2010) indicates that colony divergence in this species may be a common occurrence. We speculate that such divergence within ant species may be more common than has been observed to date, as the timescale of divergence may differ greatly among and within species. 


\section{Chapter 4. Higher genetic diversity is associated with fine- scale momentary abundance of an invasive ant}

\subsection{Abstract}

Many introduced species become invasive despite genetic bottlenecks that reduce the genetic diversity of the population, and thus decrease the chances of persistence. By contrast, population genetic bottlenecks have been hypothesised to facilitate the ecological success of unicolonial ants by increasing the genetic similarity between descendent populations, and promoting co-operation between unrelated ants. We investigated these contrasting hypotheses in the yellow crazy ant Anoplolepis gracilipes, which has invaded Arnhem Land in Australia's Northern Territory, and exhibits variation in abundance in the region. We used abundance as a surrogate measure of short-term invasion success, and investigated the relationship between A. gracilipes genetic diversity and variation in abundance, and the density-dependent effects of the ant on native ant species diversity and community structure (i.e., ecological success) at a fine-scale. We also investigated whether selected habitat characteristics contributed to differences in $A$. gracilipes abundance. Our results revealed a significant positive association between measures of $A$. gracilipes genetic diversity and abundance. Ant communities invaded by A. gracilipes were less diverse and differed in structure from uninvaded communities, and these effects were positively density-dependent. Although higher genetic diversity may benefit individual nest clusters, the underlying mechanisms, the direction of the relationship between abundance and genetic diversity, and the implications for longer term invasion success on the wider population are unclear. Although the effects of $A$. gracilipes on the invaded ant community in Arnhem Land appear to be densitydependent, the variability in A. gracilipes abundance may be unlikely to have significant effects on regional native ant diversity over longer timescales. The relative importance of genetic diversity to A. gracilipes abundance may be context-dependent, and genetic diversity may be more important in a specific environment if novel ecological characteristics have less benefit. 


\subsection{Introduction}

The success of invasive species is an apparent genetic paradox, because the loss of genetic diversity that invading populations typically experience should, in theory, limit the chances of invasion success (Allendorf and Lundquist 2003). The genetic bottlenecks that are typically experienced by small founding populations often result in reduced genetic variation relative to the parent population (Sakai et al. 2001). In the short term small introduced populations are thus susceptible to inbreeding and strong genetic drift, which may further erode genetic variation. In the longer term a lack of genetic variation may impede the potential for adaptive evolution. This apparent genetic paradox may be resolved if invading populations have higher genetic diversity than source populations owing to high propagule pressure (Kolbe et al. 2004; Roman and Darling 2007), the genetic diversity measured by commonly used molecular markers such as microsatellites does not reflect the total diversity in the genome (Väli et al. 2008), or if genetic variation is unimportant, as is observed in clonally reproducing species (Baker 1995; Dybdahl and Drown 2011).

In some cases colonisation success may be enhanced by a reduction in genetic diversity. Greater genetic similarity in the introduced population owing to a small number of founders could result in increased co-operation, thus enhancing ecological success (Suarez et al. 1999; Tsutsui et al. 2000). It has been suggested that population bottlenecks during the introduction event have promoted large-scale unicoloniality, and thus the invasion success, of the well-studied Argentine ant Linepithema humile. This is owing to a breakdown in kin recognition, so that individuals that are not related cooperate because they perceive each other as similar (Tsutsui et al. 2000). Although this bottleneck hypothesis has been disputed by others (Vogel et al. 2010), genetic bottlenecks may also aid in invasion success through the purging of deleterious alleles (Schmid-Hempel et al. 2007). As the bottleneck hypothesis specifically refers to differences between the native and introduced ranges (Suarez et al. 1999; Tsutsui et al. 2000), the putative benefits of a loss of genetic diversity may or may not persist subsequent to the initial bottleneck. 
Invasion success can be difficult to define and measure. However, the effects of invasive ant species on the recipient community are typically density-dependent (e.g., Ross et al. 1996; O'Dowd et al. 2003; Le Breton et al. 2005; Krushelnycky and Gillespie 2008; Lester et al. 2009). The high abundance attained by invasive ants strengthens their competitive ability relative to native ants and furthers their ecological dominance through numerical monopolisation of resources (e.g., Holway 1999; Human and Gordon 1999; Morrison 2000; Rowles and O'Dowd 2007; Sagata and Lester 2009). In most organisms the census population size $(N c)$ and effective population size $(N e)$ are coupled, and genetic diversity typically increases with increasing population size. In ant species, however, worker abundance does not necessarily reflect effective population size, as reproduction involves relatively few individuals, and these are not workers (Wilson 1963). However, worker genotypic diversity is representative of the genetic diversity of the colony, as it reflects the contribution of reproductive individuals. As effective population size and worker abundance are independent in ants, worker abundance, along with the alteration of community structure in the invaded community (e.g., Sanders et al. 2003) are proxies by which short-term, or momentary, invasion success can be assessed. Here we define momentary invasion success as the ability for a species to reach sufficient abundance to cause a reduction in species diversity and a change in the structure of the community to which they have been introduced.

Anoplolepis gracilipes is one of the most widespread and damaging invasive ants (Holway et al. 2002). It can occur at very high densities (> 2000 ants $\mathrm{m}^{2}$ ) and can cause substantial ecosystem change (O'Dowd et al. 1999; O'Dowd et al. 2003; Abbott 2006b). In Arnhem Land in the Northern Territory of Australia, the abundance of this species exhibits considerable spatial variation. Unlike A. gracilipes invasions in Samoa (Savage et al. 2009) and on Christmas Island (O'Dowd et al. 2003), there appear to be no obvious single ecological mechanisms promoting differences in abundance, such as mutualisms with Homoptera (Young et al. 2001), nor are there clear associations between the abundance of this ant and anthropogenic disturbance (Hoffmann and Saul 2010). We therefore hypothesised that there could be an association between genetic diversity and the variation in abundance of A. gracilipes in Arnhem Land. 


\section{Chapter 4}

Although the genetic structure and behaviour of A. gracilipes in Arnhem Land is consistent with a single population, population genetic structure is not correlated with variation in abundance (Chapter 3). The population is somewhat heterogeneous and hypothesised to be in the process of divergence (Chapter 3). Thus, the variable but spatially discrete occurrences of A. gracilipes in Arnhem land resemble a mosaic of distinct nest clusters or 'meta-colony' (sensu Heller et al. 2008). In this study we use 'population' to refer to the entire distribution of A. gracilipes in Arnhem Land, and 'clusters' (or plots) to refer to individual localised occurrences of the ant.
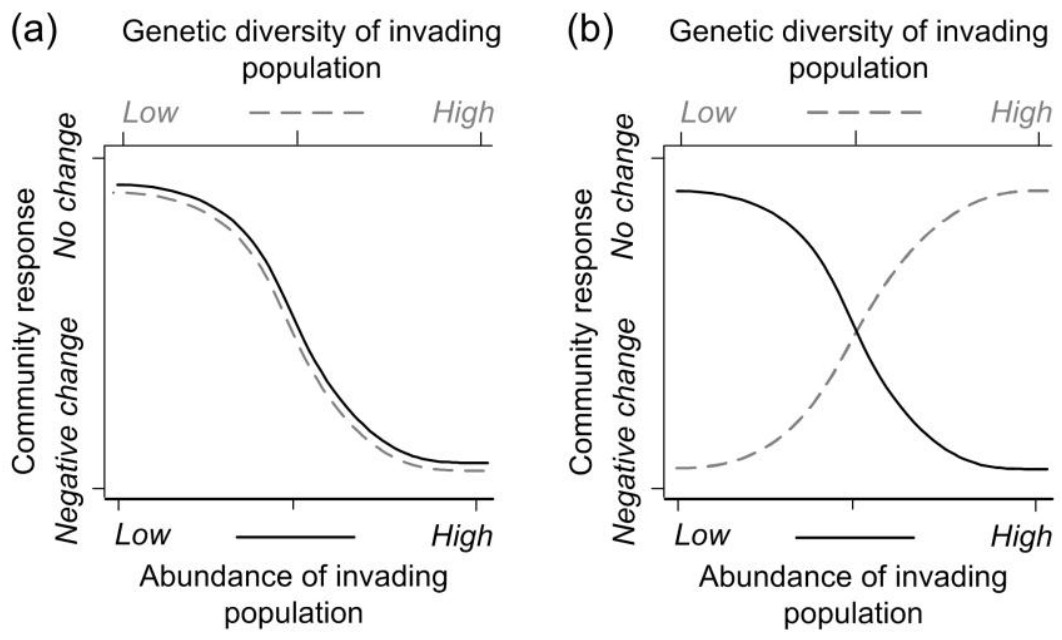

Fig. 4.1: Our hypothesised relationships between the genetic diversity (e.g., allelic richness or genotypic richness) and abundance of the invading population, and relative influence on invaded communities under scenarios of: (a) a positive relationship between genetic diversity and abundance; and (b) a negative relationship between genetic diversity and abundance.

The aim of the present study is to explore the relationships between genetic diversity, abundance and ecological success. Chapter 3 investigated population genetic differentiation (i.e., beta diversity), while in this study we extend the analysis to investigate genetic diversity at the local scale at which ants are likely to interact (i.e., alpha diversity). If higher genetic diversity facilitates momentary invasion success, higher genetic diversity and greater A. gracilipes abundance would be co-observed, and would be associated with negative effects on the invaded community (Fig. 4.1(a)). Conversely, if reduced genetic diversity facilitates momentary success, lower genetic diversity would be 
correlated with greater A. gracilipes abundance, and with negative effects on the invaded community (Fig. 4.1(b)). To test these competing hypotheses we address three specific questions. First, is there an association between A. gracilipes genetic diversity and abundance? Second, are there differences in native ant species diversity and community structure between invaded and uninvaded communities? Third, is there an association between $A$. gracilipes abundance and native ant species diversity in the invaded community? Further, to evaluate an effect of environment we investigate if habitat characteristics are associated with variation in A. gracilipes abundance.

\subsection{Methods}

\subsubsection{Study area}

Arnhem Land is located in the monsoonal tropics of Australia's Northern Territory (Fig. 4.2). The region experiences daytime high temperatures ranging between 17 and $33^{\circ} \mathrm{C}$, and seasonal rainfall (December to July) of approximately $1200 \mathrm{~mm}$. The vegetation is primarily fire-prone savanna woodland. Although it is not known when A. gracilipes arrived in the region, it was first detected in 1982 (Majer 1984). In 2009 the ant was patchily distributed throughout $16,000 \mathrm{~km}^{2}$ in Arnhem Land (Fig. 4.2), mainly in anthropogenically undisturbed and ecologically intact sites. Genetic and behavioural analyses of population structure suggest this population stemmed from a single source (Chapter 3). However, the genetic structure and intra-specific behaviour of the population is heterogeneous, which suggests the population is in the process of divergence (Chapter 3). In this study we extend the analysis of Chapter 3 to investigate genetic diversity at the scale at which ants are likely to interact.

\subsubsection{Ecological surveys}

Nine study sites were selected for an ecological survey undertaken in July 2009 (Fig. 4.2), which intersect with the sites sampled in Chapter 3. The differences between these sampling sites and those of Chapter 3 are the additions of plots D1, M1, K6, K7, and K2. We selected sites with similar habitat characteristics in a stratified random fashion. All sites had a dominant canopy of Eucalyptus tetrodonta, an understorey primarily 


\section{Chapter 4}

consisting of Acacia spp., grasses and leaf litter, and similar drainage and topography. At each of the nine sites we selected two visually similar plots in areas invaded by $A$. gracilipes. Plots were spaced at least $100 \mathrm{~m}$ apart to ensure their independence. The largest A. gracilipes foraging distance recorded in this region is $35 \mathrm{~m}$, and within this distance ants freely move between nests (B. Hoffmann unpublished data). The presence of at least one A. gracilipes nest was confirmed in invaded plots, and we paired these invaded plots with nearby plots where the ant was absent. For the purposes of our study we inferred that A. gracilipes workers sampled in plots were representative of nest clusters within foraging range of the plot.

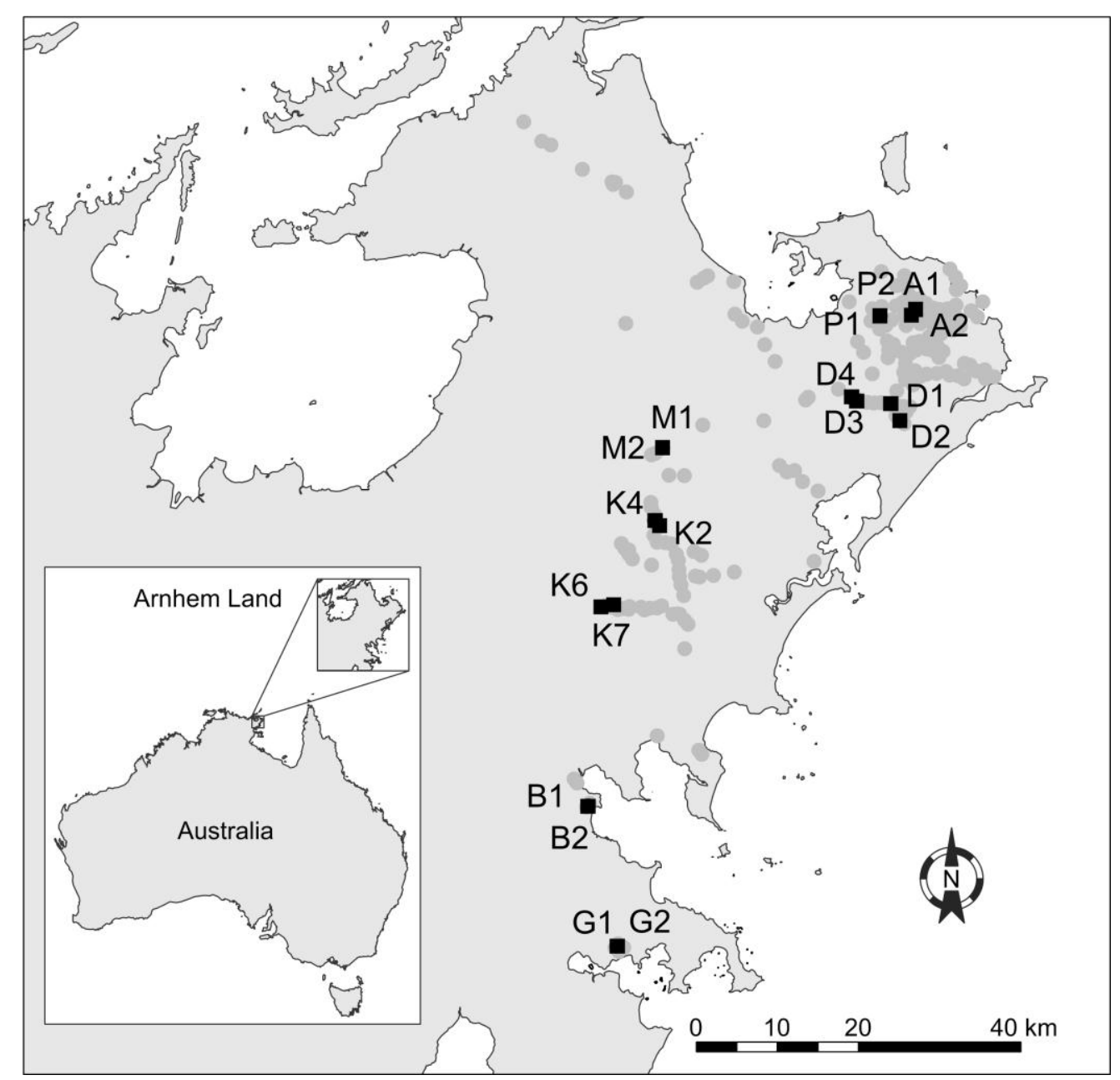

Fig. 4.2: Anoplolepis gracilipes distribution in Arnhem Land in the Northern Territory, Australia at the time of sampling in July 2009 (grey circles), and sampling plots (black squares with site codes). 
At each plot we installed 16 pitfall traps (45 $\mathrm{mm}$ diameter) haphazardly placed approximately $1 \mathrm{~m}$ apart in a $4 \times 4 \mathrm{~m}$ grid around an A. gracilipes nest. Traps were $2 / 3$ filled with propylene glycol and left for 48 hours. All ant species collected were counted and identified to species level. Species that could not be named were assigned to species groups according to Andersen (2000). Voucher specimens for all species were retained at the Tropical Ecosystems Research Centre in Darwin or Victoria University of Wellington.

In addition to A. gracilipes counts in pitfall traps, to assess population densities we also measured the abundance of $A$. gracilipes at the plot level based on forager activity, as described in Chapter 3. Briefly, this method uses a count of the number of ants crossing a laminated card in a $30 \mathrm{~s}$ period (Green et al. 2004). At each site we measured $A$. gracilipes activity at 11 stations spaced at $5 \mathrm{~m}$ intervals along three replicate $50 \mathrm{~m}$ transects spaced $10 \mathrm{~m}$ apart. Counts at all stations within a transect were summed, and the mean value of the three replicate transects was used as an index of relative abundance between plots. Our card counts ranged from 0 to 42 , and were highly correlated with pitfall trap counts for each plot (Spearman's rank correlation $R_{s}=0.80, S=195, P<$ 0.001, conducted in R v 2.13.1 (Ihaka and Gentleman 1996; R Development Core Team 2011)). We used the pitfall trap data in all analyses as these data were collected at the same spatial scale as the genetic diversity and habitat data.

Native ant species were also assigned to the functional groups used in studies of Australian ant communities (Andersen 1995): Dominant Dolichoderinae (DD); Subordinate Camponotini (SC); Climate Specialists [sub-divided into Hot (HCS) and Tropical (TCS)]; Generalised Myrmicinae (GM); Opportunists (OPP); Cryptic Species (CS); and Specialist Predators (SP). In addition to A. gracilipes we found three other nonnative ant species: Monomorium floricola (one ant in one uninvaded plot), Tetramorium simillimum (62 ants in one invaded and five uninvaded plots) and Paratrechina longicornis (14 ants in two uninvaded plots). As these species are not known to have any effects on savanna ant communities (Hoffmann and Saul 2010), we included their data with the native species. 


\section{Chapter 4}

We recorded a number of habitat attributes at the plot level that we considered could contribute to differences in local ant community structure and abundances of $A$. gracilipes: availability of potential A. gracilipes nest sites (the proportion of the plot occupied by logs and tree basal area, as A. gracilipes often nest at the base of trees); canopy cover (measured with a spherical densiometer); the abundance of Acacia spp. (a count of the number of plants in the plot); and leaf litter depth (a mean of four estimates in the plot). Availability of nest sites has been considered to have a major influence on $A$. gracilipes population size (Haines and Haines 1978a). Denser canopy cover can facilitate extended foraging time for ants in extreme heat. Acacia spp. offer a potential novel carbohydrate resource, which can enhance ant abundance (Davidson 1997 and references therein). Novel carbohydrate resources are an important variable as they facilitate invasion of A. gracilipes on Christmas Island (O'Dowd et al. 2003) and Samoa (Savage et al. 2009). Finally, leaf litter depth is an approximate indicator of time since fire, with deeper leaf litter occurring in unburnt areas (Cook 2003; Russell-Smith et al. 2009). Fire is a major driver of vegetation structure and composition in savannas, which in turn are major drivers of ant community composition (Andersen and Hoffmann 2011). These habitat attributes are henceforth referred to as nest sites, canopy, Acacia and litter respectively.

\subsubsection{Molecular analyses}

For molecular analyses, we haphazardly selected A. gracilipes workers from all nests in our study plots in July 2009 (Fig. 4.2). We supplemented these with additional ants collected from pitfall traps at the same site when allele discovery curves did not flatten (Supplementary Fig. 4.1). Ants were stored in $95 \%$ ethanol at $4{ }^{\circ} \mathrm{C}$. We extracted genomic DNA using a modified Chelex protocol (Sepp et al. 1994). Individual workers were placed in microcentrifuge tubes, ground with sterile plastic pestles, and $150 \mu 1$ of a $10 \%$ w/v Chelex-100 resin solution was added. The tubes were centrifuged briefly, boiled for 15 minutes, chilled on ice for five minutes, and centrifuged at $15,000 \times g$ for 15 minutes at $4^{\circ} \mathrm{C}$. The supernatant containing DNA was stored at $4^{\circ} \mathrm{C}$. 
Microsatellite molecular markers were used to assess the genetic diversity of $A$. gracilipes at the 18 sampled plots. Workers were genotyped for seven microsatellite loci: Ano1, Ano3, Ano4, Ano5, Ano7, Ano8 and Anol0 (Feldhaar et al. 2006), using the methods outlined in Chapter 3. Fluorescent dyes used were FAM (Ano4, Ano5 and Ano8) and VIC (Anol, Ano3, Ano7 and Ano10). Polymerase Chain Reactions were performed using the thermal cycling conditions specified by Feldhaar et al. (2006), with modification for M13 primers (Schuelke 2000). Amplified products were analyzed using the LIZ size standard on a 3730 Genetic Analyzer, and visualised and scored using Genemapper v 3.7 (both Applied Biosystems).

In order to determine whether sufficient worker ants were genotyped to represent allelic diversity, we generated allele discovery curves for the most polymorphic locus (Ano8) using the 'PopGenKit' package (Rioux Paquette 2011) in R, with jack-knifing using 1000 replicates. A flattening of allele discovery curves with increasing sample size indicates that the samples genotyped are a fair representation of the allelic diversity in the population. Where possible, we genotyped additional ants where discovery curves did not flatten (Supplementary Fig. 4.1), until there were no further ant samples remaining.

The majority of the 539 A. gracilipes workers genotyped were heterozygous at all loci, as is typical for this species (Drescher et al. 2007; Drescher et al. 2010; Thomas et al. 2010; Chapters 2 and 3). Forty ants were homozygous at the Ano8 locus, and one was homozygous at the Ano5 locus. The undetermined reproductive mode of A. gracilipes (Drescher et al. 2007; Chapter 2), and the existence of populations of A. gracilipes workers heterozygous at all loci (Thomas et al. 2010) indicates that homozygous loci could reflect allele dropout and thus possibly be biologically inaccurate. Therefore we conducted analyses with and without these worker genotypes as their presence may have inflated genotypic diversity estimates. Although we found no major differences between the two datasets we have chosen to report the analyses excluding these workers. These analyses were based on genotyping of 20 to 35 workers from each of the 18 plots (mean \pm $\mathrm{SE}=27.67 \pm 1.07, \mathrm{n}=498)$. The removal of samples with homozygous genotypes resulted in a smaller sample size for some plots compared with Chapter 3, and the addition of genotypes as a result of allele discovery curves not flattening increased the sample sizes in other plots. 


\section{Chapter 4}

\subsubsection{Statistical analyses}

For all statistical tests a significance level of $\alpha=0.05$ was used. For all analyses, effect sizes were interpreted as small $\left(R^{2} \sim 0.01, R_{S} \sim 0.10\right)$, medium $\left(R^{2} \sim 0.09, R_{S} \sim 0.30\right)$, or large $\left(R^{2} \sim 0.25, R_{s} \sim 0.50\right)$, according to Cohen (1988). We conducted all analyses at the plot level.

\subsubsection{Is there an association between Anoplolepis gracilipes genetic diversity and abundance?}

Rarefied allelic richness was calculated in $\mathrm{R}$ using the 'PopGenKit' package (Rioux Paquette 2011), with jack-knifing of 1000 replicates. We estimated other genetic diversity parameters, including genotypic richness $(\mathrm{G})$ and unbiased genotypic diversity $\left(R_{U}\right)$, Shannon entropy (H'), and Simpson's index of diversity (D) using Genclone v 2.0 (Arnaud-Haond and Belkhir 2007). We then derived Hill's numbers from these measures using the same naming convention as species diversity $\left({ }^{0} \mathrm{D}=\right.$ allelic richness (including only informative loci); ${ }^{1} D=\exp$ [Shannon's H']; and ${ }^{2} D=1$ /Simpson's D). We chose richness-based measures of diversity as these should be sensitive to the elimination of rare alleles by drift (Nei et al. 1975; Allendorf 1986). We chose genotypic diversity measures because the undetermined reproductive mode of A. gracilipes could involve clonality, and genotypic diversity would provide an estimate of the number of clones present. The relationships between diversity measures $\left({ }^{0} D,{ }^{l} D\right.$ and ${ }^{2} D$, and $\left.R_{U}\right)$ and differences in the abundance of A. gracilipes were analysed using Spearman's rank correlation implemented in $\mathrm{R}$. We visualised the relationships between variables by fitting smoothed lines with the loess function in $\mathrm{R}$ using a span of 0.9 . 


\subsubsection{Are there differences in native ant species diversity and community structure between invaded and uninvaded communities?}

Species diversity measures (richness, Shannon's H' and dominance / Simpson's D) were estimated by individual-based rarefaction (Hurlbert 1971) using 1000 permutations implemented in EcoSim v 7 (Gotelli and Entsminger 2007). The lowest number of native ants sampled in a plot (52 individuals) was the rarefied sample size. We derived Hill's numbers from these measures following the terminology of Jost (2006): ${ }^{q} D$ where ${ }^{q}=1,2$ or 3 (i.e., ${ }^{0} D=$ richness; ${ }^{l} D=\exp \left[\right.$ Shannon's H']; and ${ }^{2} D=1 /$ Simpson's D). These measures assign different weights to the relative abundance of species, with the importance of rare species decreasing as $q$ increases, and together provide a biologically meaningful overview of the diversity of the community. To test if these measures of species diversity differed between invaded and uninvaded plots, we used Wilcoxon rank sum tests (with Monte-Carlo resampling to estimate $P$ values), using the 'coin' R package (Hothorn et al. 2008). We used the $Z$ score estimate to convert $Z$ statistics to an $R^{2}$ effect size using the formula $R^{2}=Z^{2} / \mathrm{N}$ (Rosenthal 1991). Species accumulation curves (samplebased rarefaction curves) were generated for invaded and uninvaded sites using EstimateS v 8.2.0 (Colwell 2009). Pitfall traps were used as the sample and we generated curves for each site individually (Supplementary Fig. 4.2).

To assess if native ant community structure differed between invaded and uninvaded plots we used non-metric Multi-Dimensional Scaling (MDS) implemented in Primer v 6.1.11 (Clarke and Gorley 2006). We log-transformed data to even out the effects of rare and abundant species, and used the Bray-Curtis index as a similarity measure as recommended by Clarke and Warwick (2001). The MDS was run over 1000 iterations using Kruskal stress formula 1 and a minimum stress of 0.01 . We tested for significant differences between invaded and uninvaded plots using PERMANOVA+ run over 99999 permutations, and checked for constant dispersion among groups using PERMDISP (Anderson et al. 2008). We used SIMPER analysis to assess how individual species contributed to differences between invaded and uninvaded plots (Clarke and Warwick 2001). 


\section{Chapter 4}

\subsubsection{Is there an association between Anoplolepis gracilipes abundance and native ant species diversity in the invaded community?}

If higher abundance were associated with negative effects on the invaded community, we would have expected a decrease in species diversity as abundance increased. To determine if species diversity measures $\left({ }^{0} D,{ }^{l} D\right.$ and $\left.{ }^{2} D\right)$ were correlated with variation in the abundance of A. gracilipes we used Spearman's rank correlation in R. We visualised the relationships between variables by fitting smoothed lines with the loess function in $\mathrm{R}$ using a span of 0.9.

\subsubsection{Are habitat characteristics associated with variation in Anoplolepis gracilipes abundance?}

We used non-parametric multiple regression with generalised additive models and automatic spline smoothing implemented in the 'mgcv' R package (Wood 2006) to test if the habitat attributes we measured contributed to differences in A. gracilipes abundance. We modelled A. gracilipes abundance as the dependent variable, site as a fixed factor, and litter, canopy, Acacia, nest sites as the independent variables. We ran models using all data, and data for invaded plots alone. As a single model could not be fit with all the model terms included, we used forward model selection, excluded terms that explained the least of the variation in the data, and selected the best models as those that explained most of the variation in the data. We visualised the relationships between environmental variables among plots using principal component analysis (PCA) in Primer v 6.1.11. 


\section{$4.4 \quad$ Results}

\subsubsection{Is there an association between Anoplolepis gracilipes genetic diversity and abundance?}

We found a significant correlation between most measures of genetic diversity and $A$. gracilipes abundance. Anoplolepis gracilipes was the most abundant species in all invaded plots, but no single species was universally the most abundant in uninvaded plots. Anoplolepis gracilipes abundances ranged from 62 to 5288 ants per invaded plot (mean \pm SE: $1134 \pm 328$ ants), and from zero to 1016 ants per pitfall trap (mean \pm SE: 71 \pm 6 ants). Genetic diversity measures were positively correlated with each other, with the exception of ${ }^{2} D$, which was negatively correlated with the other measures (Table 4.1; Fig. 4.3). The abundance of $A$. gracilipes was also positively correlated with all genetic diversity measures with the exception of ${ }^{2} D$, which was negatively correlated with abundance (Fig. 4.3).

Although the positive relationship between A. gracilipes abundance and genetic diversity was not significant for ${ }^{0} D\left(S=674, P=0.109, R_{S}=0.30\right)$, this relationship nonetheless had a medium effect size. These relationships were positive and significant for ${ }^{1} D\left(S=431, P=0.008, R_{S}=0.55\right)$, and unbiased genotypic diversity $(S=540, P=$ $\left.0.034, R_{s}=0.44\right)$, and negative and significant for ${ }^{2} D\left(S=1514, P=0.007, R_{S}=0.56\right)$. All significant relationships also had large effect sizes. The relationships between abundance and genetic diversity were not linear, and instead were best fit by lines that tended toward a sigmoidal or inverse exponential function. 


\section{Chapter 4}
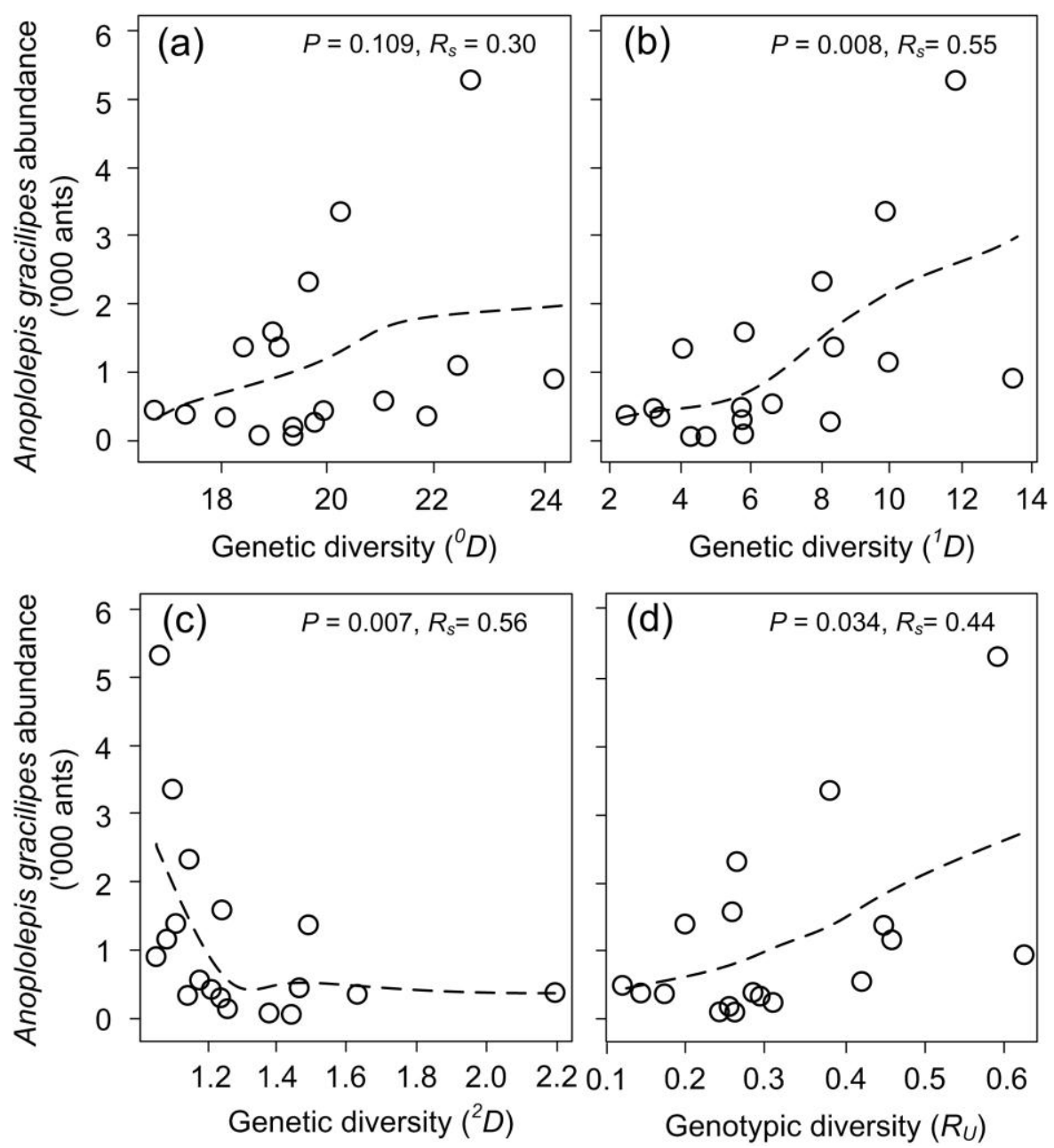

Fig. 4.3: The relationship between A. gracilipes abundance and genetic diversity: (a) ${ }^{0} D$ (allelic richness); (b) ${ }^{1} D$ (exp[Shannon's H']); (c) ${ }^{2} D$ (1/Simpson's index); (d) genotypic diversity. Dashed lines indicate the smoothed spline line of best fit with a span of 0.9.

\subsubsection{Are there differences in species diversity and community structure between invaded and uninvaded communities?}

Seventy species in total were found in our survey (61 in uninvaded and 49 in invaded plots), and the number of species per plot ranged from 7 to 22 (mean \pm SE: $12 \pm 0.6$ species in invaded plots and $15 \pm 0.8$ species in uninvaded plots). Mean native ant abundances ranged from 52 to 918 ants per plot (mean \pm SE: $252 \pm 42.0$ ants in invaded and $319 \pm 56.5$ ants in uninvaded plots). 


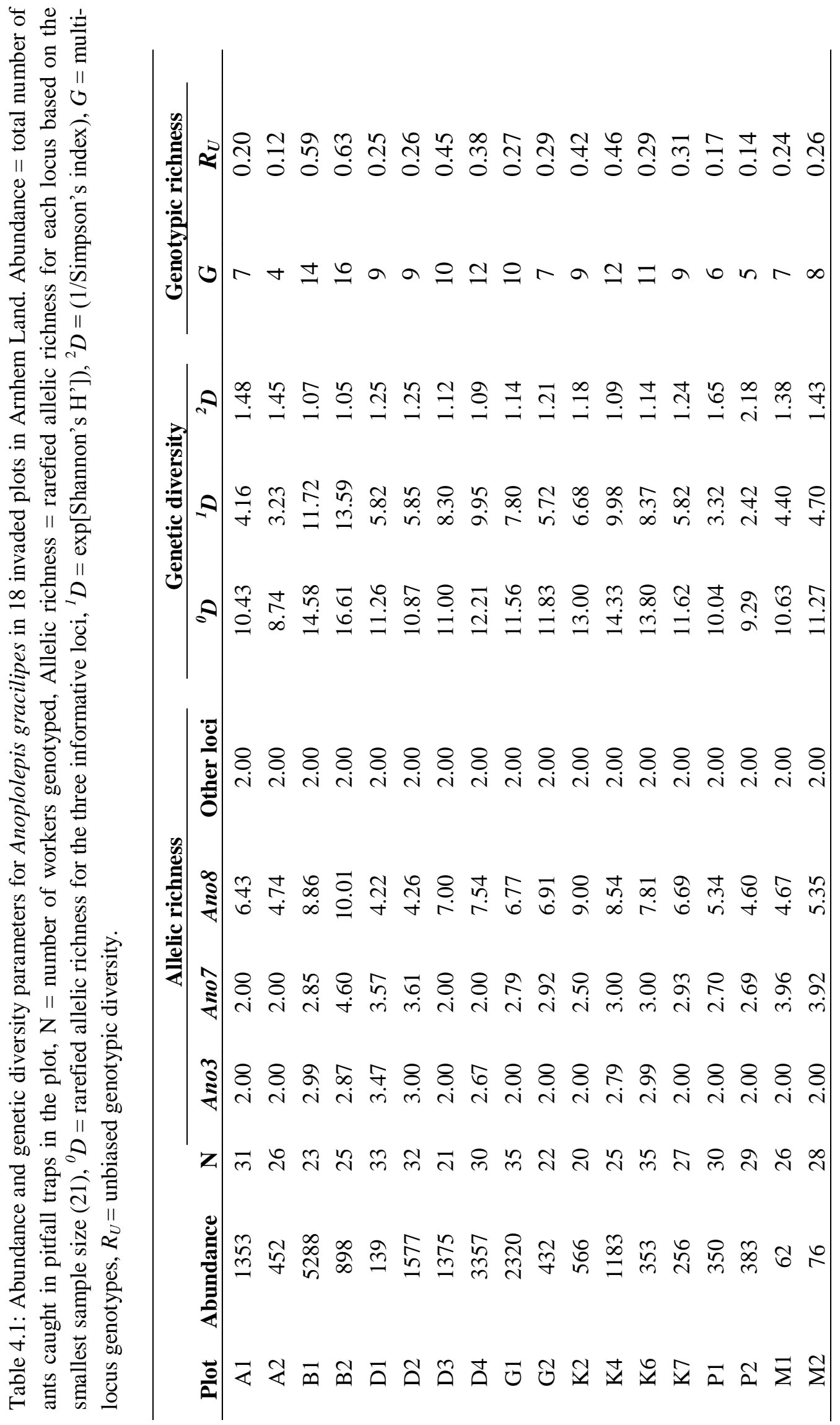




\section{Chapter 4}

Native ant species diversity differed between invaded and uninvaded plots, but only species richness $\left({ }^{0} D\right)$ was significantly higher in uninvaded plots than invaded plots (approximative Wilcoxon Mann-Whitney Rank Sum Test, $Z=-1.96, R^{2}=0.11, P=$ 0.047). Other species diversity measures did not significantly differ between invaded and uninvaded plots, but the observed effect sizes were progressively lower as $q$ increased. $\left({ }^{1} D: Z=-1.76, R^{2}=0.09, P=0.077 ;{ }^{2} D: Z=-1.24, R^{2}=0.04, P=0.227\right)$. As rare species are assigned a lower importance in the ${ }^{1} D$ and ${ }^{2} D$ measures, this decreasing effect size as $q$ increases suggests that rare species are less likely to co-occur with A. gracilipes.

Three-dimensional MDS revealed clear differences in community structure between uninvaded and invaded plots (Fig. 4.4). The results of PERMANOVA+ analysis revealed significant differences between invaded and uninvaded plots (Pseudo $F=4.43_{1,34}$, $P[$ perm $]<0.001)$. PERMDISP analysis found that dispersions were homogeneous among groups $\left(F=2.13_{1,34}, P[\right.$ perm $\left.]=0.165\right)$, so the differences between groups were not owing to differences in dispersion within groups.

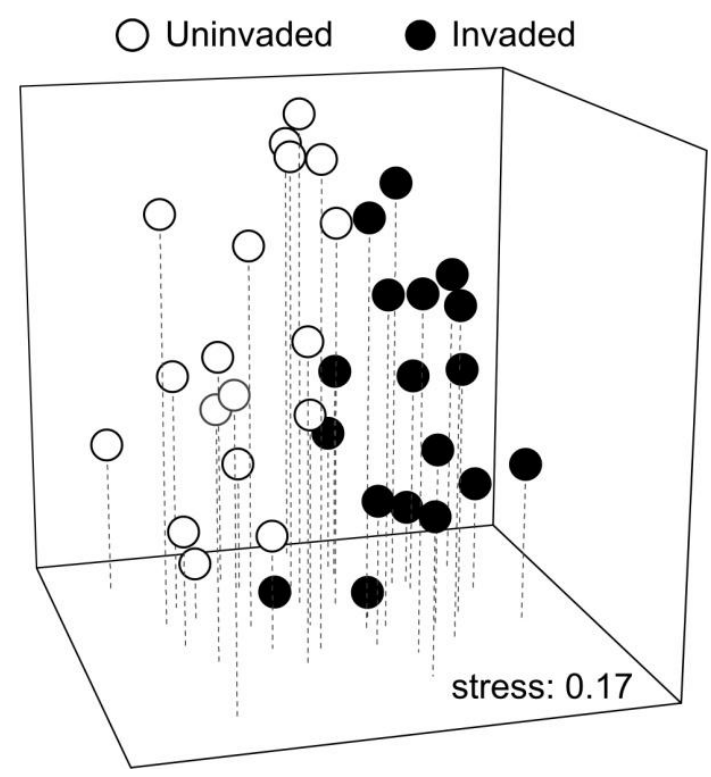

Fig. 4.4: Three-dimensional MDS plot of A. gracilipes invaded and uninvaded plots based on Bray-Curtis similarity of $\log (\mathrm{X}+1)$ transformed abundance data. Anoplolepis gracilipes were excluded from the analysis. The accompanying PERMANOVA+ analysis revealed significant differences between invaded and uninvaded plots (Pseudo $F=4.43_{1,34}, P[$ perm $]<$ 0.001). 
SIMPER analysis found that invaded plots were less likely to include dominant species and more likely to contain insinuating species than uninvaded plots. $90 \%$ of the dissimilarity between invaded and uninvaded plots was attributable to 33 ant species, while $50 \%$ of the dissimilarity was attributable to 11 species. The abundances of a number of these species differed markedly between invaded and uninvaded plots (Table 4.2). The contribution of different functional groups also differed between invaded and uninvaded plots. Invaded plots had fewer Dominant Dolichoderinae (DD) and Tropical Climate Specialists (TCS) and more Generalised Myrmicinae (GM). Ecologically dominant species (DD and Oecophylla smaragdina (TCS)) were absent from many but not all invaded plots. In invaded plots $O$. smaragdina occurred at M1, M2 and P2. Three species of DD occurred in invaded plots. Iridomyrmex pallidus was found in plots B2 and G2, and Iridomyrmex sp. 1 anceps group was found in plots D3, D4, G2, K4, K7 and P2. Iridomyrmex sp. 3 myobergi group occurred at plot M2. When they occurred in invaded plots these species had lower abundance than when they were found in uninvaded plots (Table 4.2). Iridomyrmex reburrus was only found in uninvaded plots. 


\section{Chapter 4}

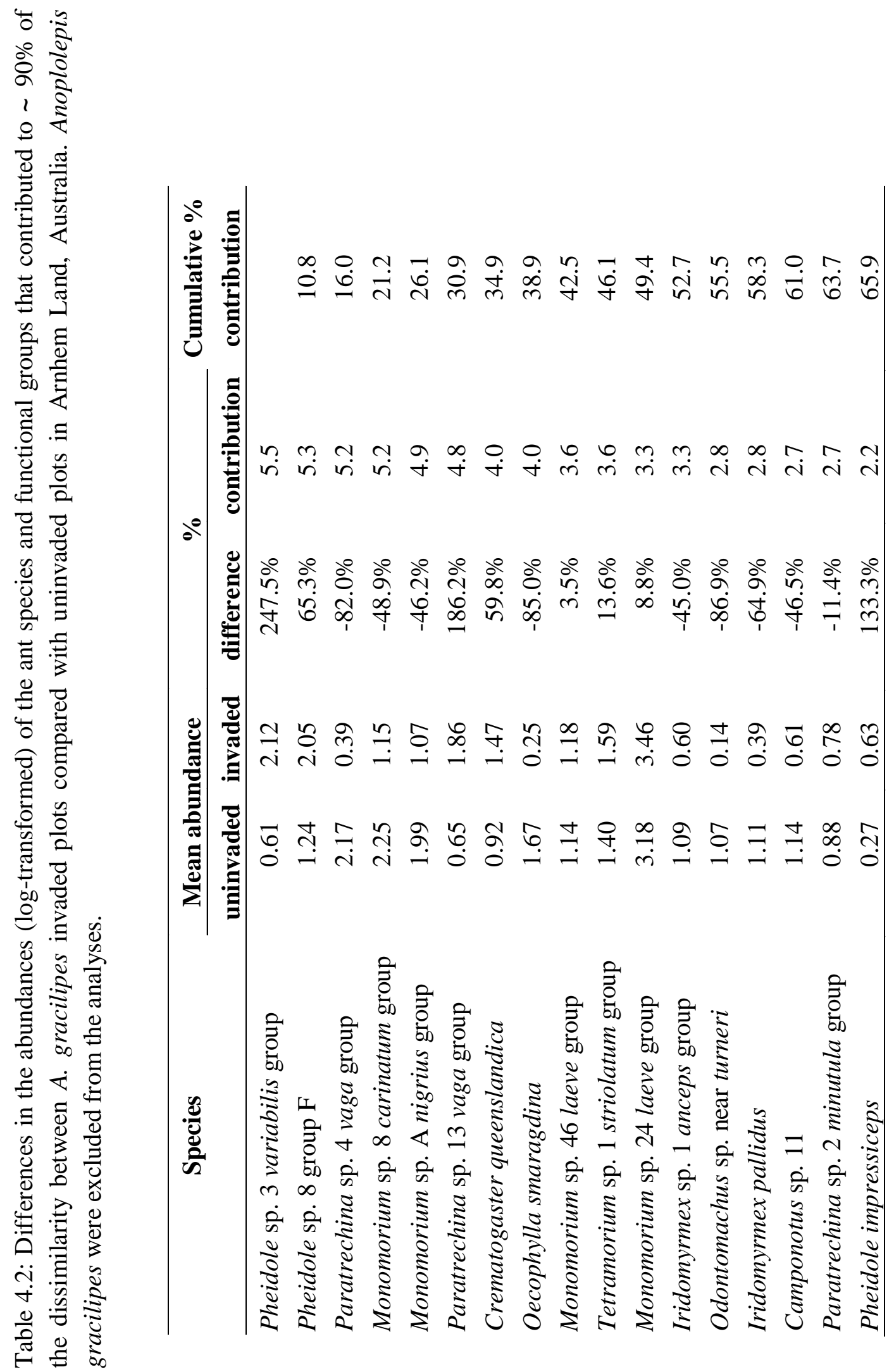




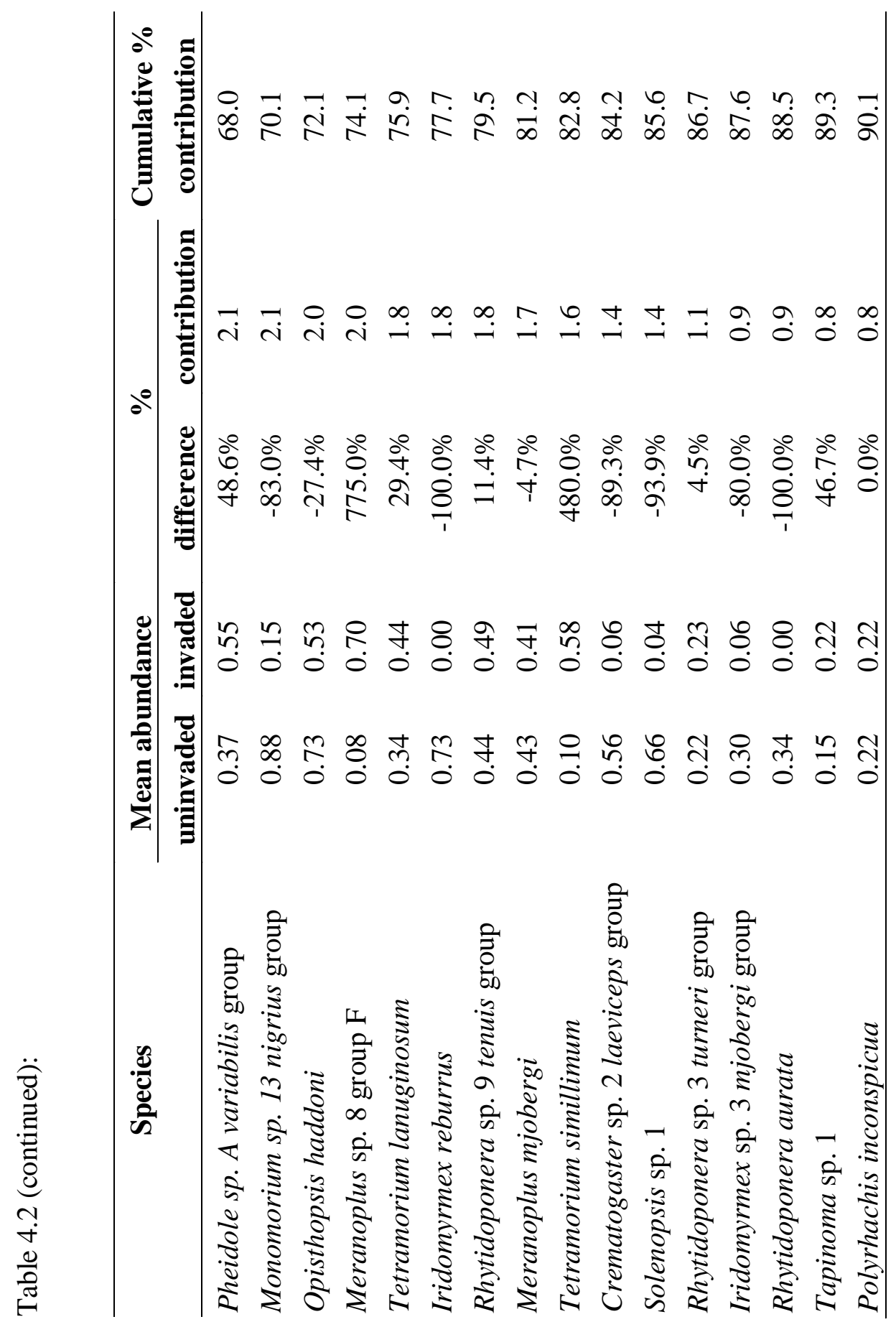




\section{Chapter 4}

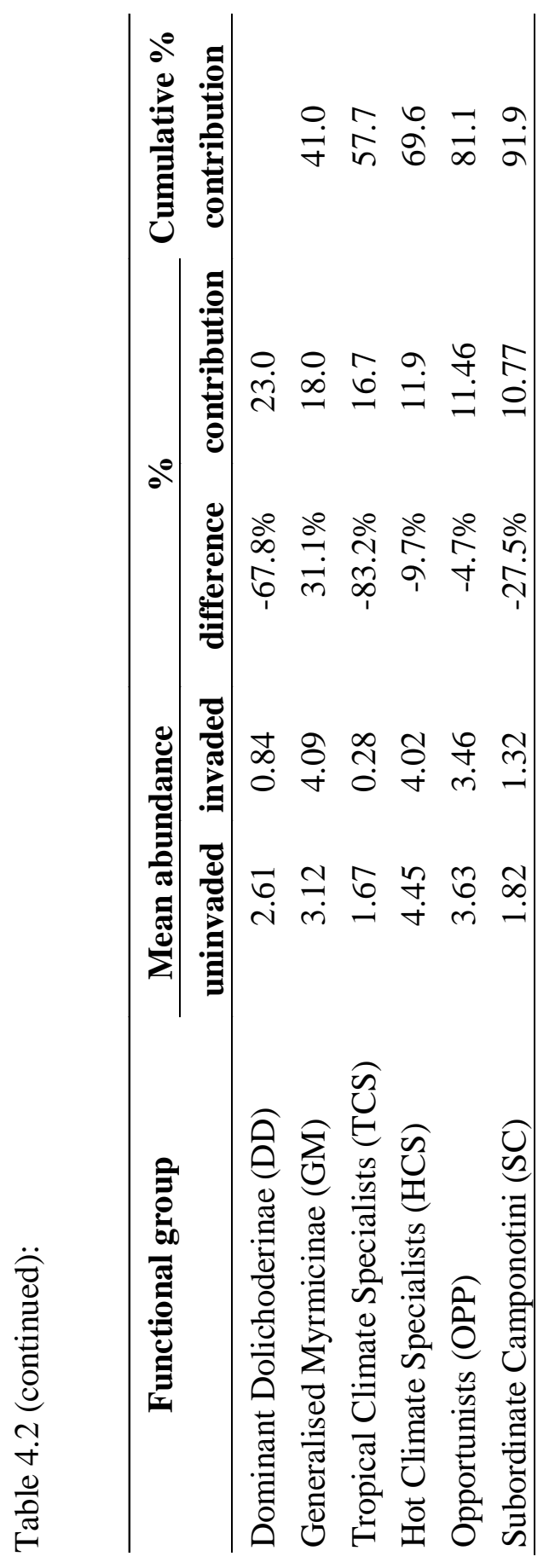


4.4.1.3 Is there an association between Anoplolepis gracilipes abundance and species diversity of the invaded community?
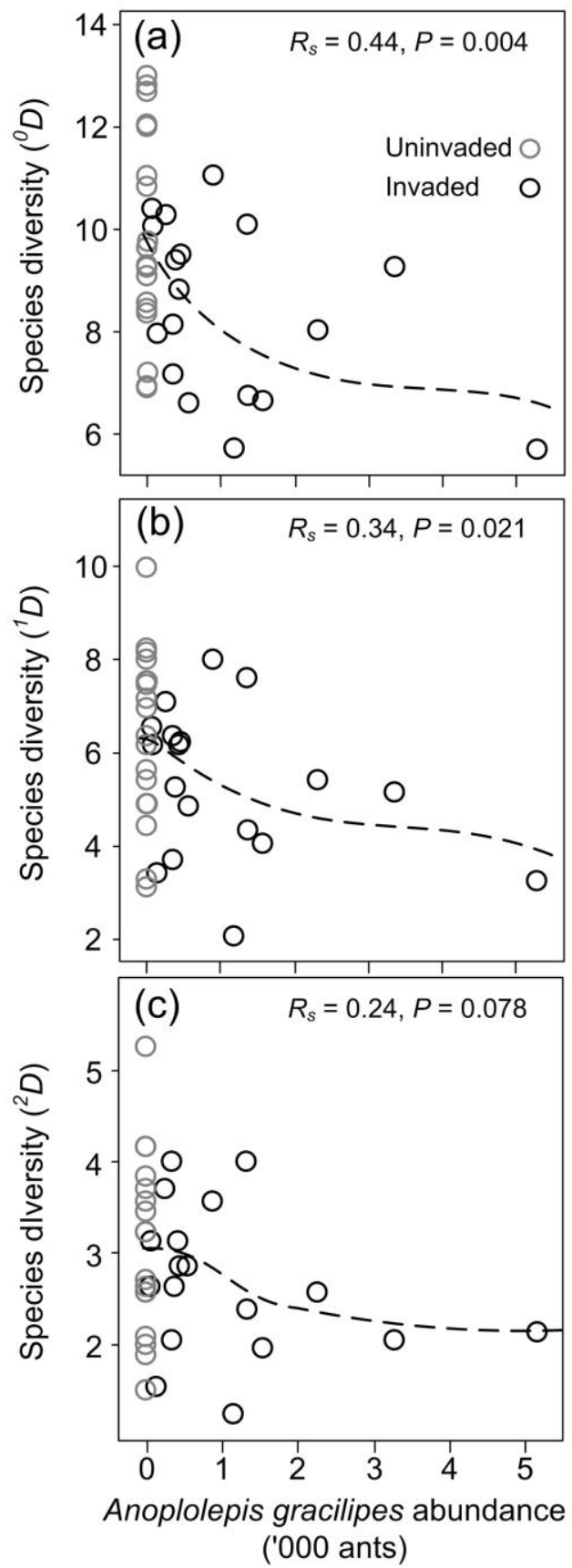

Fig. 4.5: Relationships between A. gracilipes abundance and native ant: (a) ${ }^{0} D$ (species richness); (b) ${ }^{1} D$ (exp [Shannon's H']); (c) ${ }^{2} D$ (1/Simpson's index). Dashed lines indicate the smoothed spline line of best fit with a span of 0.9. 


\section{Chapter 4}

Greater A. gracilipes abundance was associated with lower native ant species diversity for all measures of species diversity (Fig. 4.5), but the effect size and statistical significance of this relationship was lower for the measures that placed less importance on rare species $\left({ }^{0} D: S=11151, R_{S}=0.44, P=0.004 ;{ }^{1} D: S=10422, R_{S}=0.34, P=0.021\right.$;

$\left.{ }^{2} D: S=9647, R_{s}=0.24, P=0.078\right)$. This result mirrored our finding that species diversity did not differ between invaded and uninvaded plots when rare species were assigned less importance, and supports our finding that rare species are less likely to co-occur with $A$. gracilipes. The relationships between abundance and ant species diversity were not linear, and instead were best fit by lines that tended toward a sigmoidal function. These relationships suggest a saturation point for these measures.

\subsubsection{Are habitat characteristics associated with differences in Anoplolepis gracilipes abundance?}

None of the habitat characteristics we measured were significantly correlated with differences in A. gracilipes abundance. For all plots combined the best model included site as a fixed factor and canopy, Acacia and litter as independent variables. The best model explained $48 \%$ of the variation in the data, but no variables were statistically significant $\left(R^{2}=0.20\right.$, canopy: $F=1.02, P=0.324$, Acacia: $F=1.38, P=0.273$, litter: $F$ $=3.06, P=0.080$ ). For invaded plots only the best statistical model included site and Acacia, and explained $65 \%$ of the variation in the data, but again, no variables were statistically significant $\left(R^{2}=0.18\right.$, Acacia: $\left.F=0.94, P=0.44\right)$. The principal components analysis also did not reveal any differences between invaded and uninvaded plots for any of the habitat characteristics measured (Supplementary Fig. 4.3).

\subsection{Discussion}

Populations of invasive species often experience genetic bottlenecks that typically result in reduced genetic variation in the introduced population relative to the parent population (Sakai et al. 2001). Although introduced populations may persist in the short term, a lack of genetic variation may impede the potential for adaptive evolution in the longer term. We found that higher abundance of A. gracilipes was significantly positively correlated with most measures of genetic diversity, which is consistent with the 
evolutionary theoretical expectations that invasive populations with higher genetic diversity should experience greater invasion success (Sakai et al. 2001). Our study was conducted at the scale at which ants are likely to interact (nest clusters), rather than at the population scale, and the abundance we measured reflects momentary invasion success. The longer term evolutionary benefits of higher genetic diversity to A. gracilipes populations are difficult to predict. The immediate functional benefits of higher genetic diversity, at both the scale of the nest cluster and the population as a whole, as well as the underlying causes of the relationship between genetic diversity and abundance, and the relationship between abundance and ecological effects on the invaded community merit further consideration.

\subsubsection{The functional benefits of higher genetic diversity}

We found that most, but not all, measures of genetic diversity were correlated with higher abundance. Although allelic richness $\left({ }^{0} D\right)$ was not significantly correlated with higher abundance of A. gracilipes in Arnhem Land, the relationship between the two was positive and moderately large. Genotypic diversity and ${ }^{l} D$ genetic diversity were significantly positively correlated with higher $A$. gracilipes abundance, but ${ }^{2} D$ was significantly negatively correlated with higher abundance. The interpretation of the biological relevance of the latter statistic is problematic. Thus, further discussion focuses on genotypic diversity, because: a) it relates directly to individuals; b) may indicate whether multiple queens and males contribute to reproduction within the nest cluster; and c) may be interpreted in light of asexual reproduction, which has been suggested possibly contributes to the reproductive mode of A. gracilipes (Drescher et al. 2007; Heinze 2008).

Nest clusters of $A$. gracilipes could obtain functional benefits from higher genetic diversity in a number of ways. The intra-colonial benefits of higher genetic diversity to social insects include increased resistance to infection (Sherman et al. 1988; Shykoff and Schmid-Hempel 1991; Keller 1995; Tarpy 2003; Reber et al. 2008; Ugelvig et al. 2010), genetically based task specialization (Oldroyd and Fewell 2007), enhanced colony growth (Cole and Wiernasz 1999; Tarpy 2003), and higher productivity and fitness (Mattila and Seeley 2007). However experimental tests have found no relationship between short term task efficiency and genetic diversity (Rosset et al. 2005). It remains to be seen whether 


\section{Chapter 4}

the fine scale, momentary correlation between genetic diversity and abundance that we observed translates into invasion success at larger spatial and temporal scales. However, given the findings of studies of other ants, it seems likely that higher genetic diversity of the worker population may have positive short term effects on local nest clusters.

If there are functional benefits of higher genetic diversity to some clusters, clearly other nest clusters do not receive these benefits. How then might variation in genetic diversity affect the dynamics of the A. gracilipes population in Arnhem Land as a whole? Anoplolepis gracilipes populations have been known to vary in abundance temporally, significantly decline or collapse entirely (Lewis et al. 1976; Haines and Haines 1978b; Hill et al. 2003; Abbott 2006b; B. Hoffmann, personal observation). If genetic diversity drives abundance, meta-population dynamics might result in 'sink' populations (Pulliam 1988) of lower genetic diversity that do not persist. The fluid nature of the population structure of A. gracilipes in Arnhem Land, and lack of significant aggression between geographically distant nests (Chapter 3) also suggests that 'sink' nest clusters may be able to receive additional propagules from 'source' nest clusters. If the relationship is reversed, and abundance drives higher genetic diversity, larger propagules may have better chances of persistence. Longer term study of the correlations between abundance and genetic diversity, and the spatio-temporal dynamics of abundance in A. gracilipes would reveal whether this was this case in Arnhem Land.

Although our findings appear to be in contrast to the hypothesis that genetic bottlenecks may promote unicoloniality, and thus invasion success, which has been suggested for L. humile (Suarez et al. 1999; Tsutsui et al. 2000), the potential positive effects of a bottleneck may only occur at the introduction event. Subsequent to the initial introduction event, relatively higher genetic diversity may offer benefits to local nest clusters as outlined earlier. Ideally, to determine the effects of potential bottlenecks that may have affected the success of $A$. gracilipes in the invaded range we would need a comparison with the native range. Unfortunately the native range of A. gracilipes is unknown. Although its congeners are African, the ant is suspected to have Asian origins (Wetterer 2005), which is supported by some evidence (Drescher 2011; Sebastien et al. 2012). For example, the closest relatives of some endosymbionts of A. gracilipes or the hosts of those endosymbionts appear to be from Asia (Sebastien et al. 2012), and lower 
diversity at mitochondrial markers in Indo-Pacific populations suggests that these may have stemmed from populations in Asia (Drescher 2011). However, the entire range is not known and has not been sampled. Population divergence of A. gracilipes subsequent to introduction in Arnhem Land (Chapter 3) is also likely to have obscured evidence of a bottleneck that could have occurred on arrival. Thus, whether a bottleneck during the introduction event may have promoted unicoloniality in the Arnhem Land population is unknown.

\subsubsection{Mechanisms underlying the relationship between genetic diversity and abundance}

Variation in genetic diversity may be responsible for variation in the abundance of $A$. gracilipes in Arnhem Land, or it may be that more abundant populations are more genetically diverse because more individuals contribute to reproduction. In ant societies, census population size $(N c$ - workers together with reproductives) and effective population size ( $\mathrm{Ne}$ - individuals contributing to reproduction) are potentially decoupled, as reproduction often involves relatively few individuals, and these are not workers (Wilson 1963). We assumed this might be the case for A. gracilipes. However, our results do show that genotypic diversity and abundance are to some degree coupled. In addition, there is the suggestion that A. gracilipes workers may contribute to reproduction (Heinze 2008), in which case worker abundance and genetic diversity could be more closely coupled if more worker clones result in higher abundance. Ant colonies can also be more genetically diverse if more queens and / or males contribute to reproduction (i.e., multiple queens reproduce [polygyny], or queens mate with multiple males [polyandry]: Pamilo 1991). Although the number of queens in A. gracilipes nests in Arnhem Land varies (1 16 queens per nest: Chapter 2) and is often much higher (up to 300 queens per nest: Haines and Haines 1978a; Rao and Veeresh 1991), polygyny has not been investigated in the species. If polygyny makes a significant contribution to A. gracilipes genetic diversity, a decline in queen abundance may result in lower genetic diversity within the nest (or nest cluster). Clearly, further exploration of the dynamics of colony structure and reproductive mode of $A$. gracilipes are required to answer these questions. 


\section{Chapter 4}

An alternative hypothesis is that the correlation between abundance and genetic diversity in A. gracilipes in Arnhem Land is owing to invasion history. In this scenario, genetic drift could result in an association between genetic diversity and abundance as a result of successive bottlenecks during dispersal throughout Arnhem Land. Indeed, the population appears to be in the process of divergence into multiple colonies (Chapter 3), a process hypothesised to be driven by colony fragmentation and genetic drift (Drescher et al. 2010). If this is the case, older populations would likely be more genetically diverse than younger populations, as the younger populations would be increasingly bottlenecked. Although possible, this hypothesis is not consistent with the invasion history and dynamics of abundance observed among a number of A. gracilipes populations. In Arnhem Land, for example, when the ant was first detected it was most abundant near sites K6 and K7, while in all other areas it was in low abundance (Young et al. 2001). In addition, nest clusters that have been highly abundant suddenly decline in Arnhem Land (B. Hoffmann, unpublished data), and in Tokelau, younger populations often appear to be more abundant than older populations (Lester and Tavite 2004; Abbott et al. 2007; Chapter 5), although the situation is thought to be reversed on Christmas Island (Abbott 2006b). Regardless, this hypothesis would be difficult to test without more detailed knowledge of the invasion history of nest clusters within the population, which is also complicated by the potential of lag times obscuring detection of younger nest clusters if they were at low abundance.

\subsubsection{Density-dependent relationships between Anoplolepis gracilipes abundance and native ant community structure and diversity}

Frequently the effects of invasive ants on the resident ant communities are densitydependent (e.g., Ross et al. 1996; Le Breton et al. 2005; Krushelnycky and Gillespie 2008). Although the negative ecological effects of A. gracilipes are clearly densitydependent on ant communities (Hill et al. 2003; Abbott et al. 2007; Lester et al. 2009; Hoffmann and Saul 2010; Drescher et al. 2011; this study) and other invertebrates (McNatty et al. 2009; Boland et al. 2011), in some instances A. gracilipes is not able to reach sufficient abundance for negative ecological effects to occur (Abbott et al. 2007; Hoffmann and Saul 2010; Boland et al. 2011; Drescher et al. 2011; this study; Chapter 5). The effect of invading ants on the recipient ant community does not always result in a 
decline in species richness, as some species may increase in abundance while others decrease (Guénard and Dunn 2010). We used a combination of diversity measures, which revealed more than species richness alone regarding the nature of differences between communities. Species richness was not always lower in invaded plots than uninvaded plots. While rare species were less likely to occur with A. gracilipes in Arnhem Land, small, inconspicuous, insinuating species were commonly present. Other studies of $A$. gracilipes have found lower ant species richness in invaded sites (e.g., Sarty et al. 2007; Savage et al. 2009; Hoffmann and Saul 2010; Drescher et al. 2011), and that ecologically dominant species are less likely to co-occur with A. gracilipes (Hoffmann and Saul 2010). When A. gracilipes reaches high abundance it may cause more marked changes to ant community structure (Abbott et al. 2007; Lester et al. 2009). So while the absence of rare ant species when A. gracilipes is highly abundant may be of conservation concern, the patchy distribution and variable abundance of A. gracilipes in Arnhem Land may be unlikely to have significant effects on regional native ant diversity.

\subsubsection{Habitat characteristics may be less important than genetic diversity}

When viewed in a biogeographical context it is clear that many factors influence invasion success (Wilson et al. 2009a). Although many introduced populations experience a reduction in genetic diversity in the invaded range (e.g., Grapputo et al. 2005; Zayed et al. 2007; Dlugosch and Parker 2008), they may thrive if the new ecological conditions are more favourable than the native range (Sax and Brown 2000; Colautti et al. 2004; Moles et al. 2008). Alternatively, higher genetic diversity may be a more important contributor to invasion success where novel ecological conditions do not promote abundance. We suggest that this may be the case for A. gracilipes in Arnhem Land, where we found no association between the habitat characteristics we assessed and A. gracilipes abundance, yet we did find positive relationships between most measures of genetic diversity and abundance.

Of the habitat variables we measured, carbohydrate resources are perhaps the most important driver of A. gracilipes abundance elsewhere (O'Dowd et al. 1999; O'Dowd et al. 2003; Savage et al. 2009), and strongly influence ant abundance generally (Davidson 1997 and references therein). Thus, it was surprising that we found no effect of the 


\section{Chapter 4}

presence of Acacia on the abundance of A. gracilipes. The abundance of $A$. gracilipes in Arnhem Land appears very low compared to the ant on Christmas Island (our card counts ranged from 0 to 42, compared with a range of $\sim 14-136$ on Christmas Island, Abbott 2005), where A. gracilipes abundance is facilitated by honeydew-exuding scale insects (O'Dowd et al. 2003). The lesser abundance we observed may be owing to a lack of exploitation or availability of carbohydrate resources in Arnhem Land. Conversely, A. gracilipes may not have reached (or be able to reach) a minimum level of abundance required to monopolise exudate producing resources, as found in Technomyrmex albipes (Oliver et al. 2008). Regardless of the direction of the relationship between carbohydrate resources and abundance, our results suggest that in the absence of clear ecological drivers of abundance, genetic diversity may more likely to be associated with abundance. We do not deny that habitat characteristics, and particularly novel resources, often influence the abundance and ecological success of ants, but in this case the correlation with genetic diversity was much stronger than with the habitat variables we measured.

\subsection{Conclusion}

We found evidence of a positive association between measures of genetic diversity and the abundance of A. gracilipes in Arnhem Land. While higher genetic diversity may benefit individual nest clusters, the underlying mechanisms and the direction of the relationship between abundance and genetic diversity are unclear, and the implications for longer term invasion success on the wider population are difficult to predict. Although the effects of A. gracilipes on the invaded ant community in Arnhem Land are densitydependent, the variability in A. gracilipes abundance may be unlikely to have significant effects on regional native ant diversity over longer timescales. Finally, the relative importance of the relationship between genetic diversity and abundance may be contextdependent, may change over time, and be more obvious in the absence of highly favourable ecological characteristics. 


\section{Chapter 5. Population decline but increased distribution of an invasive ant genotype on a Pacific atoll}

\subsection{Abstract}

Populations of invasive species are often studied when their effects are perceived as a problem. Yet much can be gained by observing the dynamics of populations over longer time periods, particularly if multiple cryptic populations are present that differ in their ecological effects. In this study we revisit an invasion of the yellow crazy ant (Anoplolepis gracilipes) in the Tokelau archipelago to determine if the distribution and abundance of the ant has changed $\sim 7$ years after surveys completed in 2004 . We were particularly interested in whether populations of a previously identified invasive haplotype (D) had increased in distribution and abundance, as this haplotype was implicated in negative effects on the resident ant communities. Indeed, haplotype D populations have become more widespread since the initial survey, although this is more likely owing to new introductions or movement by humans, rather than intrinsic characteristics of the haplotype. We also found that although there has been no significant change in the abundance of A. gracilipes overall, haplotype D populations have significantly declined in abundance. Residents of the two Tokelau atolls where $A$. gracilipes has remained since 2004 no longer consider the ant to be a pest as they did seven years ago, when populations of this ant interfered with their food production and many other aspects of daily life. We observed no significant effects of A. gracilipes on the local ant community, which suggests that the ant is at a level of abundance below which significant negative ecological effects may occur. Population declines of invasive species are not infrequent, and understanding these population dynamics, particularly the underlying mechanisms promoting population declines or stabilisation, should be a high priority for invasion ecology. 


\section{Chapter 5}

\subsection{Introduction}

In invasion ecology, populations of invasive species are often sampled, and their effects described, when they are perceived as a problem (Strayer et al. 2006). These populations attract attention because of their high densities and negative effects on biodiversity and human populations. Invading populations are less often re-examined after long intervening periods (Strayer et al. 2006). It is often expected that invading populations that are already present in high numbers would persist and spread if left unchecked. Indeed, many studies have found that invading populations become more abundant and their impacts on the invaded community intensify over time (e.g., O'Dowd et al. 2003; Simberloff and Gibbons 2004; Hoffmann and Parr 2008). In other cases invading populations can expand or contract their range and abundance over time, and in some cases abundant populations can suffer local declines, or disappear entirely (e.g., Lewis et al. 1976; Morrison 2002; Hill et al. 2003; a number of studies reviewed by Simberloff and Gibbons 2004; Cooling et al. 2011). Thus, the predictive value of a single point-in-time study has limitations in relation to longer term invasion outcomes (Strayer et al. 2006).

In addition to changes occurring in population dynamics and ecological effects over time, the genetic nature of the population may affect invasion outcomes. For example, non-native populations of the reed grass Phragmites australis in North America have faster rates of spread than native populations. These cryptic non-native populations can only be differentiated from native populations by analysis of their genotypes (Saltonstall 2002). Similarly, the whitefly (Bemisia tabaci species complex) has a number of genetic biotypes, of which only two have attained pest status (Perring 2001). Identification of these genetic forms enables predictions regarding their spread and displacement of other genetic forms. Thus, genetic insights into the invading population can be an important aid to management if the abundance, spread and ecological effects of the invader vary according to the genotype. 
In 2004, Abbott et al. (2007) conducted surveys of the invasive yellow crazy ant Anoplolepis gracilipes on the Tokelau atolls. Anoplolepis gracilipes is one of the most widespread invasive ants (Holway et al. 2002), and is listed among a selection of 100 of the world's worst invasive species (Lowe et al. 2000). Abbott et al. (2007) found that populations of the ant on the Tokelau atolls belonged to one of two common and behaviourally distinct mitochondrial haplotypes (A and D). Haplotype D was associated with more abundant populations and strong negative effects on the resident ant community. On Nukunonu atoll, these haplotype D populations were found on the western islets while haplotype A populations were found on the eastern islets. This distribution appeared to reflect the ages of the populations, with the western, more abundant haplotype D populations likely to be younger as they occurred on the inhabited islets (Fig. 5.1; Lester and Tavite 2004; Abbott et al. 2007). The less abundant haplotype A populations on the eastern islets likely represented an older invasion (Lester and Tavite 2004; Abbott et al. 2007). These eastern populations appear to be in densities low enough to allow resident ant species to co-exist (Lester et al. 2009). Haplotype D populations were also found on one islet of Fakaofo atoll at high abundance. At the time A. gracilipes was absent from the third atoll, Atafu. Clearly, if there is a persistent (i.e., causative) association between the haplotype, the abundance, and ecological effects of the ant, knowledge of the genetic identity of $A$. gracilipes populations would aid in the prediction of harmful effects.

In this study we revisited the A. gracilipes invasion on Tokelau, focussing in particular on differences in the distribution, abundance and effects of populations of the different haplotypes on resident ant communities. We addressed three specific questions: 1) Has the distribution of A. gracilipes among the three atolls changed? 2) Has the abundance of A. gracilipes changed on Nukunonu atoll? 3) Is there still an association between $A$. gracilipes abundance and effects on the invaded ant communities on Nukunonu atoll? 


\section{Chapter 5}

\subsection{Methods}

\subsubsection{Study site}

The atolls of the Tokelau archipelago lie in the humid tropics, $\sim 500 \mathrm{~km}$ north of Samoa. The atolls experience a wet tropical rainforest climate with a mean annual temperature of $28^{\circ} \mathrm{C}$, and mean annual rainfall of $>3000 \mathrm{~mm}$ (Mueller-Dombois and Fosberg 1998). Tokelau is made up of three atolls, Fakaofo, Nukunonu and Atafu, each with 31 - 58 low-lying coral islets encircling shallow lagoons. When Abbott et al. (2007) first studied A. gracilipes on Tokelau in 2004 the ant was observed on only two atolls: Nukunonu, where seven of about 42 islets were invaded, and Fakaofo, where two of about 51 islets were invaded. By 2006, the abundance of A. gracilipes appeared to be diminished, perhaps owing to the effects of Cyclone Percy in 2005 (K. Abbott, personal observation), but the ants remained in high densities in some areas. In 2006 poisoning was undertaken on the inhabited islets at Fakaofo and Nukunonu. These islets were Fenua Fala at Fakaofo atoll (at the time the only islet on which A. gracilipes was present at Fakaofo), and on Motuhaga and Nukunonu islets at Nukunonu atoll. Since then $A$. gracilipes has also been reported on Atafu atoll. Data for the current study were collected during a single visit to all three atolls in September - October 2011, which was seasonally comparable to the timeframe the Abbott et al. (2007) data were collected. The Tokelau atolls are serviced from Apia, Samoa by one of three vessels every 7 - 14 days. Apart from the occasional yacht these service vessels are the only apparent source of ants present on the atolls. Although distant from Samoa, the frequency of ship transport and biosecurity challenges mean that the likelihood of ant invasion is high.

\subsubsection{Ecological surveys}

To determine if A. gracilipes distribution has changed over the intervening years since the Abbott et al. (2007) study, we conducted hand searches on all islets at Nukunonu and Atafu atolls (approximately 42 and 40 islets respectively). Logistical constraints made it possible to survey Fakaofo only briefly (Fale islet). 
We conducted an ecological survey to assess the abundance of A. gracilipes, and ant community structure on nine islets on Nukunonu atoll (Fig. 5.1). Sites were selected based on the earlier presence of distinct genetic haplotypes (Abbott et al. 2007). These islets were Nukunonu, Motuhaga, Te Puka i Mua (all haplotype D), Te Fala, Pukapuka, Tokelau, Fenua Loa / Lalo (all haplotype A), Te Nonu and Te Palaoa (both previously uninvaded by A. gracilipes). Following the methods of earlier studies (Lester and Tavite 2004; Abbott et al. 2007), we selected two $15 \times 15 \mathrm{~m}$ plots on each islet that were at least $100 \mathrm{~m}$ distant from each other. Pitfall traps were used to examine species richness and ant community composition, and assess the relative abundance of A. gracilipes. At each plot we installed five haphazardly placed pitfall traps ( $75 \mathrm{~mm}$ diameter). Traps were 1/3 filled with propylene glycol and left for 24 hours, after which all ants collected were counted, identified and named to species level where possible using the earlier collections of Abbott et al. (2007) and Lester et al. (2009) as a reference. Specimens of all species were retained at Victoria University of Wellington (VUW).

In addition to $A$. gracilipes counts in pitfall traps, on Nukunonu we also measured the abundance of $A$. gracilipes at each site based on forager activity, as described in Chapter 3. Briefly, this method uses a count of the number of ants crossing a laminated card in a $30 \mathrm{~s}$ period (Green et al. 2004). At each site we measured A. gracilipes activity at 11 stations spaced at $5 \mathrm{~m}$ intervals along three replicate $50 \mathrm{~m}$ transects spaced $10 \mathrm{~m}$ apart. Counts at all stations within each transect were summed, and the mean value of the three replicate transects was used as an index of relative abundance between sites. We also conducted card counts of A. gracilipes on Atafu atoll, and mapped the extent of the large invasion we found there using a GPS (Garmin).

We also interviewed representatives of the local communities on each atoll regarding their perceptions of the A. gracilipes invasion. We asked whether the people had observed changes in A. gracilipes distribution or abundance over time, and if they perceived the ant to be a pest. 


\section{Chapter 5}

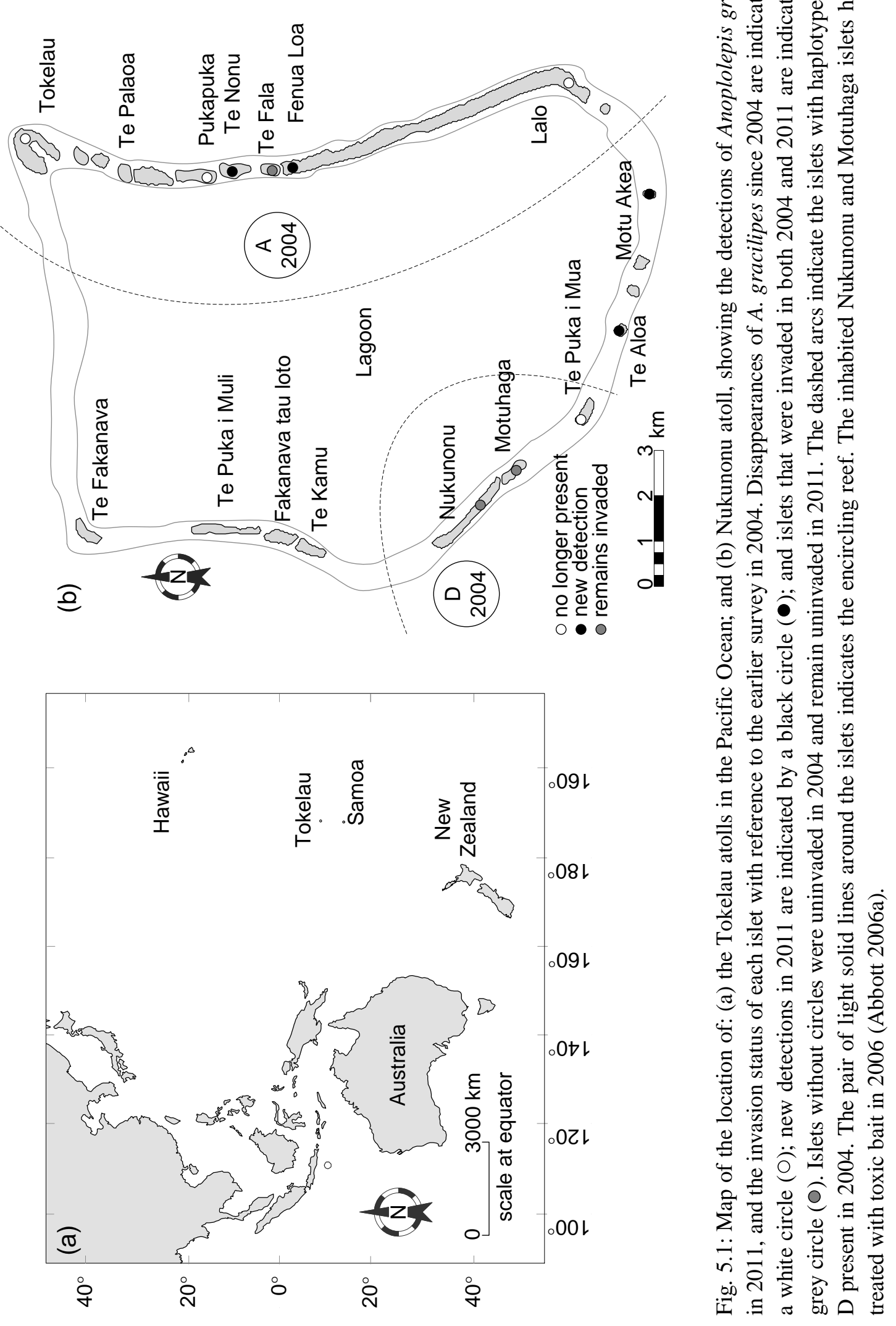




\subsubsection{Molecular analyses}

To ascertain the prevalence of $A$. gracilipes haplotypes we sequenced one or two individuals from each collection (pitfall traps and hand collections) on Nukunonu atoll (19 samples). In addition, we sequenced A. gracilipes from Fakaofo (2 samples) and Atafu (5 samples) to determine the current distributions of populations with different haplotypes throughout Tokelau. We also amplified DNA extractions of ants from Tokelau (2 samples), Tonga (2 samples), and Samoa (4 samples) that had previously been sequenced for cytochrome b (cyt b) by Abbott et al. (2007) to confirm that our COI haplotypes were congruent with the haplotypes found by Abbott et al. (2007), and to enable comparison between studies.

Ants collected for genetic analysis were stored in $\sim 95 \%$ ethanol at $4^{\circ} \mathrm{C}$. We extracted DNA using a modified Chelex protocol (Sepp et al. 1994). Individual workers were placed in microcentrifuge tubes, ground with sterile plastic pestles, and $150 \mu \mathrm{l}$ of a $10 \%$ w/v Chelex-100 resin solution was added. The tubes were centrifuged briefly, boiled for 15 minutes in a water bath, chilled on ice for five minutes, and centrifuged at 15,000 $\times g$ for 15 minutes at $4^{\circ} \mathrm{C}$. The supernatant containing DNA was stored at $4^{\circ} \mathrm{C}$.

We used mitochondrial markers to determine if multiple mitochondrial haplotypes were present, targeting the COI (cytochrome oxidase I) region. Each $15 \mu 1$ PCR consisted of $20 \mathrm{ng}$ template DNA, 10× PCR Buffer, $0.4 \mu \mathrm{g} \mathrm{mL} \mathrm{m}^{-1}$ of bovine serum albumin (BSA), $1.5 \mathrm{mM} \mathrm{MgCl} 2,0.2 \mathrm{mM}$ of each dNTP, $0.4 \mathrm{mM}$ forward and reverse primer (CI-13 and CI-14, Quek et al. 2004), and 0.3 U of Taq DNA Polymerase (either Bioline BIOTAQ, Invitrogen Platinum ${ }^{\circledR}$ or New England Biolabs). Thermal cycling consisted of an initial denaturation at $94^{\circ} \mathrm{C}$ for $2 \mathrm{~min}$, followed by 40 cycles of denaturation at $94^{\circ} \mathrm{C}$ for $30 \mathrm{~s}$, annealing at $40^{\circ} \mathrm{C}$ for $40 \mathrm{~s}$, extension at $72^{\circ} \mathrm{C}$ for $1 \mathrm{~min}$, and a final extension at $72^{\circ} \mathrm{C}$ for 10 min. Amplified products were purified using ExoSAP-IT (US Biochemicals) and sequenced on a 3730 Genetic Analyser (Applied Biosystems). 


\section{Chapter 5}

We checked and edited the sequences using ClustalW (Thompson et al. 1994) implemented in MEGA4 (Tamura et al. 2007), and translated our aligned COI sequences of 533 base pairs to detect any premature stop codons that could indicate a sequence of non-mitochondrial origin. A BLAST search in GenBank confirmed the authenticity of our sequences as A. gracilipes. We constructed a parsimony haplotype network using TCS v 1.21 (Clement et al. 2000) to determine the relationships among the sequences. We have deposited the cyt b haplotypes of Abbott et al. (2007) in GenBank together with our COI haplotypes (Accessions pending).

\subsubsection{Statistical analyses}

To determine if the abundance of A. gracilipes in 2011 differed from 2004, and if haplotype D populations differed in abundance from haplotype A populations, we fitted a generalised linear model in R v 2.14 (Ihaka and Gentleman 1996; R Development Core Team 2011). We included only those plots where A. gracilipes was detected in 2004 or 2011. To account for over-dispersion of the data, we used a negative binomial distribution with a $\log \operatorname{link}$ function and theta $=1$. Anoplolepis gracilipes abundance was the response variable, and haplotype nested within sampling year were the predictor variables.

We also used generalised linear models to determine if there still existed an association between the abundance of yellow crazy ants, the haplotype of the population, and the effects on species diversity in the invaded community. Again, we used a generalised linear model with a negative binomial distribution, log link function and theta $=1$. The response variable was species richness, and haplotype identity and A. gracilipes abundance were predictor variables. We included data for 2011 only and ran models with the interaction term, main effects and single predictors. We used AIC scores (Akaike 1973; Burnham and Anderson 2002) to determine the model that best fit the data, using AICc scores generated by the 'AICcmodavg' package (Mazerolle 2011) in R.

To assess differences in ant community composition between sampling years, and in 2011 between invaded and uninvaded plots, and between different haplotypes we used non-metric Multi-Dimensional Scaling (MDS) and PERMANOVA+ implemented in PRIMER v 6.1.11 (Clarke and Gorley 2006). Data for A. gracilipes were excluded from 
the analysis, along with two plots where no ants were recorded (Tokelau [2011] and Te Palaoa [2004]). We log-transformed data to even out the effects of rare and abundant species, and used the Bray-Curtis index as a similarity measure as recommended by Clarke and Warwick (2001). The MDS was run using 1000 iterations with a Kruskal stress formula 1 and a minimum stress of 0.01 . The significance of differences between groups was tested with PERMANOVA+ run over 99999 permutations (Anderson et al. 2008).

\subsection{Results}

\subsubsection{Changes in the distribution of Anoplolepis gracilipes in Tokelau}

Since 2004 the distribution of A. gracilipes has increased on the Tokelau atolls, and the majority of new detections were of haplotype D. On Fakaofo atoll we were able to visit only one of the two inhabited islets. Along with the known presence of A. gracilipes on the first inhabited islet (Fenua Fala), the second inhabited islet (Fale) that was not invaded by A. gracilipes in 2004 was found to be invaded in 2011 with a haplotype D population. Of the 40 islets searched on Atafu atoll, the sole inhabited islet, which was not invaded by A. gracilipes in 2004, was found to be invaded in 2011, also with a haplotype D population. The major Atafu invasion covered an area of approximately 37 Ha. At Nukunonu atoll, where we searched 42 islets, we found a number of differences in the distribution of A. gracilipes compared to 2004 (Table 5.1). Although we were unable to find A. gracilipes on a number of islets where it was abundant in 2004, populations of the ant were found on new islets in 2011, and populations remained on other islets (both haplotypes; Fig. 5.1). The new invasions at Nukunonu atoll were primarily of haplotype $\mathrm{D}$ (three islets), along with one invasion of haplotype A (Table 5.1). The new invasion on Motu Akea at Nukunonu atoll also included a single ant with a new haplotype (e), which was closely related to haplotype D (Table 5.1, Fig. 5.2). The populations that had apparently disappeared since 2004 included the most abundant haplotype D population (Te Puka i Mua islet: Fig. 5.1, Table 5.1), and three haplotype A populations (Lalo, Pukapuka and Tokelau islets: Fig. 5.1, Table 5.1). Although we were unable to definitively conclude that $A$. gracilipes was locally extinct from these islets, their previously extremely high abundance had substantially decreased to undetectable levels. 


\section{Chapter 5}

All the populations that have remained on Nukunonu atoll since 2004 were of the same haplotype as detected in the Abbott et al. (2007) study. However, the definite pattern of haplotype D populations occurring on the western islets and haplotype A populations occurring on the eastern islets (Abbott et al. 2007) was no longer present, as the newly found eastern Te Nonu islet population was of haplotype D. New invasions were more often of haplotype D.

Table 5.1: Comparison of the genetic haplotypes and total pitfall trap abundance per site of Anoplolepis gracilipes on invaded islets on Nukunonu Atoll, Tokelau in 2004 (Abbott et al. 2007) and 2011. In addition, for 2011 we included card counts of ant activity. Dashes indicate the islet was not invaded. Zero values for abundance indicates non-detection in pitfall traps rather than absence, as the presence of the ant was confirmed at the site. The abundance of A. gracilipes on Motu Akea and Te Afua atolls was not assessed as we had insufficient time for pitfall trapping or card counts after the ants were detected. However, the ants were difficult to find on these two islets and thus abundance was assumed to be very low.

\begin{tabular}{lcccccc}
\hline \multicolumn{1}{c}{ Islet } & \multicolumn{2}{c}{ Haplotype } & & \multicolumn{2}{c}{ Abundance } & Card \\
\cline { 2 - 3 } \cline { 5 - 6 } & $\mathbf{2 0 0 4}$ & $\mathbf{2 0 1 1}$ & & $\mathbf{2 0 0 4}$ & $\mathbf{2 0 1 1}$ & Count 2011 \\
\hline Te Nonu & - & $\mathrm{D}$ & & - & 1937 & 63 \\
Te Puka i Mua & $\mathrm{D}$ & - & & 6776 & - & - \\
Nukunonu & $\mathrm{D}$ & $\mathrm{D}$ & & 108 & 0 & 0 \\
Motuhaga & $\mathrm{D}$ & $\mathrm{D}$ & & 405 & 8 & 0 \\
Te Fala & $\mathrm{A}$ & $\mathrm{A}$ & & 379 & 298 & 23 \\
Pukapuka & $\mathrm{A}$ & - & & 9 & - & - \\
Tokelau & $\mathrm{A}$ & - & & 71 & - & - \\
Fenua Loa & - & $\mathrm{A}$ & & - & 76 & 18 \\
Lalo & $\mathrm{A}$ & - & & 934 & - & - \\
Motu Akea & - & $\mathrm{D}$ e & & - & NA & NA \\
Te Afua & - & $\mathrm{D}$ & - & NA & NA \\
\hline
\end{tabular}




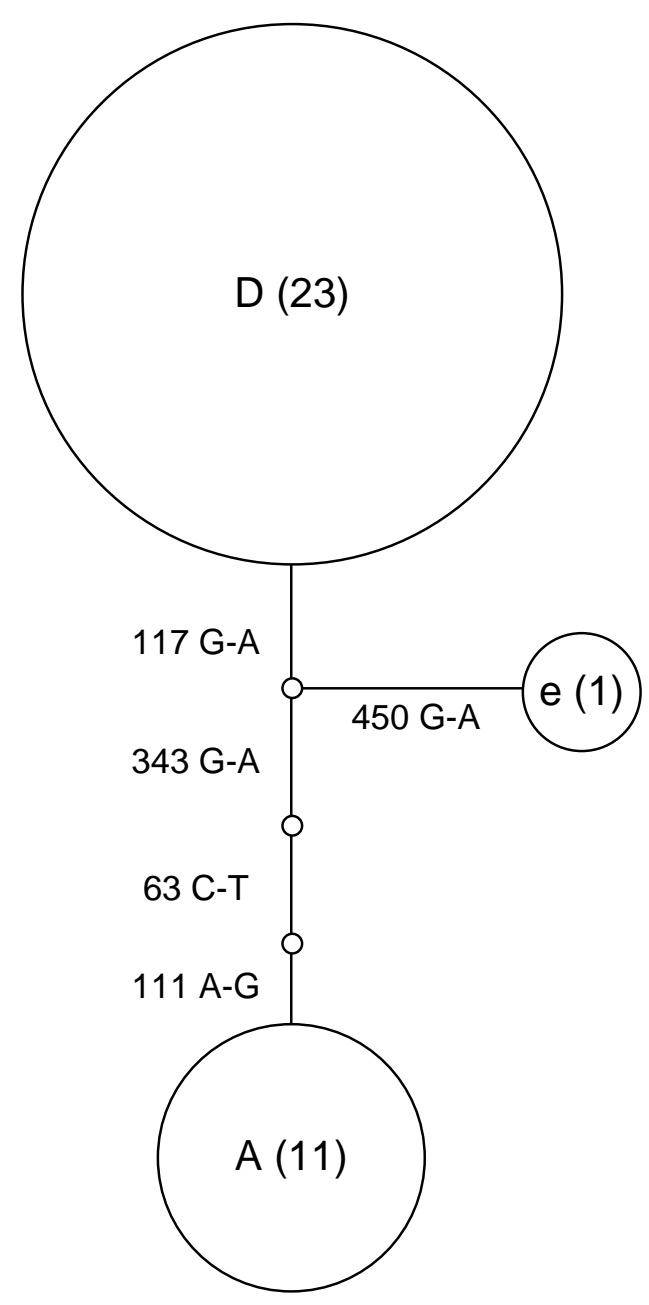

Fig. 5.2: Parsimony network describing the relationships between A. gracilipes COI haplotypes detected in Tokelau in 2004 and 2011. The number of samples included for each haplotype is given in brackets. Nucleotide substitution differences between haplotypes and their position in the sequence fragment are indicated. Table 5.1 details the islets on which the haplotypes were found. All substitutions were synonymous. Also included in the haplotype network were sequences using DNA extractions of 2004 samples from Tokelau, Samoa and Tonga for haplotype A (3 sequences), and Samoa and Tokelau for haplotype D (4 sequences), and 2011 samples from Atafu and Fakaofo (haplotype D, 7 sequences). The sizes of the circles reflect the number of sequences for the given haplotype. 


\section{Chapter 5}

5.4.2 Changes in the abundance of Anoplolepis gracilipes on Nukunonu atoll

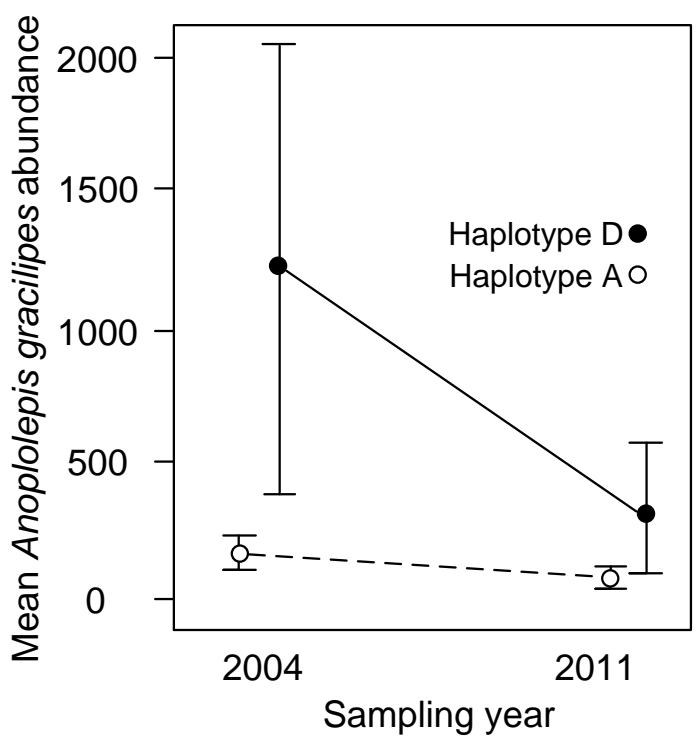

Fig. 5.3: Interaction plot comparing differences in mean pitfall trap abundance (per plot \pm SE) of A. gracilipes haplotype A (open circles) and D (closed circles) in 2004 and 2011 on Nukunonu atoll, Tokelau. The differences in abundance were not significant between sampling years (GLM: estimate $=-0.623, t=-0.681, P=0.503$ ), or between haplotypes in 2011 (GLM: estimate $=1.243, t=1.294, P=0.210$ ) but haplotype $\mathrm{D}$ was significantly more abundant in 2004 (GLM: estimate $=1.943, t=2.421, P=0.025$ ).

While the overall abundance of A. gracilipes at Nukunonu atoll appeared to be lower than in 2004, only the haplotype D populations had declined significantly (Fig. 5.3 \& Fig. 5.4, Table 5.1). In 2011 the mean abundance of A. gracilipes per pitfall trap among haplotype D islets was $64.8 \pm 23.2 \mathrm{SE}$ ( $n=30,3$ islets) compared to 2004 , when haplotype D islands had 243.0 $\pm 112.6 \mathrm{SE}$ ants per pitfall trap $(n=30,3$ islets: Abbott $e t$ al. 2007). Among haplotype A islets, mean pitfall trap abundance of A. gracilipes was 18.7 \pm 4.7 SE in 2011 ( $n=20,2$ islets), and $34.8 \pm 8.3 \mathrm{SE}$ ants per pitfall trap in $2004(n$ = 40, 4 islets: Abbott et al. 2007). Pitfall trap abundances were highest for the new haplotype D invasion on Te Nonu islet (Table 5.1). Although the differences in pitfall trap abundance were not statistically significant between sampling years (GLM: estimate = 0.623, $t=-0.681, P=0.503$ ), or between haplotypes in 2011 (Fig. 5.3; GLM: estimate $=$ 1.243, $t=1.294, P=0.210$ ), haplotype D abundance had significantly declined since 2004 (Fig. 5.3; GLM: estimate $=1.943, t=2.421, P=0.025$ ). 


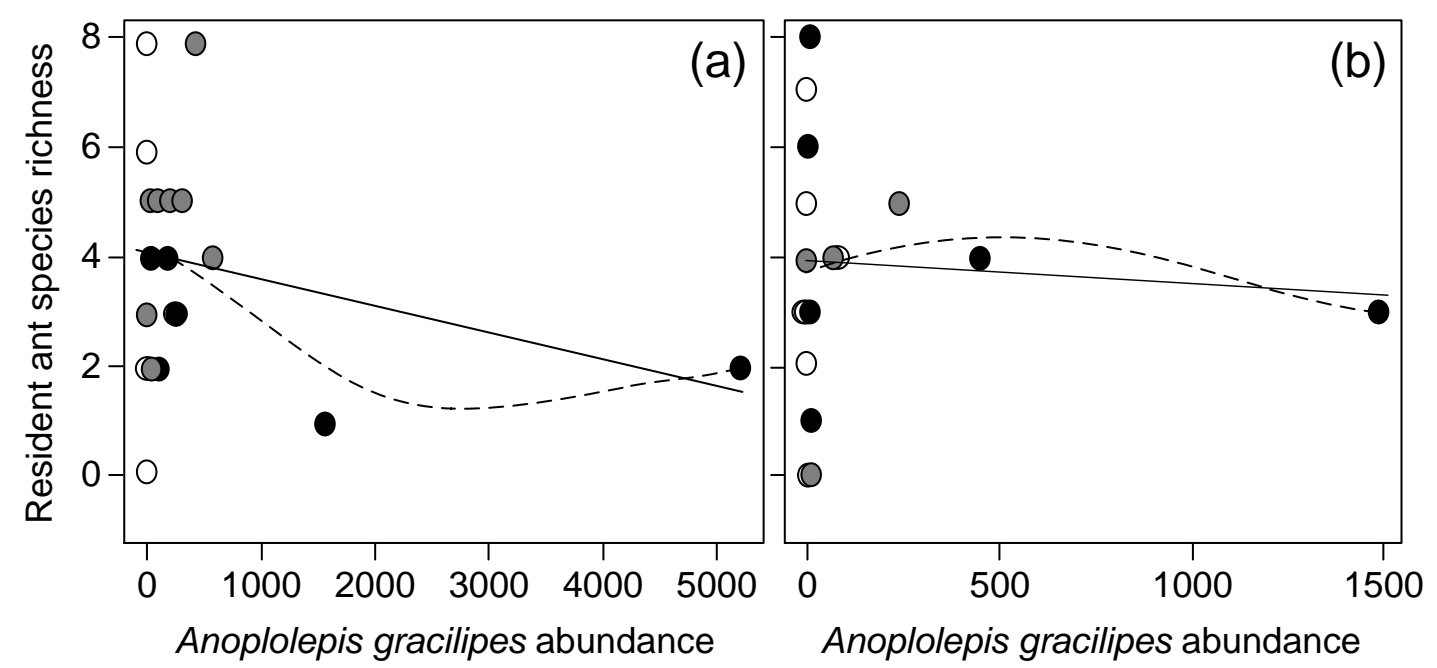

Fig. 5.4: The relationships between A. gracilipes abundance (total number of ants per invaded plot) and resident ant species richness in: (a) 2004; and (b) 2011. Dashed lines indicate the smoothed spline line of best fit with a span of 1 , and solid lines indicate the line of best fit under the assumptions of a linear model for data from invaded plots only. Plots with haplotype D ants are indicated by black circles $(\bullet)$, haplotype A by grey circles $(0)$, and uninvaded plots by open circles (O). In 2011 we found no relationship between the abundance of $A$. gracilipes, or haplotype $\mathrm{D}$, and species richness of the invaded ant community (GLM: estimate $=0.2485, t=0.608, P=0.560$ ).

Our card counts ranged from 0 to 63 at Nukunonu atoll, and were highly correlated with pitfall trap counts for each plot (Spearman's rank correlation $R_{s}=0.83, S=27.9, P=$ 0.001). Both card counts and pitfall trap abundances were highest for the new haplotype D invasion on Te Nonu in Nukunonu (Table 5.1). The card counts for the 37 Ha invasion of haplotype D on Atafu atoll were 20,52 and 63. The lowest card count at Atafu atoll was at a fuel storage area where local residents first noticed the ants. 


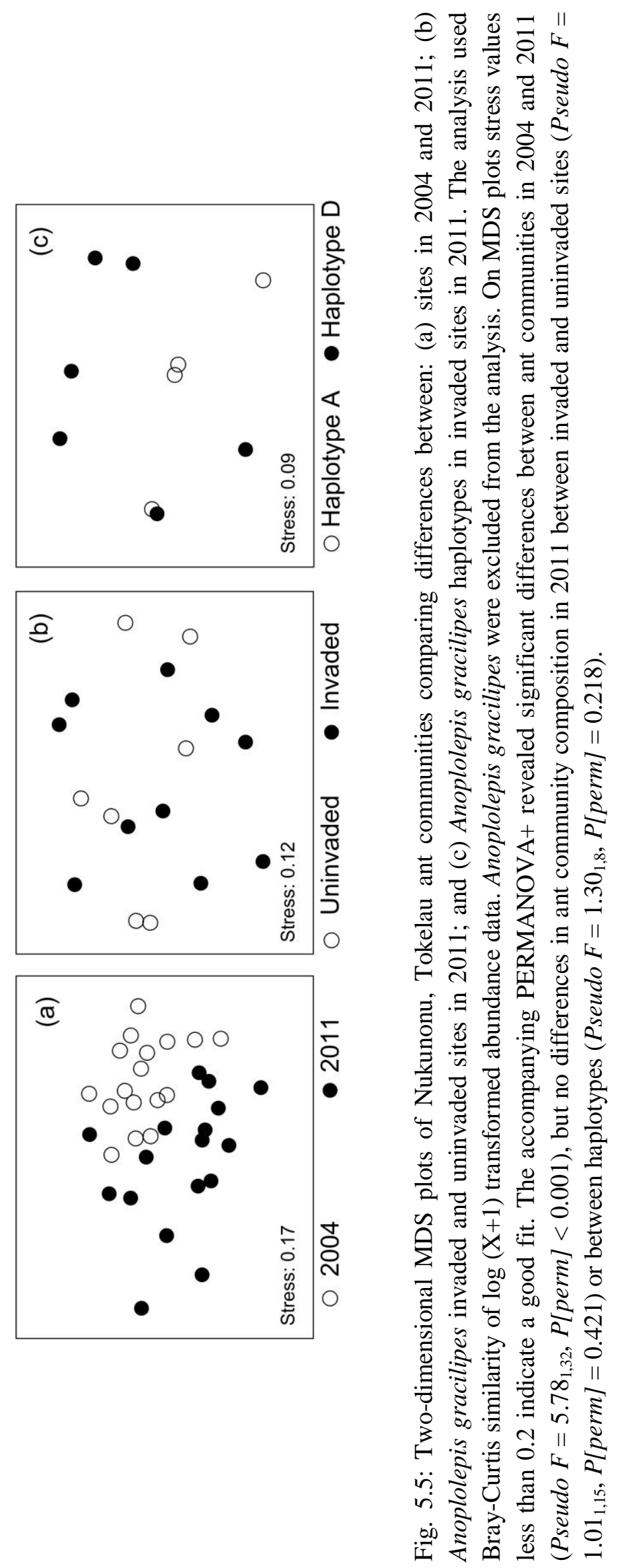




\subsubsection{The association between Anoplolepis gracilipes abundance and effects on the resident ant communities}

Excluding A. gracilipes we collected a total of 19 ant species in our pitfall traps (Table 5.2), compared to 16 species collected by Abbott et al. (2007). In 2011 species richness per plot ranged from 0 to 8 species (mean \pm SE: $3.8 \pm 0.7$ species in A. gracilipes invaded sites and $3.4 \pm 0.7$ species in uninvaded sites). Mean abundances of ants other than $A$. gracilipes ranged from 0 to 143 ants per plot (mean \pm SE: $30 \pm 15.2$ ants in A. gracilipes invaded sites and $18 \pm 7.8$ ants in uninvaded sites).

We found no significant relationship between haplotype or A. gracilipes abundance and species richness of the resident ant community. The generalised linear model with the lowest AICc score included haplotype as a main effect (AICc $=59.01$; GLM: estimate $=$ $0.2485, t=0.608, P=0.560)$. The next best fitting models included either A. gracilipes abundance $(\mathrm{AICc}=59.10)$, or haplotype and A. gracilipes abundance as main effects $(\mathrm{AICc}=64.97)$. In all the models no effects were statistically significant.

Two-dimensional MDS and PERMANOVA+ also revealed no significant differences in ant community composition in 2011 between A. gracilipes invaded and uninvaded sites (Pseudo $F=1.01_{1,15}$, P $[$ perm $\left.]=0.421\right)$ or between haplotypes $\left(\right.$ Pseudo $F=1.30_{1,8}$, $P[$ perm $]=0.218)$. The PERMANOVA+ analysis did however, reveal significant differences between ant communities in 2004 and 2011 (Pseudo $F=5.78_{1,32}, P[$ perm $]<$ 0.001; Fig. 5.5), which is consistent with a high rate of species turnover. PERMDISP analysis found that dispersions were homogeneous in 2011 between A. gracilipes invaded and uninvaded sites (Pseudo $F=0.672_{1,15}, P[$ perm $]=0.429$ ) and between haplotypes (Pseudo $F=2.44_{1,8}, P[$ perm $\left.]=0.214\right)$, but there were significant differences in dispersion between ant communities in 2004 and 2011 (Pseudo $F=7.48_{1,32}$, P[perm] $=$ 0.011 ), which indicates differences between years was specifically owing to differences in dispersion among sites. 


\section{Chapter 5}

Table 5.2: Comparison of the ant species caught in pitfall traps on Nukunonu Atoll, Tokelau in 2004 (Abbott et al. 2007) and 2011, and the number of islets on which they found.

\begin{tabular}{|c|c|c|c|c|}
\hline \multirow[t]{2}{*}{ Species } & \multicolumn{2}{|c|}{$\begin{array}{c}\text { A. gracilipes invaded } \\
\text { islets }\end{array}$} & \multicolumn{2}{|c|}{$\begin{array}{c}\text { Islets not invaded by } A \text {. } \\
\text { gracilipes }\end{array}$} \\
\hline & $\begin{array}{c}2004 \\
\text { (7 islets) } \\
\end{array}$ & $\begin{array}{c}2011 \\
\text { (7 islets) } \\
\end{array}$ & $\begin{array}{c}2004 \\
\text { (2 islets) } \\
\end{array}$ & $\begin{array}{c}2011 \\
\text { (4 islets) }\end{array}$ \\
\hline Anochetus graeffei & 1 & 3 & 2 & 2 \\
\hline Anoplolepis gracilipes & 7 & 5 & & \\
\hline Cardiocondyla nuda & 2 & 2 & & \\
\hline Iridomyrmex anceps & & 1 & & \\
\hline Monomorium floricola & 3 & 5 & & 1 \\
\hline Monomorium minutum & 1 & & & \\
\hline Paratrechina bourbonica & & 3 & & \\
\hline Paratrechina longicornis & & 1 & & 2 \\
\hline Paratrechina vaga & 2 & & 1 & 2 \\
\hline Pheidole fervens & 6 & & 2 & \\
\hline Pheidole oceanica & 4 & 1 & 1 & 2 \\
\hline Pheidole sexspinosa & 4 & 2 & & 2 \\
\hline Pheidole umbonata & 3 & & 2 & \\
\hline Pheidole sp. & & 2 & & 1 \\
\hline Ponera sp. & & & & 1 \\
\hline Pyramica membranifera & 1 & & & \\
\hline Rogeria stigmatica & 2 & & 1 & \\
\hline Strumigenys godeffroyi & 1 & & & \\
\hline Tapinoma melanocephalum & & 3 & & 2 \\
\hline Tapinoma minutum & & 2 & & 1 \\
\hline Tetramorium bicarinatum & & 2 & 1 & 2 \\
\hline Tetramorium lanuginosum & 5 & 4 & 1 & 2 \\
\hline Tetramorium simillimum & 2 & 2 & 1 & \\
\hline Tetramorium tonganum & 1 & 1 & 1 & \\
\hline Tetramorium sp. & & & & 1 \\
\hline
\end{tabular}

\subsubsection{Tokelau residents perceptions of the Anoplolepis gracilipes invasion}

The perceptions of the people of Tokelau were consistent with our ecological findings regarding the distribution and abundance of A. gracilipes. In 2003 and 2004 residents considered A. gracilipes to be a major pest. This ant had a devastating effect on crop production, irritated livestock, and interrupted the sleep of local residents by crawling over them during the 
night. Residents attempted to halt the spread and limit the abundance of the ant by burning of trees (Lester and Tavite 2004). In 2011 the residents of Nukunonu atoll considered that the abundance of $A$. gracilipes had decreased markedly since 2004 and they no longer considered the ant a serious problem. Nor did they report variations in abundance throughout the year. Management via poisoning had taken place in 2006 on inhabited islets at Nukunonu and Fakaofo atolls (Abbott 2006a). However, a cyclone in late 2005 shortly before poisoning was also attributed by the residents to be responsible for a significant reduction in A. gracilipes abundance.

At Fakaofo atoll residents were similarly unconcerned with the abundance of the ant on the islet that had been initially invaded (Fenua Fale), as it had declined substantially. However, people were concerned with the new invasion on the islet of Fale, where they reported the ant was a nuisance around outdoor kitchens. It was uncertain when $A$. gracilipes was first detected on Fale, but it was some months rather than years prior to the current study.

Since the 2004 study, A. gracilipes has been observed on the inhabited islet at Atafu atoll. The ant first became apparent to residents in 2008 around a fuel storage area, had spread from there to cover approximately $37 \mathrm{Ha}$ bordering on the village, and was also found at low abundance in a garden within the village. Many residents and the Atafu village council expressed serious concerns about the threat of the ant encroaching on the village, and were eager for management action.

There is no doubt that the supply vessels are a major source of ant species to the atolls. During our voyage from Samoa, we observed seven species of ants on the vessel These ant species were all later found to be resident on Tokelau, and included Tetramorium bicarinatum, Paratrechina bourbonica, P. vaga, $P$ longicornis, Tapinoma melanocephalum, Cardiocondyla nuda and Monomorium floricola. No arrivals on the atolls during our visit were subjected to a quarantine process, and no biosecurity checks were in place in Apia prior to our departure for Tokelau. 


\section{Chapter 5}

\subsection{Discussion}

Although the effects of invasive species are often most severe when the invading population is in high numbers (Ehrlich 1986; Crawley 1987; Mack et al. 2000), their abundance and ecological effects clearly do vary temporally, and population declines or collapses occasionally occur (e.g., Lewis et al. 1976; Morrison 2002; Hill et al. 2003; a number of studies reviewed by Simberloff and Gibbons 2004; Cooling et al. 2011). The distribution of $A$. gracilipes and the abundance of haplotype D populations in Tokelau in 2011 differed substantially from 2004. Although haplotype D populations were responsible for most new invasions between 2004 and 2011, these populations were not significantly more abundant than those of haplotype A at Nukunonu atoll. Thus, the pattern of numerical dominance of haplotype D populations was no longer present, and there no longer existed an association between haplotype D populations and effects on the invaded ant communities. Indeed, the resident ant communities invaded by A. gracilipes did not differ from the uninvaded communities in structure or species richness, and other ants often suffer the earliest and most severe effects of invasive ants (Holway et al. 2002). The perceptions of the local Tokelauan people also indicated a substantial decline in $A$. gracilipes abundance and residents were no longer concerned about the ant when it was in low abundance.

\subsubsection{Changes in Anoplolepis gracilipes distribution on Tokelau}

The distribution of $A$. gracilipes among the atolls of Tokelau has increased to all three atolls, and although the abundance of haplotype D populations had declined, this haplotype featured in the majority of new detections. Although we were unable to sample ants from Samoa (the most likely origin of ants in Tokelau), haplotype D was present in Samoa and Tokelau in 2004 (Abbott et al. 2007). While we found a single occurrence of a new haplotype (e), this new haplotype may or may not be associated with a new invasion, as Abbott et al. (2007) also found rare haplotypes among the populations they sampled. However, we cannot be certain whether the new detections were due to arrivals from within Tokelau or from Samoa. Indeed, the ant communities at Nukunonu atoll are strikingly different to those of 2004, indicating high species turnover, and we found seven ant species in our brief survey of the ship to Tokelau. In 2002 three species (Monomorium 
minutum, $P$. vaga, and T. bicarinatum) were observed in only four small potted plants being moved onto the supply vessel to Nukunonu atoll (Lester and Tavite 2004). While all the species found on the supply vessels were all found in our pitfall traps, the risk of re-invasion (and invasion of new species) is clearly high as there were no biosecurity measures in place either before our departure from Samoa or on arrival in Tokelau.

Haplotype D populations seem more likely to feature in new detections because they were present on the inhabited islets in Tokelau, and are thus more likely to be transported. An alternative hypothesis, that haplotype D populations have displaced other ants due to enhanced competitive ability, seems less likely given the decline in abundance of these populations.

\subsubsection{Density-dependent effects of Anoplolepis gracilipes invasion}

Frequently the earliest and worst effects of invasive ants are on the resident ant community (Holway et al. 2002). Thus, these communities, which are straightforward to sample, can provide an indication of the potential wider ecological effects of the invasive ant. It is often the case that the effects of invasive ants on the resident ant communities are density-dependent (e.g., Ross et al. 1996; Le Breton et al. 2005; Krushelnycky and Gillespie 2008). The ecological effects of A. gracilipes are clearly density-dependent on ant communities (Hill et al. 2003; Abbott et al. 2007; Lester et al. 2009; Hoffmann and Saul 2010; Drescher et al. 2011; Chapter 4) and other invertebrates (McNatty et al. 2009; Boland et al. 2011). It is also clear that not all populations of A. gracilipes are sufficiently abundant for negative ecological effects to occur (Abbott et al. 2007; Hoffmann and Saul 2010; Boland et al. 2011; Drescher et al. 2011), and that abundance also varies naturally over time (Lewis et al. 1976; Haines and Haines 1978b; Hill et al. 2003; Abbott 2006b; this study). The overall A. gracilipes abundance we measured at Nukunonu atoll appeared to be below a threshold at which negative ecological effects may be significant on the resident ant community. While this threshold is difficult to ascertain quantitatively, card count abundances may provide an approximate indicator. On Christmas Island in the Indian Ocean for example, management of $A$. gracilipes is initiated if card count values exceed 37, the point beyond which negative effects on native land crabs become apparent (Boland et al. 2011). With the exception of the new haplotype D invasions on Te Nonu 


\section{Chapter 5}

islet (card count 63) and Atafu atoll (maximum card count 63), our card counts did not exceed this value. By contrast, card count abundance measured on Christmas Island ranged between 14 and 136 in 2000 - 2002 (Abbott 2005), and card count abundance in the Northern Territory of Australia in 2009 ranged from 0 to 42 (Chapter 3). We advocate the use of card counts in A. gracilipes studies. Such an approach could enable a more accurate identification of the threshold at which density-dependent effects become apparent, enable comparison among studies, and highlight populations for which management options may be considered.

\subsubsection{Anoplolepis gracilipes population dynamics}

Although the differences in A. gracilipes abundance between 2004 and 2011 could be attributed to seasonal variation (Greenslade 1971; Baker 1976; Suwabe et al. 2009), we do not consider this to be the case. First, people on Tokelau were not concerned about the ant where it had persisted at low abundance since 2004. Residents were, however, concerned about the new A. gracilipes invasions, particularly on Atafu, where the ant had been noticeably abundant since 2008 . Second, we found no differences in ant community structure or species richness between A. gracilipes invaded and uninvaded sites on Nukunonu atoll in 2011. As ant communities may take time to assemble (Badano et al. 2005), they have likely adjusted to lower densities of A. gracilipes over some time. Finally, overall we found no significant difference in overall abundance between years the differences in abundance were primarily owing to the decline in haplotype D populations. 
Explanations for the decline in abundance of A. gracilipes haplotype D populations are not immediately obvious, as is often the case for population collapses of invasive species (Simberloff and Gibbons 2004). Decline of A. gracilipes on Mahé in the Seychelles was attributed to a reduction in protein resources (Haines and Haines 1978b), although a decline in protein availability appears unlikely on Tokelau. The decline in A. gracilipes abundance on inhabited islets was attributed by Tokelauan people to Cyclone Percy in 2005, prior to poisoning. Although we cannot be certain of the relative effects of the cyclone or of poisoning, new invasions have certainly occurred since the cyclone, and many of the existing populations appear to have persisted. The eagerness of Atafu residents for A. gracilipes to be controlled there provides an opportunity to undertake management, and determine its efficacy, focussing on areas where A. gracilipes is highly abundant.

Cycles of population explosion and decline appear to be a feature of a number of $A$. gracilipes invasions. Documented declines have occurred on the Seychelles (Lewis et al. 1976; Hill et al. 2003), and temporal variation has been noted on Christmas Island (Abbott 2006b). Similar declines have occurred in the Northern Territory of Australia (B. Hoffmann, personal communication). The causes of such declines among invasive species are not well understood, although disease is sometimes invoked (Simberloff and Gibbons 2004). A number of endogenous parasites are known to affect ant population dynamics, and have been explored as methods for biological control. For example, the red imported fire ant Solenopsis invicta has populations infected with a number of parasites including a microsporidian and viruses (Oi et al. 2008; Briano 2009; Valles et al. 2010). Anoplolepis gracilipes is known to harbour multiple endogenous bacteria (Sebastien et al. 2012; Chapter 6), although it is not yet known if these bacteria affect population dynamics. If parasites or disease are involved, their effects need not be catastrophic by themselves. Rather, they may decrease the population size to a point where Allee effects may result in compromised fitness (Allee 1931). The exploitation of Allee effects could be employed to more effectively manage invasions (Taylor and Hastings 2005; Pachepsky and Levine 2010; Tobin et al. 2011). 


\section{Chapter 5}

Although it is tempting to assign a pathogenic cause to population declines, invertebrate population dynamics often involve strong cycles of alternating high and low abundance (Lester and Burns 2008). In addition, the abundance of invasive ants and their effects on the recipient ant community may be higher shortly after arrival, and then decline to an equilibrium (Morrison 2002). Lester and Tavite (2004) and Abbott et al. (2007) inferred that the abundant A. gracilipes populations on the inhabited islets were newer arrivals, and we also observed the same pattern. The association that Abbott et al. (2007) found between A. gracilipes haplotype D abundance and effects on the resident ant community may thus have been owing to their recent arrival, rather than to the haplotype itself. The abundance and potential effects of A. gracilipes on the Tokelau atolls may therefore be higher in the period shortly after the initial invasion, although how long this period may be is unknown, as is any preceding lag time when the ants are not observed. By contrast, on Christmas Island, high abundance has been inferred to occur at a later stage in the invasion process (Abbott 2006b).

\subsection{Conclusion}

In Tokelau the correlation that Abbott et al. (2007) identified between haplotype D and greater abundance of $A$. gracilipes was no longer detected in 2011. Although overall the distribution of the ant has increased in Tokelau, many A. gracilipes populations have declined or disappeared on some islets. Understanding the population dynamics of invasions, particularly the underlying mechanisms promoting population decline, should be a high priority for invasion ecology (Taylor and Hastings 2005; Pachepsky and Levine 2010; Cooling et al. 2011; Tobin et al. 2011). Anoplolepis gracilipes has significant effects not only on ant communities and invertebrates, but also on forest birds (Davis et al. 2008; Davis et al. 2010), and arboreal- and ground-nesting seabirds (Feare 1999), which are common on Tokelau. Thus, we do not suggest that occasional population declines may eliminate the need for invasive species management, as considerable ecological damage may occur in the interim. Rather, understanding and exploiting the underlying mechanisms of decline of these populations could provide a useful aid to management. 


\section{Chapter 6. A preliminary metagenomic survey of Anoplolepis gracilipes endogenous parasite and symbiont communities}

\subsection{Abstract}

Invasive species often experience rapid increases in population size when introduced to a new range, which is followed by population stabilisation. However, occasionally declines of large populations of invasive species are observed, sometimes to the point of local extinction. Although the causes of these declines are difficult to ascertain, disease is often assumed. The invasive yellow crazy ant Anoplolepis gracilipes has been observed to experience unexplained population declines, and we hypothesised that these declines may be owing to endogenous bacterial or eukaryote parasite infection. We used Illumina GAIIx short-read sequencing of five samples, each of at least 50 ants that were pooled from A. gracilipes populations. Assemblies of the resulting reads were used to BLAST search existing public GenBank databases for potential eukaryote and prokaryote symbionts and parasites. Although our results suggested no evidence of eukaryotic parasites of insects, we found that bacterial community composition and diversity varied among our samples. Although the prevalence of many bacterial groups did not differ among A. gracilipes populations, the population with the lowest ant abundance had the lowest number of matches to bacterial sequences on the databases. Compared to other populations, this low abundance population also had a much higher prevalence of bacterial orders that have been identified as having beneficial relationships with ants, particularly involving antibiotic activity. The most prevalent of these orders was Burkholderiales, which has both pathogenic and beneficial members, but is only known to be beneficial in insects. This study has identified a range of bacteria that were previously unknown in A. gracilipes. Several of these are candidates for further research, which may shed light on the effects of endogenous parasites and symbionts on the population dynamics of the species. 


\section{Chapter 6}

\subsection{Introduction}

Invasive species often experience rapid increases in population size when introduced to a new range, which may be followed by population stabilisation to a lower level (Morrison 2002; Strayer et al. 2006). However, unexplained population declines of invasive species are occasionally observed, which sometimes lead to local extinction (e.g., Lewis et al. 1976; Morrison 2002; Hill et al. 2003; a number of studies reviewed by Simberloff and Gibbons 2004; Cooling et al. 2011). The causes of these declines are varied and difficult to determine, but may involve fluctuations in population dynamics, pathogens, or exhaustion of resources (Simberloff and Gibbons 2004). A number of parasites are known to affect insect population dynamics. For example, red imported fire ant (Solenopsis invicta) populations can be infected with a number of different parasites including a microsporidian and viruses (Oi et al. 2008; Briano 2009; Valles et al. 2010). Parasites not only affect the abundance of their hosts directly, but can also affect the host phenotype to ensure their own dispersal, to the detriment of the host (Yanoviak et al. 2008; Hoover et al. 2011).

The invasion success of pest species is sometimes attributed to a loss of natural enemies (herbivores, parasites or pathogens) in the invaded range (e.g., Mitchell and Power 2003; Torchin et al. 2003; Tsutsui et al. 2003a). However, when the host species is introduced to a new environment, a loss of parasites or symbionts from the native range may potentially be accompanied by exposure to a variety of new organisms that may be beneficial or harmful. The changes in the dynamics of these endogenous populations may cause changes in stress tolerance and parasite resistance, that could potentially affect invasion success (Feldhaar 2011).

The relationship between bacteria and their host insects is complex. Although endogenous bacteria may be harmful (Wenseleers et al. 2002), many are also beneficial (Dillon and Dillon 2003; Dale and Moran 2006; Feldhaar and Gross 2009), and if their function has a large effect on population dynamics, their loss could also result in population declines (Russell et al. 2009). For example, host sex-ratio manipulation in favour of females by bacteria such as Wolbachia, Arsenonophonus, and Rickettsia (Gherna et al. 1991; Engelstädter and Hurst 2009; Himler et al. 2011), may in some cases 
have positive effects on the fitness of the host by increasing abundance and spread (Himler et al. 2011). However, Wolbachia may reduce fitness among ants (Wenseleers et al. 2002), and a number of invasive ant species have exhibited a loss of Wolbachia in the introduced range (Shoemaker et al. 2000; Tsutsui et al. 2003a), which may contribute to their invasion success (Zientz et al. 2005). However, the function of common insect endosymbionts such as Wolbachia in ants is unclear and may vary (Zientz et al. 2005). Thus, the relationships between bacteria and their hosts can be complex and contextdependent.

Metagenomic studies may be a useful tool to assist in understanding the role of symbionts in the population dynamics of insects (Gosalbes et al. 2010). Studies of this nature can enable characterisation of endogenous communities, to generate and test hypotheses concerning interactions among members of these communities (Kunin et al. 2008). The advent of short-read (next / second generation) sequencing technologies enables metagenomic categorisation of entire communities, and the comparison of these communities among populations. Typically, metagenomic studies focus on bacterial communities, and target the small-subunit rRNA (16S rRNA) gene as it has a large reference database (Kunin et al. 2008). The two common approaches are using 454 pyrosequencing (Ishak et al. 2011; Tamaki et al. 2011), or Illumina short-read sequencing (Bartram et al. 2011; Caporaso et al. 2011). Eurkaryote parasites such as nematodes and fungi can also be detected using a metagenomics approach (Kunin et al. 2008), although studies of this nature are rare (Petrosino et al. 2009).

The yellow crazy ant, Anoplolepis gracilipes, is considered one of the most widespread and damaging invasive ants (Holway et al. 2002). Although it can occur at very high densities (O'Dowd et al. 1999; O'Dowd et al. 2003; Abbott 2006b), some populations of the ant are found at very low abundance, and unexplained population declines and disappearances have also been reported (Haines and Haines 1978b; Abbott 2006b; B. Hoffmann, personal communication; P. Green, personal communication; Chapter 5). Populations of A. gracilipes are known to harbour a number of endogenous bacteria, including Arsenophonus, Wolbachia, and Rhizobiales (Sebastien et al. 2012), although other bacteria and endogenous eukaryotes have not been investigated in the species. We hypothesised that there could be associations between the prevalence of a 


\section{Chapter 6}

wider variety of parasites and the variation in abundance of $A$. gracilipes. In our focal study population in Arnhem Land in the Northern Territory of Australia, the ants show patterns of abundance that differ spatially, and abundance also appears relatively low compared to a number of populations elsewhere (Chapters 4 and 5). Here we use a shortread sequencing metagenomic approach to detect candidate symbionts and parasites from a number of $A$. gracilipes populations. The aim of the study is to: 1) explore the endogenous metagenomic diversity and community composition of A. gracilipes; 2) identify differences in diversity and community composition in the Arnhem Land population. Our study is a preliminary analysis for the purpose of generating hypotheses for further exploration of the effects that these candidate endogenous organisms (and their interactions) may have on the population dynamics of $A$. gracilipes.

\subsection{Methods}

\subsubsection{Sample collection}

Samples were collected from Arnhem Land in Australia's Northern Territory (March 2009), Upolu in Samoa (October 2009), Christmas Island in the Indian Ocean (November 2009), and Okinawa in Japan (April 2010). Samples were collected from a single location in Upolu, Samoa, at three locations in Okinawa, Japan, and at a number of locations on Christmas Island and in Arnhem Land, Australia (Fig. 6.1). Individual ants were collected by aspirator while foraging or during nest excavations. Ants were stored in $95 \%$ ethanol at $4^{\circ} \mathrm{C}$, or in RNAlater (AMBION, Inc., Austin, Texas) at $-20^{\circ} \mathrm{C}$ and $-80^{\circ} \mathrm{C}$ (from six months after collection) until DNA extraction. All ants from Okinawa were stored in 95\% ethanol. Approximately half the samples from other sites were stored in ethanol, and the remainder in RNAlater.

Estimates of abundance were not obtained in Okinawa or Samoa, however, on Christmas Island and in Arnhem Land abundance estimates were primarily based on card counts at the site (see Green et al. 2004 for a description of the method), or visual assessment in some cases when ants were at very low abundance. The abundance of samples was categorised as high when card count values exceeded 30. Typically card count values for low abundance sites were zero. 


\subsubsection{Illumina GAIIx sequencing}

Ants from a number of collections were pooled into samples for sequencing in order to compare differences between populations (Fig 6.1). Ants were washed in ethanol to ensure no material remained on the integument. We extracted DNA from up to 10 ants at a time, using genomic kits (Bioline and Invitrogen), with further purification using a standard phenol and chloroform / isoamyl protocol, following which genomic DNA was precipitated in ethanol and dried. The DNA quantity was assessed using gel electrophoresis, and Nanodrop (Nanodrop Technologies Inc. 2005). The DNA extractions were of good quality, as indicated by gel bands at $\sim 10,000$ base pairs, minimal smearing and Nanodrop concentrations of $\sim 200+\mathrm{ng} / \mu \mathrm{l}$ and A260/280 ratios of $>1.8$, all of which met the standards expected for Illumina sequencing. The DNA from multiple extractions was pooled and was re-suspended in $20 \mu \mathrm{l}$ molecular grade $\mathrm{H}_{2} \mathrm{O}$ and precipitated in $44 \mu \mathrm{l}$ $100 \%$ ethanol and $2 \mu \mathrm{l} 3 \mathrm{M}$ sodium acetate for transport to the sequencing facility (Macrogen Inc. Seoul, Korea). Quality tests following library preparation at Macrogen met the standards for Illumina sequencing, which confirmed the results of our quality assessments.

The original design intention was to sequence populations as separate samples, and also separate high and low abundance populations from Christmas Island and Arnhem Land. Unfortunately, a number of samples were inadvertently incorrectly pooled by the sequencing facility (Table 6.1). Fortunately, one sample of Arnhem Land ants only at low abundance was preserved (Arnhem Land 4). A second sample had a high prevalence of ants from low abundance Arnhem Land sites (Arnhem Land 3). Libraries were created for each of the five samples and each sample was run on a single lane of an Illumina Genome Analyzer IIx (GAIIx; five lanes in total) producing paired-end reads of 101 bases. Postprocessing at Macrogen included an analysis pipeline using RTA v 1.8 and CASAVA v 1.7.0 (Illumina). Sequencing resulted in an average of 7.7 Gbases of data per lane and a total of 34.5 Gbases over the five lanes. 


\section{Chapter 6}

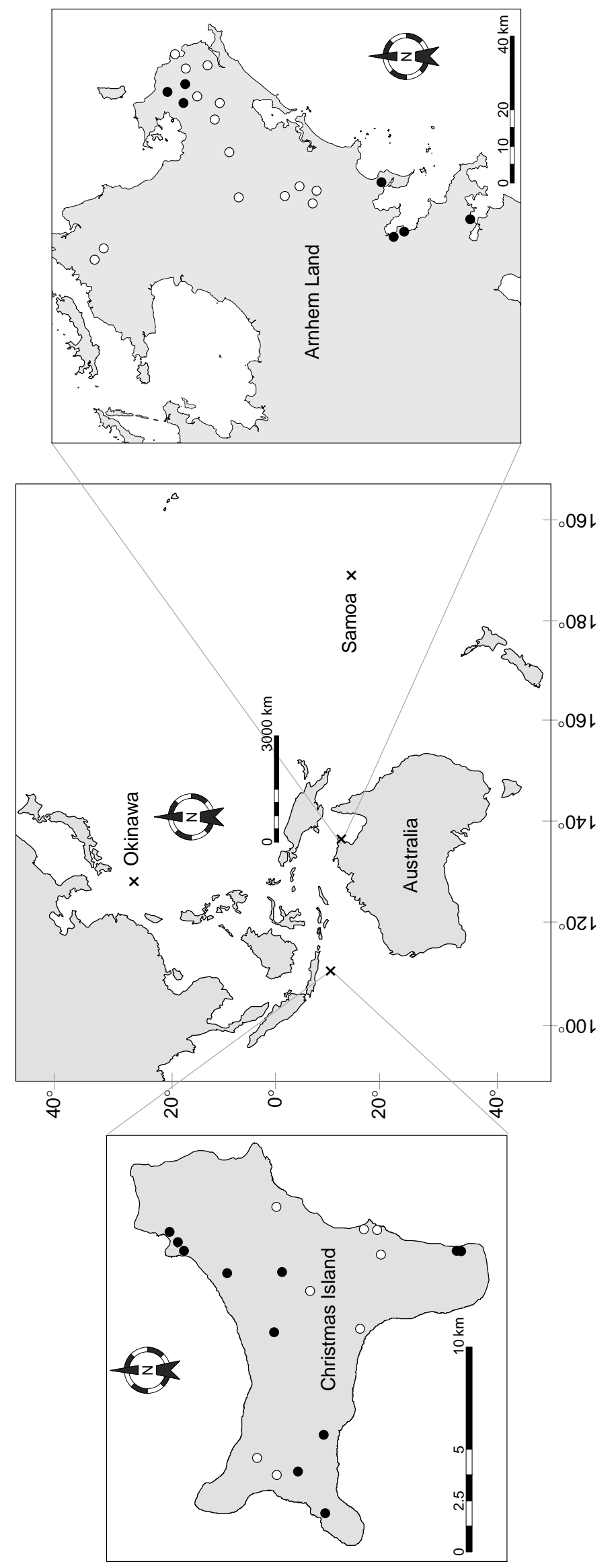

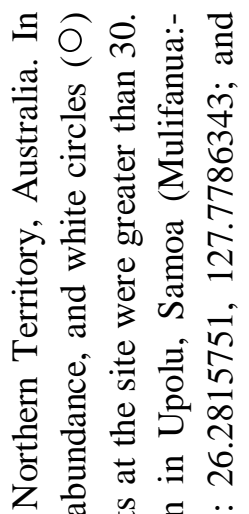

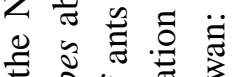

: : :

चี के

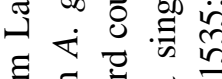

ฮี

安至造

击

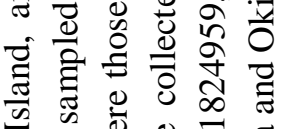

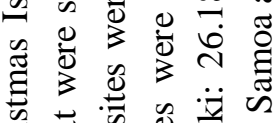

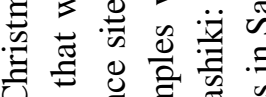

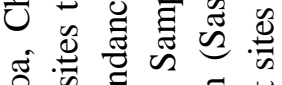

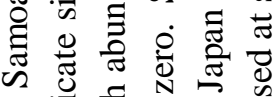

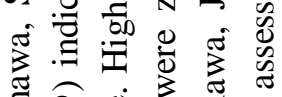

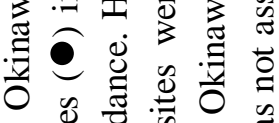

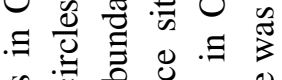

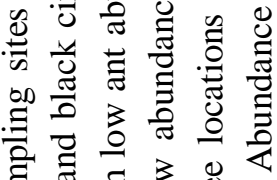

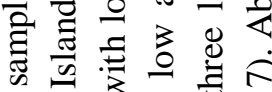

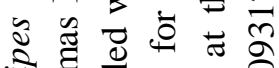

इ

क⿺辶寸

$\approx$ б

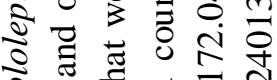

ई

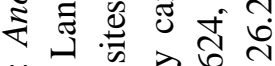

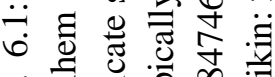

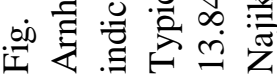




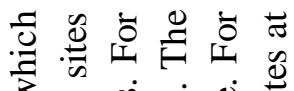

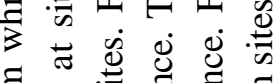

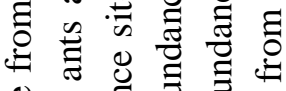

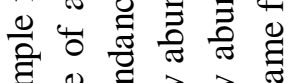

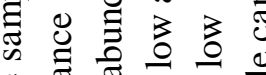

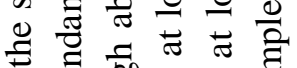

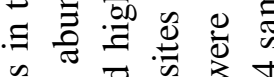

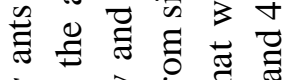

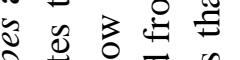
: :

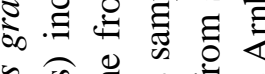

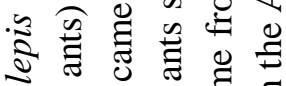

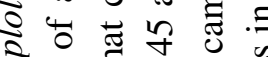

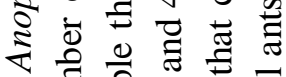

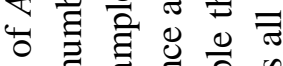
¿ छ है.气

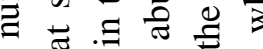

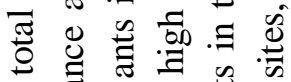

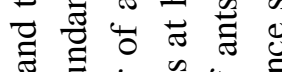

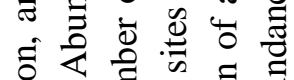

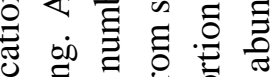
을

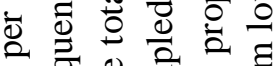

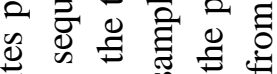

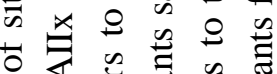
एँ

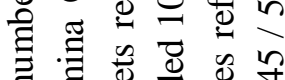
过焉至脑

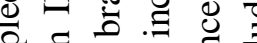

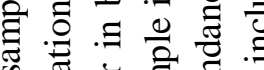

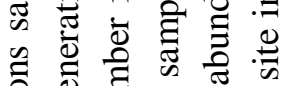
.

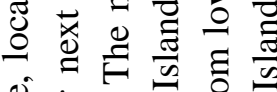

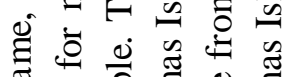
‡

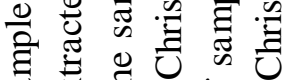
矛芯导

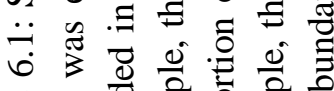

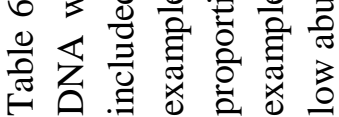

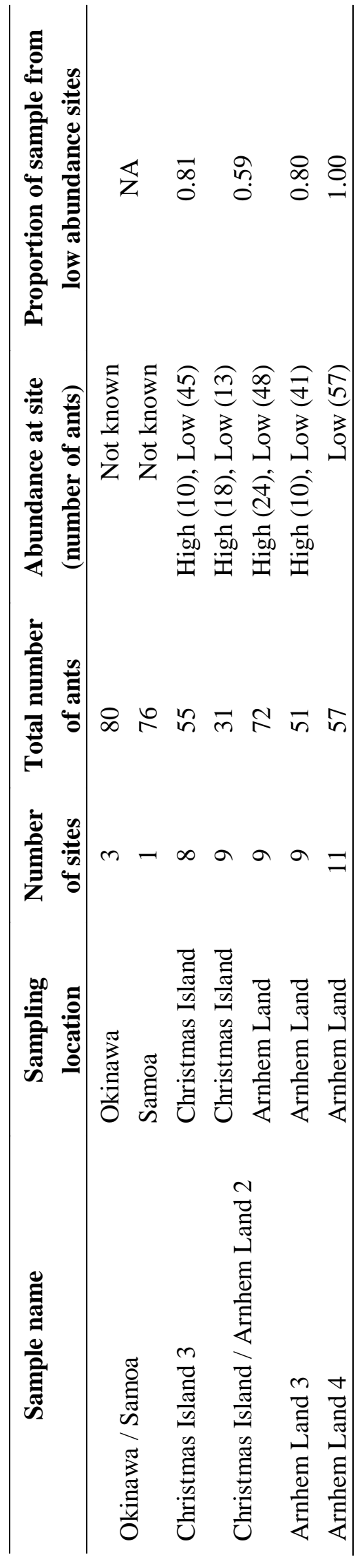




\section{Chapter 6}

\subsubsection{Quality assessment of Illumina GAIIx sequencing}

To validate the quality of the sequencing run we used the custom written java package SolexaStats (see Appendices). This function calculates the proportion of bases in the sequence files where the FASTQ $Q$ value (quality score) exceeded 30, which is equivalent to a base call accuracy of $99.9 \%$ (i.e., the probability of a base being incorrect is 0.001 ). SolexaStats revealed an average $Q$ value $>30$ of 0.91 (Table 6.2), which exceeded the minimum guidelines recommended by Illumina (Illumina 2011), which suggest that $>0.80$ of $Q$ values should be greater than 30 .

To assess the quality of the run and detect systemic errors in the data we used SolexaQA.pl v 1.7 (Cox et al. 2010), using the software default of $P=0.05(Q<13$, or 1 base call error every 20 nucleotides). SolexaQA analysis found occasional poor quality scores for sequences in the first two lanes of data. These had little effect on the overall quality of the run, and were not apparent in later lanes. We then used DynamicTrim.pl v 1.7 (Cox et al. 2010) to exclude bases with a more stringent $Q<30$ or $P=0.001$ (1-in1000 probability of an incorrectly called base). We used this stringent quality score in order to minimise the inclusion of poor quality sequences. Trimming with DynamicTrim.pl to retain bases with scores of $Q>30$ reduced file sizes to $80.4 \%$ of the original size, and average sequence length to 74 bases (Table 6.2). The LengthSort.pl v 1.7 (Cox et al. 2010) script was then used to remove sequences that were less than 25 base pairs in length. Once sequences shorter than 25 base pairs were removed, the average file size was $76.8 \%$ of the original size. In some cases, one of the paired-end reads (usually the second) was shorter than the 25 base pair threshold. This is typical of GAIIx data, where the second read of the pair is often less well resolved. LengthSort.pl separated these into single-end read file, which included $4.5 \%$ of the data on average, or approximately 3 million reads per file (Table 6.2). 


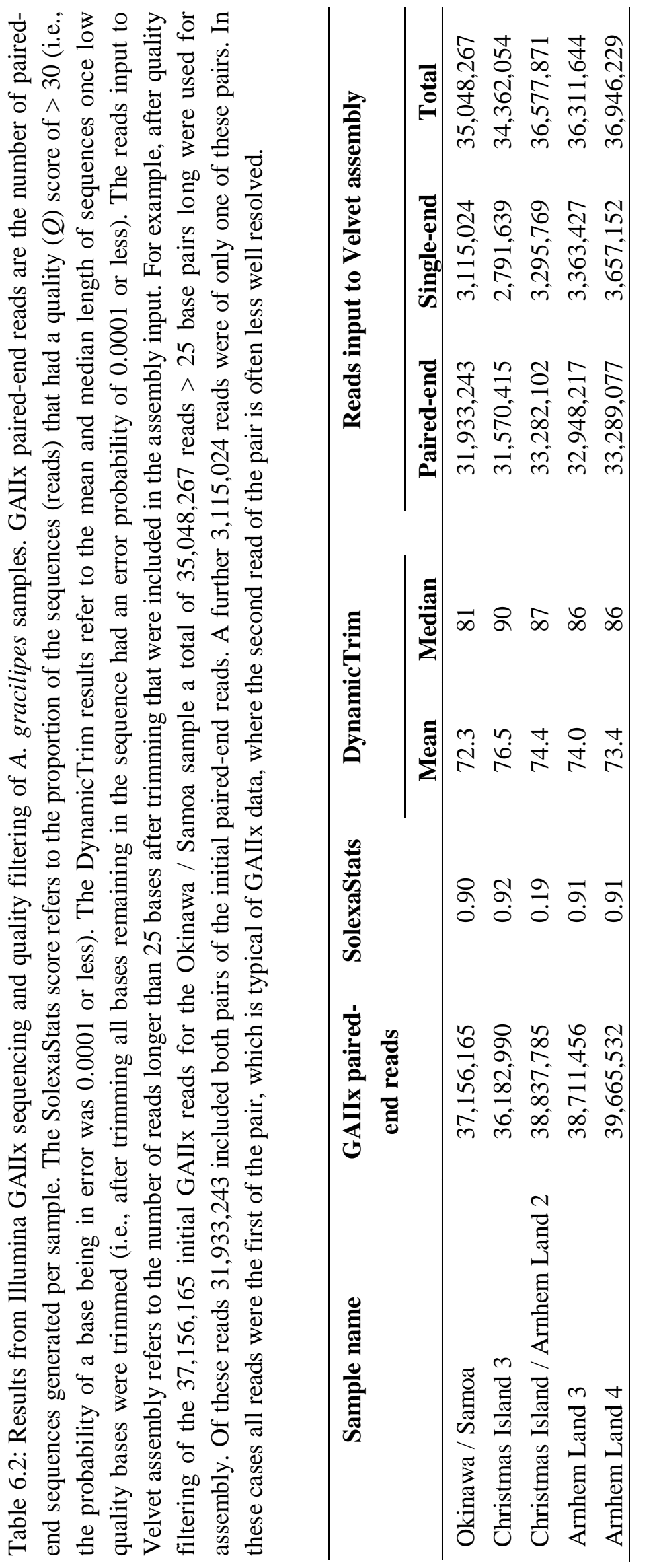




\section{Chapter 6}

We also used FastQC v 0.9.6 (Andrews 2011) to determine other indicators of poor quality including over-represented sequences, and inequality in base composition. FastQC analysis revealed that sequences in the second of the pair of reads were sometimes overrepresented by unresolved repeat sequences (NNNNn). These made up on average only $0.30 \%$ of all bases (compared to $0.16 \%$ of bases in the first of the pair of reads). These repeats are generally caused by sections which were joined by read-pairs but separated by a repeat pattern that could not be resolved (Andrews 2011). We trimmed these unresolved repeats using LengthSort.pl. FastQC identified a base inequality in all lanes, which consisted of an $\mathrm{A}+\mathrm{T}$ bias $(\mathrm{A}+\mathrm{T}=0.68)$. All ant genomes sequenced have shown this feature (Bonasio et al. 2010; Smith et al. 2011a; Smith et al. 2011b; Suen et al. 2011; Wurm et al. 2011), thus we consider the bias was not owing to any failure in sequencing. FastQC identified a number of 5-mers that appeared in $3-5$ fold greater number than expected. These consistently involved GC rich sequences such as CGCGC, GCGCT, CCGCG etc. As FastQC calculates the expected level at which a given 5-mer should have been observed in the sequence based on the base content of the library as a whole, the AT bias in our dataset may have caused an over-representation of GC / CG related patterns in this analysis.

\subsubsection{Genomic sequence assembly}

We assembled the genomic sequences using Velvet $\mathrm{v}$ 1.1.0.5 (Zerbino and Birney 2008; Zerbino 2010). Subsequent to quality checking and file trimming, paired-end sequence files were interleaved using the shuffleSequences_fastq.pl Perl script bundled with Velvet. Both the shuffled paired-end files and the single-end files output from LengthSort.pl were used in the assemblies. The VevetOptimiser.pl Perl script (Gladman 2011) was used to determine optimal assembly parameters. The initial parameter settings to determine the optimum parameters used k-mer lengths of between 43 and 57 . We calculated the mean coverage for contigs > 100 base pairs in each lane in $\mathrm{R} v 2.14 .0$ (Ihaka and Gentleman 1996; R Development Core Team 2011). Our Velvet assemblies resulted in between $331 \mathrm{k}$ and 440k contigs per lane (Table 6.3). N50 values (50\% of the assembly in contigs equal to or longer than this value) ranged from 1,656 to 4,052 bases (Table 6.3). Coverage, contig size, N50 and total bases in contigs were negatively related to k-mer length, which is typical of velvet assemblies. Longer k-mer length was also 
associated with many more unused reads, with up to $55 \%$ of input reads not used in assemblies. However, longer k-mer lengths resulted in more contigs longer than 1,000 bases (Table 6.3).

\subsubsection{BLAST sequence similarity searching and post-processing}

To detect potential parasite and symbiont sequences in the assembled data, we used BLAST searches to query the assemblies against publicly available genome data. We used the BLASTn algorithm in BLAST+ to search the NCBI microbial 16S ribosomal sequence (16SMicrobial), non-model organism whole genome sequence (wgs), nonmodel organism genomic (other_genomic), and nucleotide (nt) databases. The databases downloaded from GenBank (ftp://ftp.ncbi.nlm.nih.gov/blast/db/) were dated 5 December 2011. Searches were run locally on the Victoria University of Wellington (VUW) high performance computing facility on which the BLAST+ executables were installed (BLAST v 2.2.25; Zhang et al. 2000).We restricted searches to a threshold e-value of 0.0001 or lower, and used a word size of 11 base pairs, to enhance the specificity of the searches (Madden 2002). In practice the e-values in this study were orders of magnitude lower than the threshold, which indicated high-scoring, relatively specific results.

Once the BLAST searches had completed we used a custom Perl script taxon4blast.pl (see Appendices) to summarise the data. The taxon4blast.pl script accepts as input up to $n$ files in BLAST+ output format 6 , and has sub-routines to: 1) append taxonomic information to individual BLAST results from local copies of the NCBI taxonomy database (ftp://ftp.ncbi.nih.gov/pub/taxonomy); 2) summarise BLAST results by a user specified taxon level (genus, subfamily, family, order, class, phylum, kingdom or superkingdom,); 3) summarise the shared and unique results among the input files, also by user specified taxon level; 4) summarise the shared and unique results among the input files, where only exact sequence matches are included; 5) extract the original sequences input to BLAST for a specified taxon level (either to retain or discard those sequences from the original input file). We summarised our results by genus, family and order for community composition analyses, and report detailed results by genus and order. 


\section{Chapter 6}

Ð

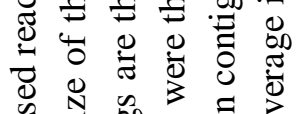

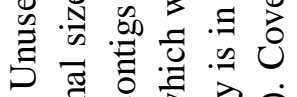

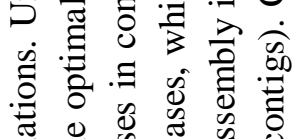

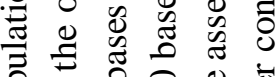

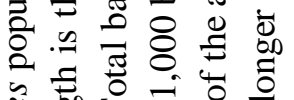

के

홍 छ

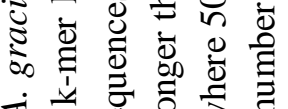

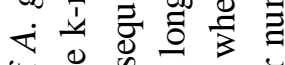

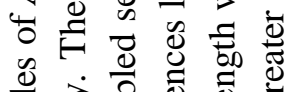

嵌

है छै

च

\&

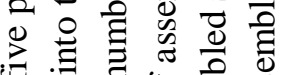

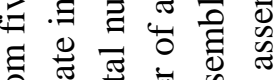

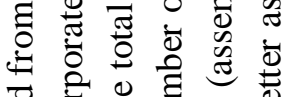

论

政.

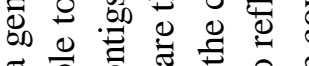

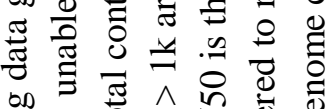

of

产

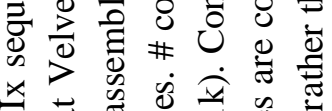

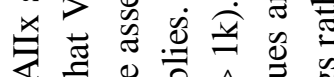

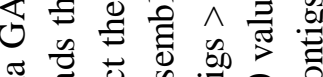

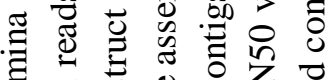

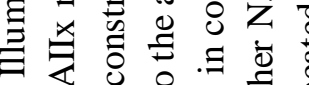

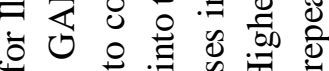

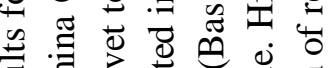

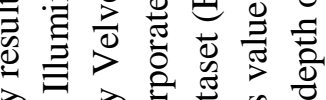

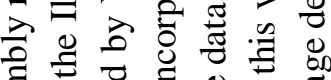

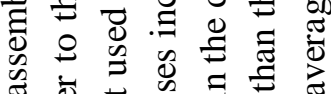

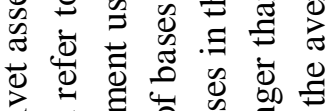

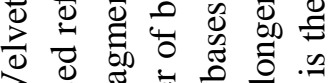

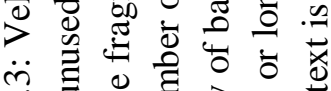

ชั

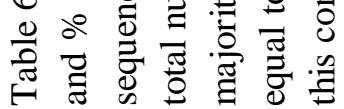

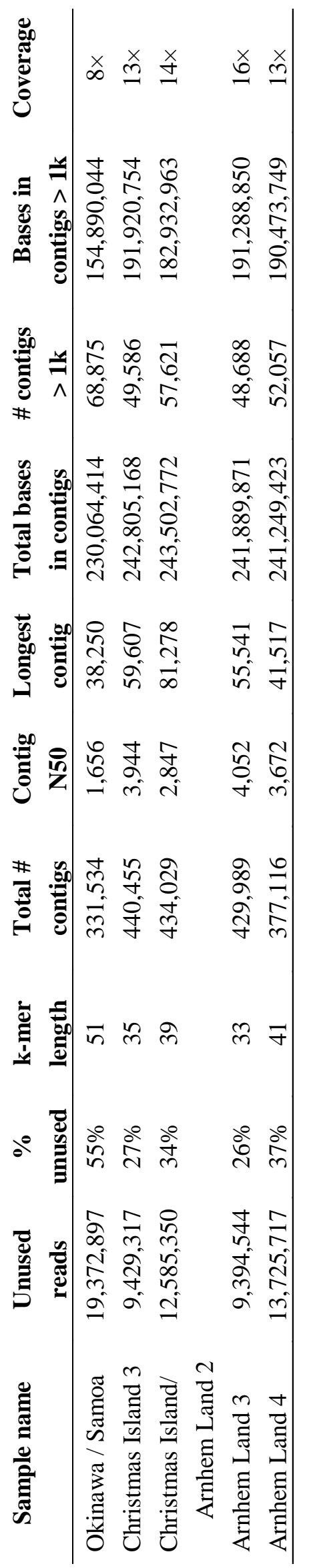


Similarity in BLAST search results is not a guarantee of identity, and results are dependent on the sequences of taxa lodged in the databases. In order to further exclude possible spurious matches, we used a number of criteria to prioritise the likely accuracy of the summarised BLAST matches. At the genus level, with the exception of the 16SMicrobial database we considered that matches on ant, Hymenopteran and other insect taxa were most likely to be homologues. Results of this nature were orders of magnitude more prevalent than matches for other taxa. Thus, we excluded all BLAST matches on eukaryote sequences that had low scores and were thus highly unlikely to have been biologically valid as potential parasites or symbionts. Results such as this we considered to be owing to the large number of eukaryote sequences present on the databases, and highly conserved sequence similarity, possibly along with undigested gut contents of the ants. The results for genera were thus limited to those with a relatively high numbers of matches, longer total lengths (these two measures were typically correlated), or longer average lengths than the potentially artefactual matches. This may have resulted in a subset result dataset that excluded valid results, but should have provided a conservative survey that did not inflate the prevalence of potential parasites.

\subsubsection{Community composition and diversity}

To determine whether metagenome community structure differed among our sampled populations, we used non-metric Multi-Dimensional Scaling (MDS), ANOSIM and SIMPER implemented in PRIMER v 6.1 .11 (Clarke and Warwick 2001; Clarke and Gorley 2006). We used number of matches as the measure of abundance, log-transformed to even out the effects of rare and abundant taxonomic groups, and used the Bray-Curtis index of similarity measure as recommended by Clarke and Warwick (2001). The MDS was run using 1000 iterations with a Kruskal stress formula 1 and a minimum stress of 0.01. We used ANOSIM analyses run over 9999 permutations for significance testing of the differences between the Arnhem Land samples and other samples. In ANOSIM analyses the Global $R$ statistic ranges from -1 to 1 (but typically from 0 to 1 ). Random grouping of samples is indicated by $R$ values close to 0 , and as $R$ approaches 1 replicates within a group are increasingly more similar to each other than to replicates within other groups (Warwick et al. 1990). As the sample size was small we considered the ANOSIM analysis indicative only of the degree of difference between groups (i.e., the size of the 


\section{Chapter 6}

Global $R$ statistic) rather than statistical significance of the result. Separate analyses were conducted by order, family, and genus for all databases. As potential eukaryote parasites did not figure strongly (see Results section 6.4.1) we limited these analyses to bacterial groups in all the databases.

\subsection{Results}

\subsubsection{BLAST sequence similarity}

At the genus level the highest number, and longest total length of matches (i.e., closest matches) were to other ant genera, other Hymenopterans, and insects in the wgs and other_genomic databases (e.g., Camponotus, Linepithema, Solenopsis, Harpegnathos, Acromyrmex, Atta, and Pogonomyrmex in the wgs database, in that order, followed by Bombus and Apis). The next most common matches were to other insects or to bacteria (often Escherichia and Wolbachia). Ants alone made up 85 - 90\% of matches in each sample in the wgs database. In the other_genomic database matches to ant sequences were lower than in the wgs database ( $70-76 \%$ of each sample), because sequences for the ant genera Camponotus, Harpegnathos and Pogonomyrmex were not lodged in the other_genomic database. Matches to ant sequences within the nt database were very low, in contrast to the comparatively large amount of ant genomic data in the wgs and other_genomic databases, also because the database contained less data for these groups than other groups.

No sequences of fungi, nematode or other potential eukaryote parasites were found among the most common matches in the wgs, other_genomic and nt database results. Matches to bacterial sequences were at high prevalence in all databases. Thus, the remainder of our results focus on the bacterial sequence matches in all databases. The number and lengths of matches for each genus reflected the sizes of the databases (Table 6.4). Many more sequence matches were obtained from the larger wgs, nt and other_genomic databases than from the smallest 16SMicrobial database (Table 6.4). 
In all databases the search results for the Arnhem Land 4 sample differed substantially from the other samples. Compared to other samples, this sample had fewer matches to sequences in the wgs, other_genomic and nt databases, and the total length of all matches was shorter in all the databases (Table 6.4). The number of genera found did not differ substantially among the samples for any of the databases, although the Arnhem Land 4 sample had the highest number of matches to genera in the 16SMicrobial database (Table $6.4)$.

\subsubsection{Community composition and diversity}

The bacterial community composition of sequence matches among A. gracilipes samples showed a number of similar patterns. Across all the databases and levels of taxonomic hierarchy, the Arnhem Land 4 sample of ants from low abundance sites tended to be somewhat differentiated from other samples (Fig. 6.2). This was particularly evident in the nt database (Fig. 6.2, j-1), and in the 16SMicrobial database at the family level (Fig. 6.2, b). The Arnhem Land 3 sample, which included a mixture of ants from low and high abundance sites tended to group with the other samples (e.g., Fig. 6.2, k), or was intermediate between the Arnhem Land 4 sample and other samples (e.g., Fig 6.2, c).

In some cases the ANOSIM analyses found differences in community composition among the samples (Fig. 6.2). Analyses of the 16SMicrobial database results suggested the Arnhem Land samples were distinct from the other samples (ANOSIM, order: Global $R=0.63$; family: Global $R=0.58$; genus Global $R=0.67$ ). The community composition of the Arnhem Land samples in the wgs database results were more distinct from the other samples at the genus level (Global $R=0.33$ ), but less so at the order level (Global $R$ $=0.17$ ), and not at all at the family level (Global $R=0.00$ ). In the other_genomic database results the community composition of the Arnhem Land samples was not distinct from the other samples (ANOSIM, order: Global $R=-0.17$; family: Global $R=$ 0.08; genus Global $R=0.17$ ). ANOSIM analyses in the nt database results identified the community composition of the Arnhem Land samples as distinct from the other samples at the order (Global $R=0.50$ ) and genus levels (Global $R=0.58$ ), but not at the family level (Global $R=0.08)$. 


\section{Chapter 6}
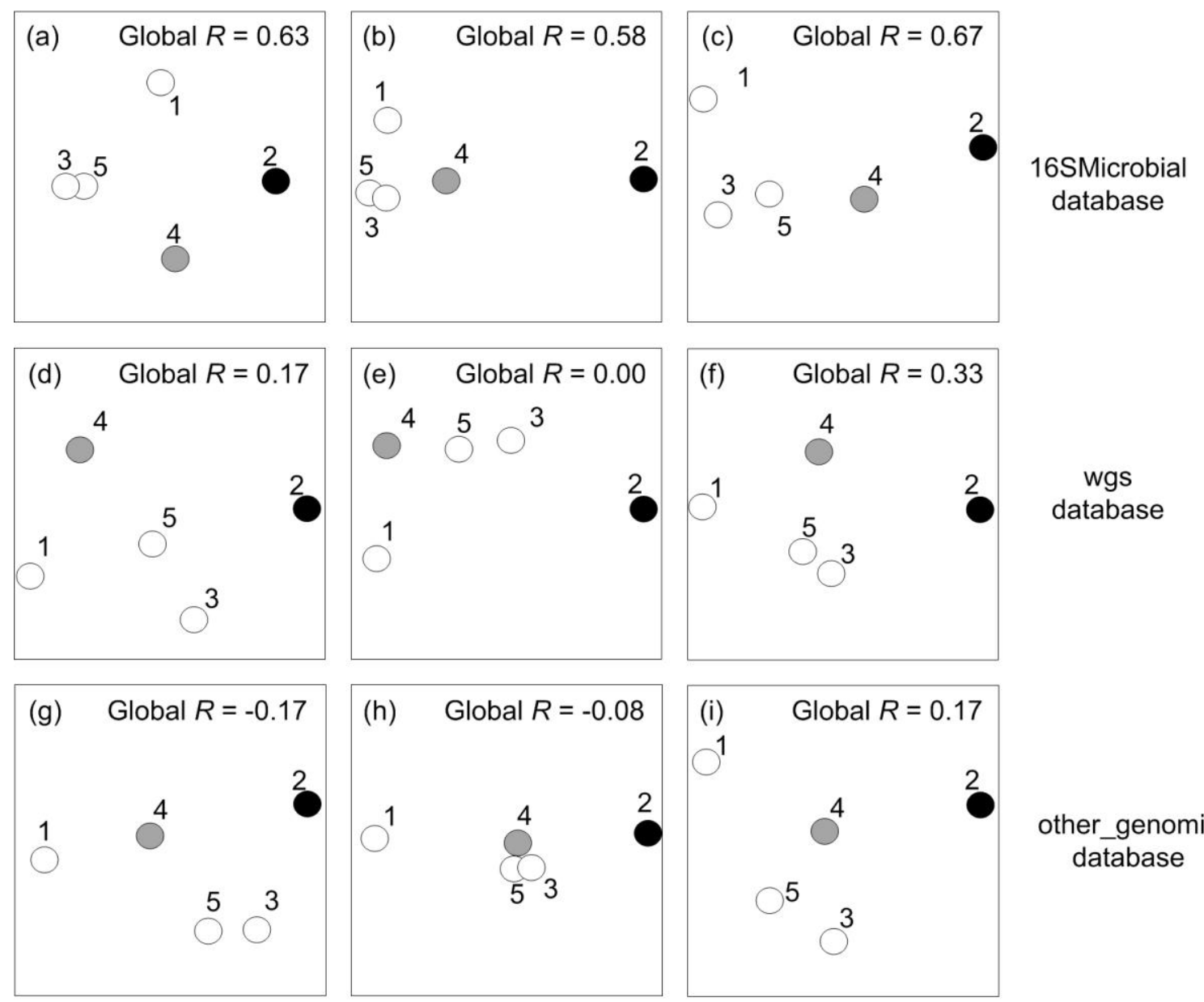

other_genomic database

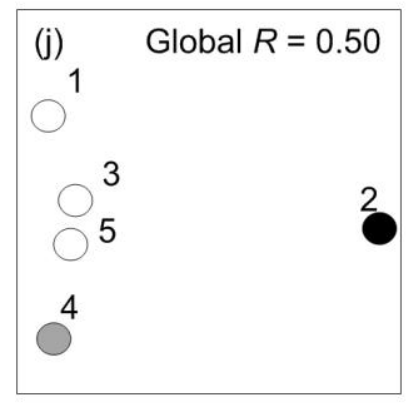

Order

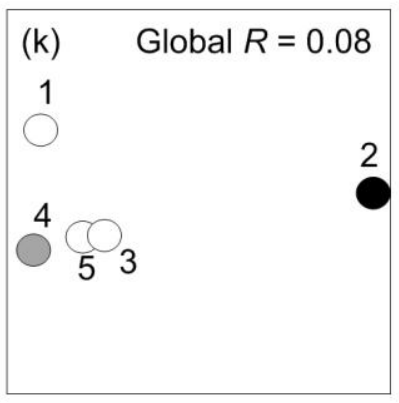

Family

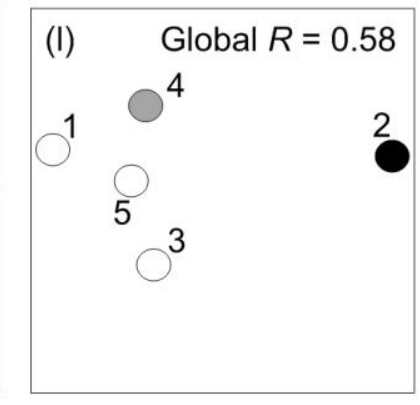

Genus

nt database

Fig. 6.2: Two-dimensional MDS plots of differences in A. gracilipes endogenous bacterial community composition based on BLAST search results. The MDS was based on BrayCurtis similarity of $\log (\mathrm{X}+1)$ transformed data, analysed by order, family and genus: $(\mathrm{a}-\mathrm{c})$ 16SMicrobial database; $(\mathrm{d}$ - f) other_genomic database; (g - i) wgs database; $(\mathrm{j}-1) \mathrm{nt}$ database. Filled circles indicate the Arnhem Land samples (black $=$ Arnhem Land 4; grey $=$ Arnhem Land 3) and open circles indicate other samples. The numbers refer to the sampling sites: $1=$ Okinawa $/$ Samoa; $2=$ Arnhem Land 4; $3=$ Christmas Island $/$ Arnhem Land 2; $4=$ Arnhem Land 3; 5 = Christmas Island 3. 


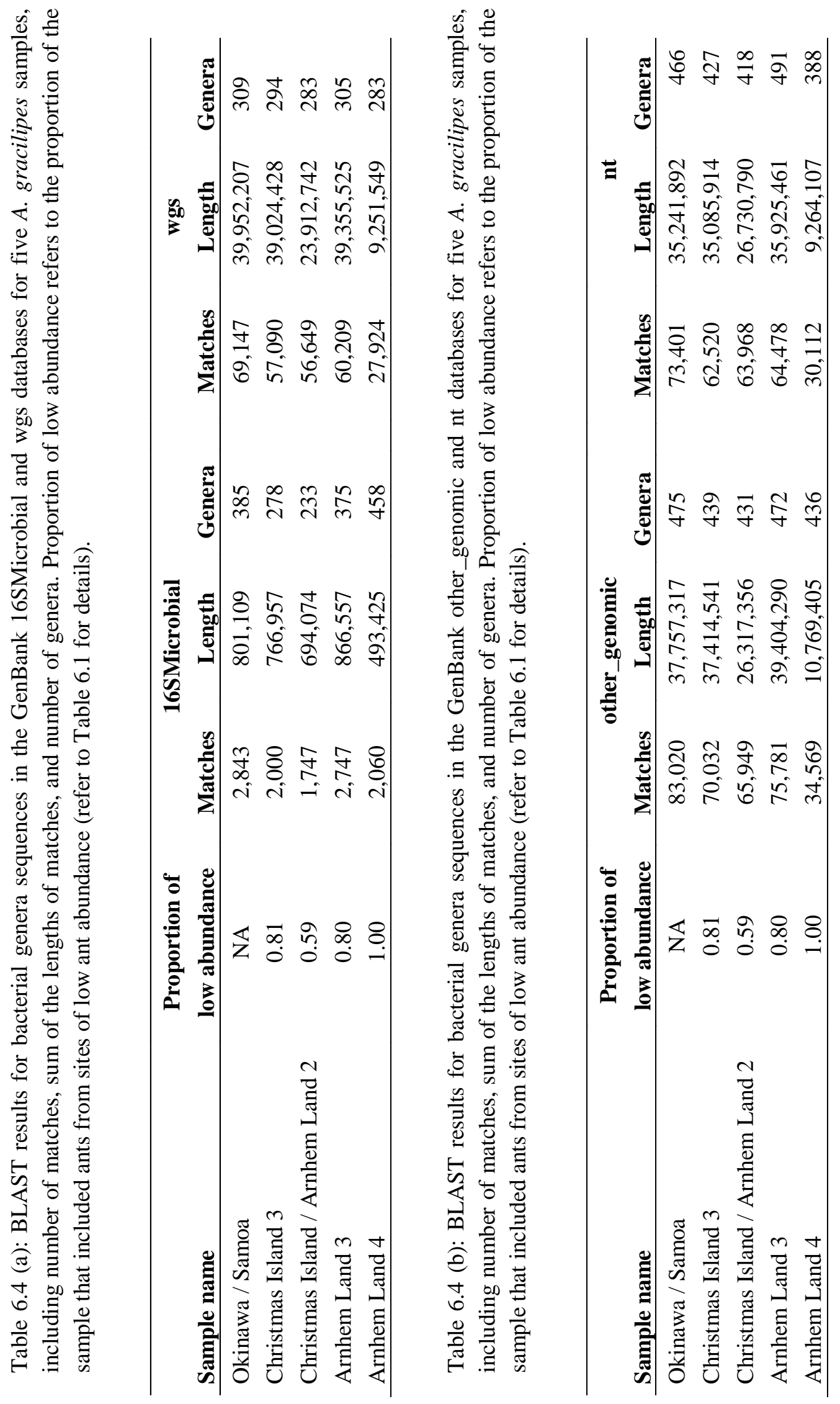




\section{Chapter 6}

\subsubsection{GenBank 16SMicrobial database composition}

At the order level in the 16SMicrobial database results, many bacterial groups were common to all samples, although there were some notable differences in the Arnhem Land 3 and 4 samples. The most common matches in all samples were to the orders Rhizobiales, Enterobacteriales, Vibrionales, Rhodobacterales, Rickettsiales, Pseudomonadales, Rhodospirillales, Rhodocyclales, Alteromonadales, and Sphingomonadales (Fig. 6.3) to varying degrees. Members of the Burkholderiales and Actinomycetales orders were relatively more common in the Arnhem Land samples, and rare in other samples. Matches to Enterobacteriales were less common in the Arnhem Land samples (Fig. 6.3), as were the Alteromonadales in the Arnhem Land 4 sample. Matches to Rhodospirillales featured highly in the Okinawa / Samoa sample.

Consistent with the results at the order level, at the genus level Burkholderia (order Burkholderiales) was the most commonly matched genus in the Arnhem Land samples, while Vibrio (order Vibrionales, Fig. 6.3), and a variety of genera in the Enterobacteriales order were common among all samples. Acetobacter (order Rhodospirillales, Fig. 6.3) was the most commonly matched genus in the Okinawa / Samoa sample, but was rare or absent in other samples. 


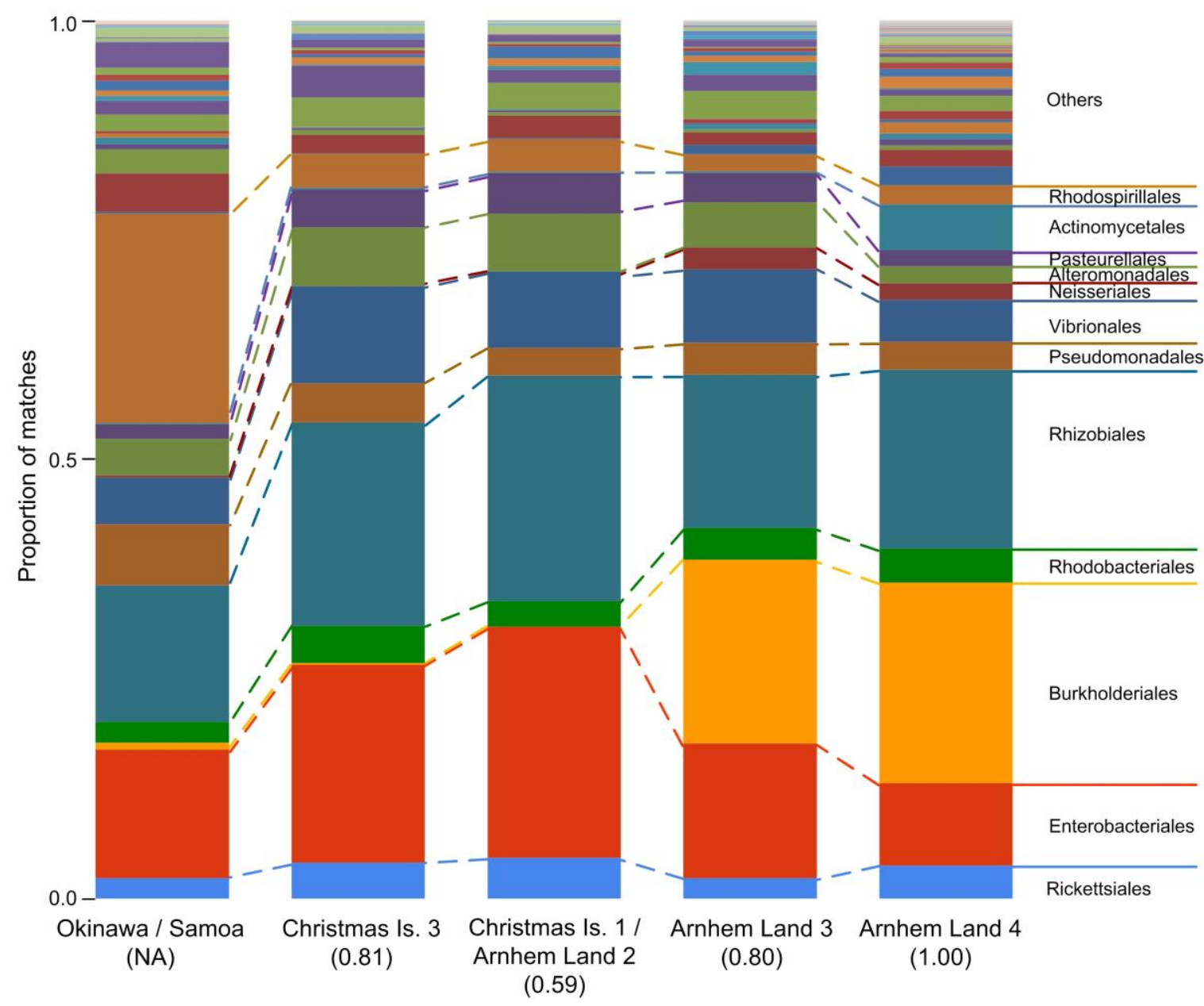

Fig. 6.3: Summary of BLAST matches to bacterial orders in the 16SMicrobial database for five A. gracilipes samples. The figure in brackets below the sample name is the proportion of the sample that included ants from sites of low abundance (refer to Table 6.1 for details). 


\section{Chapter 6}

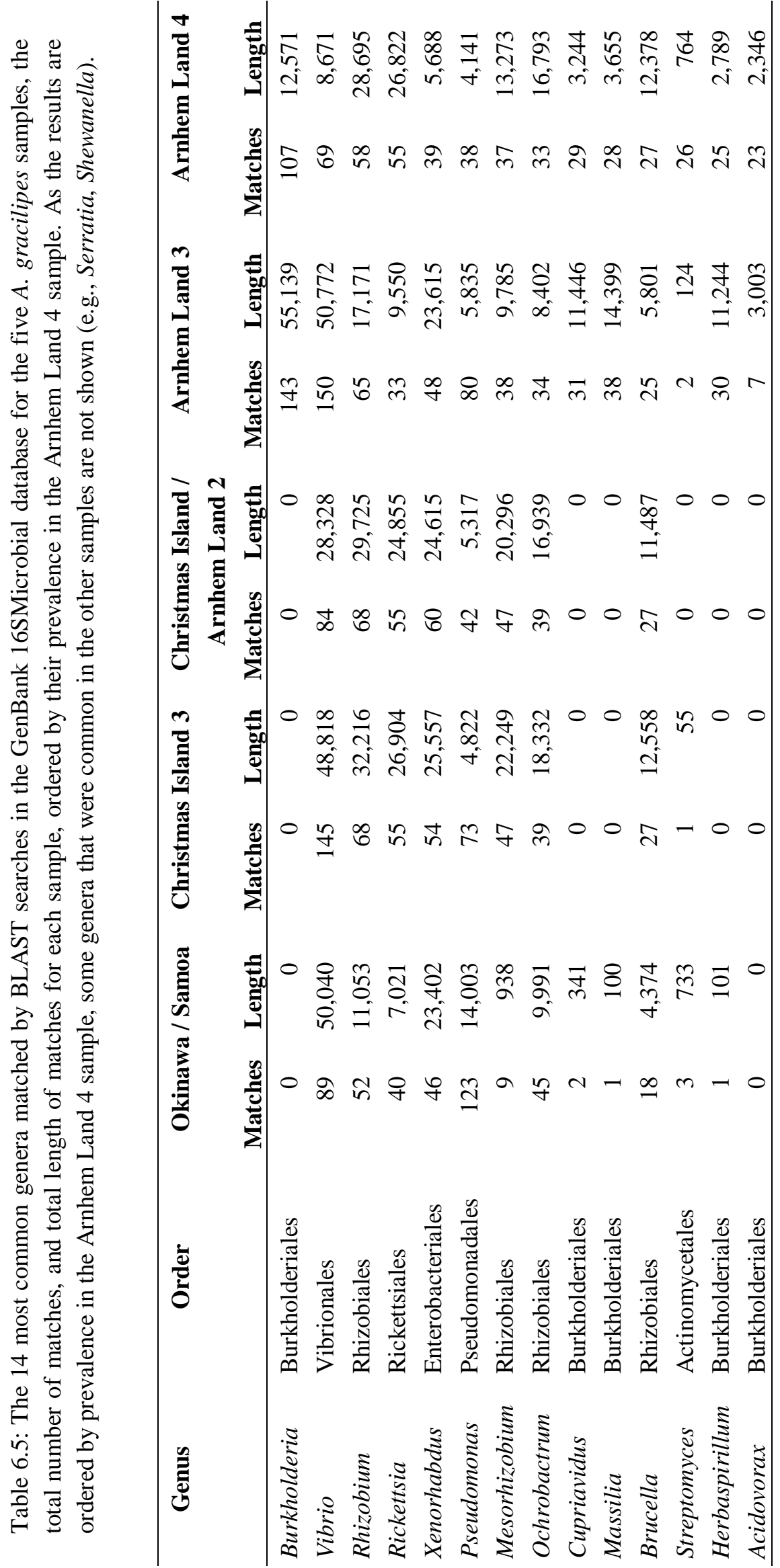




\subsubsection{GenBank wgs database composition}

The pattern of matches to orders in the wgs database was similar to the 16SMicrobial database. The most remarkable result in the wgs database was a very low number of matches to bacterial sequences in the Arnhem Land 4 sample compared to the other samples (Table 6.4), with the exception of Wolbachia (Table 6.6). Matches to Escherichia sequences were the most prevalent in all samples except Arnhem Land 4, where the number of matches was relatively low (Table 6.7). The genus Burkholderia (order Burkholderiales) was of interest as it was prevalent in the 16SMicrobial results for the Arnhem Land samples. Sequences of this genus received matches for all samples, and, although much less common than Wolbachia (order Rickettsiales), matches to it were the second most prevalent bacterial genus and order in the Arnhem Land samples (Table 6.6; Fig. 6.4). The related Rickettsia (order Rickettsiales) received matches in all samples, but with relatively low prevalence. Other bacterial genera (e.g., Salmonella, Vibrio and Yersinia) that received matches in all samples were at very low prevalence in the Arnhem Land 4 sample. 


\section{Chapter 6}

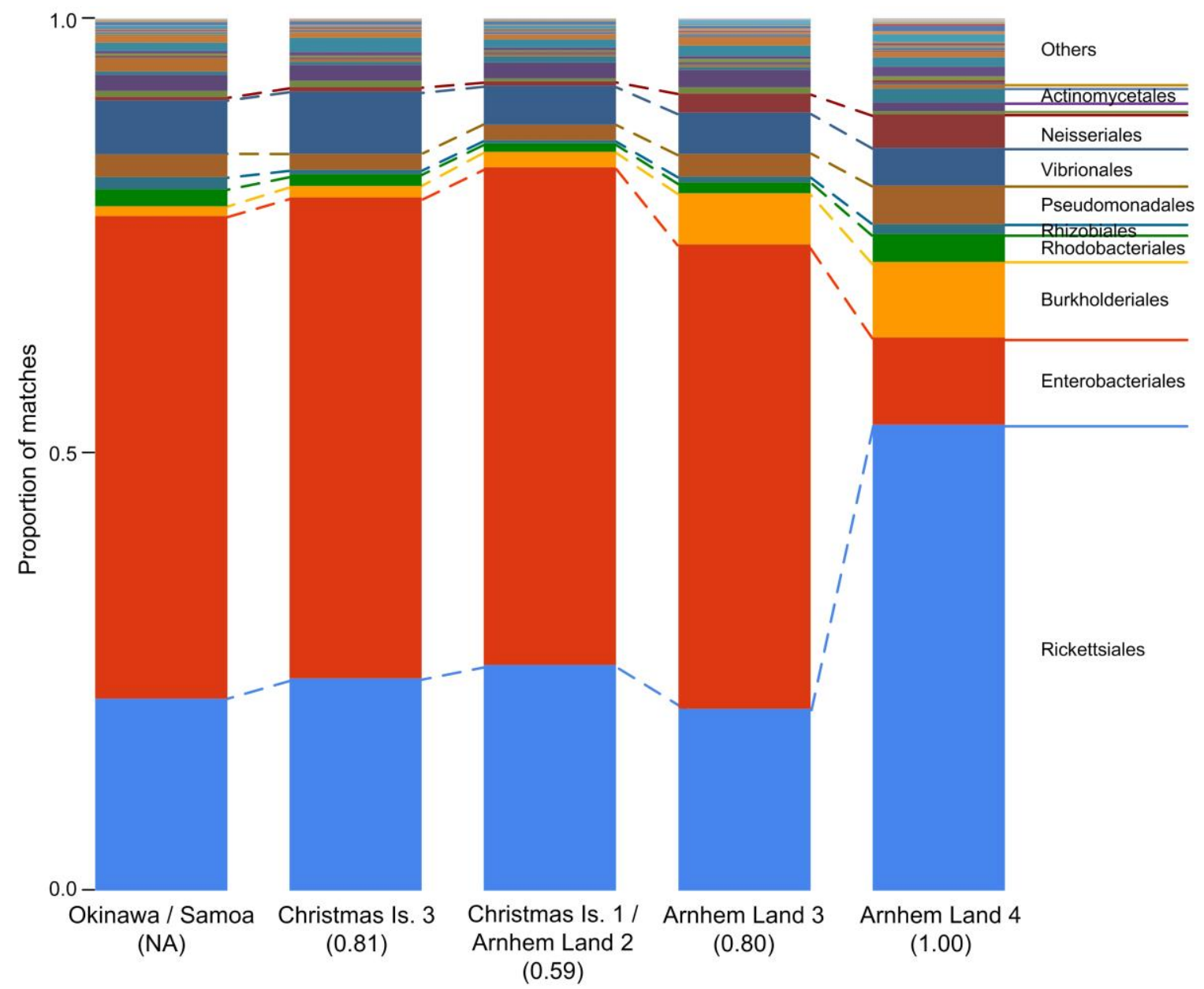

Fig. 6.4: Summary of BLAST matches to bacterial orders in the wgs database for five $A$. gracilipes samples. The figure in brackets below the sample name is the proportion of the sample that included ants from sites of low abundance (refer to Table 6.1 for details). The apparently high prevalence of matches to Rickettsiales (primarily sequences belonging to the genus Wolbachia) in the Arnhem Land 4 sample is open to misinterpretation. Matches to Rickettsiales sequences were no more prevalent than other groups in this sample (Table 6.7), and the distortion is owing to the markedly low number of matches to all other orders in the Arnhem Land 4 sample. 


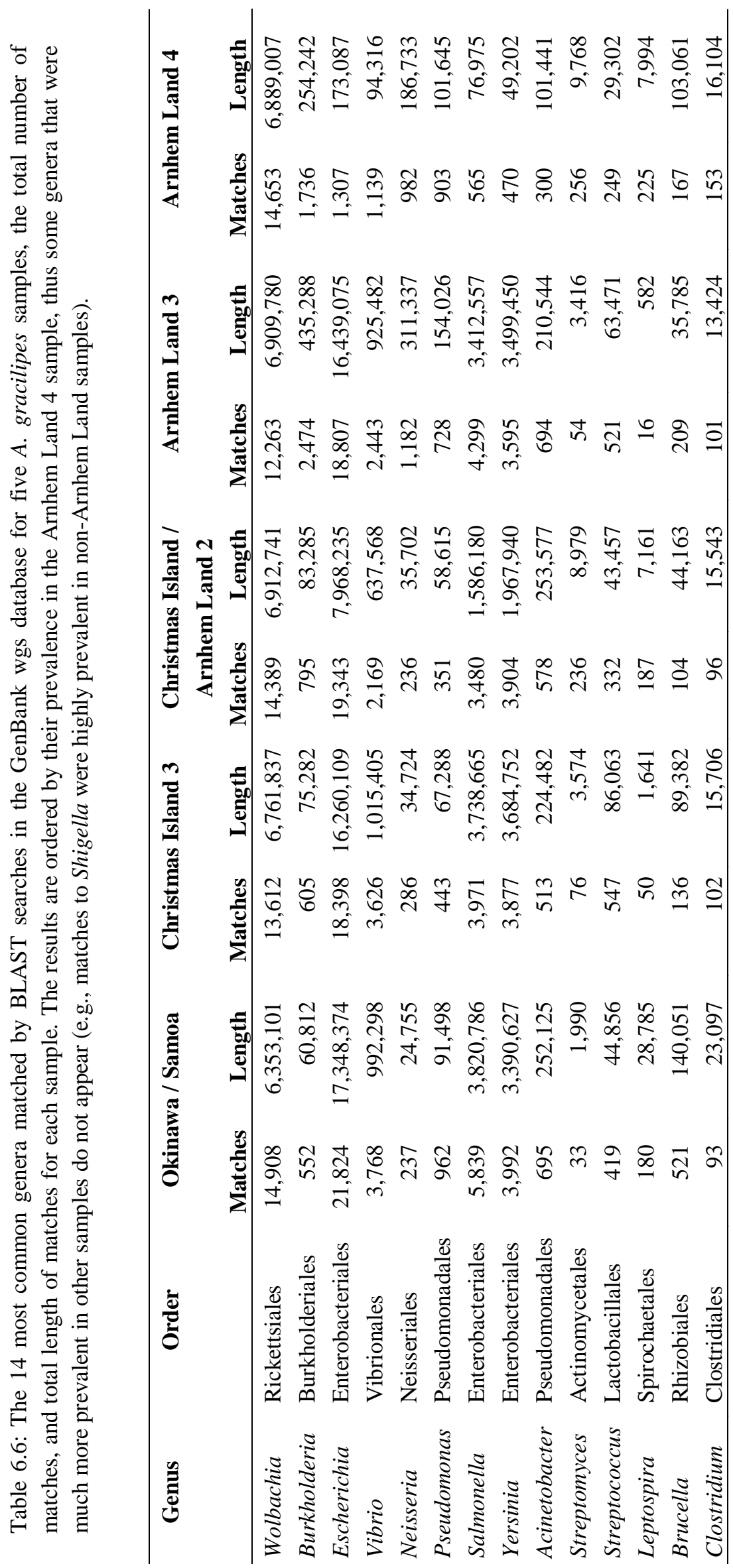




\section{Chapter 6}

\subsubsection{GenBank other_genomic database composition}

The results for the other_genomic database were similar to the wgs database, with high numbers of matches to Wolbachia and Escherichia (Table 6.7). Other bacterial groups also received a high number of matches, including Vibrio (order Vibrionales) and a number of Enterobacteriales. Once again, matches to these groups were less prevalent in the Arnhem Land 4 sample (Fig. 6.5). Matches to the genus Burkholderia were found for all samples, but again the number of matches was more than two times greater in the Arnhem Land 3 and 4 samples than in other samples (Table 6.7).

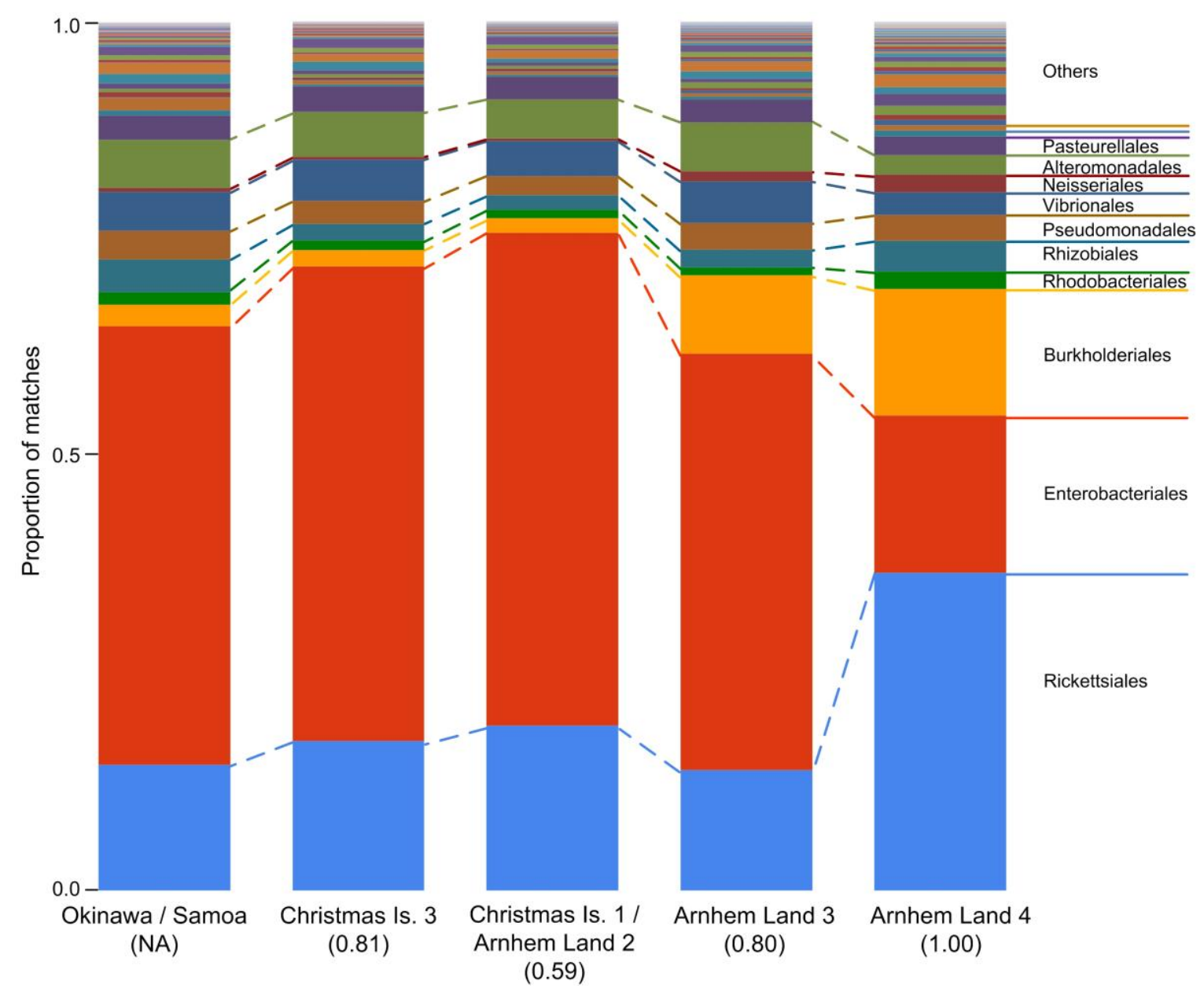

Fig. 6.5: Summary of BLAST matches to bacterial orders in the other_genomic database for five $A$. gracilipes samples. The figure in brackets below the sample name is the proportion of the sample that included ants from sites of low ant abundance (refer to Table 6.1 for details).As in Fig. 6.4 the distorted prevalence of Rickettsiales in the Arnhem Land 4 sample is owing to the low number of matches to most other orders. 


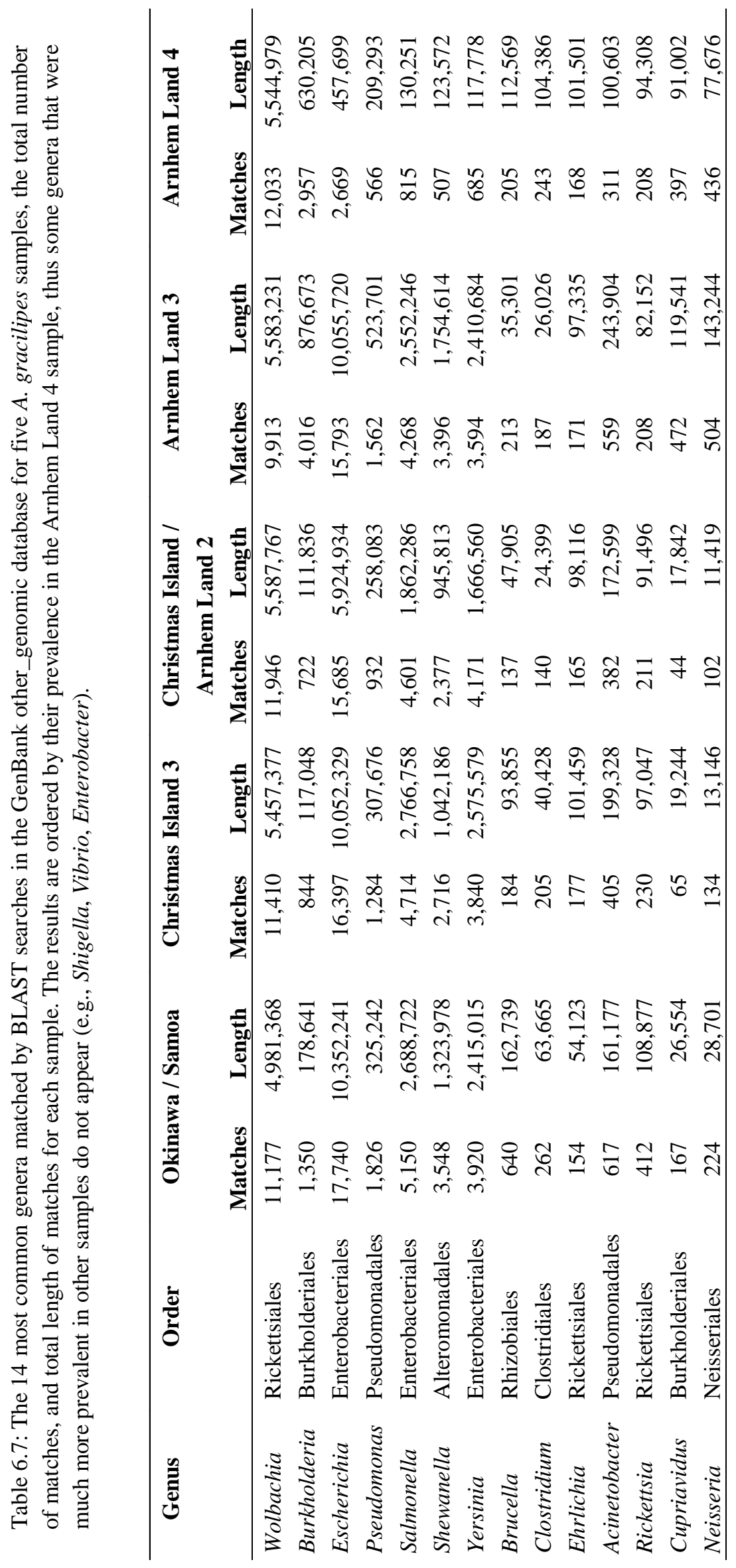




\section{Chapter 6}

\subsubsection{GenBank nt database composition}

The results for the nt database were similar to the wgs and other_genomic databases, with many matches to the Rickettsiales and Enterobacteriales orders (Fig. 6.6), and to the genera Wolbachia and Escherichia (Table 6.8). Matches to a number of bacterial groups were less prevalent in the Arnhem Land 4 sample than other samples (Table 6.8). Sequence matches to Burkholderia were higher in the Arnhem Land samples than in other samples, as were matches to Arsenophonus (order Enterobacteriales Table 6.8), which has previously been found in A. gracilipes (Sebastien et al. 2012).

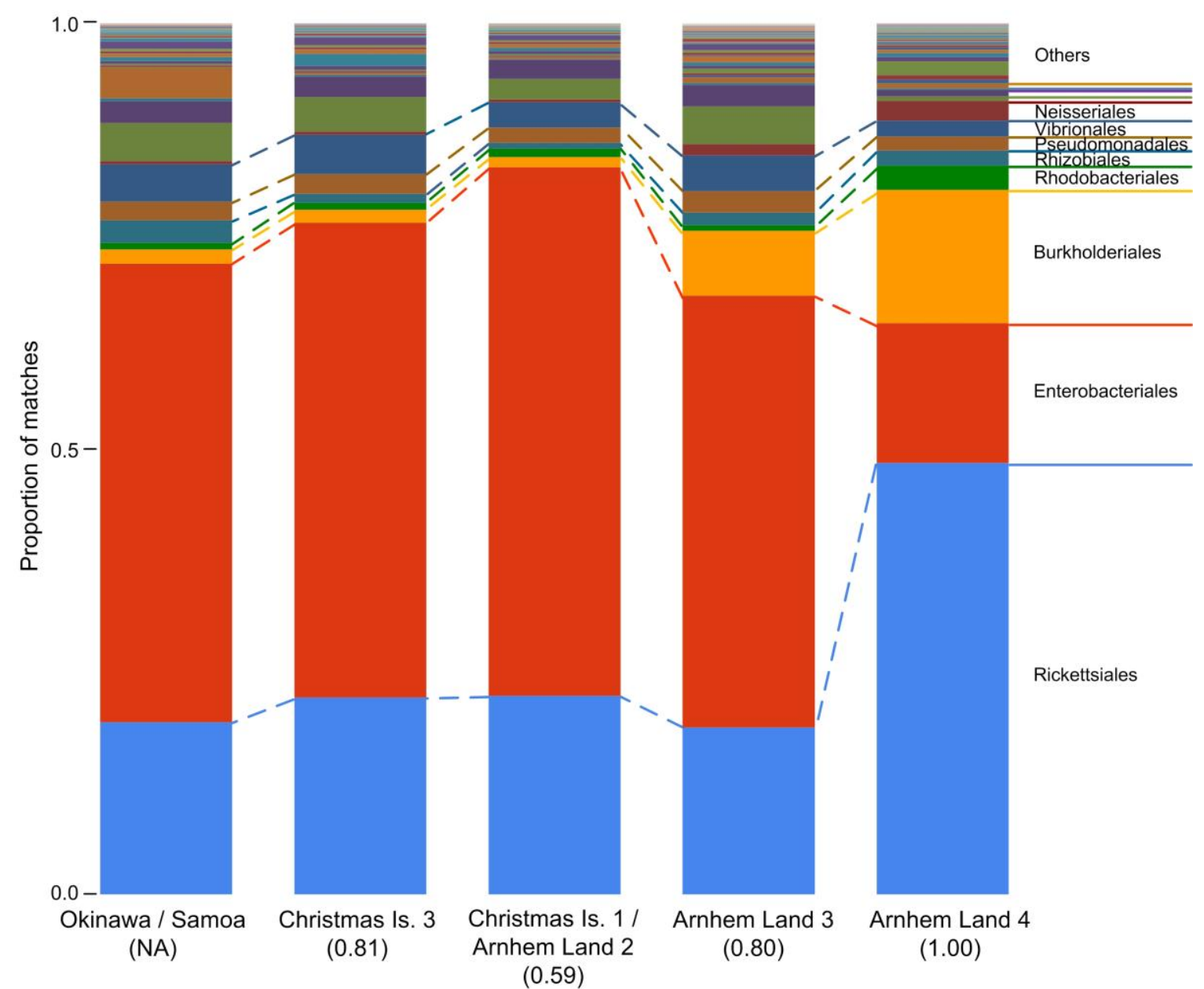

Fig. 6.6: Summary of BLAST matches to bacterial orders in the nt database for five $A$. gracilipes samples. The figure in brackets below the sample name is the proportion of the sample that included ants from sites of low ant abundance (refer to Table 6.1 for details). As in Fig 6.4 and 6.5, the distorted prevalence of Rickettsiales in the Arnhem Land 4 sample is owing to the low number of matches to most other orders. 


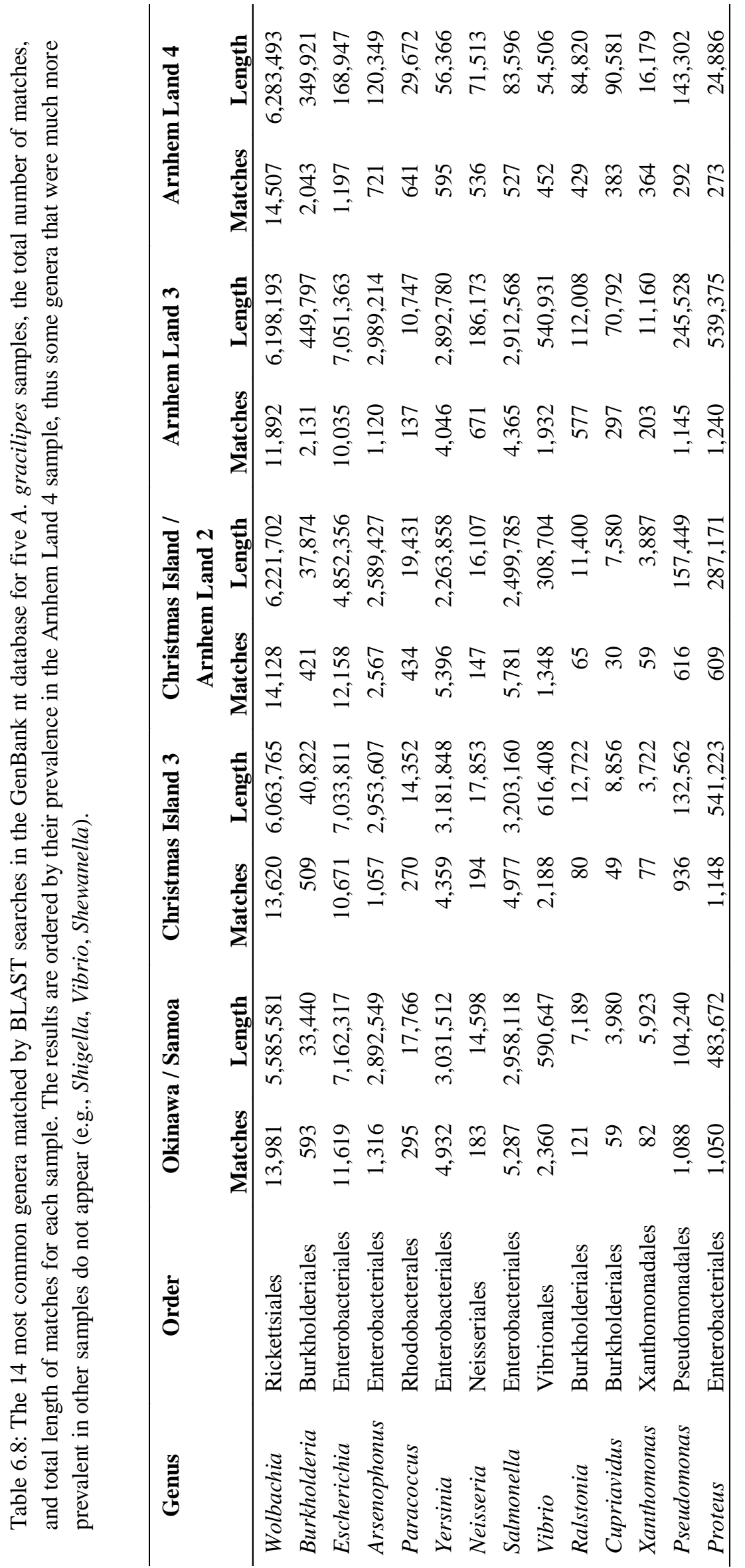




\section{Chapter 6}

\subsection{Discussion}

Animal populations introduced to new environments may bring with them a suite of beneficial or harmful endogenous symbionts and parasites. They may also encounter and assimilate new organisms. Population bottlenecks during the introduction may stochastically result in a loss of either or both beneficial and harmful symbionts, and endogenous symbiont and parasite composition may thus differ among introduced populations. In our study populations we found a low prevalence of eukaryote parasites of ants, such as fungi (Currie et al. 1999; Santos et al. 2004), microsporidia (Oi et al. 2008; Briano 2009; Valles et al. 2010), and nematodes (Yanoviak et al. 2008; Hoover et al. 2011), despite these groups being well represented in the databases. However, our study populations appeared to harbour considerable bacterial diversity, and differences among populations were apparent. The Arnhem Land samples exhibited results that were considerably different from the other samples among all four databases. The prevalence to groups common in other samples was much lower in the low abundance Arnhem Land 4 sample, with the exception of matches to sequences of the Burkholderiales order (and the genus Burkholderia).

\subsubsection{Community composition differences between databases}

We found differences in the results between the different databases. Genera that commonly received matches in some databases were absent from the results in others. For example, of the bacteria A. gracilipes is known to harbour (Wolbachia, Arsenophonus and Rhizobiales: Sebastien et al. 2012), Wolbachia sequences (order Rickettsiales) received no matches on the 16SMicrobial database, but had the most matches on the other databases. Arsenophonus (order Enterobacteriales) received matches for all samples but rarely in the 16SMicrobial database. This genus was absent from the wgs and other_genomic database results, but was one of the most prevalent results in the nt database. Rhizobiales was among the more commonly matched bacterial groups in all databases. However, matches to Rhizobiales occurred at a much lower prevalence than other orders except in the 16SMicrobial database. 
The reason for the differences in results among the databases is owing to differences in the data lodged in them. This potential bias may have resulted in the over-representation of some groups in the results. For example, organisms known to affect human health are likely to be over-represented. We excluded model-organism specific databases to minimise this effect. By chance, we also may have failed to detect some bacteria in the $16 \mathrm{~S}$ database if our short-read sequences did not include the $16 \mathrm{~S}$ region for any of these groups. However, the large amount of sequence data generated suggests this may be unlikely. Clearly the absence of matches to a particular group in a single database is not an indication that it is absent from the sample. We obtained more meaningful results from all the databases combined, rather than a single database. Our interpretation of the results attempts to take these issues into account.

\subsubsection{The role of Burkholderiales and associated symbionts}

Burkholderia species are among the most abundant bacteria in the environment, and are divided into two taxonomically distinct groups: one group whose members are pathogenic and another whose members are beneficial (Suárez-Moreno et al. 2012). Although some species are pathogenic in humans and other animals (Coenye and Vandamme 2003; Valvano et al. 2005), other species are plant-associated, both freeliving in the soil among roots, and as endosymbionts or phytopathogens (Coenye and Vandamme 2003; Janssen 2006; Omarjee et al. 2008). Species of Burkholderia are not known to have pathogenic relationships with insects, although their ecological roles are often uncertain (Vandamme et al. 2007a). Burkholderia are common beneficial gut symbionts of insects (Compant et al. 2008). Beneficial relationships between species of Burkholderia and insects include assistance with nutrition (van Borm et al. 2002; Kikuchi et al. 2005), and antibiotic protection (Santos et al. 2004; Vandamme et al. 2007b). 


\section{Chapter 6}

Members of the Burkholderiales have been detected among a number of ant species, and are among a group of endogenous bacteria assumed to be symbionts that facilitate herbivory (Russell et al. 2009). Other bacterial groups suggested to be herbivoryassociated among ants include members of the Rhizobiales, Pseudomonadales, Xanthomonadales and Verrucomicrobiales orders (Russell et al. 2009). Russell et al. (2009) screened a single A. gracilipes ant for these bacteria, with negative results. However, the prevalence of symbionts appears to vary among A. gracilipes populations (Sebastien et al. 2012; this study), so the Russell et al. (2009) study may have been unlikely to detect these organisms from a single sample, particularly if the symbionts are obtained facultatively. Of the other groups found to be associated with herbivory in ants (Russell et al. 2009), we found Rhizobiales, Pseudomonadales and Xanthomonadales to be common in many of our results. Verrucomicrobiales were also suggested by Russell et al. (2009) to be associated with herbivory. We found Verrucomicrobiales in the wgs, other_genomic and nt database results, but at such extremely low prevalence as to potentially be owing to sequence similarity, rather than a true indicator of prevalence.

The role of Burkholderia-related bacteria in A. gracilipes requires further attention before conclusion can be made regarding their function. Although A. gracilipes often dominates novel carbohydrate resources from plants directly (Lester and Tavite 2004; Savage et al. 2009), or indirectly (Abbott and Green 2007), it is also a generalist omnivore, that feeds on other insects, spiders, crabs and birds (Green et al. 1999). While it may seem unusual that an omnivorous ant such as $A$. gracilipes would require herbivory related symbionts, omnivorous ants may use symbionts to enhance their nutrition (Feldhaar et al. 2007). Moreover, as well as enhancing nitrogen recycling, Burkholderia species may function specifically to produce antibiotics as a protective function, as found in the leaf-cutting ant Atta sextens rubropilosa (Santos et al. 2004). Horizontal transmission of Burkholderia is common (Kikuchi et al. 2005), and the bacterium may be obtained from the soil (Santos et al. 2004). Additionally, Streptomyces are used by fungus-growing ants to protect against infection by fungal parasites (Currie et al. 1999). The Arnhem Land 4 sample had a much higher prevalence of matches to the order Actinomycetales, and to the Streptomyces genus within this group. We speculate that it is possible that the A. gracilipes population from which the Arnhem Land 4 sample 
was taken may have been infected by other organisms (perhaps fungi), and the facultative uptake of (potentially) antibiotic-producing bacteria from the soil may have been a response to infection. The lower prevalence of other bacteria in this sample that may be mutualistic suggests a hypothesis of fungal infection might be plausible. Although fungal and other eukaryote parasite sequences did not figure strongly in our results, when a population has declined due to disease, the extinction of the pathogen may precede that of the host (De Castro and Bolker 2005).

Alternatively, the species of Burkholderiales in A. gracilipes may be pathogenic members of the group. A number of species in the order Burkholderiales are pathogenic (Brogden 2009), and may consume antibiotics (Dantas et al. 2008) rather than produce them. However, this hypothesis is perhaps unlikely given the absence of documented pathogenic relationships between Burkholderiales and insects (Vandamme et al. 2007a).

\subsubsection{The role of Rickettsiales}

The order Rickettsiales includes the common insect symbiont Wolbachia, and its close relative Rickettsia. These bacteria are known to manipulate host sex-ratios in favour of females (Gherna et al. 1991; Engelstädter and Hurst 2009; Himler et al. 2011), which in some cases may have positive effects on the fitness of the host by increasing abundance and spread (Himler et al. 2011). However, Wolbachia may reduce fitness among ants (Wenseleers et al. 2002), and a number of invasive ant species have exhibited a loss of Wolbachia in the introduced range (Shoemaker et al. 2000; Tsutsui et al. 2003a). Although the loss of these bacteria may contribute to the invasion success of the host, the function of common insect endosymbionts such as Wolbachia in ants is unclear and may be context dependent (Zientz et al. 2005).

Both Wolbachia and Rickettsia featured in our results but were no more prevalent in any population. While it seems unlikely that either of these groups contributed to differences among our study populations, the role of Wolbachia and Rickettsia in A. gracilipes populations is of interest. Wolbachia has been detected previously in $A$. gracilipes at high prevalence (Sebastien et al. 2012). A high level of Wolbachia infection in the introduced range may be maintained through the genetic cleansing of incompatible strains (Wenseleers et al. 1998; Merçot and Poinsot 2009), which has been suggested for 


\section{Chapter 6}

A. gracilipes (Sebastien et al. 2012). High prevalence of Wolbachia has been attributed to the virus protection it affords (Hedges et al. 2008; Teixeira et al. 2008), which may be the case for A. gracilipes (Sebastien et al. 2012). In addition, Wolbachia may spread more easily in species that experience repeated extinction and recolonisation because founder events may allow the frequencies of infection to exceed unstable levels (Turelli and Hoffmann 1995; Tsutsui et al. 2003a). This scenario may be applicable to A. gracilipes, which appears to experience dynamic population changes (Haines and Haines 1978b; Abbott 2006b; B. Hoffmann \& P. Green, personal communication; Chapter 3; Chapter 5).

Wolbachia may also possibly affect the reproductive mode of A. gracilipes, as in some insects it can cause parthenogenetic production of females (Stouthamer et al. 1993). If Wolbachia has effects on reproduction in A. gracilipes it may contribute to the enigmatic reproductive mode of the species (Drescher et al. 2007; Chapter 2). Although to date no evidence has been found that Wolbachia affect reproduction among ant species (Wenseleers and Billen 2000; Keller et al. 2001), Wolbachia may be associated with a wider range of phenotypic effects on the host than previously assumed (Feldhaar 2011).

\subsubsection{Enterobacteriales and other common bacterial groups}

Enterobacteriales featured commonly in our results. However, our Arnhem Land population had a lower prevalence of sequence matches to this order than other populations. Arsenophonus, a member of the Enterobacteriales known to occur in $A$. gracilipes (Sebastien et al. 2012), was among the more prevalent sequences matched to in the nt database. Although the function of this bacterium is not known in A. gracilipes or other ants, Arsenophonus is known to modify the sex-ratio of host Hymenopterans (Gherna et al. 1991). 
The Enterobacteriaceae family to which Arsenophonus belongs includes a number of members that are beneficial symbionts of ants and other insects, such as Buchnera (Buchner 1965), Blochmannia (Zientz et al. 2005; de Souza et al. 2009), Wigglesworthia (Heller 2011), Regiella, Hamiltonella and Serratia (Oliver et al. 2010; Tsuchida et al. 2010). Arsenophonus-like species are also common symbionts of aphids (Russell and Moran 2005), and whiteflies (Thao and Baumann 2004). Clearly, many bacteria, including Arsenophonus and Wolbachia, may have differing phenotypic effects in different host species, and these effects are beneficial in specific cases and harmful in others.

Among the other groups commonly encountered in our results, were the orders Rhodospirillales, Sphingomonadales, Vibrionales, Caulobacterales, Pasteurellales and Alteromonadales. These groups are large with diverse characteristics (Kersters et al. 2006). As the prevalence of matches to these groups did not vary a great deal among most populations, and may thus reflect a 'noisy' dataset, investigating their function further may provide little gain. However, if the majority of endogenous bacteria are not pathogenic, and are in fact beneficial, treatment with antibiotics may result in population declines (Mittler 1971; Feldhaar et al. 2007).

\subsection{Conclusion}

Our preliminary metagenomic study of A. gracilipes populations found no significant eukaryote parasites, but a diversity of bacteria among A. gracilipes populations. Many bacterial groups were detected that were previously unknown in the species. Several of these are candidates for further study, which may shed light on the effects of endogenous parasites and symbionts on the population dynamics of the species. 


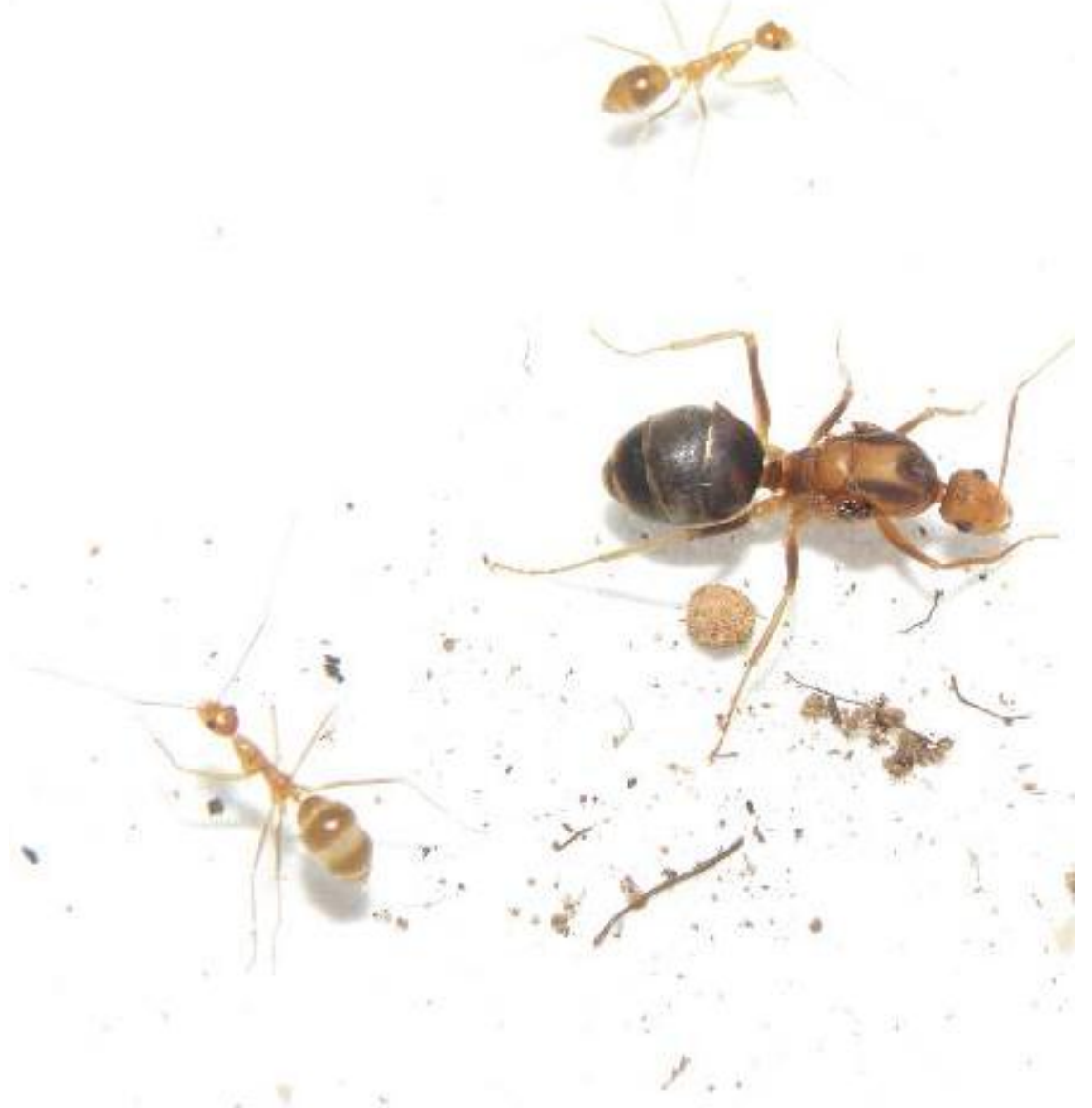




\section{Chapter 7. General discussion}

\subsection{Summary}

A key factor contributing to invasion success is the ability of an introduced population to reach sufficient abundance to persist, spread, and alter or dominate the recipient biological community (Ehrlich 1986; Crawley 1987; Mack et al. 2000). Genetic diversity is one factor that contributes to population dynamics, but has important ramifications for biological fitness, and thus invasion success in the long term. My dissertation investigated genetic factors associated with variation in abundance of the invasive yellow crazy ant Anoplolepis gracilipes. I investigated five aspects that I hypothesised could contribute to variation in the abundance of this ant: 1) dependent lineage genetic caste determination with lineage ratio asymmetry, which could be associated with low abundance; 2) multiple introductions which could increase abundance via propagule pressure, or different sources (i.e., genetically different colonies) with different levels of abundance; 3) differences in microsatellite genetic diversity between populations with different levels of abundance; 4) a specific haplotype associated with high abundance; 5) variation in endogenous parasite and symbiont diversity among populations which might be associated with variation in abundance.

My research suggests that genetic factors may contribute to the variation in abundance of A. gracilipes. I found no evidence for a dependent lineage genetic caste determination system in A. gracilipes, and thus abundance is not constrained by any lineage ratio asymmetry. Although ovaries were found in workers, no viable eggs were present. Based on this evidence, worker reproduction (either by production of males or clonal production of other workers) seems unlikely, or it may occur only seasonally. The reproductive mode of the species remains fascinating but enigmatic (Chapter 2). I also found no evidence that the variation in abundance of A. gracilipes in Arnhem Land was due to multiple introductions from different sources. By contrast, the population is a single heterogeneous supercolony that appears to be diverging, which is unusual in invasive ants (Chapter 3). While this divergence itself was not related to variation in abundance, genetic diversity was higher in more abundant nest clusters. These more abundant nest clusters were in 


\section{Chapter 7}

turn associated with lower native ant species diversity, and a difference in composition of the invaded ant community (Chapter 4). On Tokelau an earlier correlation identified between a specific haplotype and greater abundance was no longer detected, and although overall the distribution of the ant has increased, many A. gracilipes populations have declined or disappeared (Chapter 5). Variation in abundance among A. gracilipes populations in Arnhem Land was not obviously due to bacterial load on populations with low abundance, and I found no evidence of eukaryote parasites (Chapter 6). However, low abundance in A. gracilipes samples from Arnhem Land was correlated with lower microbial diversity overall, and higher prevalence of some bacterial groups, notably two that confer antibiotic properties (Chapter 6).

\subsection{Synthesis}

While dependent lineage GCD does not appear to be responsible for the variation in abundance of $A$. gracilipes in Arnhem Land (Chapter 2), the reproductive mode may contribute to the invasion success of the species if one or more castes or sexes are produced asexually. Recently it has been suggested that the ecological success of some invasive ants may be facilitated by clonal reproduction (Wasmannia auropunctata: Foucaud et al. 2009; Platythyrea punctata Cerapachys biroi, Pristomyrmex punctatus, Pyramica membranifera and Vollenhovia emeryi: Kellner and Heinze 2010, and Paratrechina longicornis: Pearcy et al. 2011). Asexual reproduction is a trait that evolved in the native range and is expressed more strongly, and selected for, in the invaded range of W. auropunctata (Foucaud et al. 2010). Although the native range of P. longicornis has not been studied it is suggested that the trait has 'pre-adapted' the ant to enable invasion success, by requiring few propagules for establishment (Pearcy et al. 2011). By contrast, if we assume that asexual reproduction does contribute to the variation in abundance of $A$. gracilipes, then it appears local abundance may be facilitated by a greater number of clonal lineages, as indicated by the higher genotypic diversity found in more abundant nest clusters in Arnhem Land (Chapter 4). This suggests that postestablishment, a larger propagule size (i.e., a greater diversity of reproductive individuals) might favour greater abundance than a small propagule size. Thus, even if the $A$. gracilipes reproduction mode involves asexuality, which could contribute to initial 
colonisation success, the reproductive mode may not contribute directly to the on-going persistence or spread of the ant.

The population divergence I detected (Chapter 3), together with the positive association between genotypic diversity and abundance (Chapter 4), may have implications for the long-term success of A. gracilipes in Arnhem Land. Population divergence may result in a loss of genetic diversity and decreased abundance over time. Anoplolepis gracilipes has been in Arnhem Land since at least the early 1980s. Nest clusters that were detected at that time which were at high abundance (Young et al. 2001) are now relatively less abundant than nest clusters at other sites (Chapter 4). Thus it appears that population divergence involving colony fragmentation (Chapter 3) may be associated with genetic bottlenecks that result in less abundant colonies with lower genetic diversity (Chapter 4).

Competition with native ant species may also further contribute to population fragmentation. The relationship between A. gracilipes and differences in the ecological community are density-dependent (Hill et al. 2003; Abbott 2005; Abbott et al. 2007; Lester et al. 2009; Hoffmann and Saul 2010; Drescher et al. 2011; Chapter 4). Anoplolepis gracilipes is unlikely to be able to compete with dominant ant species when the former are at low densities. In addition, A. gracilipes often occupies a dominant ecological position only in the absence of other highly aggressive and dominant species (Greenslade 1971; Bos et al. 2008; Kirschenbaum and Grace 2008; Brühl and Eltz 2010). This is consistent with my findings in Chapter 4, where A. gracilipes abundance was more negatively associated with dominant and rare species than with common and subdominant species. The ability of invasive ants to compete with native ants only when the invaders are in high abundance is well known (Oliver et al. 2008; Sagata and Lester 2009), which suggests that Allee effects (Allee 1931) may be mediated by competitive interactions among ant species. Thus, in areas with species-rich native ant communities such as those in Arnhem Land, A. gracilipes may not be an effective competitor at low abundance, which may further contribute to population fragmentation.

Although genetic bottlenecks generally, or specifically in nestmate recognition loci, may promote unicoloniality and thus the increased abundance of invasive ants (Suarez et al. 1999; Tsutsui et al. 2000; Giraud et al. 2002), post-introduction genetic bottlenecks 


\section{Chapter 7}

may also affect the dynamics of unicoloniality. Unicoloniality is thought to be an evolutionary dead-end, with the extinction of unicolonial species predicted to occur via kin-selected evolutionary mechanisms, including the development of finer-scale kin discrimination within the supercolony (Helanterä et al. 2009). This is consistent with the hypothesis of Drescher et al. (2010), together with my results (Chapters 3 and 4), which suggest that the loss of genetic diversity through serial bottlenecks may contribute to the breakdown of unicolonial populations by inducing fragmentation, and perhaps also decline at a local scale over ecological timeframes measured in decades rather than centuries. Such fragmentation potentially increases intra-specific aggression (Drescher et al. 2010), resulting in reduced colony sizes, and diminished competitive ability relative to native ants. Even if populations do not decline to the point of regional extinction, competition with native ant communities may result in alternative stable states of communities dominated by A. gracilipes in some cases, and native dominant ants in other cases (i.e., an ant mosaic: Leston 1973). This ant mosaic pattern evidently applies to the distribution of A. gracilipes in Borneo, for example (Pfeiffer et al. 2008), which is also consistent with the observation that colonies in Borneo (Drescher et al. 2010) are much smaller than the colony in Arnhem Land (Chapter 4).

Under the scenario described above, population bottlenecks may eventually result in local extinctions. A number of invasive ants show unpredictable, unexplained population declines (Greenslade 1971; Haines and Haines 1978b; Morrison 2002; Cooling et al. 2011). Serial population genetic bottlenecks may result in an extinction vortex, where the population declines to a size below which it can ecologically function (Gilpin and Soulé 1986). A hypothesis of population fragmentation, reduced genetic diversity and eventual decline is, however, based on the assumption that there is a persistent relationship between abundance and genetic diversity. Although higher genetic diversity may confer a number of short-term benefits (Cole and Wiernasz 1999; Tarpy 2003; Mattila and Seeley 2007; Oldroyd and Fewell 2007), particularly enhanced immune function (Sherman et al. 1988; Shykoff and Schmid-Hempel 1991; Keller 1995; Tarpy 2003; Ugelvig et al. 2010), the mechanisms that maintain genetic diversity, and how much higher genetic diversity needs to be to confer these benefits is not known in A. gracilipes. In addition, the long term adaptive benefits, and dynamics of the association between genetic diversity and abundance over time are unknown. 
It is likely that colony fragmentation of $A$. gracilipes populations would not occur on small islands such as Tokelau, for example. On these islands dispersal is limited, ant colonies are likely to remain in sympatry, and the likelihood of re-invasion appears high (Chapter 5). In addition, competition with dominant native ants is unlikely to have an effect on islands such as Tokelau, as the ant fauna is cosmopolitan and comprises many introduced species (Abbott et al. 2006).

Based on what we know of the population dynamics of A. gracilipes on Tokelau (Lester and Tavite 2004; Abbott et al. 2007; Lester et al. 2009; Chapter 5), some aspects of the population dynamics of the ant there may be somewhat similar to that in Arnhem Land. On Tokelau the more recent haplotype D populations declined, while the older haplotype A populations appeared to remain relatively constant at lower abundance (Chapter 5). This mirrors the finding that the earliest detections in Arnhem Land, which were highly abundant (Young et al. 2001) are no longer abundant, while the abundance of the ant in more recently invaded sites appears higher (Chapter 4). The density-dependent ecological effects of other invasive ants can also be higher shortly after arrival, and then decline (Morrison 2002). Invasive species generally may experience an acute phase during which their abundance and effects are most severe, followed by a long chronic phase of stability (Strayer et al. 2006). Clearly, understanding these dynamics requires further work.

Although the metagenomic investigation in Chapter 6 was preliminary, and was qualitative rather than quantitative, it does raise interesting questions regarding the potential role of endosymbionts in the variation in abundance and dynamics of $A$. gracilipes populations. I had initially hypothesised that parasites or pathogens could be responsible for the low abundance (presumably through population decline) of $A$. gracilipes in Arnhem Land. If bacterial pathogens were responsible for variation in abundance, bacterial load would be much higher in the groups with lower abundance. In addition, if pathogenic bacteria were responsible for population declines bacterial diversity would differ in A. gracilipes populations of low abundance, and that pathogenic organisms would be more prevalent in these populations. My results suggested that the opposite was the case for both hypotheses. The most common bacterial species in samples from Arnhem Land with low abundance were Burkholderia. Burkholderia species fall 


\section{Chapter 7}

into two groups: some that are widely known as pathogenic to humans and animals, and some are beneficial to plants (Suárez-Moreno et al. 2012), and insects including ants (van Borm et al. 2002; Santos et al. 2004; Kikuchi et al. 2005; Vandamme et al. 2007b; Compant et al. 2008). There are no reports of pathogenic Burkholderia in insects. Thus, the function of Burkholderia-like species in A. gracilipes is clearly worthy of further consideration.

Given the potential importance of beneficial endosymbionts such as Burkholderiales to enhance nutrition among ant species (Currie et al. 1999; Feldhaar et al. 2007; Russell et al. 2009; Feldhaar 2011), the potential use of novel carbohydrates deserves closer attention, in light of the findings of Chapter 6. Although the presence of Acacia spp. was investigated (Chapter 4), no estimates were made of possible differences in usage of Acacia extra floral nectaries by $A$. gracilipes. One of the roles of candidate endosymbionts such as Burkholderiales (Chapter 6) is the provision of nutritional upgrading in non-herbivorous ant species (Feldhaar et al. 2007). Predatory invasive ants are known to shift their diet from animals to plants in the introduced range as protein resources are exhausted (Tillberg et al. 2007), and depletion of protein resources had been suggested as a possible, but untested, reason for the decline of A. gracilipes on Mahé in the Seychelles (Haines and Haines 1978b). Thus, endosymbionts such as Burkholderiales may possibly be used facultatively by $A$. gracilipes to facilitate uptake of plant-based nutrients when protein resources are locally depleted.

In ants, a positive relationship exists between genetic diversity and immunity (Sherman et al. 1988; Shykoff and Schmid-Hempel 1991; Keller 1995; Tarpy 2003; Ugelvig et al. 2010). Social insects, including ants, have fewer immune genes than other insects, and thus their populations typically have naturally high pathogen loads (SchmidHempel 1998). Pathogen spread is facilitated by the social organisation of these insects, owing to aggregations of close relatives (Sherman et al. 1988). Consequently, social insects employ a number of behavioural and physiological mechanisms to enhance disease resistance (Cremer and Sixt 2009). Enhanced disease resistance through improved immune defence has been hypothesised to drive increased genetic diversity within social insect colonies, favouring colonies with a higher number of reproductives (i.e., multiple queens reproduce [polygyny], and / or queens mate with multiple males [polyandry]), and 
consequently lower parasite loads (Liersch and Schmid-Hempel 1998; Schmid-Hempel and Crozier 1999; Tarpy 2003). While both low genotypic diversity (Chapter 4) and low bacterial diversity (Chapter 6) were associated with low abundance of A. gracilipes in Arnhem Land, it is not possible to conclude that there is a direct or interactive relationship between genetic diversity and bacterial diversity. However, given the apparent benefits of genetic diversity, and possible effects of endogenous parasites, the combination of these factors would benefit from further study.

Without knowledge of the native range of A. gracilipes it is not possible to discover which differences may enhance or hinder establishment success in the introduced range. Although other members of the genus are found only in Africa, Madagascar, the Arabian peninsula and Réunion (Wetterer 2005), there have been suggestions that Asia may be within the native range of the species (Wetterer 2005; Drescher 2011; Sebastien et al. 2012; H. Feldhaar, personal communication). India has not been sampled for genetic analysis, although it has been suggested that this may ultimately prove to be the native range (Drescher 2011; H. Feldhaar, personal communication). Hypothetically, if the closest relatives, and potentially ancestors, of A. gracilipes were in Madagascar and Africa, the origin of A. gracilipes could be vicariant owing to the break-up of Gondwana (i.e., the out-of-India hypothesis: McKenna 1973). If this were the case, India would have been a natural point of origin for dispersal of the ants throughout Asia. The origin of ants pre-dates the break-up of Pangaea (Crozier et al. 1997; Moreau 2009). Formicinae (the sub-family to which A. gracilipes belongs) appear to have evolved $\sim 100 \mathrm{Ma}$ (Moreau et al. 2006), not long before the approximate separation of India and Madagascar from Gondwana $90 \mathrm{Ma}$ (Scortese 1997), so a vicariant origin is possible.

Differences in colony structure among A. gracilipes populations may enable inferences to be made regarding the native range. Invasive unicolonial ants often exhibit differences in colony structure in the introduced range compared to the native range, and a striking difference is colony size. For example, the diameter of Argentine ant colonies in their native range has been estimated to not exceed $2 \mathrm{~km}$, and colonies are frequently much smaller (Heller 2004). In the introduced ranges colony sizes are reported to be many orders of magnitude larger (Giraud et al. 2002; Corin et al. 2007; Suhr et al. 2009; van Wilgenburg et al. 2010). The extent of supercolonies in these ants are therefore much 


\section{Chapter 7}

greater in the introduced range (Suarez et al. 2008). Most invasions of $A$. gracilipes have been studied on relatively small islands, where the size that supercolonies can extend to is spatially limited (Haines and Haines 1978b; Feare 1999; Abbott 2006b; Abbott et al. 2007; Suwabe et al. 2009). These spatial limits clearly do not exist in Arnhem Land (Chapter 4). By contrast, other than my work the only study of A. gracilipes on a large scale has been in Borneo, where despite the absence of geographical constraints, genetically and behaviourally differentiated colonies span only a few hundred metres (Drescher et al. 2010). Colonies of this size are more akin to small polydomous colonies (Bourke and Franks 1995; Suarez et al. 2008), as observed in the native range of the Argentine ant (Tsutsui et al. 2000; Pedersen et al. 2006).

If a phenomenon of colony 'gigantism' in the invaded range, due to a loss of intraspecific aggression, is common among unicolonial invasive ants, this may be an indicator that Borneo is within the native range of A. gracilipes. Even on Christmas Island, the social structure appears very different to that in Borneo, whereby two supercolonies share an overlapping distribution throughout the entire island (Thomas et al. 2010). The allelic richness of individual A. gracilipes colonies in Borneo has not been reported (Drescher et al. 2007; Drescher et al. 2010; Drescher 2011), so we do not know if colonies in Borneo are more genetically diverse that those on Christmas Island (Thomas et al. 2010), or than those in Arnhem Land (Chapter 4). Based on these features it appears possible that Borneo is within the native range of $A$. gracilipes, as others have suggested (Wetterer 2005; Drescher 2011; Sebastien et al. 2012; H. Feldhaar, personal communication). Given the clear differences in colony structure between Arnhem Land (Chapter 4) and Borneo (Drescher et al. 2010), that appear to mirror differences between the native and introduced ranges in Argentine ants (Tsutsui et al. 2000; Giraud et al. 2002; Pedersen et al. 2006; Corin et al. 2007; Suhr et al. 2009; van Wilgenburg et al. 2010), more direct comparisons between A. gracilipes in Borneo (and India) and the introduced range may shed more light on the potential causes of the differences between the ranges. 


\subsection{Constraints and further research}

A number of further interesting avenues of research are possible in relation to the genetic factors contributing to the abundance and population dynamics of A. gracilipes. Many of these research directions require longer term research on the changes in population dynamics over time. During the course of my thesis I faced a number of constraints which also highlighted significant unanswered questions regarding the biology and ecology of this under-studied species.

As part of my work in Chapter 2, I originally attempted to determine whether clonal reproduction occurred in A. gracilipes. As lab facilities were not available in either in New Zealand or in Australia, I (somewhat naïvely in hindsight) attempted to determine statistically if the genotypes of workers and queens were consistent with clonal or sexual reproduction (primarily using the GenClone functions of Arnaud-Haond and Belkhir 2007). The results of these tests were mixed and asexual reproduction by queens or workers appeared no more likely than sexual reproduction by mated queens. I did not include these analyses in Chapter 2, however, as when a manuscript including these analyses was submitted, reviewers consistently pointed out that these questions could only be answered through experimental laboratory nests that allow the control of the parental relationships between individuals. I agreed with this assessment, and thus I modified Chapter 2 to focus only on DL GCD, a hypothesis I consider can be rejected based upon the evidence of a single queen lineage in Arnhem Land.

The social systems of ants have traditionally been viewed as families of sterile workers, all offspring of a single diploid mother queen mated to a single haploid male (Pamilo 1991; Heinze 2008), resulting in relatively low genetic diversity compared to species that are not haplo-diploid (Hedrick and Parker 1997). However, the diversity of reproductive systems that have been discovered among ants in recent decades means that very little can be assumed about the reproductive mode of a given species (Keller 2007; Heinze 2008). We lack knowledge of a number of aspects of A. gracilipes biology, and reproduction in particular, that relate directly to the genetic diversity of ant populations (Pamilo 1991). How many queens and males contribute to reproduction? Does the species mate only within the nest or do nuptial flights occur, and if they do, are they common or 


\section{Chapter 7}

rare events? Is there sex-biased dispersal? How does high heterozygote frequency among workers and homozygote frequency among queens contribute to fitness? How is worker exchange among nests mediated? What is the structure of nest clusters within the colony and how does this vary? How do these aspects vary in different parts of the invaded range, and how does this compare with the (unknown) native range? Some of these questions can perhaps be answered through field assessments. However, answering many of these fundamental questions requires experimental manipulation of colonies.

The genomic short-read-sequence dataset generated for this project could not be fully exploited due to a number of timing constraints regarding sequencing, and provision of computing resources. The results reflect the short amount of time that was available for the study once computing resources were in place. In addition to exploring metagenomic diversity, my original intent was to determine if genetic diversity differed between functional genes and neutral markers (i.e., bottlenecks may affect the diversity of functional genes less than neutral markers (Väli et al. 2008)), and to identify markers that may provide greater resolution of the genome (i.e., single nucleotide polymorphisms or SNP markers (Morin et al. 2004)). These types of markers might be particularly useful given the small number of existing polymorphic microsatellite markers for A. gracilipes, which as a result have limited use in determining fine-scale genetic structure (Koskinen et al. 2004). Illumina data may also be used to generate new microsatellite markers (Castoe et al. 2012).

Another use of the short-read-sequence dataset is to search for potential genes that may be related to variation in abundance (e.g., genes associated with functions such as insulin pathways, recognition or immunity). This latter aim may be more feasible now than when I started my thesis, as six ant genomes have been sequenced and annotated over the last year or so (Bonasio et al. 2010; Smith et al. 2011a; Smith et al. 2011b; Suen et al. 2011; Wurm et al. 2011), together with a number of other Hymenopteran genomes that have been more extensively annotated (Munoz-Torres et al. 2011). A number of candidate genes have also been identified for pest control of ants (Rodovalho et al. 2011). These include genes that are involved development and longevity, immunity, resistance to pathogens, pheromone function, behaviour and polysaccharide metabolism (Rodovalho et al. 2011). In addition, genes coding for defensin antimicrobial proteins, which are under 
positive selection in ants (Viljakainen and Pamilo 2008), and vitellogenin, which is involved in stress resilience and social organisation in honeybees (Amdam et al. 2012) may be among candidates that contribute to variation in abundance.

The metagenomic aspect of my study could be refined further. The variation in data publicly available on GenBank is likely to be a constraint, and there are other microbial database options including those that are publicly available (e.g., the RDP database used by Anderson et al. 2012) or privately held (e.g., the Microbial Diversity Analysis Service used by Ishak et al. 2011). Identifying, and correcting for, the bias in the GenBank databases (i.e., whether high numbers of matches are due to prevalence of particular organisms in the database) will also be useful. Further work on this aspect includes investigating ways of extracting more data from the many unused reads in the dataset by refining the assemblies (initially by varying the assembly parameters, including combining assemblies with different k-mer lengths [B. Schoenfeld personal communication]), and metagenome analyses using different bioinformatic approaches (e.g., different assemblers such as MEGAN (Huson et al. 2011), ABySS (Simpson et al. 2009), SOAP (Li et al. 2009) or PE-Assembler (Nuwantha Ariyaratne and Sung 2011)). Unused or unmapped reads are sometimes biologically meaningful, however, and can be due to viral sequences (Li et al. 2011), which may also prove a useful avenue to explore in relation to causes of population declines. Further analysis of the short-read-sequence dataset may be complicated, however, by the challenge of heterozygote genotypes that were, by necessity, used for sequencing. This 'phasing' problem perhaps contributes to the number of unused reads in the assemblies, and is a further challenge for which to investigate a solution.

The actual function of bacteria (and potential parasites) in A. gracilipes populations can only be assessed via controlled experiments. Experiments would also enable the direct assessment of any interaction between the genetic diversity of the host and its susceptibility to infection. If this were possible, a good sampling design would include multiple replicates from populations that are known to have declined together with those that have not, and comparisons with the native range (or at least colonies from possible candidate native ranges). Now that we have some idea of the types of bacteria that may be present in A. gracilipes, assays could be developed with specific primers to test for the 


\section{Chapter 7}

prevalence of these bacteria. Treatment with antibiotics and infection challenges would also be informative, and protocols are available for culturing Burkholderia (Vandamme et al. 2007a). However isolating and culturing other more general bacterial strains may prove more difficult.

\subsection{Conclusion}

A key factor in invasion success is the ability for a population to reach sufficient abundance to persist, spread, and alter or dominate the recipient biological community (Ehrlich 1986; Crawley 1987; Mack et al. 2000). Genetic diversity has important ramifications for biological fitness, and thus invasion success in the long term. The vast majority of studies of A. gracilipes to date have focussed on the ecological causes of the success of this invasive ant. Genetic factors play an important role in the success of other invasive ants, and this is the first study to directly investigate the possible role of multiple genetic factors in the variation in abundance, and thus differential invasion success of $A$. gracilipes. In addition, this is to my knowledge the first study to use Illumina short-readsequencing to investigate metagenomic diversity in an ant species. While my metagenomic work is only preliminary, and although all my thesis findings are essentially correlational rather than mechanistic, the study highlights a number of avenues of research into the causes of variation in A. gracilipes abundance, which may also be applicable more generally to other invasive ant species.

Together, my findings suggest that propagule pressure, reproductive mode and haplotype-specific effects do not appear to be associated with variation in A. gracilipes abundance. Even if clonal reproduction occurs in the species, it may assist establishment success rather than long term persistence. Other genetic factors I investigated do appear to be associated with variation in A. gracilipes abundance. Genotypic diversity was positively related to the abundance of A. gracilipes in Arnhem Land, and this relationship may be affected by population divergence through population bottlenecks. In addition, differences in bacterial diversity may also be associated with variation in abundance among populations. Although genetic factors are often implicated in the successful establishment of invasive species, my thesis demonstrates that genetic factors may also be associated with post-establishment population dynamics. 


\section{References}

Abbott, K. L. (2004). Alien ant invasion on Christmas Island, Indian Ocean: the role of ant-scale associations in the dynamics of supercolonies of the yellow crazy ant, Anoplolepis gracilipes, $\mathrm{PhD}$ dissertation, Monash University, Melbourne, Australia.

Abbott, K. L. (2005). Supercolonies of the invasive yellow crazy ant, Anoplolepis gracilipes, on an oceanic island: forager activity patterns, density and biomass. Insectes Sociaux 52, 266-273.

Abbott, K. L. (2006a). Challenging the yellow crazy ant, Anoplolepis gracilipes, on Tokelau; development and implementation of control options for invasive ants (a project supported by the Pacific Invasives Initiative). Report of an awareness and training programme; April-June, 2006.

Abbott, K. L. (2006b). Spatial dynamics of supercolonies of the invasive yellow crazy ant, Anoplolepis gracilipes, on Christmas Island, Indian Ocean. Diversity and Distributions 12, 101-110.

Abbott, K. L., Greaves, S. N. J., Ritchie, P. A. \& Lester, P. J. (2007). Behaviourally and genetically distinct populations of an invasive ant provide insight into invasion history and impacts on a tropical ant community. Biological Invasions 9, 453-463.

Abbott, K. L. \& Green, P. T. (2007). Collapse of an ant-scale mutualism in a rainforest on Christmas Island. Oikos 116, 1238-1246.

Abbott, K. L., Sarty, M. \& Lester, P. J. (2006). The ants of Tokelau. New Zealand Journal of Zoology 33, 157-164.

Akaike, H. (1973). Information theory as an extension of the maximum likelihood principle. Proceedings of the Second International Symposium on Information Theory. (ed. by B. N. Petrov and F. Csàki), pp. 267-281, Academiai Kiàdo, Budapest, Hungary.

Allee, W. C. (1931). Animal Aggregations: A Study in General Sociology, University of Chicago Press, Chicago, IL.

Allendorf, F. W. (1986). Genetic drift and the loss of alleles versus heterozygosity. Zoo Biology 5 , $181-190$.

Allendorf, F. W. \& Lundquist, L. L. (2003). Introduction: population biology, evolution, and control of invasive species. Conservation Biology 17, 24-30.

Altermatt, F. \& Ebert, D. (2008). Genetic diversity of Daphnia magna populations enhances resistance to parasites. Ecology Letters 11, 918-928.

Amdam, G. V., Fennern, E. \& Havukainen, H. (2012). Vitellogenin in honey bee behavior and lifespan. Honeybee Neurobiology and Behavior. (ed. by C. G. Galizia, D. Eisenhardt and M. Giurfa), pp. 17-29. Springer, Netherlands. 


\section{References}

Andersen, A. N. (1995). A classification of Australian ant communities, based on functional groups which parallel plant life-forms in relation to stress and disturbance. Journal of Biogeography 22, 15-29.

Andersen, A. N. (2000). The Ants of Northern Australia: A Guide to the Monsoonal Fauna, CSIRO Publishing, Collingwood, Australia.

Andersen, A. N. \& Hoffmann, B. D. (2011). Conservation value of low fire frequency in tropical savannas: ants in monsoonal northern Australia. Austral Ecology 36, 497-503.

Anderson, K. E., Hölldobler, B., Fewell, J. H., Mott, B. M. \& Gadau, J. (2006). Population-wide lineage frequencies predict genetic load in the seed-harvester ant Pogonomyrmex. Proceedings of the National Academy of Sciences of the United States of America 103, 13433-13438.

Anderson, K. E., Russell, J. A., Moreau, C. S., Kautz, S., Sullam, K. E., Hu, Y. I., Basinger, U., Mott, B. M., Buck, N. \& Wheeler, D. E. (2012). Highly similar microbial communities are shared among related and trophically similar ant species. Molecular Ecology 21, 2282-2296.

Anderson, M. J., Gorley, R. N. \& K.R., C. (2008). PERMANOVA+ for Primer: guide to software and methods, PRIMER-E, Plymouth.

Andrews, S. (2011). FastQC v 0.9.6, Babraham Bioinformatics. Available from http://www.bioinformatics.bbsrc.ac.uk/projects/fastqc/.

Arnaud-Haond, S. \& Belkhir, K. (2007). Genclone: a computer program to analyse genotypic data, test for clonality and describe spatial clonal organization. Molecular Ecology Notes 7, 1517.

Badano, E. I., Regidor, H. A., Núñez, H. A., Acosta, R. \& Gianoli, E. (2005). Species richness and structure of ant communities in a dynamic archipelago: effects of island area and age. Journal of Biogeography 32, 221-227.

Baker, G. (1976). The seasonal life cycle of Anoplolepis longipes (Jerdon) (Hymenoptera: Formicidae) in a cacao plantation and under brushed rain forest in the northern district of Papua New Guinea. Insectes Sociaux 23, 253-261.

Baker, H. G. (1965). Characteristics and modes of origin of weeds. The Genetics of Colonizing Species. (ed. by H. G. Baker and G. L. Stebbins), pp. 147-168. Academic Press, New York, NY.

Baker, H. G. (1995). Aspects of the genecology of weeds. Genecology and Ecogeographic Races. (ed. by A. R. Kruckeberg, R. B. Walker and A. E. Leviton), pp. 189-224. Pacific Division of the American Association for the Advancement of Science, San Francisco, CA.

Balloux, F., Lehmann, L. \& de Meeûs, T. (2003). The population genetics of clonal and partially clonal diploids. Genetics 164, 1635-1644.

Barton, N. H. \& Charlesworth, B. (1984). Genetic revolutions, founder effects, and speciation. Annual Review of Ecology and Systematics 15, 133-164.

Bartram, A. K., Lynch, M. D. J., Stearns, J. C., Moreno-Hagelsieb, G. \& Neufeld, J. D. (2011). Generation of multimillion-sequence $16 \mathrm{~S}$ rRNA gene libraries from complex microbial 
communities by assembling paired-end Illumina reads. Applied and Environmental Microbiology 77, 3846-3852.

Blight, O., Renucci, M., Tirard, A., Orgeas, J. \& Provost, E. (2010). A new colony structure of the invasive Argentine ant (Linepithema humile) in Southern Europe. Biological Invasions 12, 1491-1497.

Boland, C. R. J., Smith, M. J., Maple, D., Tiernan, B., Reeves, R. B. R. \& Napier, F. (2011). Helibaiting using low concentration fipronil to control invasive yellow crazy ant supercolonies on Christmas Island, Indian Ocean. Island Invasives: Eradication and Management. (ed. by C. R. Veitch, M. N. Clout and D. R. Towns), pp. 152-156, IUCN, Gland, Switzerland.

Bonasio, R., Zhang, G., Ye, C., Mutti, N. S., Fang, X., Qin, N., Donahue, G., Yang, P., Li, Q., Li, C., Zhang, P., Huang, Z., Berger, S. L., Reinberg, D., Wang, J. \& Liebig, J. (2010). Genomic comparison of the ants Camponotus floridanus and Harpegnathos saltator. Science 329, 10681071 .

Bos, M., Tylianakis, J., Steffan-Dewenter, I. \& Tscharntke, T. (2008). The invasive yellow crazy ant and the decline of forest ant diversity in Indonesian cacao agroforests. Biological Invasions 10, 1399-1409.

Bourke, A. F. G. (1988). Worker reproduction in the higher eusocial Hymenoptera. The Quarterly Review of Biology 63, 291-311.

Bourke, A. F. G. \& Franks, N. R. (1995). Social Evolution in Ants, Princeton University Press, Princeton, NJ.

Brandt, M., Van Wilgenburg, E. \& Tsutsui, N. D. (2009). Global-scale analyses of chemical ecology and population genetics in the invasive Argentine ant. Molecular Ecology 18, 9971005.

Briano, J. A. (2009). Long-term studies of the red imported fire ant, Solenopsis invicta, infected with the microsporidia Vairimorpha invictae and Thelohania solenopsae in Argentina. Environmental Entomology 34, 124-132.

Brogden, K. A. (2009). Cytopathology of pathogenic prokaryotes. Ultrastructural Pathology: The Comparative Cellular Basis of Disease. (ed. by N. F. Cheville), pp. 424-523. Wiley-Blackwell, Online Library.

Brühl, C. \& Eltz, T. (2010). Fuelling the biodiversity crisis: species loss of ground-dwelling forest ants in oil palm plantations in Sabah, Malaysia (Borneo). Biodiversity and Conservation 19, 519-529.

Buchner, P. (1965). Endosymbiosis of Animals with Plant Microorganisms, John Wiley \& Sons, New York, NY.

Burnham, K. P. \& Anderson, D. R. (2002). Model Selection and Inference: A Practical Information-theoretic Approach, Springer-Verlag Inc., New York, NY.

Caporaso, J. G., Lauber, C. L., Walters, W. A., Berg-Lyons, D., Lozupone, C. A., Turnbaugh, P. J., Fierer, N. \& Knight, R. (2011). Global patterns of 16S rRNA diversity at a depth of millions of sequences per sample. Proceedings of the National Academy of Sciences of the United States of America 108, 4516-4522. 


\section{References}

Carson, H. (1968). The population flush and its genetic consequences. Population Biology and Evolution. (ed. by R. C. Lewontin), pp. 123-137. Syracuse University Press, Syracuse, NY.

Castoe, T. A., Poole, A. W., de Koning, A. P. J., Jones, K. L., Tomback, D. F., Oyler-McCance, S. J., Fike, J. A., Lance, S. L., Streicher, J. W., Smith, E. N. \& Pollock, D. D. (2012). Rapid microsatellite identification from Illumina paired-end genomic sequencing in two birds and a snake. PLoS ONE 7, e30953.

Chakraborty, R. \& Nei, M. (1982). Genetic differentiation of quantitative characters between populations or species: I. Mutation and random genetic drift. Genetics Research 39, 303-314.

Cheverud, J. M. \& Routman, E. J. (1996). Epistasis as a source of increased additive genetic variance at population bottlenecks. Evolution 50, 1042-1051.

Clarke, K. R. \& Gorley, R. N. (2006). PRIMER v 6.1.11: user manual and tutorial, PRIMER-E, Plymouth.

Clarke, K. R. \& Warwick, R. M. (2001). Change in marine communities: an approach to statistical analysis and interpretation, PRIMER-E, Plymouth.

Clement, M., Posada, D. \& Crandall, K. (2000). TCS: a computer program to estimate gene genealogies. Molecular Ecology 9, 1657-1660.

Coenye, T. \& Vandamme, P. (2003). Diversity and significance of Burkholderia species occupying diverse ecological niches. Environmental Microbiology 5, 719-729.

Cohen, J. (1988). Statistical Power Analysis for the Behavioral Sciences, Erlbaum, Hillsdale, NJ.

Colautti, R. I., Grigorovich, I. A. \& MacIsaac, H. J. (2006). Propagule pressure: a null model for biological invasions. Biological Invasions 8, 1023-1037.

Colautti, R. I., Ricciardi, A., Grigorovich, I. A. \& MacIsaac, H. J. (2004). Is invasion success explained by the enemy release hypothesis? Ecology Letters 7, 721-733.

Cole, B. J. \& Wiernasz, D. C. (1999). The selective advantage of low relatedness. Science 285, 891-893.

Colwell, R. K. (2009). EstimateS: statistical estimation of species richness and shared species from samples, Version 8.2. User's Guide and application published at: http://purl.oclc.org/estimates.

Compant, S., Nowak, J., Coenye, T., Clément, C. \& Ait Barka, E. (2008). Diversity and occurrence of Burkholderia spp. in the natural environment. FEMS Microbiology Reviews 32, 607-626.

Cook, G. D. (2003). Fuel dynamics, nutrients and atmospheric chemistry. Fire in Tropical Savannas. (ed. by A. N. Andersen, G. D. Cook and R. J. Williams), pp. 47-58. Springer, NY.

Cooling, M., Hartley, S., Sim, D. A. \& Lester, P. J. (2011). The widespread collapse of an invasive species: Argentine ants (Linepithema humile) in New Zealand. Biology Letters 8, 430433. 
Corin, S. E., Abbott, K. L., Ritchie, P. A. \& Lester, P. J. (2007). Large scale unicoloniality: the population and colony structure of the invasive Argentine ant (Linepithema humile) in New Zealand. Insectes Sociaux 54, 275-282.

Cournault, L. \& Aron, S. (2009). Diploid males, diploid sperm production, and triploid females in the ant Tapinoma erraticum. Naturwissenschaften 19, 1393-1400.

Cox, M., Peterson, D. \& Biggs, P. (2010). SolexaQA: at-a-glance quality assessment of Illumina second-generation sequencing data. BMC Bioinformatics 11, 485.

Crawley, M. J. (1987). What makes a community invasible? Colonization, Succession and Stability. (ed. by A. J. Gray, M. J. Crawley and P. J. Edwards), pp. 429-453, Blackwell Scientific, Oxford, UK.

Cremer, S. \& Sixt, M. (2009). Analogies in the evolution of individual and social immunity. Philosophical Transactions of the Royal Society B: Biological Sciences 364, 129-142.

Cremer, S., Ugelvig, L. V., Drijfhout, F. P., Schlick-Steiner, B. C., Steiner, F. M., Seifert, B., Hughes, D. P., Schulz, A., Petersen, K. S., Konrad, H., Stauffer, C., Kiran, K., Espadaler, X., d'Ettorre, P., Aktaç, N., Eilenberg, J., Jones, G. R., Nash, D. R., Pedersen, J. S. \& Boomsma, J. J. (2008). The evolution of invasiveness in garden ants. PLoS ONE 3, e3838.

Crozier, R. H. (1971). Heterozygosity and sex determination in haplo-diploidy. American Naturalist 105, 399-412.

Crozier, R. H., Jermiin, L. S. \& Chiotis, M. (1997). Molecular evidence for a Jurassic origin of ants. Naturwissenschaften $\mathbf{8 4}, 22-23$.

Currie, C. R., Scott, J. A., Summerbell, R. C. \& Malloch, D. (1999). Fungus-growing ants use antibiotic-producing bacteria to control garden parasites. Nature 398, 701-704.

Dale, C. \& Moran, N. A. (2006). Molecular interactions between bacterial symbionts and their hosts. Cell 126, 453-465.

Dantas, G., Sommer, M. O. A., Oluwasegun, R. D. \& Church, G. M. (2008). Bacteria subsisting on antibiotics. Science 320, 100-103.

Davidson, D. W. (1997). The role of resource imbalances in the evolutionary ecology of tropical arboreal ants. Biological Journal of the Linnean Society 61, 153-181.

Davis, N. E., O'Dowd, D. J., Green, P. T. \& Mac Nally, R. (2008). Effects of an alien ant invasion on abundance, behavior, and reproductive success of endemic island birds. Conservation Biology 22, 1165-1176.

Davis, N. E., O'Dowd, D. J., Mac Nally, R. \& Green, P. T. (2010). Invasive ants disrupt frugivory by endemic island birds. Biology Letters 6, 85-88.

Dawkins, R. (1976). The Selfish Gene, Oxford University Press, Oxford, UK.

De Castro, F. \& Bolker, B. (2005). Mechanisms of disease-induced extinction. Ecology Letters 8 , $117-126$. 


\section{References}

de Souza, D., Bézier, A., Depoix, D., Drezen, J.-M. \& Lenoir, A. (2009). Blochmannia endosymbionts improve colony growth and immune defence in the ant Camponotus fellah. BMC Microbiology 9, 29.

Dillon, R. \& Charnley, K. (2002). Mutualism between the desert locust Schistocerca gregaria and its gut microbiota. Research in Microbiology 153, 503-509.

Dillon, R. J. \& Dillon, V. M. (2003). The gut bacteria of insects: nonpathogenic interactions. Annual Review of Entomology 49, 71-92.

Dlugosch, K. M. \& Parker, I. M. (2008). Founding events in species invasions: genetic variation, adaptive evolution, and the role of multiple introductions. Molecular Ecology 17, 431-449.

Dobata, S., Shimoji, H., Ohnishi, H., Hasegawa, E. \& Tsuji, K. (2012). Paternally inherited alleles in male body parts of an ant (Diacamma sp.) sex mosaic: implication for androgenetic male production in the Hymenoptera. Insectes Sociaux 59, 55-59.

Dray, S. \& Dufour, A. B. (2007). The ade4 package: implementing the duality diagram for ecologists. Journal of Statistical Software 22, 1-20.

Drescher, J. (2011). The ecology and population structure of the invasive yellow crazy ant Anoplolepis gracilipes, Doktorgrade (Doctor of Philosophy) dissertation. Julius-MaximilliansUniversität, Würzburg, Germany.

Drescher, J., Blüthgen, N. \& Feldhaar, H. (2007). Population structure and intraspecific aggression in the invasive ant species Anoplolepis gracilipes in Malaysian Borneo. Molecular Ecology 16, 1453-1465.

Drescher, J., Blüthgen, N., Schmitt, T., Bühler, J. \& Feldhaar, H. (2010). Societies drifting apart? Behavioural, genetic and chemical differentiation between supercolonies in the yellow crazy ant Anoplolepis gracilipes. PLoS ONE 5, e13581.

Drescher, J., Feldhaar, H. \& Blüthgen, N. (2011). Interspecific aggression and resource monopolization of the invasive ant Anoplolepis gracilipes in Malaysian Borneo. Biotropica 43, 93-99.

Duchesne, P. \& Turgeon, J. (2009). FLOCK: a method for quick mapping of admixture without source samples. Molecular Ecology Resources 9, 1333-1344.

Dybdahl, M. F. \& Drown, D. M. (2011). The absence of genotypic diversity in a successful parthenogenetic invader. Biological Invasions 13, 1663-1672.

Ehrlich, P. R. (1986). Which animal will invade? Ecology of Biological Invasions of North America and Hawaii. (ed. by H. A. Mooney and J. A. Drake), pp. 79-95, Springer, New York, NY.

Ellstrand, N. C. \& Schierenbeck, K. A. (2000). Hybridization as a stimulus for the evolution of invasiveness in plants? Proceedings of the National Academy of Sciences of the United States of America 97, 7043-7050.

Elton, C. S. (1958). The Ecology of Invasions by Animals and Plants, Methuen, London, UK. 
Engelstädter, J. \& Hurst, G. D. D. (2009). The ecology and evolution of microbes that manipulate host reproduction. Annual Review of Ecology, Evolution, and Systematics 40, 127-149.

Evanno, G., Regnaut, S. \& Goudet, J. (2005). Detecting the number of clusters of individuals using the software STRUCTURE: a simulation study. Molecular Ecology 14, 2611-2620.

Facon, B., Genton, B. J., Shykoff, J., Jarne, P., Estoup, A. \& David, P. (2006). A general ecoevolutionary framework for understanding bioinvasions. Trends in Ecology \& Evolution 21, $130-135$.

Facon, B., Pointier, J.-P., Jarne, P., Sarda, V. \& David, P. (2008). High genetic variance in lifehistory strategies within invasive populations by way of multiple introductions. Current Biology 18, 363-367.

Feare, C. (1999). Ants take over from rats on Bird Island, Seychelles. Bird Conservation International 9, 95-96.

Feldhaar, H. (2011). Bacterial symbionts as mediators of ecologically important traits of insect hosts. Ecological Entomology 36, 533-543.

Feldhaar, H., Drescher, J. \& Blüthgen, N. (2006). Characterization of microsatellite markers for the invasive ant species Anoplolepis gracilipes. Molecular Ecology Notes 6, 912-914.

Feldhaar, H., Foitzik, S. \& Heinze, J. (2008). Lifelong commitment to the wrong partner: hybridization in ants. Philosophical Transactions of the Royal Society B: Biological Sciences 363, 2891-2899.

Feldhaar, H. \& Gross, R. (2009). Insects as hosts for mutualistic bacteria. International Journal of Medical Microbiology 299, 1-8.

Feldhaar, H., Straka, J., Krischke, M., Berthold, K., Stoll, S., Mueller, M. \& Gross, R. (2007). Nutritional upgrading for omnivorous carpenter ants by the endosymbiont Blochmannia. BMC Biology 5, 48 .

Fisher, R. A. (1930). The Genetical Theory of Natural Selection, Clarendon Press, Oxford, UK.

Foitzik, S., Sturm, H., Pusch, K., D'Ettorre, P. \& Heinze, J. (2007). Nestmate recognition and intraspecific chemical and genetic variation in Temnothorax ants. Animal Behaviour 73, 9991007.

Foucaud, J., Fournier, D., Orivel, J., Delabie, J. H. C., Loiseau, A., Le Breton, J., Kergoat, G. J. \& Estoup, A. (2007). Sex and clonality in the little fire ant. Molecular Biology and Evolution 24, 2465-2473.

Foucaud, J., Jourdan, H., Le Breton, J., Loiseau, A., Konghouleux, D. \& Estoup, A. (2006). Rare sexual reproduction events in the clonal reproduction system of introduced populations of the little fire ant. Evolution 60, 1646-1657.

Foucaud, J., Orivel, J., Fournier, D., Delabie, J. H. C., Loiseau, A., Le Breton, J., Cerdan, P. \& Estoup, A. (2009). Reproductive system, social organization, human disturbance and ecological dominance in native populations of the little fire ant, Wasmannia auropunctata. Molecular Ecology 18, 5059-5073. 


\section{References}

Foucaud, J., Orivel, J., Loiseau, A., Delabie, J. H. C., Jourdan, H., Konghouleux, D., Vonshak, M., Tindo, M., Mercier, J. L., Fresneau, D., Mikissa, J. B., McGlynn, T., Mikheyev, A. S., Oettler, J. \& Estoup, A. (2010). Worldwide invasion by the little fire ant: routes of introduction and eco-evolutionary pathways. Evolutionary Applications 3, 363-374.

Fournier, D., De Biseau, J. C. \& Aron, S. (2009). Genetics, behaviour and chemical recognition of the invading ant Pheidole megacephala. Molecular Ecology 18, 186-199.

Fournier, D., Estoup, A., Orivel, J., Foucaud, J., Jourdan, H., Breton, J. L. \& Keller, L. (2005). Clonal reproduction by males and females in the little fire ant. Nature 435, 1230-1234.

Frankel, O. H. \& Soulé, M. E. (1981). Conservation and Evolution, Cambridge University Press, Cambridge, UK.

Frankham, R. (2005). Genetics and extinction. Biological Conservation 126, 131-140.

Gao, X. \& Starmer, J. (2008). AWclust: point-and-click software for non-parametric population structure analysis. BMC Bioinformatics 9, 77.

Gerlach, J. (2004). Impact of the invasive crazy ant Anoplolepis gracilipes on Bird Island, Seychelles. Journal of Insect Conservation 8, 15-25.

Gherna, R. L., Werren, J. H., Weisburg, W., Cote, R., Woese, C. R., Mandelco, L. \& Brenner, D. J. (1991). Arsenophonus nasoniae gen. nov., sp. nov., the causative agent of the son-killer trait in the parasitic wasp Nasonia vitripennis. International Journal of Systematic Bacteriology 41, 563-565.

Gilpin, M. E. \& Soulé, M. E. (1986). Minimum viable populations: processes of extinction. Conservation Biology: The Science of Scarcity and Diversity. (ed. by M. E. Soulé), pp. 19-34. Sinauer Associates, Sunderland, MA.

Giraud, T., Pedersen, J. S. \& Keller, L. (2002). Evolution of supercolonies: the Argentine ants of southern Europe. Proceedings of the National Academy of Sciences of the United States of America 99, 6075-6079.

Gladman, S. (2011). VelvetOptimiser v 2.2.0, Victorian Bioinformatics Consortium. Available from http://bioinformatics.net.au/software.velvetoptimiser.shtml.

Goodnight, C. J. (1987). On the effect of founder events on epistatic genetic variance. Evolution 41, 80-91.

Goodnight, C. J. (1988). Epistasis and the effect of founder events on the additive genetic variance. Evolution 42, 441-454.

Gosalbes, M. J., Latorre, A., Lamelas, A. \& Moya, A. (2010). Genomics of intracellular symbionts in insects. International Journal of Medical Microbiology 300, 271-278.

Gotelli, N. \& Entsminger, G. (2007). EcoSim: null models software for ecology. Version 7, available from http://garyentsminger.com/ecosim/index.htm.

Goudet, J. (2000). PCAGEN: a program to perform Principal Component Analysis (PCA) on gene frequency data, available from http://www2.unil.ch/popgen/softwares/pcagen.htm. 
Grapputo, A., Boman, S., Lindström, L., Lyytinen, A. \& Mappes, J. (2005). The voyage of an invasive species across continents: genetic diversity of North American and European Colorado potato beetle populations. Molecular Ecology 14, 4207-4219.

Green, P. T., Comport, S. \& Slip, D. (2004). The management and control of the invasive alien crazy ant (Anoplolepis gracilipes) on Christmas Island, Indian Ocean: the aerial baiting campaign September 2002), Unpublished final report to Environment Australia and the Crazy Ant Steering Committee, Monash University, Melbourne, Australia.

Green, P. T., O'Dowd, D. J. \& Lake, P. S. (1999). Alien ant invasion and ecosystem collapse on Christmas Island, Indian Ocean. Aliens 9, 2-4.

Greenslade, P. J. M. (1971). Interspecific competition and frequency changes among ants in Solomon Islands coconut plantations. Journal of Applied Ecology 8, 323-352.

Gueguen, G., Vavre, F., Gnankine, O., Peterschmitt, M., Charif, D., Chiel, E., Gottlieb, Y., Ghanim, M., Zchori-Fein, E. \& Fleury, F. (2010). Endosymbiont metacommunities, mtDNA diversity and the evolution of the Bemisia tabaci (Hemiptera: Aleyrodidae) species complex. Molecular Ecology 19, 4365-4376.

Guénard, B. \& Dunn, R. R. (2010). A new (old), invasive ant in the hardwood forests of eastern North America and its potentially widespread impacts. PLOS ONE 5, e11614.

Haines, I. H. \& Haines, J. B. (1978a). Colony structure, seasonality and food requirements of the crazy ant, Anoplolepis longipes (Jerd.), in the Seychelles. Ecological Entomology 3, 109-118.

Haines, I. H. \& Haines, J. B. (1978b). Pest status of the crazy ant, Anoplolepis longipes (Jerdon) (Hymenoptera: Formicidae), in the Seychelles. Bulletin of Entomological Research 68, 627638.

Haines, I. H., Haines, J. B. \& Cherrett, J. M. (1994). The impact and control of the crazy ant Anoplolepis longipes (Jerd.) in the Seychelles. Exotic Ants: Biology, Impact, and Control of Introduced Species. (ed. by D. F. Williams), pp. 206-218. Westview Press, Boulder, CO.

Hamilton, W. D. (1964). Genetical evolution of social behaviour, I and II. Journal of Theoretical Biology 7, 1-52.

Hartley, S., Krushelnycky, P. D. \& Lester, P. J. (2010). Integrating physiology, population dynamics and climate to make multi-scale predictions for the spread of an invasive insect: the Argentine ant at Haleakala National Park, Hawaii. Ecography 33, 83-94.

Hedges, L. M., Brownlie, J. C., O'Neill, S. L. \& Johnson, K. N. (2008). Wolbachia and virus protection in insects. Science 322, 702.

Hedrick, P. W. \& Parker, J. D. (1997). Evolutionary genetics and genetic variation of haplodiploids and x-linked genes. Annual Review of Ecology and Systematics 28, 55-83.

Hee, J. J., Holway, D. A., Suarez, A. V. \& Case, T. J. (2000). Role of propagule size in the success of incipient colonies of the invasive Argentine Ant. Conservation Biology 14, 559563.

Heinze, J. (2008). The demise of the standard ant. Myrmecological News 11, 9-20. 


\section{References}

Helanterä, H., Strassmann, J. E., Carrillo, J. \& Queller, D. C. (2009). Unicolonial ants: where do they come from, what are they and where are they going? Trends in Ecology \& Evolution 24, 341-349.

Heller, K. (2011). Tsetse flies rely on symbiotic Wigglesworthia for immune system development. PLoS Biol 9, e1001070.

Heller, N., Ingram, K. \& Gordon, D. (2008). Nest connectivity and colony structure in unicolonial Argentine ants. Insectes Sociaux 55, 397-403.

Helms Cahan, S., Parker, J. D., Rissing, S. W., Johnson, R. A., Polony, T. S., Weiser, M. D. \& Smith, D. R. (2002). Extreme genetic differences between queens and workers in hybridizing Pogonomyrmex harvester ants Proceedings of the Royal Society of London. Series B: Biological Sciences 269, 1871-1877.

Helms Cahan, S. \& Vinson, S. B. (2003). Reproductive division of labor between hybrid and nonhybrid offspring in a fire ant hybrid zone. Evolution 57, 1562-1570.

Helms, K. R. \& Vinson, S. B. (2002). Widespread association of the invasive ant Solenopsis invicta with an invasive mealybug. Ecology 83, 2425-2438.

Hierro, J. L., Maron, J. L. \& Callaway, R. M. (2005). A biogeographical approach to plant invasions: the importance of studying exotics in their introduced and native range. Journal of Ecology 93, 5-15.

Hill, M., Holm, K., Vel, T., Shah, N. J. \& Matyot, P. (2003). Impact of the introduced yellow crazy ant Anoplolepis gracilipes on Bird Island, Seychelles. Biodiversity and Conservation 12, 1969-1984.

Himler, A. G., Adachi-Hagimori, T., Bergen, J. E., Kozuch, A., Kelly, S. E., Tabashnik, B. E., Chiel, E., Duckworth, V. E., Dennehy, T. J., Zchori-Fein, E. \& Hunter, M. S. (2011). Rapid spread of a bacterial symbiont in an invasive whitefly is driven by fitness benefits and female bias. Science 332, 254-256.

Hoffmann, B. \& Parr, C. (2008). An invasion revisited: the African big-headed ant (Pheidole megacephala) in northern Australia. Biological Invasions 10, 1171-1181.

Hoffmann, B. \& Saul, W.-C. (2010). Yellow crazy ant (Anoplolepis gracilipes) invasions within undisturbed mainland Australian habitats: no support for biotic resistance hypothesis. Biological Invasions 12, 3093-3108.

Hölldobler, B. \& Wilson, E. O. (1990). The Ants, The Belknap Press of Harvard University Press, Cambridge, MA.

Holway, D. A. (1999). Competitive mechanisms underlying the displacement of native ants by the invasive Argentine ant. Ecology 80, 238.

Holway, D. A., Lach, L., Suarez, A. V., Tsutsui, N. D. \& Case, T. J. (2002). The causes and consequences of ant invasions. Annual Review of Ecology and Systematics 33, 181-233.

Holway, D. A., Suarez, A. V. \& Case, T. J. (1998). Loss of intraspecific aggression in the success of a widespread invasive social insect. Science 282, 949-952. 
Hoover, K., Grove, M., Gardner, M., Hughes, D. P., McNeil, J. \& Slavicek, J. (2011). A gene for an extended phenotype. Science 333, 1401.

Hothorn, T., Hornik, K., van de Wiel, M. A. \& Zeileis, A. (2008). Implementing a class of permutation tests: the coin package. Journal of Statistical Software 28, 1-23.

Human, K. G. \& Gordon, D. M. (1999). Behavioral interactions of the invasive Argentine ant with native ant species. Insectes Sociaux 46, 159-163.

Hurlbert, S. H. (1971). The nonconcept of species diversity: a critique and alternative parameters. Ecology 52, 577-586.

Huson, D. H., Mitra, S., Ruscheweyh, H.-J., Weber, N. \& Schuster, S. C. (2011). Integrative analysis of environmental sequences using MEGAN4. Genome Research 21, 1552-1560.

Ihaka, R. \& Gentleman, R. (1996). R: a language for data analysis and graphics. Journal of Computational and Graphical Statistics 5, 299-314.

Illumina (2011). Genome Analyzer IIx System specification sheet: Illumina sequencing, Illumina Inc., San Diego, $\quad$ CA. available from http://www.illumina.com/documents/products/datasheets/datasheet genome analyzerIIx.pdf.

Ishak, H., Plowes, R., Sen, R., Kellner, K., Meyer, E., Estrada, D., Dowd, S. \& Mueller, U. (2011). Bacterial diversity in Solenopsis invicta and Solenopsis geminata ant colonies characterized by 16S amplicon 454 pyrosequencing. Microbial Ecology 61, 821-831.

Janssen, P. H. (2006). Identifying the dominant soil bacterial taxa in libraries of $16 \mathrm{~S}$ rRNA and 16S rRNA genes. Appl. Envir. Microbiol. 72, 1719-1728.

Jones, S. R. \& Phillips, S. A. J. (1985). Gynandromorphism in the ant Pheidole dentata Mayr (Hymenoptera, Formicidae). Proceedings of the Entomological Society Washington 87, 583586.

Jost, L. (2006). Entropy and diversity. Oikos 113, 363-375.

Kalinowski, S. T. (2005). HP-RARE: a computer program for performing rarefaction on measures of allelic diversity. Molecular Ecology Notes 5, 187-189.

Keller, L. (1995). Parasites, worker polymorphism, and queen number in social insects. American Naturalist 145, 842-847.

Keller, L. (2007). Uncovering the biodiversity of genetic and reproductive systems: time for a more open approach. American Naturalist 169, 1-8.

Keller, L., Liautard, C., Reuter, M., Brown, W. D., Sundstrom, L. \& Chapuisat, M. (2001). Sex ratio and Wolbachia infection in the ant Formica exsecta. Heredity 87, 227-233.

Keller, L. \& Ross, K. G. (1998). Selfish genes: a green beard in the red fire ant. Nature 394, 573575.

Keller, L. F. \& Waller, D. M. (2002). Inbreeding effects in wild populations. Trends in Ecology \& Evolution 17, 230-241. 


\section{References}

Kellner, K. \& Heinze, J. (2010). Mechanism of facultative parthenogenesis in the ant Platythyrea punctata. Evolutionary Ecology 25, 77-89.

Kersters, K., Vos, P., Gillis, M., Swings, J., Vandamme, P. \& Stackebrandt, E. (2006). Introduction to the proteobacteria. The Prokaryotes. (ed. by M. Dworkin, S. Falkow, E. Rosenberg, K.-H. Schleifer and E. Stackebrandt), pp. 3-37. Springer, New York, NY.

Kikuchi, Y., Meng, X.-Y. \& Fukatsu, T. (2005). Gut symbiotic bacteria of the genus Burkholderia in the broad-headed bugs Riptortus clavatus and Leptocorisa chinensis (Heteroptera: Alydidae). Applied and Environmental Microbiology 71, 4035-4043.

Kirschenbaum, R. \& Grace, J. K. (2008). Agonistic interactions among invasive ant species (Hymenoptera: Formicidae) from two habitats on Oahu, Hawaii. Sociobiology 51, 543-553.

Kobayashi, K., Hasegawa, E. \& Ohkawara, K. (2008). Clonal reproduction by males of the ant Vollenhovia emeryi (Wheeler). Entomological Science 11, 167-172.

Kolbe, J. J., Glor, R. E., Rodriguez Schettino, L., Chamizo Lara, A., Larson, A. \& Losos, J. B. (2004). Genetic variation increases during biological invasion by a Cuban lizard. Nature 431, $177-181$.

Koskinen, M. T., Hirvonen, H., Landry, P.-A. \& Primmer, C. R. (2004). The benefits of increasing the number of microsatellites utilized in genetic population studies: an empirical perspective. Hereditas 141, 61-67.

Krieger, M. J. B. \& Keller, L. (2000). Mating frequency and genetic structure of the Argentine ant Linepithema humile. Molecular Ecology 9, 119-126.

Krushelnycky, P. D. \& Gillespie, R. G. (2008). Compositional and functional stability of arthropod communities in the face of ant invasions. Ecological Applications 18, 1547-1562.

Krushelnycky, P. D., Holway, D. A. \& LeBrun, E. G. (2010). Invasion processes and causes of success. Ant Ecology. (ed. by L. Lach, C. L. Parr and K. L. Abbott), pp. 245-260. Oxford University Press, Oxford, UK.

Krushelnycky, P. D., Joe, S. M., Medeiros, A. C., Daehler, C. C. \& Loope, L. L. (2005). The role of abiotic conditions in shaping the long-term patterns of a high-elevation Argentine ant invasion. Diversity and Distributions 11, 319-331.

Kunin, V., Copeland, A., Lapidus, A., Mavromatis, K. \& Hugenholtz, P. (2008). A bioinformatician's guide to metagenomics. Microbiology and Molecular Biology Reviews 72, 557-578.

Lach, L. (2005). Interference and exploitation competition of three nectar-thieving invasive ant species. Insectes Sociaux 52, 257-262.

Lach, L. \& Hoffmann, B. D. (2011). Are invasive ants better plant-defense mutualists? A comparison of foliage patrolling and herbivory in sites with invasive yellow crazy ants and native weaver ants. Oikos 120, 9-16.

Lande, R. (1980). Genetic variation and phenotypic evolution during allopatric speciation. The American Naturalist 116, 463-479. 
Lande, R. (1988). Genetics and demography in biological conservation. Science 241, 1455-1460.

Le Breton, J., Jourdan, H., Chazeau, J., Orivel, J. \& Dejean, A. (2005). Niche opportunity and ant invasion: the case of Wasmannia auropunctata in a New Caledonian rain forest. Journal of Tropical Ecology 21, 93-98.

LeBrun, E. G., Tillberg, C. V., Suarez, A. V., Folgarait, P. J., Smith, C. R. \& Holway, D. A. (2007). An experimental study of competition between red imported fire ants and Argentine ants in their native range. Ecology 88, 63-75.

Lee, C. E. (2002). Evolutionary genetics of invasive species. Trends in Ecology and Evolution 17, 386-391.

Lefèvre, T., Williams, A. J. \& de Roode, J. C. (2011). Genetic variation in resistance, but not tolerance, to a protozoan parasite in the monarch butterfly. Proceedings of the Royal Society of London. Series B: Biological Sciences 278, 751-759.

Legendre, P. \& Fortin, M. J. (2010). Comparison of the Mantel test and alternative approaches for detecting complex multivariate relationships in the spatial analysis of genetic data. Molecular Ecology Resources 10, 831-844.

Lester, P., Abbott, K., Sarty, M. \& Burns, K. (2009). Competitive assembly of South Pacific invasive ant communities. BMC Ecology $9,3$.

Lester, P. J. \& Burns, K. C. (2008). Terrestrial arthropods. Encyclopedia of Ecology. (ed. by S. E. Jørgensen and B. D. Fath), pp. 3536-3543, Elsevier, Oxford, UK.

Lester, P. J. \& Tavite, A. (2004). Long-legged ants, Anoplolepis gracilipes (Hymenoptera: Formicidae), have invaded Tokelau, changing composition and dynamics of ant and invertebrate communities. Pacific Science 58, 391-401.

Leston, D. (1973). The ant mosaic - tropical tree crops and the limiting of pests and diseases. Tropical Pest Management 19, 311-341.

Lewis, T., Cherrett, J. M., Haines, I., J.B., H. \& Mathias, P. L. (1976). The crazy ant Anoplolepis longipes (Jerd.) (Hymenoptera: Formicidae) in the Seychelles, and its chemical control. Bulletin of Entomological Research 66, 97-111.

Lewontin, R. C. (1965). Selection for colonizing ability. The Genetics of Colonizing Species. (ed. by H. G. Baker and G. L. Stebbins), pp. 77-94. Academic Press, New York, NY.

Li, R., Yu, C., Li, Y., Lam, T.-W., Yiu, S.-M., Kristiansen, K. \& Wang, J. (2009). SOAP2: An improved ultrafast tool for short read alignment. Bioinformatics 25, 1966-1967.

Li, S.-C., Chan, W.-C., Lai, C.-H., Tsai, K.-W., Hsu, C.-N., Jou, Y.-S., Chen, H.-C., Chen, C.-H. \& Lin, W.-c. (2011). UMARS: Un-MAppable Reads Solution. BMC Bioinformatics 12, S9.

Liebert, A. E., Sumana, A. \& Starks, P. T. (2005). Diploid males and their triploid offspring in the paper wasp Polistes dominulus. Biology Letters 1, 200-203.

Liersch, S. \& Schmid-Hempel, P. (1998). Genetic variation within social insect colonies reduces parasite load. Proceedings of the Royal Society of London. Series B: Biological Sciences 265, 221-225. 


\section{References}

Liu, J., Dong, M., Miao, S., Li, Z., Song, M. \& Wang, R. (2006). Invasive alien plants in China: role of clonality and geographical origin. Biological Invasions 8, 1461-1470.

Lowe, S., Browne, M. \& Boudjelas, S. (2000). 100 of the world's worst invasive alien species - a selection from the Global Invasive Species database, The Invasive Species Specialist Group (ISSG) of the Species Survival Commission (SSC) of the World Conservation Union (IUCN), Gland, Switzerland.

Luquet, E., Garner, T. W. J., Léna, J.-P., Bruel, C., Joly, P., Lengagne, T., Grolet, O. \& Plénet, S. (2012). Genetic erosion in wild populations makes resistance to a pathogen more costly. Evolution in press.

Lynch, M. \& Hill, W. G. (1986). Phenotypic evolution by neutral mutation. Evolution 40, 915935.

Mack, R. N., Simberloff, D., Lonsdale, W. M., Evans, H., Clout, M. \& Bazzaz, F. A. (2000). Biotic invasions: causes, epidemiology, global consequences, and control. Ecological Applications 10, 689-710.

Macom, T. E. \& Porter, S. D. (1996). Comparison of polygyne and monogyne red imported fire ant (Hymenoptera: Formicidae) population densities. Annals of the Entomological Society of America 89, 535-543.

Madden, T. (2002). The BLAST Sequence Analysis Tool. The NCBI Handbook. (ed. by J. McEntyre and J. Ostell). National Center for Biotechnology Information, Bethesda, MD.

Majer, J. D. (1984). Recolonisation by ants in rehabilitated open-cut mines in northern Australia. Reclamation and Revegetation Research 2, 279-298.

Martin, S. J., Helanterä, H. \& Drijfhout, F. P. (2011). Is parasite pressure a driver of chemical cue diversity in ants? Proceedings of the Royal Society of London. Series B: Biological Sciences 278, 496-503.

Matsui, S., Kikuchi, T., Akatani, K., Horie, S. \& Takagi, M. (2009). Harmful effects of invasive yellow crazy ant Anoplolepis gracilipes on three land bird species of Minami-Daito Island. Ornithological Science 8, 81-86.

Mattila, H. R. \& Seeley, T. D. (2007). Genetic diversity in honey bee colonies enhances productivity and fitness. Science 317, 362-364.

Mayr, E. (1942). Systematics and the Origin of Species, New York, Columbia University Press.

Mayr, E. (1963). Animal Species and Evolutuon, Harvard University Press, Cambridge, MA.

Mayr, E. (1965). The nature of colonization in birds. The Genetics of Colonizing Species. (ed. by H. G. Baker and G. L. Stebbins), pp. 29-47. Academic Press, New York, NY.

Mazerolle, M. J. (2011). AICcmodavg: model selection and multimodel inference based on $(Q) A I C(c) . \quad R$ package, version 1.21, available at http://CRAN.Rproject. org/package $=$ AICcmodavg. 
McKenna, M. (1973). Sweepstakes, filters, corridors, Noah's Arks, and Beached Viking Funeral Ships in palaeogeography. Implications of Continental Drift to the Earth Sciences. (ed. by D. H. Tarling and S. K. Runcorn), pp. 295-308. Academic Press, New York, NY.

McNatty, A., Abbott, K. \& Lester, P. (2009). Invasive ants compete with and modify the trophic ecology of hermit crabs on tropical islands. Oecologia 160, 187-194.

Menke, S. B. \& Holway, D. A. (2006). Abiotic factors control invasion by Argentine ants at the community scale. Journal of Animal Ecology 75, 368-376.

Merçot, H. \& Poinsot, D. (2009). Infection by Wolbachia: from passengers to residents. Comptes Rendus Biologies 332, 284-297.

Mergeay, J., Verschuren, D. \& Meester, L. D. (2006). Invasion of an asexual American water flea clone throughout Africa and rapid displacement of a native sibling species. Proceedings: Biological Sciences 273, 2839-2844.

Mitchell, C. E. \& Power, A. G. (2003). Release of invasive plants from fungal and viral pathogens. Nature 421, 625-627.

Mittler, T. E. (1971). Some effects on the aphid Myzus persicae of ingesting antibiotics incorporated into artificial diets. Journal of Insect Physiology 17, 1333-1347.

Moles, A. T., Gruber, M. A. M. \& Bonser, S. P. (2008). A new framework for predicting invasive plant species. Journal of Ecology 96, 13-17.

Moran, N. A., Degnan, P. H., Santos, S. R., Dunbar, H. E. \& Ochman, H. (2005). The players in a mutualistic symbiosis: insects, bacteria, viruses, and virulence genes. Proceedings of the National Academy of Sciences of the United States of America 102, 16919-16926.

Moreau, C. S. (2009). Inferring ant evolution in the age of molecular data (Hymenoptera: Formicidae). Myrmecological News 12, 201-210.

Moreau, C. S., Bell, C. D., Vila, R., Archibald, S. B. \& Pierce, N. E. (2006). Phylogeny of the ants: diversification in the age of angiosperms. Science 312, 101-104.

Morin, P. A., Luikart, G., Wayne, R. K. \& the, S. N. P. w. g. (2004). SNPs in ecology, evolution and conservation. Trends in Ecology \& Evolution 19, 208-216.

Morrison, L. W. (2000). Mechanisms of interspecific competition among an invasive and two native fire ants. Oikos 90, 238-252.

Morrison, L. W. (2002). Long-term impacts of an arthropod-community invasion by the imported fire ant, Solenopsis invicta. Ecology 83, 2337-2345.

Mueller-Dombois, D. \& Fosberg, F. R. (1998). Vegetation of the Tropical Pacific Islands, Springer-Verlag, New York, NY.

Munoz-Torres, M. C., Reese, J. T., Childers, C. P., Bennett, A. K., Sundaram, J. P., Childs, K. L., Anzola, J. M., Milshina, N. \& Elsik, C. G. (2011). Hymenoptera Genome Database: integrated community resources for insect species of the order Hymenoptera. Nucleic Acids Research 39, D658-D662. 


\section{References}

Nanodrop (2005). ND-1000 Spectrophotometer: V3.2 User's Manual, Nanodrop Technologies Inc., Wilmington, DE.

Nei, M., Maruyama, T. \& Chakraborty, R. (1975). The bottleneck effect and genetic variability in populations. Evolution 29, 1-10.

Nei, M. \& Roychoudhury, A. K. (1974). Sampling variances of heterozygosity and genetic distance. Genetics 76, 379-390.

Neville, P., O'Dowd, D. \& Yen, A. (2008). Issues and implications for research on disturbed oceanic islands illustrated through an ant survey of the Cocos (Keeling) Islands. Journal of Insect Conservation 12, 313-323.

Nuwantha Ariyaratne, P. \& Sung, W.-K. (2011). PE-Assembler: de novo assembler using short paired-end reads. Bioinformatics 27, 167-174.

O'Brien, S. J. \& Evermann, J. F. (1988). Interactive influence of infectious disease and genetic diversity in natural populations. Trends in Ecology and Evolution 3, 254-259.

O'Dowd, D. J., Green, P. T. \& Lake, P. S. (1999). Status, impact, and recommendations for research and management of exotic invasive ants in Christmas Island National Park, Environment Australia, Darwin, Australia.

O'Dowd, D. J., Green, P. T. \& Lake, P. S. (2003). Invasional 'meltdown' on an oceanic island. Ecology Letters 6, 812-817.

Ohkawara, K., Nakayama, M., Satoh, A., Trindl, A. \& Heinze, J. (2006). Clonal reproduction and genetic caste differences in a queen-polymorphic ant, Vollenhovia emeryi. Biology Letters 2 , 359-363.

Oi, D. H., Williams, D. F., Pereira, R. M., Horton, P. M., Davis, T. S., Hyder, A. H., Bolton, H. T., Zeichner, B. C., Porter, S. D., Hoch, L. A., Boswell, M. L. \& Williams, G. (2008). Combining biological and chemical controls for the management of red imported fire ants (Hymenoptera: Formicidae). American Entomologist 54, 46-55.

Oldroyd, B. P. \& Fewell, J. H. (2007). Genetic diversity promotes homeostasis in insect colonies. Trends in Ecology \&amp; Evolution 22, 408-413.

Oliver, K. M., Degnan, P. H., Burke, G. R. \& Moran, N. A. (2010). Facultative symbionts in aphids and the horizontal transfer of ecologically important traits. Annual Review of Entomology 55, 247-266.

Oliver, T. H., Pettitt, T., Leather, S. R. \& Cook, J. M. (2008). Numerical abundance of invasive ants and monopolisation of exudate-producing resources - a chicken and egg situation. Insect Conservation and Diversity 1, 208-214.

Omarjee, J., Balandreau, J., Spaull, V. W. \& Cadet, P. (2008). Relationships between Burkholderia populations and plant parasitic nematodes in sugarcane. Applied Soil Ecology 39, $1-14$.

Pachepsky, E. \& Levine, J. M. (2010). Density dependence slows invader spread in fragmented landscapes. The American Naturalist 177, 18-28. 
Pamilo, P. (1991). Evolution of colony characteristics in social insects. II. Number of reproductive individuals. The American Naturalist 138, 412-433.

Peakall, R. \& Smouse, P. E. (2006). GenAlEx 6: Genetic analysis in Excel. Population genetic software for teaching and research. Molecular Ecology Notes 6, 288-295.

Pearcy, M., Aron, S., Doums, C. \& Keller, L. (2004). Conditional use of sex and parthenogenesis for worker and queen production in ants. Science 306, 1780-1783.

Pearcy, M., Goodisman, M. A. D. \& Keller, L. (2011). Sib mating without inbreeding in the longhorn crazy ant. Proceedings of the Royal Society of London. Series B: Biological Sciences 278, 2677-2681.

Pearcy, M., Hardy, O. \& Aron, S. (2006). Thelytokous parthenogenesis and its consequences on inbreeding in an ant. Heredity 96, 377-382.

Pedersen, J. S., Krieger, M. J. B., Vogel, V., Giraud, T. \& Keller, L. (2006). Native supercolonies of unrelated individuals in the invasive Argentine ant. Evolution 60, 782-791.

Peeters, C. (1987). The reproductive division of labour in the queenless ponerine ant Rhytidoponera sp. 12. Insectes Sociaux 34, 75-86.

Perring, T. M. (2001). The Bemisia tabaci species complex. Crop Protection 20, 725-737.

Petit, R. J. (2004). Biological invasions at the gene level. Diversity and Distributions 10, 159-165.

Petrosino, J. F., Highlander, S., Luna, R. A., Gibbs, R. A. \& Versalovic, J. (2009). Metagenomic pyrosequencing and microbial identification. Clinical Chemistry 55, 856-866.

Pfeiffer, M., Cheng Tuck, H. \& Chong Lay, T. (2008). Exploring arboreal ant community composition and co-occurrence patterns in plantations of oil palm Elaeis guineensis in Borneo and Peninsular Malaysia. Ecography 31, 21-32.

Piertney, S. B. \& Oliver, M. K. (2005). The evolutionary ecology of the major histocompatibility complex. Heredity 96, 7-21.

Pimentel, D. (2005). Environmental consequences and economic costs of alien species. Invasive Plants: Ecological and Agricultural Aspects. (ed. by Inderjit), pp. 269-276. Birkhäuser Verlag, Basel, Switzerland.

Porter, S. D. \& Savignano, D. A. (1990). Invasion of polygyne fire ants decimates native ants and disrupts arthropod community. Ecology 71, 2095-2106.

Porter, S. D., Williams, D. F., Patterson, R. S. \& Fowler, H. G. (1997). Intercontinental differences in the abundance of Solenopsis fire ants (Hymenoptera: Formicidae): Escape from natural enemies? Environmental Entomology 26, 373-384.

Pritchard, J. K., Stephens, M. \& Donnelly, P. (2000). Inference of population structure using multilocus genotype data. Genetics 155, 945-959.

Pulliam, H. R. (1988). Sources, sinks, and population regulation. The American Naturalist 132, 652-661. 


\section{References}

Quek, S.-P., Davies, S. J., Itino, T. \& Pierce, N. E. (2004). Codiversification in an ant-plant mutualism: stem texture and the evolution of host use in Crematogaster (Formicidae: Myrmicinae) inhabitants of Macaranga (Euphorbiaceae). Evolution 58, 554-570.

R Development Core Team (2011). $R$ : a language and environment for statistical computing, $\mathrm{R}$ Foundation for Statistical Computing, Vienna, Austria. ISBN 3-900051-07-0, URL http://www.R-project.org/.

Rao, N. S. \& Veeresh, G. K. (1991). Some observations on the biology and behaviour of crazy ant, Anoplolepis longipes (Jerdon) (Hymenoptera : Formicidae). Entomon 16, 261-267.

Raymond, M. \& Rousset, F. (1995). GENEPOP (version 1.2): population genetics software for exact tests and ecumenicism. Journal of Heredity 86, 248-249.

Reber, A., Castella, G., Christe, P. \& Chapuisat, M. (2008). Experimentally increased group diversity improves disease resistance in an ant species. Ecology Letters 11, 682-689.

Ren, M. X., Zhang, Q. G. \& Zhang, D. Y. (2005). Random amplified polymorphic DNA markers reveal low genetic variation and a single dominant genotype in Eichhornia crassipes populations throughout China. Weed Research 45, 236-244.

Rice, W. R. (1989). Analyzing tables of statistical tests. Evolution 43, 223-225.

Rioux Paquette, S. (2011). PopGenKit: useful functions for (batch) file conversion and data resampling in microsatellite datasets, $\mathrm{R}$ package, version 1.0. Available at http://cran.rproject.org/web/packages/PopGenKit/index.html.

Rodovalho, C., Ferro, M., Fonseca, F., Antonio, E., Guilherme, I., Henrique-Silva, F. \& Bacci, M. (2011). Expressed sequence tags from Atta laevigata and identification of candidate genes for the control of pest leaf-cutting ants. BMC Research Notes 4, 203.

Roman, J. \& Darling, J. A. (2007). Paradox lost: genetic diversity and the success of aquatic invasions. Trends in Ecology \& Evolution 22, 454-464.

Rosenthal, R. (1991). Meta-analytic Procedures for Social Research, Sage, Newbury Park, CA.

Ross, K. G., Krieger, M. J. B. \& Shoemaker, D. D. (2003). Alternative genetic foundations for a key social polymorphism in fire ants. Genetics 165, 1853-1867.

Ross, K. G. \& Shoemaker, D. D. (2008). Estimation of the number of founders of an invasive pest insect population: the fire ant Solenopsis invicta in the USA. Proceedings of the Royal Society of London. Series B: Biological Sciences 275, 2231-2240.

Ross, K. G., Vargo, E. L. \& Keller, L. (1996). Social evolution in a new environment: the case of introduced fire ants. Proceedings of the National Academy of Sciences of the United States of America 93, 3021-3025.

Rosset, H., Keller, L. \& Chapuisat, M. (2005). Experimental manipulation of colony genetic diversity had no effect on short-term task efficiency in the Argentine ant Linepithema humile. Behavioral Ecology and Sociobiology 58, 87-98.

Roulston, T. H., Buczkowski, G. \& Silverman, J. (2003). Nestmate discrimination in ants: effect of bioassay on aggressive behavior. Insectes Sociaux 50, 151-159. 
Rousset, F. (2008). GENEPOP'007: a complete re-implementation of the GENEPOP software for Windows and Linux. Molecular Ecology Resources 8, 103-106.

Rowles, A. \& O’Dowd, D. (2007). Interference competition by Argentine ants displaces native ants: implications for biotic resistance to invasion. Biological Invasions 9, 73-85.

Russell-Smith, J., Murphy, B. P., Meyer, M. C. P., Cook, G. D., Maier, S., Edwards, A. C., Schatz, J. \& Brocklehurst, P. (2009). Improving estimates of savanna burning emissions for greenhouse accounting in northern Australia. International Journal of Wildland Fire 18, 1-18.

Russell, J. A. \& Moran, N. A. (2005). Horizontal transfer of bacterial symbionts: heritability and fitness effects in a novel aphid host. Applied and Environmental Microbiology 71, 7987-7994.

Russell, J. A., Moreau, C. S., Goldman-Huertas, B., Fujiwara, M., Lohman, D. J. \& Pierce, N. E. (2009). Bacterial gut symbionts are tightly linked with the evolution of herbivory in ants. Proceedings of the National Academy of Sciences of the United States of America 106, 2123621241.

Sachs, J. L. \& Simms, E. L. (2006). Pathways to mutualism breakdown. Trends in Ecology \&amp; Evolution 21, 585-592.

Sagata, K. \& Lester, P. J. (2009). Behavioural plasticity associated with propagule size, resources, and the invasion success of the Argentine ant Linepithema humile. Journal of Applied Ecology 46, 19-27.

Sakai, A. K., Allendorf, F. W., Holt, J. S., Lodge, D. M., Molofsky, J., With, K. A., Baughman, S., Cabin, R. J., Cohen, J. E., Ellstrand, N. C., McCauley, D. E., O’Neil, P., Parker, I. M., Thompson, J. N. \& Weller, S. G. (2001). The population biology of invasive species. Annual Review of Ecology, Evolution and Systematics 32, 305-332.

Saltonstall, K. (2002). Cryptic invasion by a non-native genotype of the common reed, Phragmites australis, into North America. Proceedings of the National Academy of Sciences of the United States of America 99, 2445-2449.

Sanders, N., Gotelli, N. J., Heller, N. \& Gordon, D. M. (2003). Community disassembly by an invasive species. Proceedings of the National Academy of Sciences of the United States of America 100, 2474-2477.

Sanders, N. J. \& Suarez, A. V. (2011). Elton's insights into the ecology of ant invasions: Lessons learned and lessons still to be learned. Fifty Years of Invasion Ecology: The Legacy of Charles Elton. (ed. by D. M. Richardson), pp. 239-251. Blackwell Publishing Ltd, Oxford, UK.

Santos, A. V., Dillon, R. J., Dillon, V. M., Reynolds, S. E. \& Samuels, R. I. (2004). Ocurrence of the antibiotic producing bacterium Burkholderia sp. in colonies of the leaf-cutting ant Atta sexdens rubropilosa. FEMS Microbiology Letters 239, 319-323.

Sarty, M., Abbott, K. \& Lester, P. (2007). Community level impacts of an ant invader and food mediated coexistence. Insectes Sociaux 54, 166 - 173.

Savage, A. M., Rudgers, J. A. \& Whitney, K. D. (2009). Elevated dominance of extrafloral nectary-bearing plants is associated with increased abundances of an invasive ant and reduced native ant richness. Diversity and Distributions 15, 751-761. 


\section{References}

Sax, D. F. \& Brown, J. H. (2000). The paradox of invasion. Global Ecology and Biogeography 9 , 363-371.

Schlüns, H. \& Crozier, R. H. (2009). Molecular and chemical immune defences in ants (Hymenoptera: Formicidae). Myrmecological News 12, 237-249.

Schmid-Hempel, P. (1998). Parasites in Social Insects, Princeton University Press, Princeton, NJ.

Schmid-Hempel, P. \& Crozier, R. H. (1999). Polyandry versus polygyny versus parasites. Philosophical Transactions: Biological Sciences 354, 507-515.

Schmid-Hempel, P., Schmid-Hempel, R., Brunner, P. C., Seeman, O. D. \& Allen, G. R. (2007). Invasion success of the bumblebee, Bombus terrestris, despite a drastic genetic bottleneck. Heredity 99, 414-422.

Schuelke, M. (2000). An economic method for the fluorescent labeling of PCR fragments. Nature 18, 233-234.

Schwander, T., Cahan, S. H. \& Keller, L. (2006). Genetic caste determination in Pogonomyrmex harvester ants imposes costs during colony founding. Journal of Evolutionary Biology 19, 402409 .

Schwander, T., Lo, N., Beekman, M., Oldroyd, B. P. \& Keller, L. (2010). Nature versus nurture in social insect caste differentiation. Trends in Ecology \& Evolution 25, 275-282.

Scortese, C. R. (1997). PALEOMAP Paleogeographic Atlas, PALEOMAP Progress Report \#90, Department of Geology, University of Texas at Arlington.

Sebastien, A., Gruber, M. \& Lester, P. (2012). Prevalence and genetic diversity of three bacterial endosymbionts (Wolbachia, Arsenophonus, and Rhizobiales) associated with the invasive yellow crazy ant (Anoplolepis gracilipes). Insectes Sociaux 59, 33-40.

Sepp, R., Szabo, I., Uda, H. \& Sakamoto, H. (1994). Rapid techniques for DNA extraction from routinely processed archival tissue for use in PCR. Journal of Clinical Pathology 47, 318-323.

Sherman, P. W., Seeley, T. D. \& Reeve, H. K. (1988). Parasites, pathogens, and polyandry in social Hymenoptera. American Naturalist 131, 602-610.

Shoemaker, D. D., Ross, K. G., Keller, L., Vargo, E. L. \& Werren, J. H. (2000). Wolbachia infections in native and introduced populations of fire ants (Solenopsis spp.). Insect Molecular Biology 9, 661-673.

Shykoff, J. A. \& Schmid-Hempel, P. (1991). Parasites and the advantage of genetic variability within social insect colonies. Proceedings of the Royal Society of London. Series B: Biological Sciences 243, 55-58.

Silverman, J. \& Liang, D. (2001). Colony disassociation following diet partitioning in a unicolonial ant. Naturwissenschaften 88, 73-77.

Simberloff, D. (2009). The role of propagule pressure in biological invasions. Annual Review of Ecology, Evolution, and Systematics 40, 81-102. 
Simberloff, D. \& Gibbons, L. (2004). Now you see them, now you don't! - Population crashes of established introduced species. Biological Invasions 6, 161-172.

Simon, J.-C., Delmotte, F., Rispe, C. \& Crease, T. (2003). Phylogenetic relationships between parthenogens and their sexual relatives: the possible routes to parthenogenesis in animals. Biological Journal of the Linnean Society 79, 151-163.

Simpson, J. T., Wong, K., Jackman, S. D., Schein, J. E., Jones, S. J. M. \& Birol, I. (2009). ABySS: a parallel assembler for short read sequence data. Genome Research 19, 1117-1123.

Slatkin, M. (1993). Isolation by distance in equilibrium and non-equilibrium populations. Evolution 47, 264-279.

Smith, C. D., Zimin, A., Holt, C., Abouheif, E., Benton, R., Cash, E., Croset, V., Currie, C. R., Elhaik, E., Elsik, C. G., Favé, M.-J., Fernandes, V., Gadau, J., Gibson, J. D., Graur, D., Grubbs, K. J., Hagen, D. E., Helmkampf, M., Holley, J.-A., Hu, H., Viniegra, A. S. I., Johnson, B. R., Johnson, R. M., Khila, A., Kim, J. W., Laird, J., Mathis, K. A., Moeller, J. A., Muñoz-Torres, M. C., Murphy, M. C., Nakamura, R., Nigam, S., Overson, R. P., Placek, J. E., Rajakumar, R., Reese, J. T., Robertson, H. M., Smith, C. R., Suarez, A. V., Suen, G., Suhr, E. L., Tao, S., Torres, C. W., van Wilgenburg, E., Viljakainen, L., Walden, K. K. O., Wild, A. L., Yandell, M., Yorke, J. A. \& Tsutsui, N. D. (2011a). Draft genome of the globally widespread and invasive Argentine ant (Linepithema humile). Proceedings of the National Academy of Sciences of the United States of America 108, 5673-5678.

Smith, C. R., Smith, C. D., Robertson, H. M., Helmkampf, M., Zimin, A., Yandell, M., Holt, C., Hu, H., Abouheif, E., Benton, R., Cash, E., Croset, V., Currie, C. R., Elhaik, E., Elsik, C. G., Favé, M.-J., Fernandes, V., Gibson, J. D., Graur, D., Gronenberg, W., Grubbs, K. J., Hagen, D. E., Viniegra, A. S. I., Johnson, B. R., Johnson, R. M., Khila, A., Kim, J. W., Mathis, K. A., Muñoz-Torres, M. C., Murphy, M. C., Mustard, J. A., Nakamura, R., Niehuis, O., Nigam, S., Overson, R. P., Placek, J. E., Rajakumar, R., Reese, J. T., Suen, G., Tao, S., Torres, C. W., Tsutsui, N. D., Viljakainen, L., Wolschin, F. \& Gadau, J. (2011b). Draft genome of the red harvester ant Pogonomyrmex barbatus. Proceedings of the National Academy of Sciences of the United States of America 108, 5667-5672.

Steiner, F. M., Schlick-Steiner, B. C., Moder, K., Stauffer, C., Arthofer, W., Buschinger, A., Espadaler, X., Christian, E., Einfinger, K., Lorbeer, E., Schafellner, C., Ayasse, M. \& Crozier, R. H. (2007). Abandoning aggression but maintaining self-nonself discrimination as a first stage in ant supercolony formation Current Biology 17, 1903-1907.

Stoll, S., Gadau, J., Gross, R. O. Y. \& Feldhaar, H. (2007). Bacterial microbiota associated with ants of the genus Tetraponera. Biological Journal of the Linnean Society 90, 399-412.

Stouthamer, R., Breeuwer, J. A. J., Luck, R. F. \& Werren, J. H. (1993). Molecular identification of microorganisms associated with parthenogenesis. Nature 361, 66-68.

Strayer, D. L., Eviner, V. T., Jeschke, J. M. \& Pace, M. L. (2006). Understanding the long-term effects of species invasions. Trends in Ecology and Evolution 21, 645-651.

Suárez-Moreno, Z., Caballero-Mellado, J., Coutinho, B., Mendonça-Previato, L., James, E. \& Venturi, V. (2012). Common features of environmental and potentially beneficial plantassociated Burkholderia. Microbial Ecology 63, 249-266. 


\section{References}

Suarez, A. V., Holway, D. A., Liang, D., Tsutsui, N. D. \& Case, T. J. (2002). Spatiotemporal patterns of intraspecific aggression in the invasive Argentine ant. Animal Behaviour 64, 697708.

Suarez, A. V., Holway, D. A. \& Tsutsui, N. D. (2008). Genetics and behavior of a colonizing species: the invasive Argentine ant. The American Naturalist 172, S72-S84.

Suarez, A. V. \& Tsutsui, N. D. (2008). The evolutionary consequences of biological invasions. Molecular Ecology 17, 351-360.

Suarez, A. V., Tsutsui, N. D., Holway, D. A. \& Case, T. J. (1999). Behavioral and genetic differentiation between native and introduced populations of the Argentine ant. Biological Invasions 1, 43-53.

Suen, G., Teiling, C., Li, L., Holt, C., Abouheif, E., Bornberg-Bauer, E., Bouffard, P., Caldera, E. J., Cash, E., Cavanaugh, A., Denas, O., Elhaik, E., Favé, M.-J., Gadau, J., Gibson, J. D., Graur, D., Grubbs, K. J., Hagen, D. E., Harkins, T. T., Helmkampf, M., Hu, H., Johnson, B. R., Kim, J., Marsh, S. E., Moeller, J. A., Muñoz-Torres, M. C., Murphy, M. C., Naughton, M. C., Nigam, S., Overson, R., Rajakumar, R., Reese, J. T., Scott, J. J., Smith, C. R., Tao, S., Tsutsui, N. D., Viljakainen, L., Wissler, L., Yandell, M. D., Zimmer, F., Taylor, J., Slater, S. C., Clifton, S. W., Warren, W. C., Elsik, C. G., Smith, C. D., Weinstock, G. M., Gerardo, N. M. \& Currie, C. R. (2011). The genome sequence of the leaf-cutter ant Atta cephalotes reveals insights into its obligate symbiotic lifestyle. PLoS Genetics 7, e1002007.

Suhr, E. L., McKechnie, S. W. \& O'Dowd, D. J. (2009). Genetic and behavioural evidence for a city-wide supercolony of the invasive Argentine ant Linepithema humile (Mayr) (Hymenoptera: Formicidae) in southeastern Australia. Australian Journal of Entomology 48, 79-83.

Suomalainen, E., Saura, A. \& Lokki, J. (1987). Cytology and Evolution in Parthenogenesis, CRC Press Inc., Boca Raton, FL.

Suwabe, M., Ohnishi, H., Kikuchi, T., Kawara, K. \& Tsuji, K. (2009). Difference in seasonal activity pattern between non-native and native ants in subtropical forest of Okinawa Island, Japan. Ecological Research 24, 637-643.

Tamaki, H., Wright, C. L., Li, X., Lin, Q., Hwang, C., Wang, S., Thimmapuram, J., Kamagata, Y. \& Liu, W.-T. (2011). Analysis of 16S rRNA amplicon sequencing options on the Roche/454 next-generation Titanium sequencing platform. PLOS ONE 6, e25263.

Tamura, K., Dudley, J., Nei, M. \& Kumar, S. (2007). MEGA4: Molecular Evolutionary Genetics Analysis (MEGA) software version 4.0. Molecular Biology and Evolution 24, 1596-1599.

Tanaka, H., Ohnishi, H., Tatsuta, H. \& Tsuji, K. (2011). An analysis of mutualistic interactions between exotic ants and honeydew producers in the Yanbaru district of Okinawa Island, Japan. Ecological Research 26, 931-941.

Tarpy, D. R. (2003). Genetic diversity within honeybee colonies prevents severe infections and promotes colony growth. Proceedings of the Royal Society of London. Series B: Biological Sciences 270, 99-103.

Taylor, C. M. \& Hastings, A. (2005). Allee effects in biological invasions. Ecology Letters 8, 895-908. 
Teixeira, L., Ferreira, Á. \& Ashburner, M. (2008). The bacterial symbiont Wolbachia induces resistance to RNA viral infections in Drosophila melanogaster. PLoS Biology 6, e1000002.

Templeton, A. R. (1980). The theory of speciation via the founder principle. Genetics 94, 10111038.

Thao, M. L. \& Baumann, P. (2004). Evidence for multiple acquisition of Arsenophonus by whitefly species (Sternorrhyncha: Aleyrodidae). Current Microbiology 48, 140-144.

Thierry, M., Becker, N., Hajri, A., Reynaud, B., Lett, J. M. \& Delatte, H. (2011). Symbiont diversity and non-random hybridization among indigenous (Ms) and invasive (B) biotypes of Bemisia tabaci. Molecular Ecology 20, 2172-2187.

Thomas, M., Becker, K., Abbott, K. \& Feldhaar, H. (2010). Supercolony mosaics: two different invasions by the yellow crazy ant, Anoplolepis gracilipes, on Christmas Island, Indian Ocean. Biological Invasions 12, 677-687.

Thompson, J. D., Higgins, D. G. \& Gibson, T. J. (1994). CLUSTAL W: improving the sensitivity of progressive multiple sequence alignment through sequence weighting, position-specific gap penalties and weight matrix choice. Nucleic Acids Research 22, 4673-4680.

Tillberg, C. V., Holway, D. A., LeBrun, E. G. \& Suarez, A. V. (2007). Trophic ecology of invasive Argentine ants in their native and introduced ranges. Proceedings of the National Academy of Sciences of the United States of America 104, 20856-20861.

Tobin, P. C., Berec, L. \& Liebhold, A. M. (2011). Exploiting Allee effects for managing biological invasions. Ecology Letters 14, 615-624.

Torchin, M. E., Lafferty, K. D., Dobson, A. P., McKenzie, V. J. \& Kuris, A. M. (2003). Introduced species and their missing parasites. Nature 421, 628-630.

Tsuchida, T., Koga, R., Horikawa, M., Tsunoda, T., Maoka, T., Matsumoto, S., Simon, J.-C. \& Fukatsu, T. (2010). Symbiotic bacterium modifies aphid body color. Science 330, 1102-1104.

Tsutsui, N. D. \& Case, T. J. (2001). Population genetics and colony structure of the Argentine ant (Linepithema humile) in its native and introduced ranges. Evolution 55, 976-985.

Tsutsui, N. D., Kauppinen, S. N., Oyafuso, A. F. \& Grosberg, R. K. (2003a). The distribution and evolutionary history of Wolbachia infection in native and introduced populations of the invasive argentine ant (Linepithema humile). Molecular Ecology 12, 3057-3068.

Tsutsui, N. D., Suarez, A. V. \& Grosberg, R. K. (2003b). Genetic diversity, asymmetrical aggression, and recognition in a widespread invasive species. Proceedings of the National Academy of Sciences of the United States of America 100, 1078-1083.

Tsutsui, N. D., Suarez, A. V., Holway, D. A. \& Case, T. J. (2000). Reduced genetic variation and the success of an invasive species. Proceedings of the National Academy of Sciences of the United States of America 97, 5948-5953.

Turelli, M. \& Hoffmann, A. A. (1995). Cytoplasmic incompatibility in Drosophila simulans: dynamics and parameter estimates from natural populations. Genetics 140, 1319-1338. 


\section{References}

Ugelvig, L. V., Kronauer, D. J. C., Schrempf, A., Heinze, J. \& Cremer, S. (2010). Rapid antipathogen response in ant societies relies on high genetic diversity. Proceedings of the Royal Society B: Biological Sciences 277, 2821-2828.

Väli, Ü., Einarsson, A., Waits, L. \& Ellegren, H. (2008). To what extent do microsatellite markers reflect genome-wide genetic diversity in natural populations? Molecular Ecology 17, 38083817.

Valles, S. M., Oi, D. H. \& Porter, S. D. (2010). Seasonal variation and the co-occurrence of four pathogens and a group of parasites among monogyne and polygyne fire ant colonies. Biological Control 54, 342-348.

Valvano, M. A., Keith, K. E. \& Cardona, S. T. (2005). Survival and persistence of opportunistic Burkholderia species in host cells. Current Opinion in Microbiology 8, 99-105.

van Borm, S., Buschinger, A., Boomsma, J. J. \& Billen, J. (2002). Tetraponera ants have gut symbionts related to nitrogen-fixing root-nodule bacteria. Proceedings of the Royal Society of London. Series B: Biological Sciences 269, 2023-2027.

van Heerwaarden, B., Willi, Y., Kristensen, T. N. \& Hoffmann, A. A. (2008). Population bottlenecks increase additive genetic variance but do not break a selection limit in rain forest Drosophila. Genetics 179, 2135-2146.

van Oosterhout, C., Hutchinson, W. F., Willis, D. P. M. \& Shipley, P. (2004). MICROCHECKER: software for identifying and correcting genotyping errors in microsatellite data. Molecular Ecology Notes 4, 535-538.

van Wilgenburg, E., Driessen, G. \& Beukeboom, L. W. (2006). Single locus complementary sex determination in Hymenoptera: an "unintelligent" design? Frontiers in Zoology 3.

van Wilgenburg, E., Torres, C. W. \& Tsutsui, N. D. (2010). The global expansion of a single ant supercolony. Evolutionary Applications 3, 136-143.

Vandamme, P., Govan, J. \& LiPuma, J. (2007a). Diversity and role of Burkholderia spp. Burkholderia: Molecular Microbiology and Genomics. (ed. by T. Coenye and P. Vandamme), pp. 1-28. Horizon Bioscience, Norfolk, UK.

Vandamme, P., Opelt, K., Knöchel, N., Berg, C., Schönmann, S., De Brandt, E., Eberl, L., Falsen, E. \& Berg, G. (2007b). Burkholderia bryophila sp. nov. and Burkholderia megapolitana sp. nov., moss-associated species with antifungal and plant-growth-promoting properties. International Journal of Systematic and Evolutionary Microbiology 57, 2228-2235.

Vander Meer, R. \& Morel, L. (1998). Nestmate recognition in ants. Pheromone Communication in Social Insects: Ants, Wasps, Bees, and Termites. (ed. by R. Vander Meer, M. Breed, M. Winston and K. Espilie), pp. 79-103. Westview Press, Boulder, CO.

Viljakainen, L. \& Pamilo, P. (2008). Selection on an antimicrobial peptide defensin in ants. Journal of Molecular Evolution 67, 643-652.

Vogel, V., Pedersen, J. S., Giraud, T., Krieger, M. J. B. \& Keller, L. (2010). The worldwide expansion of the Argentine ant. Diversity and Distributions 16, 170-186. 
Volny, V. P. \& Gordon, D. M. (2002). Genetic basis for queen-worker dimorphism in a social insect. Proceedings of the National Academy of Sciences of the United States of America 99, 6108-6111.

Von Holle, B. \& Simberloff, D. (2005). Ecological resistance to biological invasion overwhelmed by propagule pressure. Ecology 86, 3212-3218.

Wang, J. (2002). An estimator for pairwise relatedness using molecular markers. Genetics 160, 1203-1215.

Ward, D. \& Beggs, J. (2007). Coexistence, habitat patterns and the assembly of ant communities in the Yasawa islands, Fiji. Acta Oecologica 32, 215-223.

Warwick, R. M., Clarke, K. R. \& Suharsono, S. (1990). A statistical analysis of coral community responses to the 1982-83 El Niño in the Thousand Islands, Indonesia. Coral Reefs 8, 171-179.

Wenseleers \& Billen (2000). No evidence for Wolbachia-induced parthenogenesis in the social Hymenoptera. Journal of Evolutionary Biology 13, 277-280.

Wenseleers, T., Ito, F., Van Borm, S., Huybrechts, R., Volckaert, F. \& Billen, J. (1998). Widespread occurrence of the microorganism Wolbachia in ants. Proceedings of the Royal Society of London. Series B: Biological Sciences 265, 1447-1452.

Wenseleers, T., Sundström, L. \& Billen, J. (2002). Deleterious Wolbachia in the ant Formica truncorum. Proceedings of the Royal Society of London. Series B: Biological Sciences 269, 623-629.

Wetterer, J. K. (2005). Worldwide distribution and potential spread of the long-legged ant, Anoplolepis gracilipes (Hymenoptera: Formicidae). Sociobiology 45, 77-97.

Wilder, S. M., Holway, D. A., Suarez, A. V., LeBrun, E. G. \& Eubanks, M. D. (2011). Intercontinental differences in resource use reveal the importance of mutualisms in fire ant invasions. Proceedings of the National Academy of Sciences 108, 20639-20644.

Willi, Y., Van Buskirk, J. \& Hoffmann, A. A. (2006). Limits to the adaptive potential of small populations. Annual Review of Ecology, Evolution, and Systematics 37, 433-458.

Wilson, E. O. (1963). Social modifications related to rareness in ant species. Evolution 17, 249253.

Wilson, E. O. (1987). Causes of ecological success: the case of the ants. The Journal of Animal Ecology 56, 1-9.

Wilson, J. R. U., Dormontt, E. E., Prentis, P. J., Lowe, A. J. \& Richardson, D. M. (2009a). Biogeographic concepts define invasion biology. Trends in Ecology \& Evolution 24, 586-586.

Wilson, J. R. U., Dormontt, E. E., Prentis, P. J., Lowe, A. J. \& Richardson, D. M. (2009b). Something in the way you move: dispersal pathways affect invasion success. Trends in Ecology \& Evolution 24, 136-144.

Wood, S. N. (2006). Generalized Additive Models: an introduction with R. Chapman \& Hall / CRC Texts in Statistical Science. (ed. by B. P. Carlin, C. Chatfield, M. Tanner and T. Zidek). Taylor \& Francis Group, Boca Raton, FL. 


\section{References}

Wright, S. (1931). Evolution in Mendelian populations. Genetics 16, 97-159.

Wurm, Y., Wang, J., Riba-Grognuz, O., Corona, M., Nygaard, S., Hunt, B. G., Ingram, K. K., Falquet, L., Nipitwattanaphon, M., Gotzek, D., Dijkstra, M. B., Oettler, J., Comtesse, F., Shih, C.-J., Wu, W.-J., Yang, C.-C., Thomas, J., Beaudoing, E., Pradervand, S., Flegel, V., Cook, E. D., Fabbretti, R., Stockinger, H., Long, L., Farmerie, W. G., Oakey, J., Boomsma, J. J., Pamilo, P., Yi, S. V., Heinze, J., Goodisman, M. A. D., Farinelli, L., Harshman, K., Hulo, N., Cerutti, L., Xenarios, I., Shoemaker, D. \& Keller, L. (2011). The genome of the fire ant Solenopsis invicta. Proceedings of the National Academy of Sciences 108, 5679-5684.

Yang, C.-C., Ascunce, M. S., Luo, L.-Z., Shao, J.-G., Shih, C.-J. \& Shoemaker, D. (2012). Propagule pressure and colony social organization are associated with the successful invasion and rapid range expansion of fire ants in China. Molecular Ecology 21, 817-833.

Yanoviak, S. P., Kaspari, M., Dudley, R. \& Poinar Jr., G. (2008). Parasite-induced fruit mimicry in a tropical canopy ant. The American Naturalist 171, 536-544.

Yoshizawa, J., Mimori, K., Yamauchi, K. \& Tsuchida, K. (2009). Sex mosaics in a male dimorphic ant Cardiocondyla kagutsuchi. Naturwissenschaften 96, 49-55.

Young, G. R., Bellis, G. A., Brown, G. R. \& Smith, E. S. C. (2001). The crazy ant Anoplolepis gracilipes (Smith) (Hymenoptera: Formicidae) in east Arnhem Land, Australia. Australian Entomologist 28, 97-104.

Zayed, A., Constantin, Ş. A. \& Packer, L. (2007). Successful biological invasion despite a severe genetic load. PLoS ONE 2, e868.

Zayed, A. \& Packer, L. (2005). Complementary sex determination substantially increases extinction proneness of haplodiploid populations. Proceedings of the National Academy of Sciences of the United States of America 102, 10742-10746.

Zerbino, D. \& Birney, E. (2008). Velvet: algorithms for de novo short read assembly using de Bruijn graphs. Genome Research 18, 821 - 829.

Zerbino, D. R. (2010). Using the Velvet de novo assembler for short-read sequencing technologies. Current Protocols in Bioinformatics. (ed. by A. D. Baxevanis), pp. 11.15.11-12. John Wiley \& Sons, Inc., Online Library.

Zhang, Z., Schwartz, S., Wagner, L. \& Miller, W. (2000). A greedy algorithm for aligning DNA sequences. Journal of Computational Biology 7, 203-214.

Zientz, E., Feldhaar, H., Stoll, S. \& Gross, R. (2005). Insights into the microbial world associated with ants. Archives of Microbiology 184, 199-206. 


\section{Appendices}

\section{Supplementary figures}

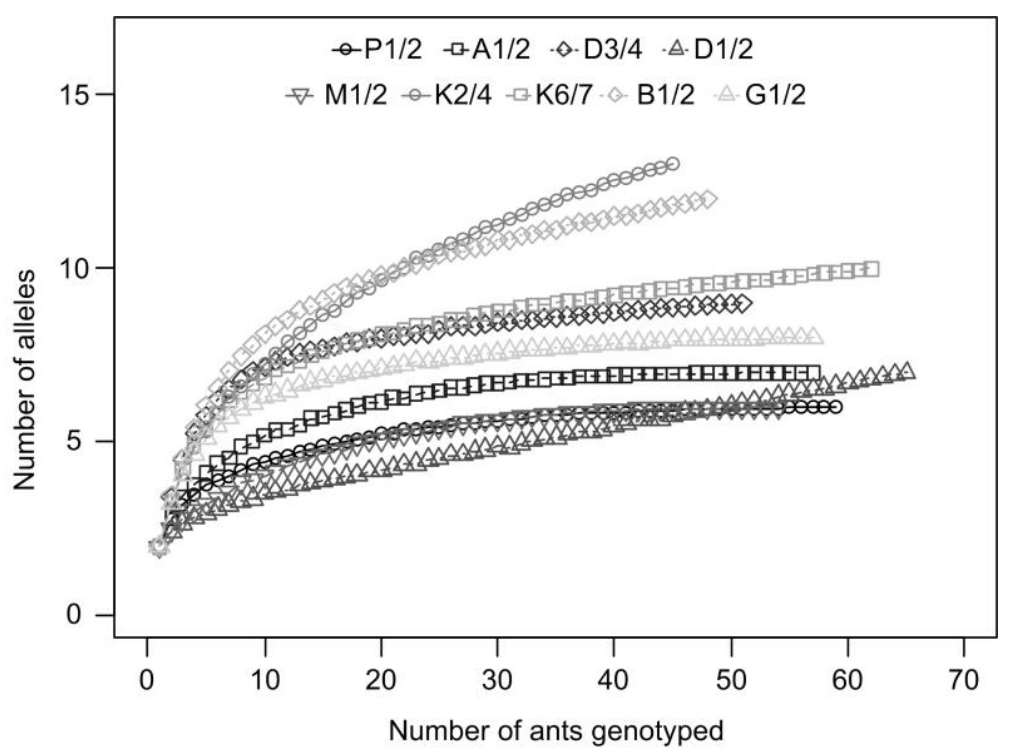

Supplementary Fig. 4.1: Allele discovery curves for the most polymorphic locus (Ano8) for all nine Anoplolepis gracilipes invaded sites (18 plots) in the study, based on the complete dataset of 539 ants. Where curves have not flattened completely there were no further samples for genotyping. 


\section{Appendices}
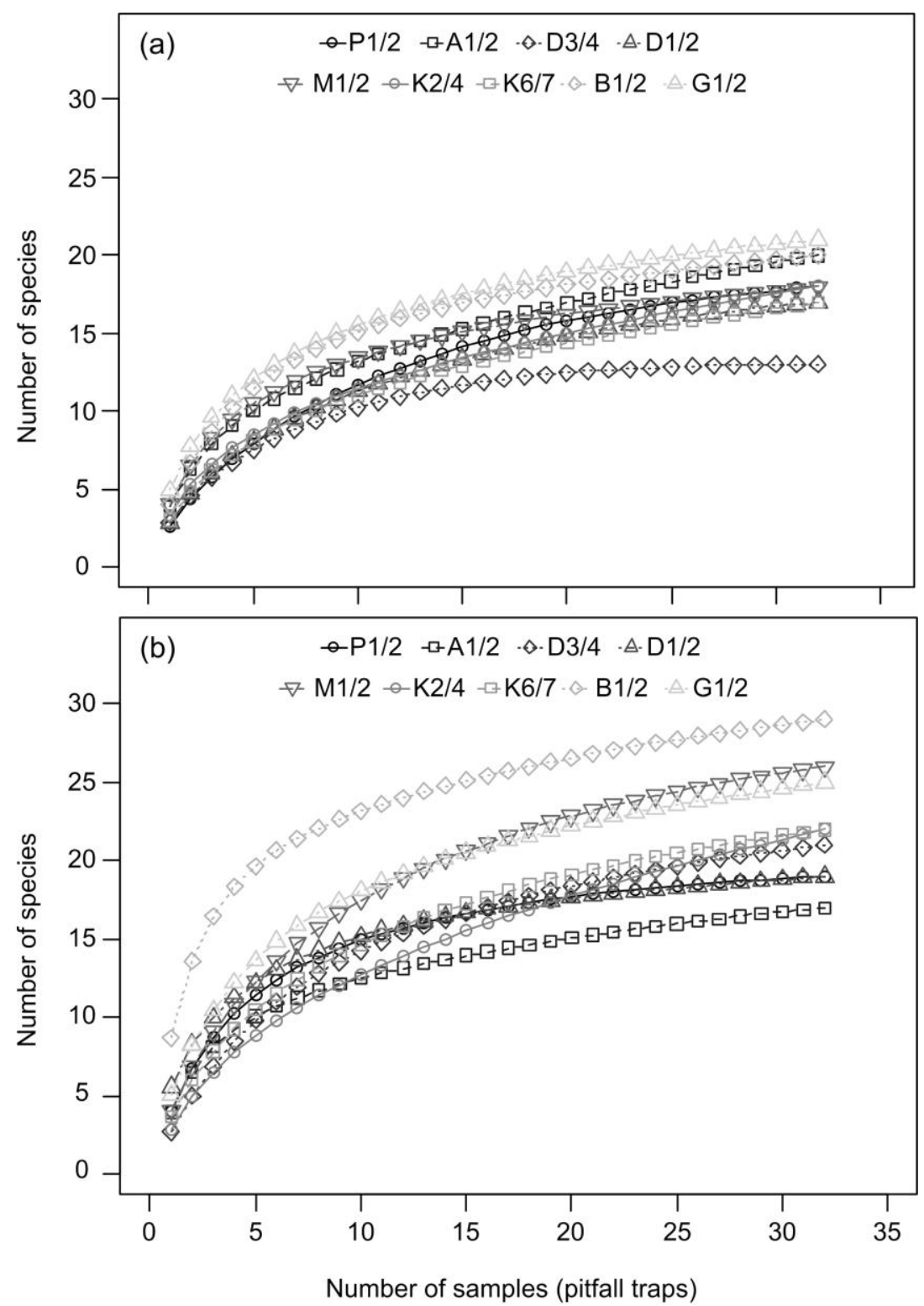

Supplementary Fig. 4.2: Species accumulation curves based on pitfall trap catches for Anoplolepis gracilipes: (a) invaded; and (b) uninvaded sites in Arnhem Land, Australia. 


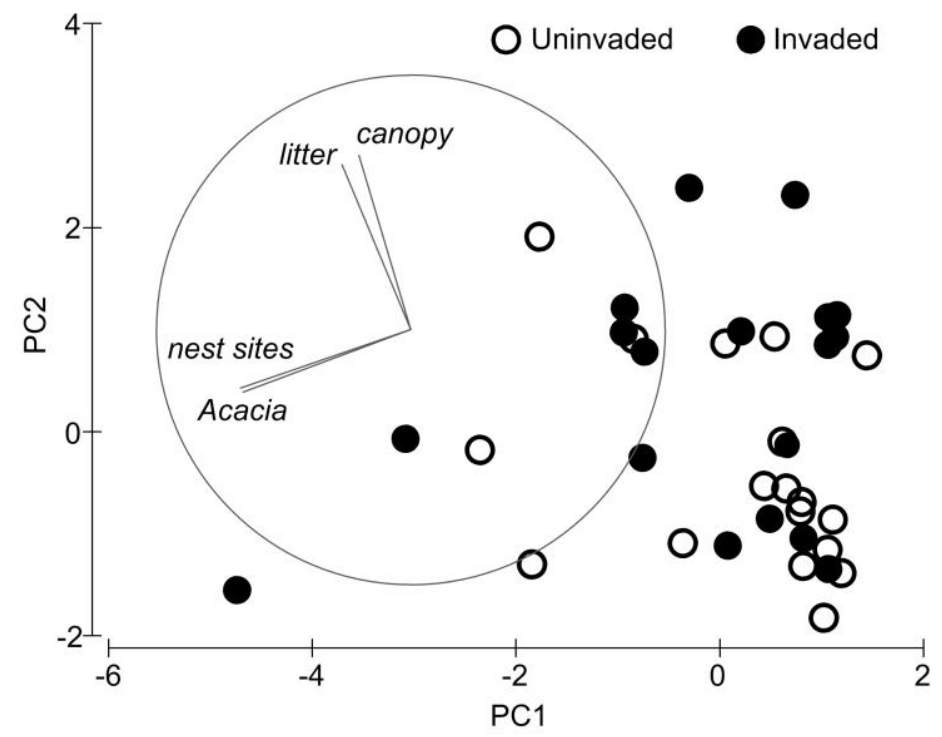

Supplementary Fig. 4.3: Principal component analysis of Anoplolepis gracilipes invaded and uninvaded plots based on Euclidean distance of standardised values for the availability of nest sites, canopy cover, presence of Acacia spp. and leaf litter depth. 


\section{Appendices}

\section{Supplementary tables}

Supplementary Table 2.1: Allele frequencies and sample sizes for Chapter 2.

\begin{tabular}{|c|c|c|c|c|c|}
\hline Locus & Allele & Queens & Sperm & Males & Workers \\
\hline \multirow[t]{3}{*}{ Anol } & $\mathbf{N}$ & 39 & 46 & 29 & 310 \\
\hline & 97 & 0.000 & 0.402 & 0.259 & 0.498 \\
\hline & 101 & 1.000 & 0.598 & 0.741 & 0.502 \\
\hline \multirow[t]{5}{*}{ Ano3 } & $\mathbf{N}$ & 47 & 46 & 29 & 310 \\
\hline & 162 & 0.883 & 0.663 & 0.724 & 0.502 \\
\hline & 174 & 0.000 & 0.000 & 0.000 & 0.011 \\
\hline & 176 & 0.117 & 0.337 & 0.276 & 0.444 \\
\hline & 178 & 0.000 & 0.000 & 0.000 & 0.044 \\
\hline \multirow[t]{3}{*}{ Ano4 } & $\mathbf{N}$ & 47 & 45 & 29 & 310 \\
\hline & 156 & 1.000 & 0.644 & 0.828 & 0.502 \\
\hline & 174 & 0.000 & 0.356 & 0.172 & 0.498 \\
\hline \multirow[t]{7}{*}{ Ano7 } & $\mathbf{N}$ & 39 & 45 & 29 & 310 \\
\hline & 228 & 0.013 & 0.000 & 0.000 & 0.000 \\
\hline & 230 & 0.000 & 0.478 & 0.293 & 0.505 \\
\hline & 240 & 0.000 & 0.033 & 0.000 & 0.005 \\
\hline & 242 & 0.936 & 0.467 & 0.690 & 0.263 \\
\hline & 244 & 0.051 & 0.022 & 0.017 & 0.219 \\
\hline & 246 & 0.000 & 0.000 & 0.000 & 0.008 \\
\hline \multirow[t]{17}{*}{ Ano8 } & $\mathbf{N}$ & 45 & 46 & 29 & 310 \\
\hline & 208 & 0.000 & 0.043 & 0.000 & 0.000 \\
\hline & 210 & 0.000 & 0.011 & 0.000 & 0.015 \\
\hline & 212 & 0.444 & 0.293 & 0.034 & 0.156 \\
\hline & 214 & 0.300 & 0.185 & 0.000 & 0.108 \\
\hline & 216 & 0.222 & 0.130 & 0.862 & 0.210 \\
\hline & 218 & 0.000 & 0.022 & 0.000 & 0.016 \\
\hline & 262 & 0.000 & 0.000 & 0.000 & 0.024 \\
\hline & 264 & 0.000 & 0.000 & 0.000 & 0.019 \\
\hline & 266 & 0.000 & 0.000 & 0.000 & 0.045 \\
\hline & 268 & 0.000 & 0.033 & 0.000 & 0.042 \\
\hline & 270 & 0.000 & 0.000 & 0.000 & 0.006 \\
\hline & 272 & 0.022 & 0.196 & 0.000 & 0.090 \\
\hline & 274 & 0.011 & 0.065 & 0.086 & 0.232 \\
\hline & 276 & 0.000 & 0.000 & 0.017 & 0.026 \\
\hline & 278 & 0.000 & 0.000 & 0.000 & 0.002 \\
\hline & 282 & 0.000 & 0.022 & 0.000 & 0.008 \\
\hline
\end{tabular}


Supplementary Table 3.1 (a): Allele frequencies and sample sizes for Chapter 3, for loci with $>2$ alleles only (loci with two alleles had 0.500:0.500 allele frequencies). Samples A1 - D2.

\begin{tabular}{|c|c|c|c|c|c|c|c|}
\hline \multirow[b]{2}{*}{ Locus } & \multirow[b]{2}{*}{ Allele } & \multicolumn{6}{|c|}{ Sample } \\
\hline & & A1 & A2 & B1 & B2 & D1 & D2 \\
\hline \multirow[t]{6}{*}{ Ano3 } & $\mathbf{N}$ & 31 & 26 & 23 & 25 & 33 & 32 \\
\hline & 162 & 0.500 & 0.500 & 0.478 & 0.500 & 0.500 & 0.500 \\
\hline & 164 & 0.000 & 0.000 & 0.022 & 0.000 & 0.000 & 0.000 \\
\hline & 174 & 0.000 & 0.000 & 0.000 & 0.000 & 0.030 & 0.000 \\
\hline & 176 & 0.500 & 0.500 & 0.500 & 0.500 & 0.455 & 0.297 \\
\hline & 178 & 0.000 & 0.000 & 0.000 & 0.000 & 0.015 & 0.203 \\
\hline \multirow[t]{8}{*}{ Ano 7} & $\mathbf{N}$ & 31 & 26 & 23 & 25 & 33 & 32 \\
\hline & 230 & 0.500 & 0.500 & 0.500 & 0.480 & 0.500 & 0.500 \\
\hline & 232 & 0.000 & 0.000 & 0.000 & 0.020 & 0.000 & 0.000 \\
\hline & 240 & 0.000 & 0.000 & 0.000 & 0.000 & 0.015 & 0.016 \\
\hline & 242 & 0.000 & 0.000 & 0.000 & 0.080 & 0.273 & 0.078 \\
\hline & 244 & 0.500 & 0.500 & 0.478 & 0.400 & 0.212 & 0.406 \\
\hline & 246 & 0.000 & 0.000 & 0.022 & 0.020 & 0.000 & 0.000 \\
\hline & 248 & 0.000 & 0.000 & 0.000 & 0.000 & 0.000 & 0.000 \\
\hline \multirow[t]{19}{*}{ Ano8 } & $\mathbf{N}$ & 31 & 26 & 23 & 25 & 33 & 32 \\
\hline & 208 & 0.000 & 0.000 & 0.000 & 0.020 & 0.000 & 0.000 \\
\hline & 210 & 0.000 & 0.000 & 0.087 & 0.020 & 0.000 & 0.000 \\
\hline & 212 & 0.000 & 0.000 & 0.239 & 0.240 & 0.000 & 0.000 \\
\hline & 214 & 0.048 & 0.000 & 0.130 & 0.200 & 0.015 & 0.000 \\
\hline & 216 & 0.403 & 0.500 & 0.043 & 0.020 & 0.470 & 0.500 \\
\hline & 218 & 0.048 & 0.000 & 0.000 & 0.000 & 0.015 & 0.000 \\
\hline & 254 & 0.000 & 0.000 & 0.000 & 0.000 & 0.000 & 0.000 \\
\hline & 256 & 0.000 & 0.000 & 0.000 & 0.000 & 0.000 & 0.000 \\
\hline & 264 & 0.000 & 0.000 & 0.000 & 0.000 & 0.000 & 0.000 \\
\hline & 266 & 0.000 & 0.000 & 0.000 & 0.000 & 0.000 & 0.000 \\
\hline & 268 & 0.000 & 0.000 & 0.022 & 0.040 & 0.000 & 0.000 \\
\hline & 270 & 0.032 & 0.019 & 0.043 & 0.160 & 0.000 & 0.000 \\
\hline & 272 & 0.097 & 0.096 & 0.130 & 0.100 & 0.000 & 0.016 \\
\hline & 274 & 0.355 & 0.173 & 0.130 & 0.160 & 0.394 & 0.391 \\
\hline & 276 & 0.016 & 0.212 & 0.174 & 0.000 & 0.106 & 0.078 \\
\hline & 278 & 0.000 & 0.000 & 0.000 & 0.020 & 0.000 & 0.000 \\
\hline & 282 & 0.000 & 0.000 & 0.000 & 0.000 & 0.000 & 0.016 \\
\hline & 284 & 0.000 & 0.000 & 0.000 & 0.020 & 0.000 & 0.000 \\
\hline
\end{tabular}


Supplementary Table 3.1 (b): Allele frequencies and sample sizes for Chapter 3, for loci with $>2$ alleles only (loci with two alleles had 0.500:0.500 allele frequencies). Samples D3 - K4.

\begin{tabular}{|c|c|c|c|c|c|c|c|}
\hline \multirow[b]{2}{*}{ Locus } & \multirow[b]{2}{*}{ Allele } & \multicolumn{6}{|c|}{ Sample } \\
\hline & & D3 & D4 & G1 & G2 & K2 & K4 \\
\hline \multirow[t]{6}{*}{ Ano3 } & $\mathbf{N}$ & 21 & 30 & 35 & 22 & 20 & 25 \\
\hline & 162 & 0.500 & 0.500 & 0.500 & 0.500 & 0.500 & 0.500 \\
\hline & 164 & 0.000 & 0.000 & 0.000 & 0.000 & 0.000 & 0.000 \\
\hline & 174 & 0.000 & 0.000 & 0.000 & 0.000 & 0.000 & 0.000 \\
\hline & 176 & 0.500 & 0.483 & 0.500 & 0.500 & 0.500 & 0.480 \\
\hline & 178 & 0.000 & 0.017 & 0.000 & 0.000 & 0.000 & 0.020 \\
\hline \multirow[t]{8}{*}{ Ano7 } & $\mathbf{N}$ & 21 & 30 & 35 & 22 & 20 & 25 \\
\hline & 230 & 0.500 & 0.500 & 0.500 & 0.500 & 0.500 & 0.500 \\
\hline & 232 & 0.000 & 0.000 & 0.000 & 0.000 & 0.000 & 0.000 \\
\hline & 240 & 0.000 & 0.000 & 0.000 & 0.000 & 0.000 & 0.000 \\
\hline & 242 & 0.000 & 0.000 & 0.029 & 0.023 & 0.000 & 0.100 \\
\hline & 244 & 0.500 & 0.500 & 0.471 & 0.477 & 0.500 & 0.400 \\
\hline & 246 & 0.000 & 0.000 & 0.000 & 0.000 & 0.000 & 0.000 \\
\hline & 248 & 0.000 & 0.000 & 0.000 & 0.000 & 0.000 & 0.000 \\
\hline \multirow[t]{19}{*}{ Ano8 } & $\mathbf{N}$ & 21 & 30 & 35 & 22 & 20 & 25 \\
\hline & 208 & 0.000 & 0.000 & 0.000 & 0.000 & 0.025 & 0.000 \\
\hline & 210 & 0.000 & 0.000 & 0.114 & 0.182 & 0.000 & 0.040 \\
\hline & 212 & 0.048 & 0.233 & 0.314 & 0.273 & 0.325 & 0.260 \\
\hline & 214 & 0.143 & 0.100 & 0.071 & 0.045 & 0.150 & 0.180 \\
\hline & 216 & 0.143 & 0.167 & 0.000 & 0.000 & 0.000 & 0.020 \\
\hline & 218 & 0.167 & 0.000 & 0.000 & 0.000 & 0.000 & 0.000 \\
\hline & 254 & 0.000 & 0.000 & 0.000 & 0.000 & 0.000 & 0.000 \\
\hline & 256 & 0.000 & 0.017 & 0.000 & 0.000 & 0.000 & 0.000 \\
\hline & 264 & 0.000 & 0.000 & 0.271 & 0.023 & 0.000 & 0.020 \\
\hline & 266 & 0.000 & 0.000 & 0.143 & 0.273 & 0.050 & 0.000 \\
\hline & 268 & 0.000 & 0.000 & 0.057 & 0.159 & 0.000 & 0.060 \\
\hline & 270 & 0.000 & 0.000 & 0.000 & 0.045 & 0.050 & 0.000 \\
\hline & 272 & 0.095 & 0.100 & 0.029 & 0.000 & 0.100 & 0.060 \\
\hline & 274 & 0.119 & 0.217 & 0.000 & 0.000 & 0.225 & 0.280 \\
\hline & 276 & 0.286 & 0.133 & 0.000 & 0.000 & 0.050 & 0.080 \\
\hline & 278 & 0.000 & 0.033 & 0.000 & 0.000 & 0.025 & 0.000 \\
\hline & 282 & 0.000 & 0.000 & 0.000 & 0.000 & 0.000 & 0.000 \\
\hline & 284 & 0.000 & 0.000 & 0.000 & 0.000 & 0.000 & 0.000 \\
\hline
\end{tabular}


Supplementary Table 3.1 (c): Allele frequencies and sample sizes for Chapter 3, for loci with $>2$ alleles only (loci with two alleles had 0.500:0.500 allele frequencies). Samples K6 - P2.

\begin{tabular}{|c|c|c|c|c|c|c|c|}
\hline \multirow[b]{2}{*}{ Locus } & \multirow[b]{2}{*}{ Allele } & \multicolumn{6}{|c|}{ Sample } \\
\hline & & K6 & K7 & M1 & M2 & P1 & P2 \\
\hline \multirow[t]{6}{*}{ Ano3 } & $\mathbf{N}$ & 35 & 27 & 26 & 28 & 30 & 29 \\
\hline & 162 & 0.500 & 0.500 & 0.500 & 0.500 & 0.500 & 0.500 \\
\hline & 164 & 0.000 & 0.000 & 0.000 & 0.000 & 0.000 & 0.000 \\
\hline & 174 & 0.071 & 0.000 & 0.000 & 0.000 & 0.000 & 0.000 \\
\hline & 176 & 0.429 & 0.500 & 0.500 & 0.500 & 0.500 & 0.500 \\
\hline & 178 & 0.000 & 0.000 & 0.000 & 0.000 & 0.000 & 0.000 \\
\hline \multirow[t]{8}{*}{ Ano 7} & $\mathbf{N}$ & 35 & 27 & 26 & 28 & 30 & 29 \\
\hline & 230 & 0.500 & 0.500 & 0.500 & 0.500 & 0.500 & 0.500 \\
\hline & 232 & 0.000 & 0.000 & 0.000 & 0.000 & 0.000 & 0.000 \\
\hline & 240 & 0.000 & 0.000 & 0.000 & 0.000 & 0.000 & 0.000 \\
\hline & 242 & 0.300 & 0.037 & 0.000 & 0.000 & 0.017 & 0.017 \\
\hline & 244 & 0.200 & 0.463 & 0.308 & 0.393 & 0.483 & 0.483 \\
\hline & 246 & 0.000 & 0.000 & 0.154 & 0.071 & 0.000 & 0.000 \\
\hline & 248 & 0.000 & 0.000 & 0.038 & 0.036 & 0.000 & 0.000 \\
\hline \multirow[t]{19}{*}{ Ano8 } & $\mathbf{N}$ & 35 & 27 & 26 & 28 & 30 & 29 \\
\hline & 208 & 0.000 & 0.000 & 0.000 & 0.000 & 0.000 & 0.000 \\
\hline & 210 & 0.000 & 0.000 & 0.000 & 0.000 & 0.000 & 0.000 \\
\hline & 212 & 0.243 & 0.296 & 0.019 & 0.107 & 0.000 & 0.000 \\
\hline & 214 & 0.229 & 0.185 & 0.442 & 0.375 & 0.050 & 0.000 \\
\hline & 216 & 0.029 & 0.019 & 0.038 & 0.018 & 0.433 & 0.483 \\
\hline & 218 & 0.000 & 0.000 & 0.000 & 0.000 & 0.017 & 0.017 \\
\hline & 254 & 0.014 & 0.000 & 0.000 & 0.000 & 0.000 & 0.000 \\
\hline & 256 & 0.000 & 0.000 & 0.000 & 0.000 & 0.000 & 0.000 \\
\hline & 264 & 0.000 & 0.000 & 0.000 & 0.000 & 0.000 & 0.000 \\
\hline & 266 & 0.286 & 0.000 & 0.000 & 0.000 & 0.000 & 0.000 \\
\hline & 268 & 0.029 & 0.037 & 0.000 & 0.000 & 0.000 & 0.000 \\
\hline & 270 & 0.000 & 0.167 & 0.000 & 0.000 & 0.000 & 0.000 \\
\hline & 272 & 0.057 & 0.111 & 0.000 & 0.036 & 0.017 & 0.086 \\
\hline & 274 & 0.100 & 0.185 & 0.462 & 0.446 & 0.100 & 0.379 \\
\hline & 276 & 0.014 & 0.000 & 0.038 & 0.018 & 0.383 & 0.034 \\
\hline & 278 & 0.000 & 0.000 & 0.000 & 0.000 & 0.000 & 0.000 \\
\hline & 282 & 0.000 & 0.000 & 0.000 & 0.000 & 0.000 & 0.000 \\
\hline & 284 & 0.000 & 0.000 & 0.000 & 0.000 & 0.000 & 0.000 \\
\hline
\end{tabular}


Supplementary Table 4.1 (a): Allele frequencies and sample sizes for Chapter 4, for loci with $>2$ alleles only (loci with two alleles had 0.500:0.500 allele frequencies). Samples A1- B3.

\begin{tabular}{|c|c|c|c|c|c|c|}
\hline \multirow[b]{2}{*}{ Locus } & \multirow[b]{2}{*}{ Allele } & \multicolumn{5}{|c|}{ Sample } \\
\hline & & A1 & $\mathbf{A 2}$ & B1 & B2 & B3 \\
\hline \multirow[t]{6}{*}{ Ano3 } & $\mathbf{N}$ & 31 & 18 & 32 & 29 & 10 \\
\hline & 162 & 0.500 & 0.500 & 0.484 & 0.500 & 0.500 \\
\hline & 164 & 0.000 & 0.000 & 0.016 & 0.000 & 0.000 \\
\hline & 174 & 0.000 & 0.000 & 0.000 & 0.000 & 0.000 \\
\hline & 176 & 0.500 & 0.500 & 0.500 & 0.500 & 0.500 \\
\hline & 178 & 0.000 & 0.000 & 0.000 & 0.000 & 0.000 \\
\hline \multirow[t]{8}{*}{ Ano 7} & $\mathbf{N}$ & 31 & 18 & 32 & 29 & 10 \\
\hline & 230 & 0.500 & 0.500 & 0.500 & 0.483 & 0.500 \\
\hline & 232 & 0.000 & 0.000 & 0.000 & 0.017 & 0.000 \\
\hline & 240 & 0.000 & 0.000 & 0.000 & 0.000 & 0.000 \\
\hline & 242 & 0.000 & 0.000 & 0.016 & 0.103 & 0.000 \\
\hline & 244 & 0.500 & 0.500 & 0.453 & 0.362 & 0.500 \\
\hline & 246 & 0.000 & 0.000 & 0.031 & 0.034 & 0.000 \\
\hline & 248 & 0.000 & 0.000 & 0.000 & 0.000 & 0.000 \\
\hline \multirow[t]{18}{*}{ Ano8 } & $\mathbf{N}$ & 31 & 18 & 32 & 29 & 10 \\
\hline & 208 & 0.000 & 0.000 & 0.000 & 0.017 & 0.000 \\
\hline & 210 & 0.000 & 0.000 & 0.063 & 0.017 & 0.000 \\
\hline & 212 & 0.000 & 0.000 & 0.328 & 0.310 & 0.000 \\
\hline & 214 & 0.048 & 0.000 & 0.219 & 0.207 & 0.000 \\
\hline & 216 & 0.419 & 0.500 & 0.031 & 0.017 & 0.500 \\
\hline & 218 & 0.048 & 0.000 & 0.000 & 0.000 & 0.000 \\
\hline & 256 & 0.000 & 0.000 & 0.000 & 0.000 & 0.000 \\
\hline & 262 & 0.000 & 0.000 & 0.000 & 0.000 & 0.000 \\
\hline & 264 & 0.000 & 0.000 & 0.000 & 0.000 & 0.000 \\
\hline & 266 & 0.000 & 0.000 & 0.000 & 0.000 & 0.000 \\
\hline & 268 & 0.000 & 0.000 & 0.016 & 0.034 & 0.500 \\
\hline & 270 & 0.032 & 0.000 & 0.031 & 0.138 & 0.000 \\
\hline & 272 & 0.097 & 0.028 & 0.094 & 0.086 & 0.000 \\
\hline & 274 & 0.339 & 0.167 & 0.094 & 0.138 & 0.000 \\
\hline & 276 & 0.016 & 0.306 & 0.125 & 0.000 & 0.000 \\
\hline & 278 & 0.000 & 0.000 & 0.000 & 0.017 & 0.000 \\
\hline & 284 & 0.000 & 0.000 & 0.000 & 0.017 & 0.000 \\
\hline
\end{tabular}


Supplementary Table 4.1 (b): Allele frequencies and sample sizes for Chapter 4, for loci with $>2$ alleles only (loci with two alleles had 0.500:0.500 allele frequencies). Samples D2 - G1.

\begin{tabular}{|c|c|c|c|c|c|c|}
\hline \multirow[b]{2}{*}{ Locus } & \multirow[b]{2}{*}{ Allele } & \multicolumn{5}{|c|}{ Sample } \\
\hline & & D2 & D3 & D4 & D6 & G1 \\
\hline \multirow[t]{6}{*}{ Ano3 } & $\mathbf{N}$ & 24 & 27 & 25 & 20 & 35 \\
\hline & 162 & 0.500 & 0.500 & 0.500 & 0.500 & 0.500 \\
\hline & 164 & 0.000 & 0.000 & 0.000 & 0.000 & 0.000 \\
\hline & 174 & 0.000 & 0.000 & 0.000 & 0.050 & 0.000 \\
\hline & 176 & 0.250 & 0.500 & 0.500 & 0.450 & 0.500 \\
\hline & 178 & 0.250 & 0.000 & 0.000 & 0.000 & 0.000 \\
\hline \multirow[t]{8}{*}{ Ano 7} & $\mathbf{N}$ & 24 & 27 & 25 & 20 & 35 \\
\hline & 230 & 0.500 & 0.500 & 0.500 & 0.500 & 0.500 \\
\hline & 232 & 0.000 & 0.000 & 0.000 & 0.000 & 0.000 \\
\hline & 240 & 0.021 & 0.000 & 0.000 & 0.025 & 0.000 \\
\hline & 242 & 0.083 & 0.000 & 0.000 & 0.450 & 0.029 \\
\hline & 244 & 0.396 & 0.500 & 0.500 & 0.025 & 0.471 \\
\hline & 246 & 0.000 & 0.000 & 0.000 & 0.000 & 0.000 \\
\hline & 248 & 0.000 & 0.000 & 0.000 & 0.000 & 0.000 \\
\hline \multirow[t]{18}{*}{ Ano 8} & $\mathbf{N}$ & 24 & 27 & 25 & 20 & 35 \\
\hline & 208 & 0.000 & 0.000 & 0.000 & 0.000 & 0.000 \\
\hline & 210 & 0.000 & 0.000 & 0.000 & 0.000 & 0.114 \\
\hline & 212 & 0.000 & 0.130 & 0.220 & 0.000 & 0.314 \\
\hline & 214 & 0.000 & 0.148 & 0.120 & 0.025 & 0.071 \\
\hline & 216 & 0.500 & 0.278 & 0.200 & 0.450 & 0.000 \\
\hline & 218 & 0.000 & 0.130 & 0.000 & 0.025 & 0.000 \\
\hline & 256 & 0.000 & 0.000 & 0.020 & 0.000 & 0.000 \\
\hline & 262 & 0.000 & 0.000 & 0.000 & 0.000 & 0.000 \\
\hline & 264 & 0.000 & 0.000 & 0.000 & 0.000 & 0.271 \\
\hline & 266 & 0.000 & 0.000 & 0.000 & 0.000 & 0.143 \\
\hline & 268 & 0.000 & 0.000 & 0.000 & 0.000 & 0.057 \\
\hline & 270 & 0.000 & 0.000 & 0.000 & 0.000 & 0.000 \\
\hline & 272 & 0.000 & 0.056 & 0.060 & 0.000 & 0.029 \\
\hline & 274 & 0.458 & 0.074 & 0.200 & 0.450 & 0.000 \\
\hline & 276 & 0.042 & 0.185 & 0.140 & 0.050 & 0.000 \\
\hline & 278 & 0.000 & 0.000 & 0.040 & 0.000 & 0.000 \\
\hline & 284 & 0.000 & 0.000 & 0.000 & 0.000 & 0.000 \\
\hline
\end{tabular}


Supplementary Table 4.1 (c): Allele frequencies and sample sizes for Chapter 4, for loci with $>2$ alleles only (loci with two alleles had 0.500:0.500 allele frequencies). Samples G2 -K6.

\begin{tabular}{|c|c|c|c|c|c|c|}
\hline \multirow[b]{2}{*}{ Locus } & \multirow[b]{2}{*}{ Allele } & \multicolumn{5}{|c|}{ Sample } \\
\hline & & G2 & K1 & K4 & K5 & K6 \\
\hline \multirow[t]{6}{*}{ Ano3 } & $\mathbf{N}$ & 22 & 19 & 28 & 20 & 21 \\
\hline & 162 & 0.500 & 0.500 & 0.500 & 0.500 & 0.500 \\
\hline & 164 & 0.000 & 0.000 & 0.000 & 0.000 & 0.000 \\
\hline & 174 & 0.000 & 0.000 & 0.000 & 0.000 & 0.119 \\
\hline & 176 & 0.500 & 0.500 & 0.482 & 0.150 & 0.381 \\
\hline & 178 & 0.000 & 0.000 & 0.018 & 0.350 & 0.000 \\
\hline \multirow[t]{8}{*}{ Ano7 } & $\mathbf{N}$ & 22 & 19 & 28 & 20 & 21 \\
\hline & 230 & 0.500 & 0.500 & 0.500 & 0.500 & 0.500 \\
\hline & 232 & 0.000 & 0.000 & 0.000 & 0.000 & 0.000 \\
\hline & 240 & 0.000 & 0.000 & 0.000 & 0.000 & 0.000 \\
\hline & 242 & 0.000 & 0.474 & 0.089 & 0.500 & 0.500 \\
\hline & 244 & 0.500 & 0.000 & 0.411 & 0.000 & 0.000 \\
\hline & 246 & 0.000 & 0.026 & 0.000 & 0.000 & 0.000 \\
\hline & 248 & 0.000 & 0.000 & 0.000 & 0.000 & 0.000 \\
\hline \multirow[t]{18}{*}{ Ano8 } & $\mathbf{N}$ & 22 & 19 & 28 & 20 & 21 \\
\hline & 208 & 0.000 & 0.000 & 0.000 & 0.000 & 0.000 \\
\hline & 210 & 0.205 & 0.000 & 0.036 & 0.000 & 0.000 \\
\hline & 212 & 0.273 & 0.132 & 0.339 & 0.500 & 0.310 \\
\hline & 214 & 0.045 & 0.342 & 0.161 & 0.000 & 0.143 \\
\hline & 216 & 0.000 & 0.026 & 0.018 & 0.000 & 0.048 \\
\hline & 218 & 0.000 & 0.000 & 0.000 & 0.000 & 0.000 \\
\hline & 256 & 0.000 & 0.000 & 0.000 & 0.000 & 0.000 \\
\hline & 262 & 0.000 & 0.000 & 0.000 & 0.375 & 0.000 \\
\hline & 264 & 0.000 & 0.000 & 0.018 & 0.025 & 0.000 \\
\hline & 266 & 0.273 & 0.000 & 0.000 & 0.100 & 0.429 \\
\hline & 268 & 0.159 & 0.000 & 0.054 & 0.000 & 0.048 \\
\hline & 270 & 0.045 & 0.000 & 0.000 & 0.000 & 0.000 \\
\hline & 272 & 0.000 & 0.079 & 0.054 & 0.000 & 0.024 \\
\hline & 274 & 0.000 & 0.263 & 0.250 & 0.000 & 0.000 \\
\hline & 276 & 0.000 & 0.132 & 0.071 & 0.000 & 0.000 \\
\hline & 278 & 0.000 & 0.026 & 0.000 & 0.000 & 0.000 \\
\hline & 284 & 0.000 & 0.000 & 0.000 & 0.000 & 0.000 \\
\hline
\end{tabular}


Supplementary Table 4.1 (d): Allele frequencies and sample sizes for Chapter 4, for loci with $>2$ alleles only (loci with two alleles had 0.500:0.500 allele frequencies). Samples M2 - Y3.

\begin{tabular}{|c|c|c|c|c|c|c|}
\hline \multirow[b]{2}{*}{ Locus } & \multirow[b]{2}{*}{ Allele } & \multicolumn{5}{|c|}{ Sample } \\
\hline & & M2 & $\mathbf{P 2}$ & $\mathbf{P 1}$ & $\mathbf{T 1}$ & Y3 \\
\hline \multirow[t]{6}{*}{ Ano3 } & $\mathbf{N}$ & 25 & 30 & 19 & 10 & 15 \\
\hline & 162 & 0.500 & 0.500 & 0.500 & 0.500 & 0.500 \\
\hline & 164 & 0.000 & 0.000 & 0.000 & 0.000 & 0.000 \\
\hline & 174 & 0.000 & 0.000 & 0.000 & 0.000 & 0.033 \\
\hline & 176 & 0.500 & 0.500 & 0.500 & 0.500 & 0.467 \\
\hline & 178 & 0.000 & 0.000 & 0.000 & 0.000 & 0.000 \\
\hline \multirow[t]{8}{*}{ Ano 7} & $\mathbf{N}$ & 25 & 30 & 19 & 10 & 15 \\
\hline & 230 & 0.500 & 0.500 & 0.500 & 0.500 & 0.600 \\
\hline & 232 & 0.000 & 0.000 & 0.000 & 0.000 & 0.000 \\
\hline & 240 & 0.000 & 0.000 & 0.000 & 0.000 & 0.000 \\
\hline & 242 & 0.000 & 0.017 & 0.000 & 0.300 & 0.400 \\
\hline & 244 & 0.380 & 0.483 & 0.500 & 0.200 & 0.000 \\
\hline & 246 & 0.080 & 0.000 & 0.000 & 0.000 & 0.000 \\
\hline & 248 & 0.040 & 0.000 & 0.000 & 0.000 & 0.000 \\
\hline \multirow[t]{18}{*}{ Ano 8} & $\mathbf{N}$ & 25 & 30 & 19 & 10 & 15 \\
\hline & 208 & 0.000 & 0.000 & 0.000 & 0.000 & 0.000 \\
\hline & 210 & 0.000 & 0.000 & 0.000 & 0.000 & 0.000 \\
\hline & 212 & 0.120 & 0.000 & 0.000 & 0.050 & 0.000 \\
\hline & 214 & 0.380 & 0.000 & 0.079 & 0.150 & 0.233 \\
\hline & 216 & 0.000 & 0.500 & 0.447 & 0.300 & 0.267 \\
\hline & 218 & 0.000 & 0.017 & 0.026 & 0.000 & 0.000 \\
\hline & 256 & 0.000 & 0.000 & 0.000 & 0.000 & 0.000 \\
\hline & 262 & 0.000 & 0.000 & 0.000 & 0.000 & 0.000 \\
\hline & 264 & 0.000 & 0.000 & 0.000 & 0.000 & 0.000 \\
\hline & 266 & 0.000 & 0.000 & 0.000 & 0.000 & 0.000 \\
\hline & 268 & 0.000 & 0.000 & 0.000 & 0.450 & 0.000 \\
\hline & 270 & 0.000 & 0.000 & 0.000 & 0.000 & 0.100 \\
\hline & 272 & 0.000 & 0.083 & 0.026 & 0.050 & 0.167 \\
\hline & 274 & 0.480 & 0.367 & 0.000 & 0.000 & 0.233 \\
\hline & 276 & 0.020 & 0.033 & 0.421 & 0.000 & 0.000 \\
\hline & 278 & 0.000 & 0.000 & 0.000 & 0.000 & 0.000 \\
\hline & 284 & 0.000 & 0.000 & 0.000 & 0.000 & 0.000 \\
\hline
\end{tabular}




\section{Appendices}

\section{Computer code}

The taxon4blast.pl Perl script is publicly available at Github: https://github.com/AnnaFriedlander/taxon4blast

It is planned to tmake the SolexaStats java function available in a public repository in the future.

\section{Taxon4blast.pl README}
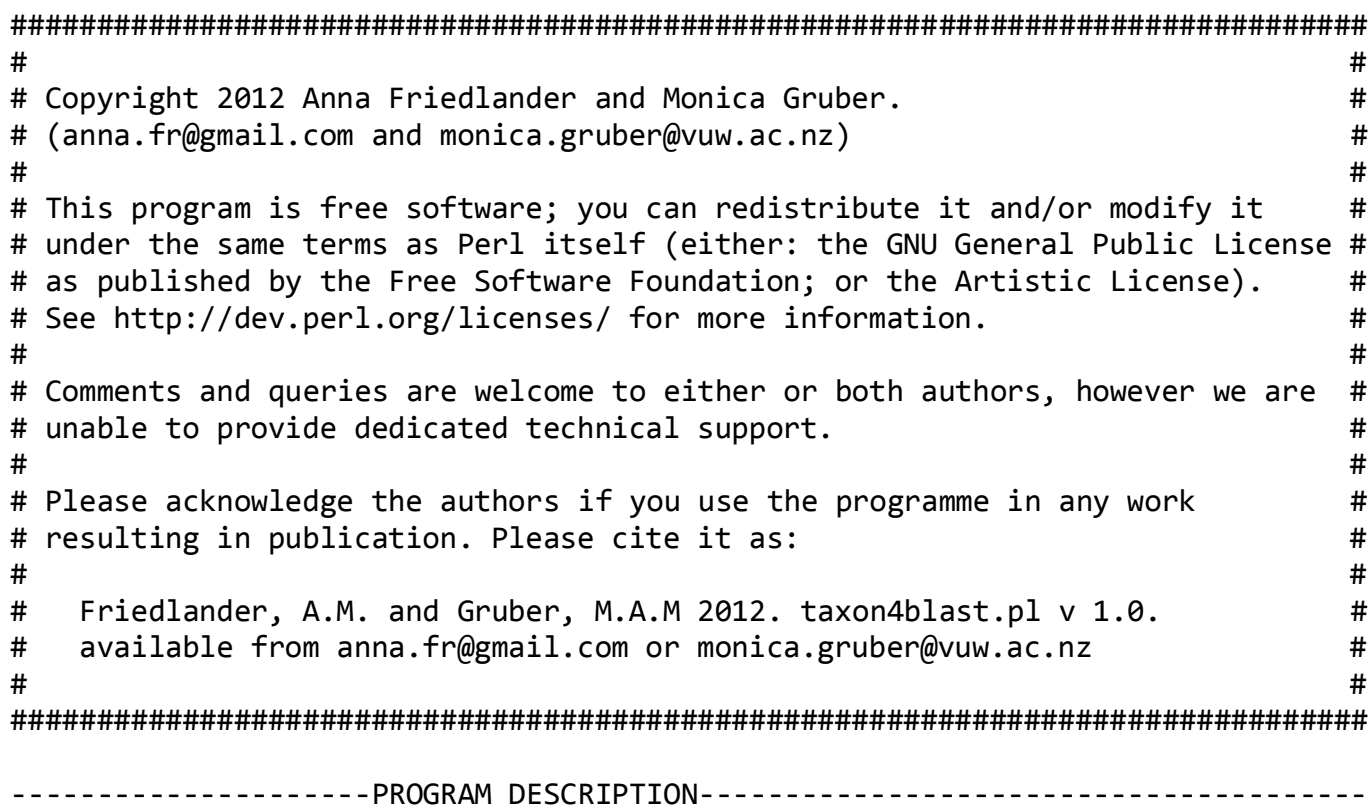

taxon4blast.pl is a program to parse BLAST+ output (output format 6 with default fields) and append taxonomic information.

It contains functions to:

* summarise BLAST+ output by taxon and calculate summary statistics (number of hits; total, average, and median sequence length; average e-value; average bit-score);

* compare BLAST+ output files by number of hits and sequence length at a particular taxon level (or taxon level and GI)

* create FASTA files and split data based on whether an input sequence hit a particular taxon.

More detailed information can be found in the help menu by using the command: $\%$ perl taxon4blast.pl -help

- RUNNING REQUIREMENTS

taxon4blast.pl parses files of the following format:

BLAST+ output in output format 6 (tab-delimited, no comment lines) with default fields (qseqid sseqid pident length mismatch gapopen qstart qend sstart send evalue bitscore)

To run taxon4blast.pl, Perl 5 (or later) and the following Perl modules must be installed:

*Bioperl 
*Bio: : LITE: :Taxonomy: :NCBI : :Gi2taxid

*Sort: : Naturally

As well as the default modules Getopt::Long and List::Util

The appropriate (nucl or prot) NCBI Taxonomy flatfiles are required:

*nodes.dmp

*names.dmp

*gi_taxid_nucl.dmp or gi_taxid_prot.dmp

which can be obtained from ftp://ftp.ncbi.nih.gov/pub/taxonomy (the first two files will be in a taxdump archive)

NOTE: the first time the program is run, use the gi_taxid_<nucl/prot>.dmp file; a gi_taxid_<nucl/prot>.bin file will be created, which can be used subsequent $\bar{l}$ y

To use -taxon_summary, input files to the BLAST+ search (in FASTA format) are required.

The following functions are available to the user:

taxon_info

fetches taxid and taxonomic hierarchy given GI, appends to BLAST+ output and prints to file (output used in the other four main functions)

taxon_summary

sorts taxon_info output according to taxon specified by the user, calculates summary stats (number of hits; total, average, median sequence length; average e-value; average bit-score) and prints to file

taxon_overlap

sorts taxon_info output according to taxon specified by user compares $\mathrm{n}$ files by number of hits and total length of hits for each taxon

taxon_sequence_overlap

sorts taxon info output according to taxon specified by user compares $\mathrm{n}$ files by number of hits and total length of hits for each taxon+GI

using -nonunique prints only records found in $>1$ file

sequence_extract

given taxon_info output and the original input files to BLAST (which were used to create the BLAST output used as input to taxon_info), and a taxon level and taxon name (e.g. -level superkingdom -name bacteria) this function creates two fasta files. One file contains all sequences that hit the taxon name specified, the other contains all sequences that hit other taxa. Note that there will likely be overlap in the output files (eg an input sequence may hit both bacterial sequences and nonbacterial sequences).

Using the subseq option prints out only the sequence that was matched (using qstart and qend)

-SUGGESTED IMPROVEMENTS -

The following are improvements that the user may wish to make to the program:

*allow input of any BLAST output type, by requiring the user to specify what column the qseqid, sseqid, evalue etc are in and how fields are delimited (at the moment BLAST+ output format 6 with default fields is hardcoded in).

* graphical output

*improve efficiency (e.g. use three hashes in taxon_info (line->gi; gi->taxid; taxid->taxon_info) - at present there is duplication in this function

*utilise parallel processing (at present processing is sequential) 
dataline_ $=$ (String) dataItr_.next () ;

for (int $i=0$; $i$ dataLine_.length(); $i++$ ) \{ numBasesConsidered_++; $c_{-}=$dataLine_. $\operatorname{charAt}(i)$; $\operatorname{switch}\left(c_{-}\right)\{$

$\begin{array}{lll}\text { //case ';': } & / /-5 & 0.7597 \\ \text { //case '<': } & / /-4 & 0.7153 \\ \text { //case '=': } & / /-3 & 0.6661\end{array}$

//case '=': $/ / /-3 \quad 0.6661$

//case '>': //-2 0.6131

//case '?': //-1 0.5573

//case '@': //0 0.5000

//case 'A': $/ / 100.4427$

//case 'B': $/ / 2 \quad 0.3869$

//case 'C': //3 0.3339

//case 'D': $/ / 4 \quad 0.2847$

//case 'E': $/ / 5 \quad 0.2403$

//case ' $F$ ': $/ / 6 \quad 0.2008$

//case 'G': $/ / 7 \quad 0.1663$

//case 'H': //8 0.1368

//case 'I': $/ / 9 \quad 0.1118$

//case 'J': $/ / 10 \quad 0.0909$

//case 'K': $/ / 11 \quad 0.0736$

//case 'L': $\quad / / 12 \quad 0.0594$

//case 'M': $/ / 13 \quad 0.0477$

//case ' $\mathrm{N}$ ': $/ / 140.0383$

//case '0': $/ / 15 \quad 0.0307$

//case ' $\mathrm{P}$ ': $/ / 16 \quad 0.0245$

//case 'Q': //17 0.0196

//case 'R': //18 0.0156

//case 'S': //19 0.0124

//case ' $T$ ': $/ / 20 \quad 0.0099$

//case 'U': //21 0.0079

//case 'V': $/ / 22 \quad 0.0063$

//case 'W': $/ / 23 \quad 0.0050$

//case ' $X$ ': $/ / 24 \quad 0.0040$

//case ' $Y$ ': $/ / 25 \quad 0.0032$

//case ' $Z$ ': $/ / 26 \quad 0.0025$

//case '[': $/ / 27 \quad 0.0020$

//case ' \/': //28 0.0016

//case ']': //29 0.0013

case '^': numBasesOverS30_++; break;//

case ' ' : numBasesOverS30_++; break;//

case ' $'$ : numBasesOver $530_{-}++$; break;//

case 'a': numBasesOverS30_++; break;//

case 'b': numBasesOverS30_++; break;//

case ' $c$ ': numBasesOverS30_++; break;//

case 'd': numBasesOverS30 ++ ; break;//

case 'e': numBasesOverS30_++; break;//

case 'f': numBasesOverS30_++; break;//

case 'g': numBasesOverS30_++; break;// \}

case ' $h$ ': numBasesOverS30_t+; break;//

$30 \quad 0.0010$

$31 \quad 0.0008$

$32 \quad 0.0006$

$33 \quad 0.0005$

$34 \quad 0.0004$

$35 \quad 0.0003$

$36 \quad 0.0003$

$37 \quad 0.0002$

$38 \quad 0.0002$

$39 \quad 0.0001$

\} \}

40

0.0001 


\section{Appendices}

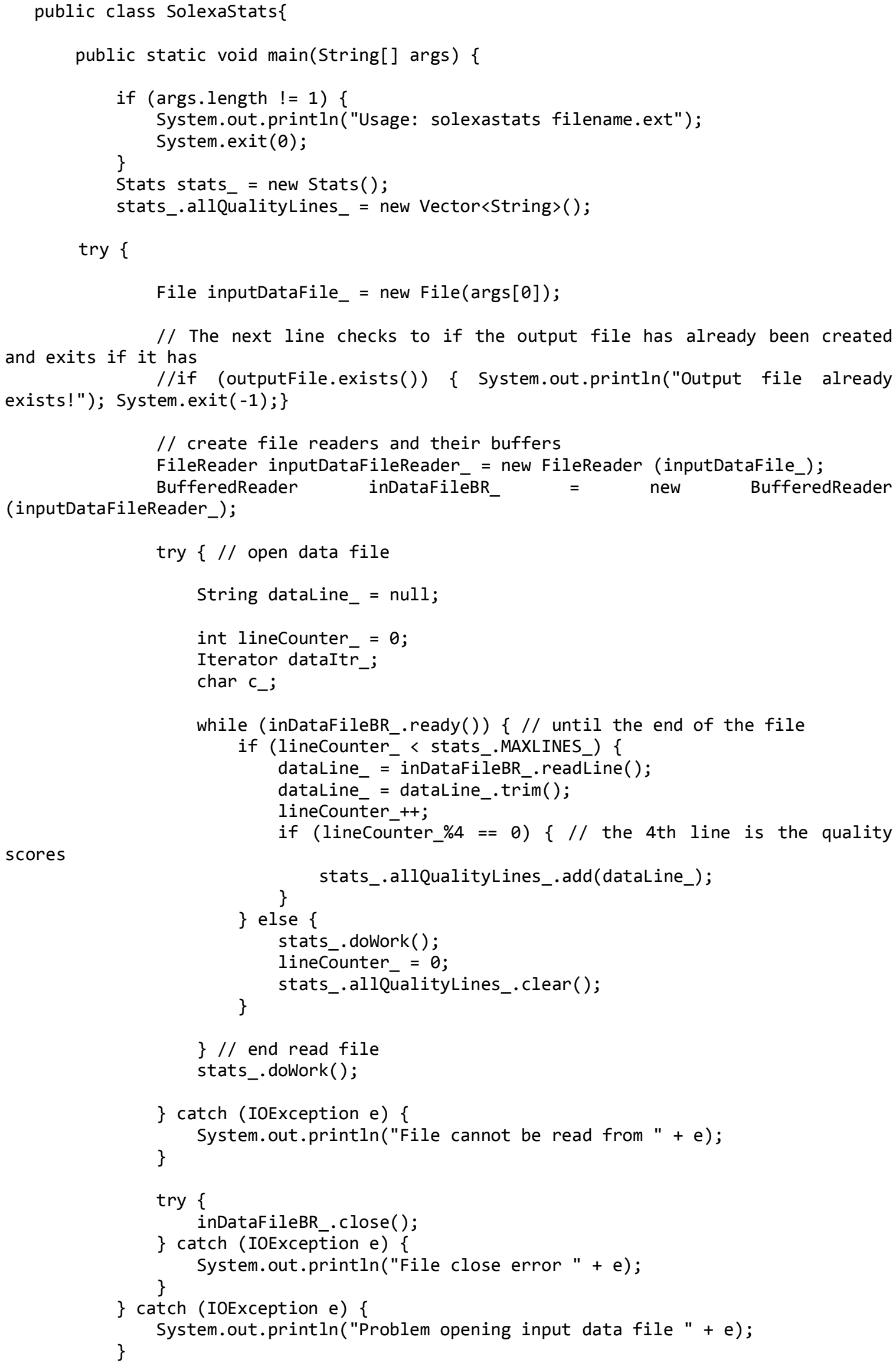


float megaBases_ $=$ (float) stats_.numBasesConsidered_ / 1000000;

float fractionPassed $=$ (float) stats.numBasesovers 30

stats_.numBasesConsidered_;

System.out.println(megaBases_ + " Megabases evaluated in " + args[0]);

System.out.println("The proportion of bases of Sanger quality score 30 or greater is "+ fractionPassed_);

\} \}

/* FASTQ symbol, ASCII number, Sanger score, $\mathrm{p}$ value

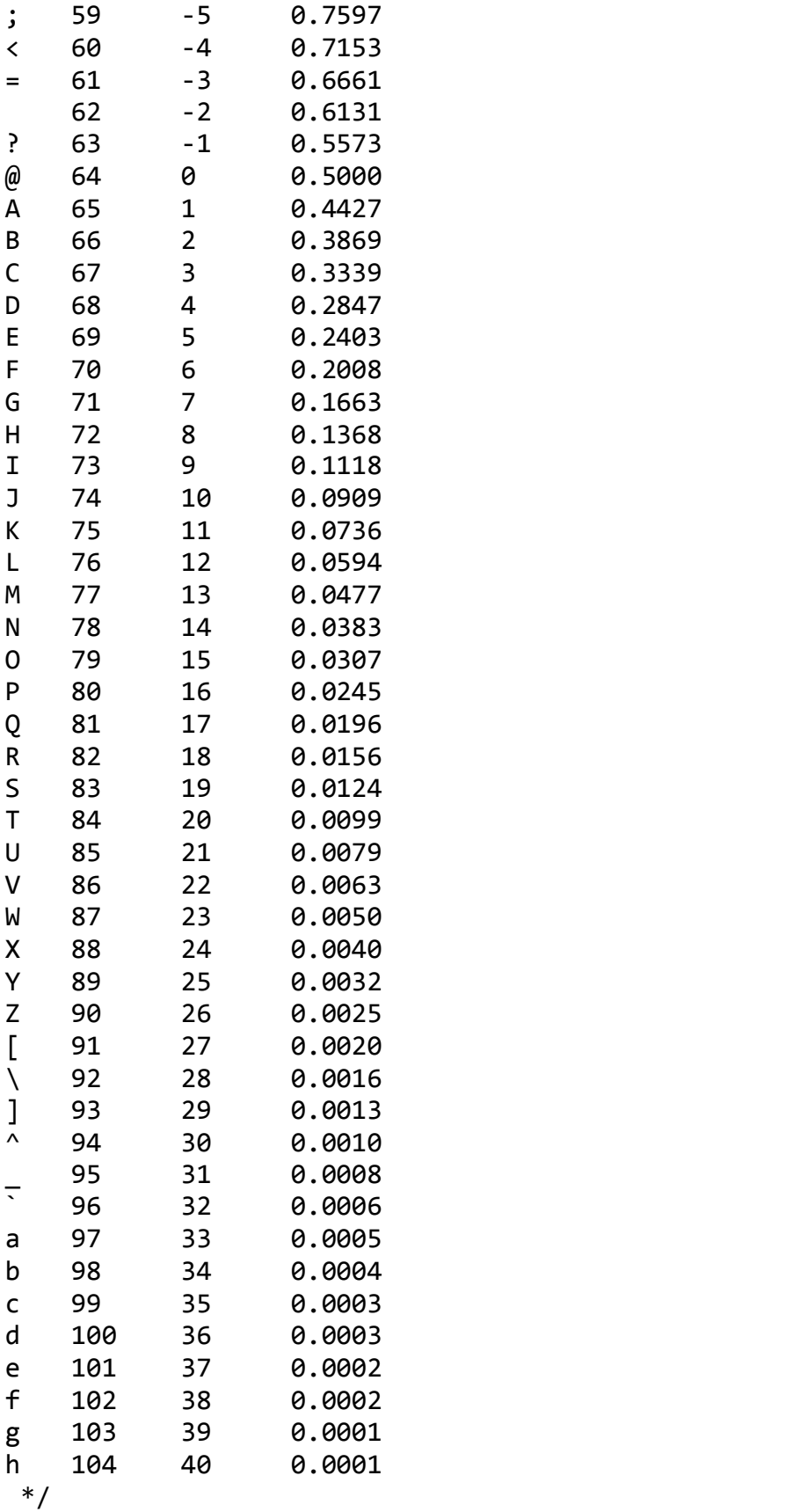

